SAND83 -7029

Unlimited Release

\title{
Dynamic-Stall Regulation of the Darrieus Turbine
}

J. W. Oler, J. H. Strickland

B. J. Im, G. H. Graham

Department of Mechanical Engineering

Texas Tech University

Lubbock, Texas 79409

Prepared by Sandia National Laboratories Albuquerque, New Mexico 87185 and Livermore, California 94550 for the United States Department of Energy under Conlract DE-AC04-76DP00789

Printed August 1983 


\section{DISCLAIMER}

This report was prepared as an account of work sponsored by an agency of the United States Government. Neither the United States Government nor any agency Thereof, nor any of their employees, makes any warranty, express or implied, or assumes any legal liability or responsibility for the accuracy, completeness, or usefulness of any information, apparatus, product, or process disclosed, or represents that its use would not infringe privately owned rights. Reference herein to any specific commercial product, process, or service by trade name, trademark, manufacturer, or otherwise does not necessarily constitute or imply its endorsement, recommendation, or favoring by the United States Government or any agency thereof. The views and opinions of authors expressed herein do not necessarily state or reflect those of the United States Government or any agency thereof. 


\section{DISCLAIMER}

Portions of this document may be illegible in electronic image products. Images are produced from the best available original document. 


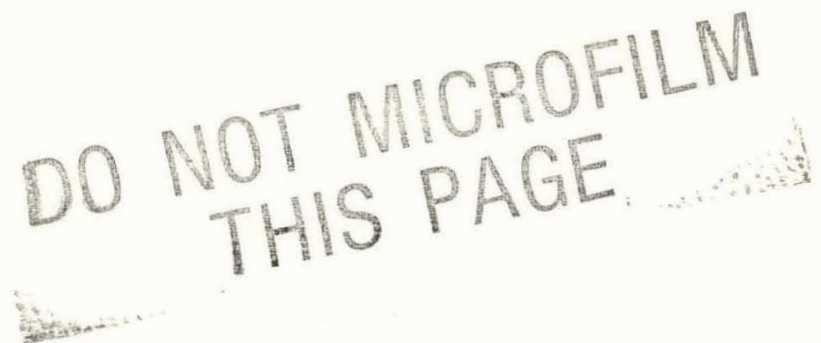

Issued by Sandia National Laboratories, operated for the United States Department of Energy by Sandia Corporation.

NOTICE: This report was prepared as an account of work sponsored by an agency of the United States Government. Neither the United States Government nor any agency thereof, nor any of their employees, nor any of their contractors, subcontractors, or their employees, makes any warranty, express or implied, or assumes any legal liability or responsibility for the accuracy, completeness, or usefulness of any information, apparatus, product, or procese dieclosed, or represents that its use would nnt infringe privately owned rights. Reference herein to any specific commercial product, process, or service by trade name, trademark, manufacturer, or otherwise, does not necessarily constitute or imply its endorsement, recommendation, or favoring by the United States Government, any agency thereof or any of their contractors or subcontractors. The views and opinions expressed herein do not necessarily state or reflect those of the United States Government, any agency thereof or any of their contractors or subcontractors.

Printed in the United States of America Available from

National Technical Information Service

U.S. Department of Commerce

5285 Port Royal Road

Springfield, VA 22161

NTIS price codes

Printed copy: A08

Microfiche copy: AU1 
SAND83-7029

Unlimited Release

Printed August 1983

\title{
Dynamic-Stall Regulation of the Darrieus Turbine
}

\author{
J. W. Oler, J. H. Strickland \\ B. J. Im, G. H. Graham \\ Department of Mechanical Engineering \\ Texas Tech University \\ Lubbock, Texas 79409 \\ Under Sandia Contract No. 74-1218
}

\begin{abstract}
A two-dimensional unsteady airfoil analysis is described which utilizes a doublet panel method to model the airfoil surface, an integral boundary layer scheme to model the viscous attached flow, and discrete vortices to model the detached boundary layers which form the airfoil wake region. This model has successfully predicted steady lift and drag coefficients as well as pressure distributions for several airfoils with both attached and detached boundary layers. Unsteady calculations have thus far been limited to attached flow situations. Instantaneous pressure distributions have also been obtained on a single-bladed rotor operating in a tow tank in order to provide experimental data for eventual comparison with analytical predictions.
\end{abstract}

\section{DISCLAIMER}

This report was prepared as an account of work sponsored by an agency of the United States Government. Neither the United States Government nor any agency thereof, nor any of their employees, makes any warranty, express or implied, or assumes any legal liability or responsibility for the accuracy, completeness, or usefulness of any information, apparatus, product, or process disclosed, or represents that its use would not infringe privately owned rights. Reference herein to any specific sommercial product, process, or service hy trade name, trademark, manufacturer, or otherwise does not necessarily constitute or imply its endorsement, recommendation, or favoring by the United States Government or any agency thereof. The views and opinions of authors expressed herein do not necessarily state or reflect those of the United States Government or any agency thereof.
NOTICE

PORTIONS OF THIS REPORT ARE ILLEGIBLE

it has been reproduced from the bes available copy to permit the broades possible availability.

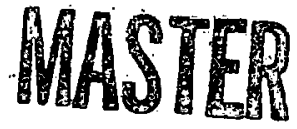

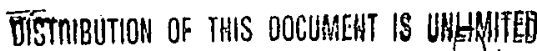


TABLE OF CONTENTS

1. INTRODUCTION $\ldots \ldots \ldots \ldots \ldots \ldots \ldots \ldots \ldots \ldots \ldots \ldots \ldots \ldots \ldots \ldots \ldots \ldots$

1.1 Research Motivation and objectives................... 1

1.2 Relationship of Research to Previous Work.............. 3

2. FORMULATION OF THE DYNAMIC STALL MODEL $\ldots \ldots \ldots \ldots \ldots \ldots \ldots \ldots \ldots \ldots$

2.1 Overview of the Complete Model.................... 7

2.2 The Potential Flow Mode1......................... 8

2.2.1 Mathematical Representation................... 8

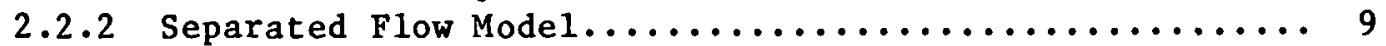

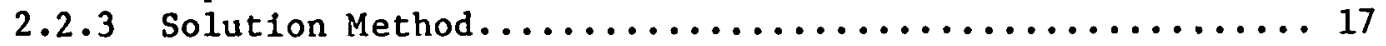

2.2 .4 Problem in Body Fixed Coordinates................. 20

2.2.5 Numerical Solution by the Collocation Method.......... 26

2.2.6 Evaluation of the Influence Coefficients........... 31

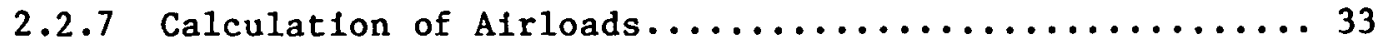

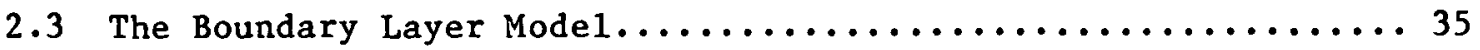

2.3 .1 Overview of Unsteady Boundary Layer Codes.......... 35

2.3 .2 Present Boundary Layer Mode1.................. 37

2.3 .3 Transition and Separation.................... 42

2.3.4 Numerical Solution of Boundary Layer Equations........ 44

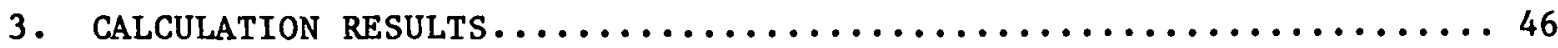

3.1 Steady, Attached Flows............................. 46

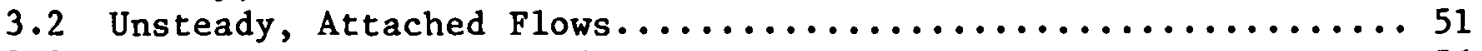

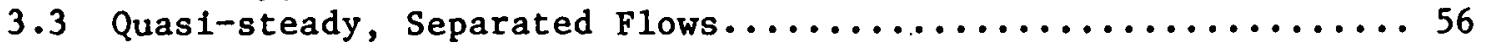

4. DYNAMIC STALL EXPERIMENT......................... 67

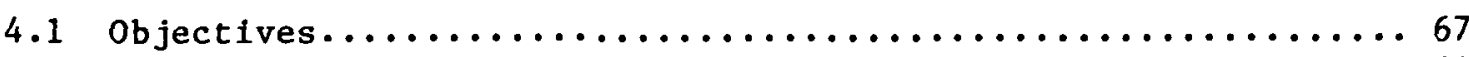

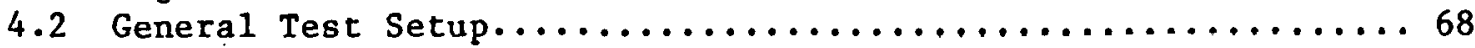

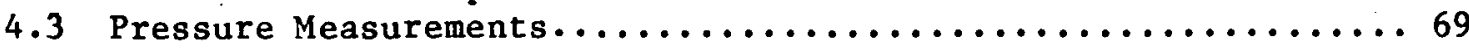

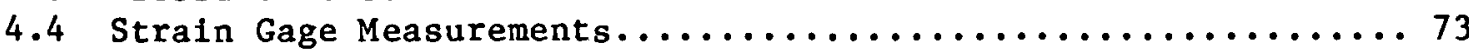

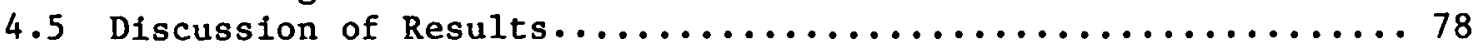

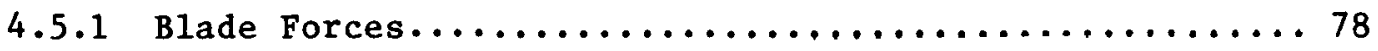

4.5 .2 Pressure Data............................. 87

5. CONCLUSIONS AND RECOMMENDATIONS $\ldots \ldots \ldots \ldots \ldots \ldots \ldots \ldots \ldots \ldots \ldots$

5.1 Summary of the Analyt1cal Study................... 97

5.2 Summary of the Experimental Investigation.............. 98

6. REFERENCES....................................... 99

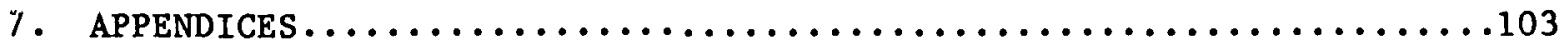

A. Fundamentals of Potential Aerodynamics.................. 104

B. An Integral Solution to LaPlace's Equation................116

C. Uniqueness Requirements for the Integral Solution...........122

D. Equivalent Doublet and Vortex Surface Distributions..........125

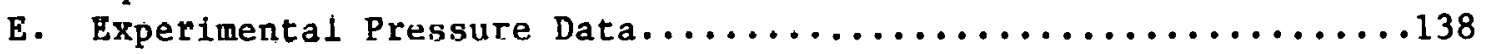




\section{INTRODUCTION}

\subsection{Research Motivation and Objectives}

The power produced by a Darrieus turbine at its regulation windspeed is much higher than would be anticlpated from an analysis based on steady airfoll data. The additional power output is a direct consequence of an unsteady flow phenomena known as dynamic stall. Above the regulation windspeed, the power output typically drops off abruptly.

Although the windspeed range over which the peak power is attained is relatively small, the turbine drive train and electrical power generation equipment must be sized to safely accept that maximum level of power output. Economic studies by Kadlec (1980) and Klimas (1980) have indicated that a 5 to 10 percent reduction in the cost of energy from a one megawatt Darrieus turbine could be achleved if the peak power output is reduced as shown in Figure 1.1. The actual loss of useable power is minimal due to the relatively infrequent occurrence of winds near the regulation windspeed. However, the reduced maximum power output allows the use of smaller and less expensive drive trains and generators. In addition, the overall efficiency of the wind energy conversion system is increased by the Improved matching of the aerodynamic and mechanical components.

To control the peak power output of a Darrieus turbine without adversely affecting its performance at low and medium windspeeds, it is necessary to tallor the dynamic stall characteristics of its blades. It is anticipated that "stall regulation" may be achieved through the design of 


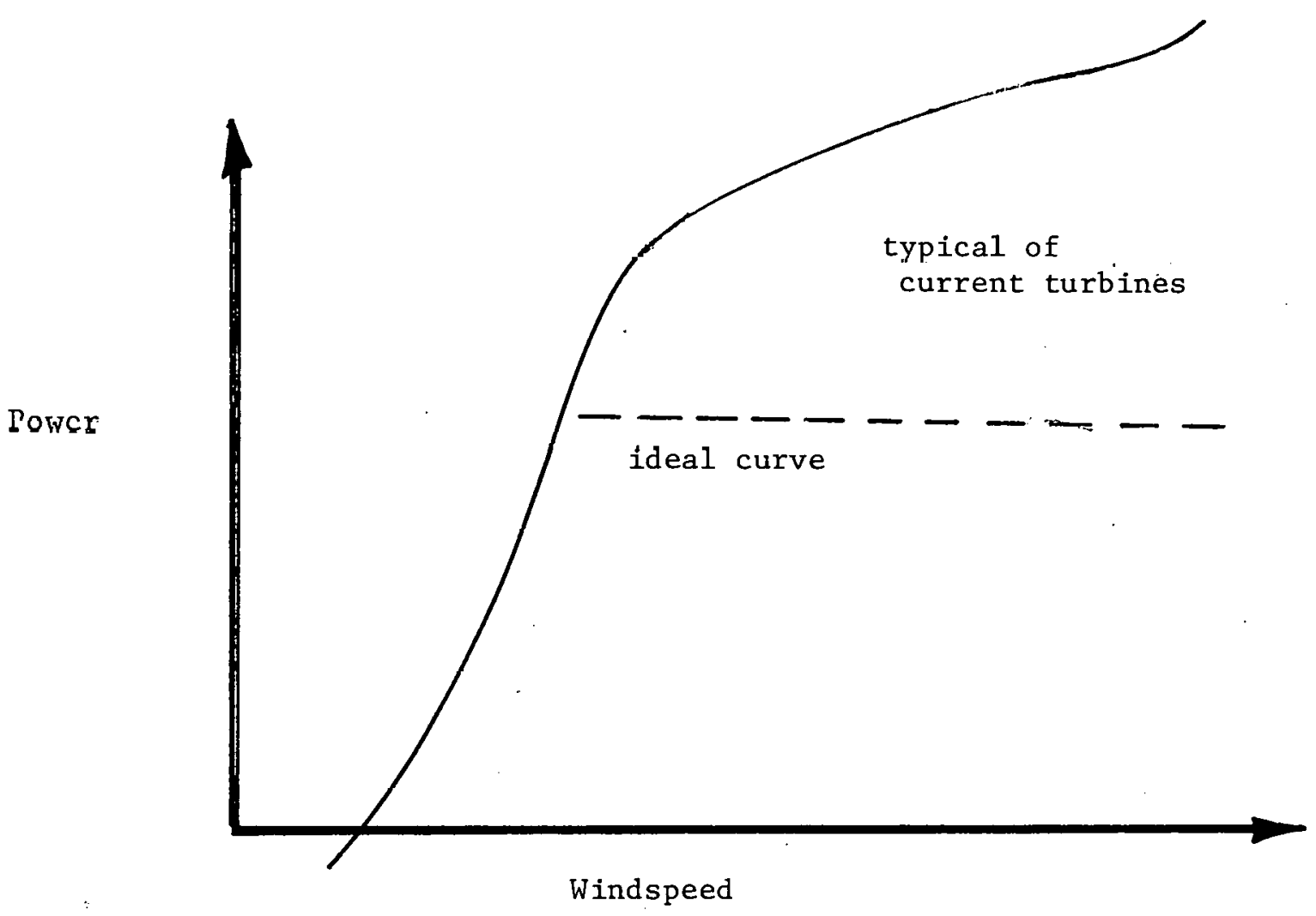

Figure 1.1 Power vs. Windspeed 
airfoll sections which elther passively exhibit the desired characteristics or have provision for active boundary layer control.

To facllitate the design and evaluation of the new airfoll sections, a valuable tool would be a numerical model capable of predicting the afrloads on an airfoil experfencing dynamic stall. The model would allow the examination of many more potential geometries than would be economically feastble if wind tunnel or full-scale turbine tests were required. The research reported herein has been concerned with the development of a numerical model having the capability to perform this function. The current work has been limited to the prediction of the unsteady separated flow over an airfoll at a constant angle of attack. However, as formulated, the model is directly applicable to unsteady airfoil motions as we11. Its extension to these cases is currently underway at Texas Tech University.

In addition to the theoretical work reported herein, an experimental program has been conducted. The purpose of this work was twofold. First, it has provided new insights into the mechanisms of dynamic stall and the way in which it influences Darrieus turbine performance. Secondly, it has generated new data which will eventually be utilized to test the predictive capabilities of the numerical model.

\subsection{Relationship of Research to Previous Work}

Airfoil models used in previous Darrieus turbine aerodynamic simulations can be classified as elther thin airfoll potential flow models or lifting line models with tabulated airfoll data. The airfoil model which is currently being formulated includes both viscous and unsteady effects and will be referred to as DYNA2 (DYNamic Airfoll model in $\underline{2}$ dimensions). 
The thin airfoil models which have been used by Wilson (1978) and Fanucci (1976) yield good results when the airfoil angles of attack are below stall thresholds and when the airfoll section being modeled is indeed thin. Empirical drag data are required to actually compute the alrfoil thrust coefficient. The thin airfoll models do include the important dynamic effects due to pitching and certain added mass effects. They are totally inadequate for predicting static or dynamic stal.1.

The lifting line model which has beul used by Gtrickland (1976) yields good results when the flow can be considered as quasi steady. Dynamic effects can be estimated using empirical relationships as was recently demonstrated by Klimas (1980) who used the Boeing-Vertol Mode1 by Gormont (1973). The lifting line model used in this fashion required that static Ifft and drag data for the airfoll in question be available.

The DYNA2 mode1 integrates analytical models for three separate regions of the flow. These three regions require utilization of panel methods to model the potential flow, discrete vortex wake methuds to model the separated shear flows, and the boundary layer methods to model the attached shear flows. Each of these analysis methods must include unsteady effects. A historical review of panel methods by Kraus (1978) reveals that one of the first uses of this type of method was by A.M.0. Smith (1962) for a body with zero lift. These methods have progressed to a point such that three-dimensional Iffting geometrles can be considered in buth subsonfe and supersonic flows as typified by the work of Woodward (1973). Many of the investigations using panel methods have, been for steady flows, although the general method is well suited to unsteady flows as is evidenced by the works of Ashley (1966), Djojodihardjo and Widnal1 (1969), Summa (1976), and oler 
(1976). Utilization of panel methods has been concentrated on attached nonseparating flows where the wake vorticity is shed smoothly from the trafling edge and the Kutta condition is satisfied. One recent exception is the utilization by NASA Langley of panel methods to examine the flow around a delta wing with leading edge separation.

The modeling of separated wakes using discrete vortices can be viewed as being a natural extension of unsteady panel methods. For instance, in the case of a stalling airfoll, the wake will consist of two wake surfaces instead of the usual one for unseparated flow. Clements (1975) gives a comprehensive review of wake modeling using discrete vortices. Many of the investigations reported by clements pertain to flow behind bluff bodies such as that due to Sarpkaya (1979) for flow behind circular cylinders. These bluff bodies were in all cases tmmersed in a fluid with steady, uniform freestream velocities. Most of the workers used potential flow models based on conformal mapping techniques as opposed to utilization of the more flexible panel methods. One example of wake modeling using discrete vorticcs is that due to Ham (1968) in which he modeled dynamic stall by allowing a single vortex to be shed from the leading edge of the airfoll at oome assumed angle of attack.

A number of unsteady turbulent boundary layer analyses are available in the 1iterature ranging from integral forms (e.g., Daneshyar, 1978) to oneequation closure models (e.g., Nash, et al., 1975), to multiple equation closure models. Due to the large number of boundary layer calculations required for unsteady cases, the simpler models should be used when. possible. Part of the work leading to the development of DYNA2 has been to extend the relatively successful integral method due to Head (1969) to Include unsteady effects. 
The major contribution of the DYNA2 mode1 and its possible extensions is that it provides a synthesis of existing techniques to provide a reasonably general unsteady afrfoll model which includes the prediction of dynamic stall. In reading the literature, it is apparent that much of the work has been compartmentalized into potential flow via panel methods, separated wakes via discrete vortices, and turbulent boundary layers via a number of closure models. Due to the relatively mature nature of the work in each of these areas, it therefore seems appropriate and timely to combine these techniques into a more complete model. 
2. FORMULATION OF THE DYNAMIC STALL MODEL

\subsection{Overview of the Complete Model}

There are two principal components contalned in DYNA2. These are the potential flow and boundary layer calculation routines. Both routines include unsteady effects and are coupled through the pressure distribution and boundary layer separation effects.

The potential flow calculations are accomplished with a finite element method which allows representation of the airfoll and wake surfaces by uniform strength doublet panels. The wake includes a surface extending from the boundary layer separation point as well as from the trailing edge. The routine predicts the position and strength of the wake surfaces and the corresponding pressure distribution and Integrated load on the afrfoil.

In the model's present form, the location of the boundary layer separation point is predicted on the basis of a pressure distribution for a steady, nonseparated flow. That location is considered fixed as the potential flow calculations proceed in a step-by-step manner. Obviously, a more correct arrangement would be to recalculate the separation point location at each time step. However, the difficulties (discussed in the next chapter) which have been encountered when that method was utilized have forced the temporary adoption of the present scheme. The resolution of the step-by-step boundary layer calculation problems is the subject of work currently underway at Tiexas Tech Universtty • 


\subsection{The Potential Flow Mode1}

\subsubsection{Mathematical Representation}

Consider the motion of a homogeneous, incompressible and inviscid fluid through which a body with its associated trailing wake moves. The body surface is given with respect to a fluid fixed reference frame by

$$
S(\vec{r}, t)=0
$$

and the trailing wake by

$$
\vec{w}(\vec{r}, \tau)=0
$$

The possibility of a separated flow is accounted for by allowing the wake to include surfaces of potential discontinulty emanating from a boundary layer separation point as well as from the trailing edge.

Noting that the body plus wake comprises a complete lifting system and assuming that the motion was started from a state of rest or uniform motion, it follows that the motion is irrotational for all times. The governing equation for the disturbance potential is given by

$$
\nabla_{\phi}^{2}(\vec{r}, t)=0
$$

Once the solution is known, the pressure discrlbution lin the flow may be found from the unsteady Bernoulli equation:

$$
P=P_{w}-\rho\left(\frac{\partial \phi}{\partial l}+\frac{1}{2} \nabla \phi^{2}\right)
$$

The determination of a unique solution of Eqn. 2.2.3 is accomplished through the application of the following boundary conditions:

1) The Infinity Condition -

The disturbance potential resulting from the presence of the body must vanish at infinity. 
2) The Kinematic Surface Tangency Condition -

On the surface, $S$, the normal relative flutd velocity must he ziero.

3) The Kutta Condition -

At all times, the flow of fluid from the trailing edge must be smooth and continuous.

4) The Boundary Layer Separation Condition -

The sheet of potential discontinuity from the boundary layer separation point must reflect the injection of the boundary layer vorticity.

5) The Dynamic Free Surface Condition -

The pressure must be continuous through the wake surfaces, since they cannot sustain a load.

6) The Geometric Free Surface Condition -

The wake particles are convected downstream at the local convection velocities.

\subsubsection{Ecparated Flow Model}

For the purpose of modeling, it is assumed that the wake may be adequately represented by two sheets of potential discontinuity. One surface extends from the trailing edge while the other originates at the boundary layer separation point as 1llustrated in Fig. 2.2.1.

The rates at which vorticity is shed into the two wake surfaces are related to the rate of change of the vorticity bound to the alrfoil surface by the Kelvin-Helmholtz vorticity conservation theorem. 


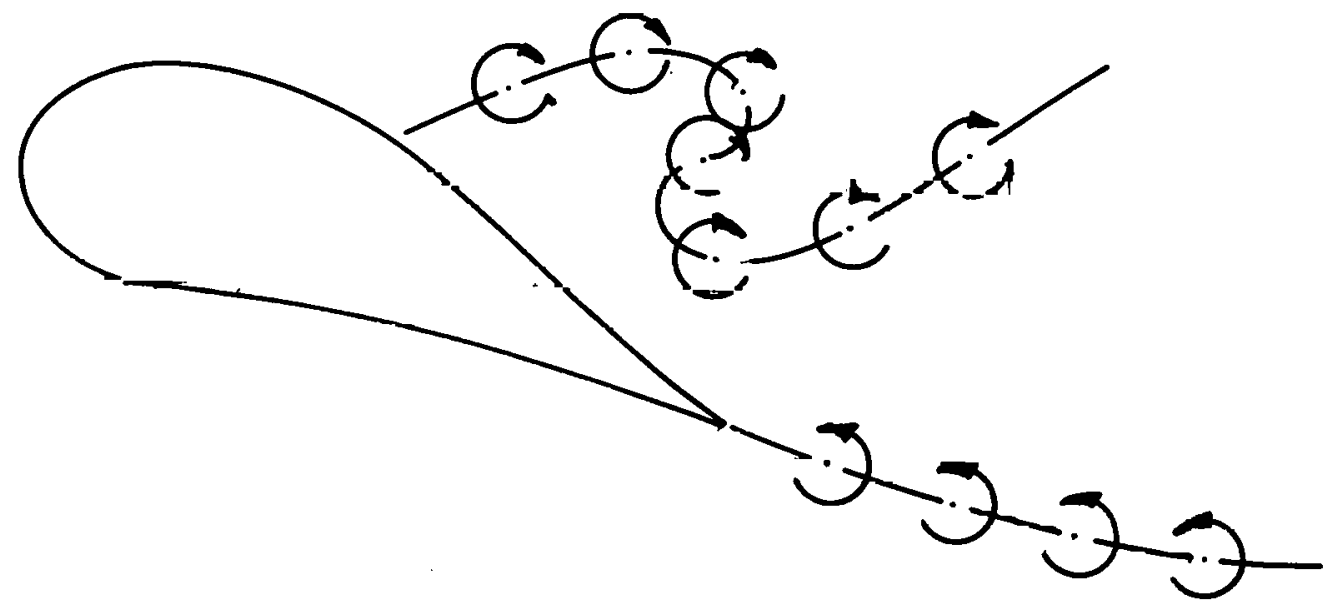

Figure 2.2.1 Separated Flow Model 
The theorem requires that the rate of change of net vorticity in the flow field is zero, i.e.,

$$
\frac{d \Gamma_{\text {net }}}{d t}=0
$$

or

$$
\frac{d \Gamma b}{d t}+\frac{d \Gamma w}{d t}+\frac{d \Gamma s}{d t}=0 .
$$

Here, the net vorticity has been divided into three components: the vorticity bound to the airfoll surface $\Gamma_{b}$, the vorticity shed from the boundary layer separation point $\Gamma_{s}$, and the vorticity shed from the trailing edge $\Gamma_{W}$. The time derivatives of $\Gamma_{W}$ and $\Gamma_{S}$ represent the rate of vorticity shedding to the respective wake surfaces.

A simple vorticity flux analysis may be utilized to estimate the vorticity shedding rate from the boundary layer separation point.

$$
\begin{aligned}
& \frac{d \Gamma s}{d t}=\int_{0}^{\delta} u\left(\frac{\partial u}{\partial y}-\frac{\partial v}{\partial x}\right) d y \\
& \frac{d \Gamma s}{d t} \cong \int_{0}^{\delta} \frac{1}{2} \frac{\partial u^{2}}{\partial y} d y \\
& \frac{d \Gamma s}{d t} \cong \frac{e^{2}}{2}
\end{aligned}
$$

where $U_{e}$ is the edge velocity. An assumption has been made here that $100 \%$ of the vorticity contained in the boundary layer is injected into the inviscid flow field at the separation point. This is actually an overestimate so that a reduction factor is needed in the actual ealculations. 
In Appendix D, it is demonstrated that the vortex sheet strength distribution is equal to the gradient of the potential jump across that sheet. If $\gamma_{b}$ is the vorticity per unit length along an airfoll surface and $\sigma$ is the distribution of potential discontinuity or doublet strength along the surface, then referring to Fig. 2.2.2, the bound vorticity may be written as

$$
\begin{aligned}
& \Gamma_{b}=\int_{A}^{B} \gamma_{b} d s \\
& \Gamma_{b}=\int_{A}^{B} \frac{d \sigma}{d s} d s \\
& \Gamma_{b}=\Delta \sigma_{T E} .
\end{aligned}
$$

The rate of change of bound vorticlty may be expressed in terms of the difference in surface doublet strength at the trailing edge:

$$
\frac{\mathrm{d} \Gamma}{\mathrm{dt}}=\frac{\mathrm{d}}{\mathrm{dt}}\left(\Delta \sigma_{\mathrm{TE}}\right)
$$

Suhstituting Eqns. 2.2 .6 and 2.2 .8 into Eqn. 2.2.5 provides an expression for the rate of shedding of vorticity to the wake surface extending from the tralling edge.

$$
\frac{\mathrm{d} \Gamma}{\mathrm{dt}}=-\left(\frac{\mathrm{d}}{\mathrm{dt}}\left(\Delta \sigma_{\mathrm{TE}}\right)+\frac{\mathrm{U}_{\mathrm{e}}^{2}}{2}\right)
$$

Recall that Eqn. 2.2 .9 was based upon the Kelvin-llelmholtz vorticity conservation theorem. The same result may be arrived at by applying the dynamic free surface boundary condition at the airfoil trailing edge. Specifically, the pressure must be continuous across the wake over its entire length, including the point of attachment at the trafling edge. There, the pressure difference across the infinitely thin surface is zero which leads to 


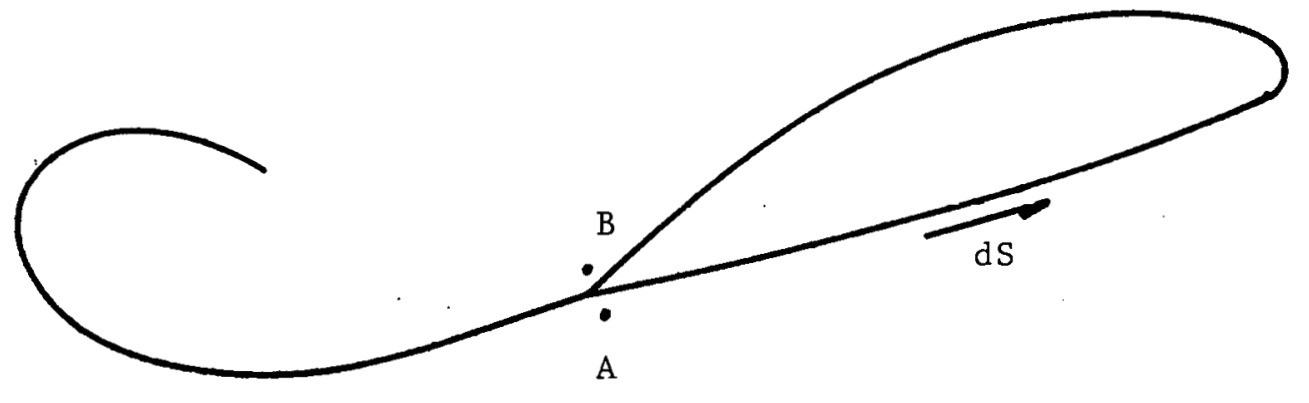

Figure 2.2.2 The Bound Vorticity on an Airfoil 


$$
\begin{aligned}
& 0=P_{u}-P_{\ell} \\
& 0=\frac{\partial}{\partial t}\left(\phi_{u}-\phi_{\ell}\right)+\frac{1}{2}\left(\nabla \phi_{u}^{2}-\nabla \phi_{l}^{2}\right) .
\end{aligned}
$$

Recognizing that $\nabla \phi=\vec{u}$ for the fluid fixed reference frame, then

$$
\frac{1}{2}\left(u_{u}^{2}-u_{\ell}^{2}\right)=-\frac{\partial}{\partial t}\left(\Delta \psi_{T E}\right)
$$

From Fig. 2.2.3, it is noted that $\left(U_{u}^{2}-U_{\ell}^{2}\right) / 2$ is the net rate of vorticity shedding from the boundary layers on the upper and lower surfaces of the airfoil at the ralling edge. Then,

$$
\frac{\mathrm{d} \Gamma_{\mathrm{w}}}{\mathrm{dt}}=-\frac{\mathrm{d}}{\mathrm{dt}}\left(\Delta \phi_{\mathrm{TE}}\right)
$$

By calculating the circulation about a curve, as shown in $\vec{F}$ ig. 2.2.4, which encloses the airfoil and wake surface extending from the boundary layer separation point, it is apparent that

$$
\frac{\mathrm{d}}{\mathrm{dt}}\left(\Delta \phi_{\mathrm{TE}}\right)=\frac{\mathrm{d} \Gamma_{\mathrm{b}}}{\mathrm{di}}+\frac{\mathrm{d} \Gamma_{\mathrm{s}}}{\mathrm{dt}}
$$

So;

$$
\frac{\mathrm{d} \Gamma \mathrm{w}}{\mathrm{dt}}=-\left(\frac{\mathrm{d} \Gamma \mathrm{b}}{\mathrm{dt}}+\frac{\mathrm{d} \Gamma \mathrm{s}}{\mathrm{dt}}\right)
$$

which is equivalent to the result obtained from the Kelvin-Helmholtz theoren.

An important consequence of boundary layer separation may be noted by applying the dynamic free surface condition to the wake surface extending from the separation point. Let points $A$ and $B$ be located an Infinftesimal distance ahead of and behind the boundary layer separation point. The pressure difference across the two poliuls must be zero which results in 


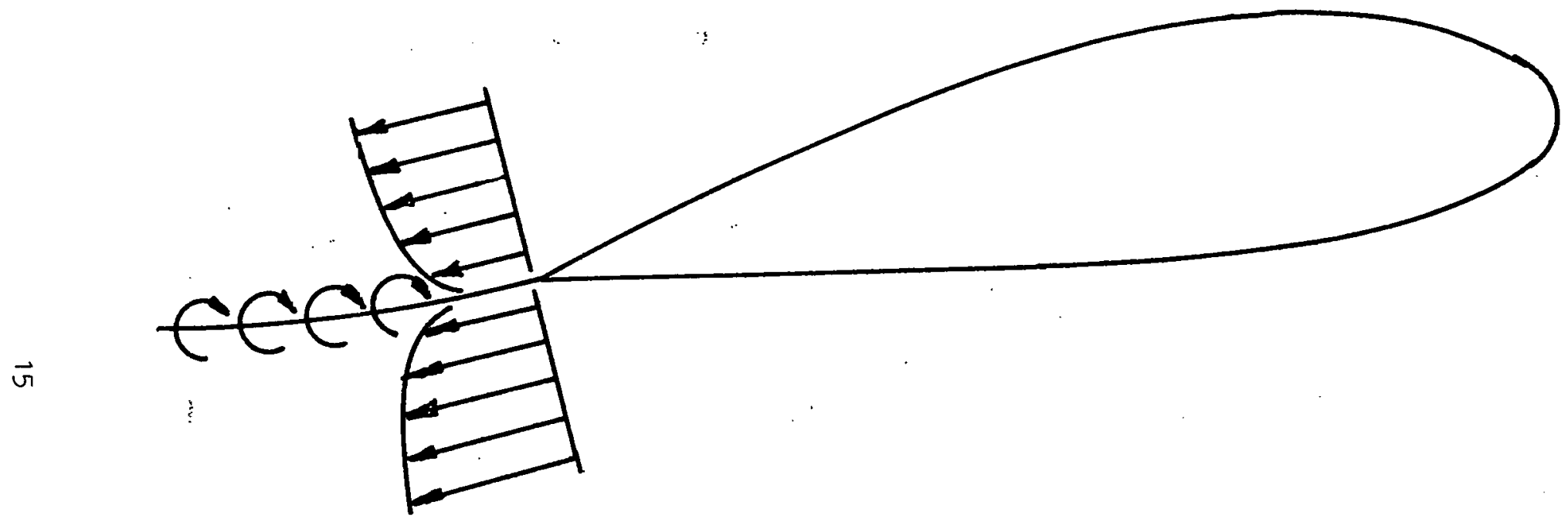

Figure 2.2.3 The Net Rate cf Vorticity Shedding at the Trailing Edge 


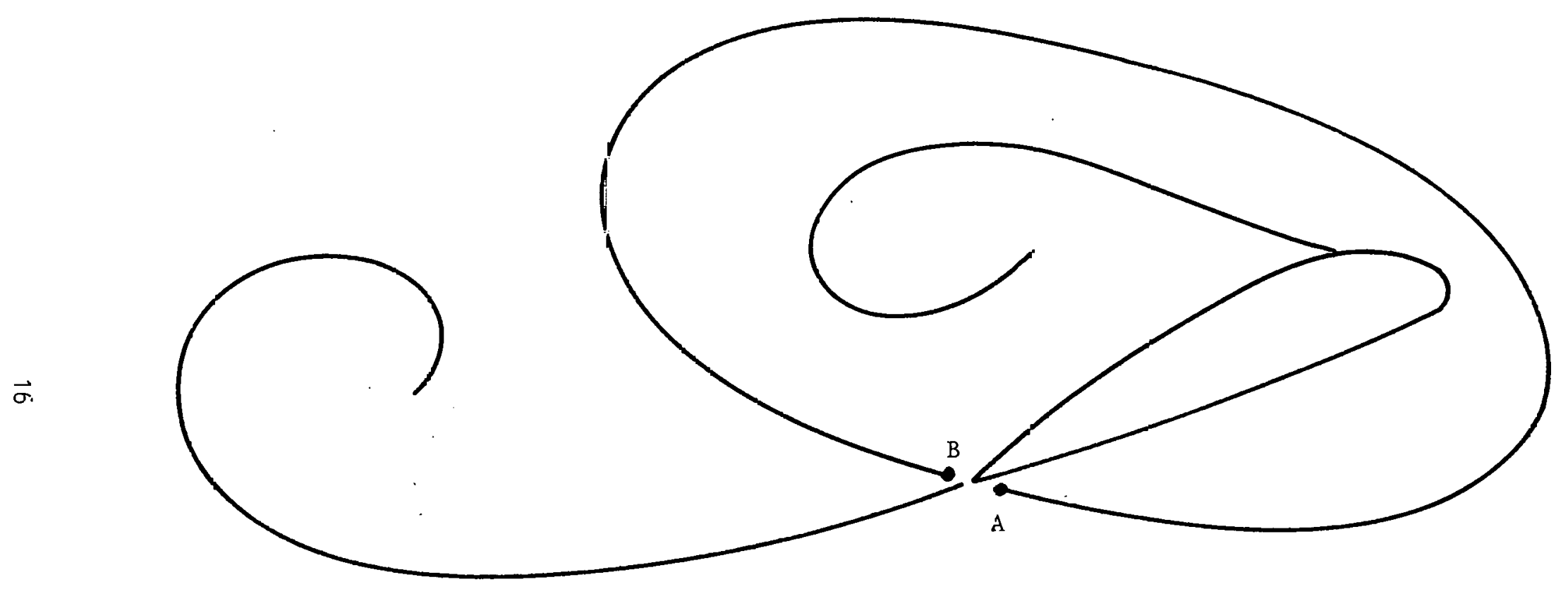

Figure 2.2.4 The Rate of Change of Fotential Jump Across the Trailing Edge 


$$
0=\frac{\partial}{\partial t}\left(\phi_{A}-\phi_{B}\right)+\frac{1}{2}\left(\nabla \phi_{A}^{2}-\nabla \phi_{B}^{2}\right)
$$

As in Eqn. 2.2.11, we may write

$$
\frac{\partial \Gamma s}{\partial t}=\frac{1}{2}\left(\nabla_{\phi}^{2}-\nabla_{\phi_{B}^{2}}^{2}\right) .
$$

Substituting into Eqn. 2.2.15 ylelds

$$
\frac{\partial \phi_{B}}{\partial t}=\frac{\partial \phi_{A}}{\partial t}+\frac{d \Gamma S}{d t} .
$$

Therefore, it is noted that behind the boundary layer separation point, there is an additional increment to $\partial \phi / \partial t$ equal to the rate of vorticity shedding from the separation point.

The same observation may be made by considering the rate of change of the potential jump across the trailing edge as described by Eqn. 2.2.13 which may be rewritten as

$$
\frac{d \phi_{u}}{d t}=\frac{d \phi_{\ell}}{d t}+\frac{d \Gamma b}{d t}+\frac{d \Gamma s}{d t} .
$$

For the case of a steady stalled airfoil, the average rates of change of $\Gamma_{b}$ and $\phi_{\ell}$ are zero, yet the $d \phi_{u} / d t$ is nonzero due to the vorticity being shed from the boundary layer separation point.

The additional contribution to $\partial \phi / \partial t$ in the separated region is important in the calculation of the pressure distribution around the airfoil. Without its inclusion, a finite pressure jump will be indicated across the two wake surfaces and erroneous values of $11 \mathrm{ft}$ and drag will result.

\subsubsection{Solution Method}

For the potential flow model described in the previous sections, the governing equation is the linear Laplace equation,

$$
\nabla^{2} \phi(\vec{r}, t)=0
$$


Through an application of Green's theorem (see Appendices C and D), it may be shown that any solution to Eqn. 2.2 .18 may be represented by integrals of sources and doublets distributed over the boundaries of the flow. Furthermore, a unique solution may be obtained utilizing surface distributions of doublets alone. The Green's function solution to Eqn. 2.2.18 is then given by

$$
\begin{gathered}
\phi(\overrightarrow{\mathrm{r}}, \mathrm{t})=\frac{1}{2 \pi} \iint_{S} \sigma \frac{\partial}{\partial v}\left[\frac{1}{\mathrm{R}}\right] \mathrm{dS} \\
\quad+\frac{1}{2 \pi} \iint_{W} \dot{\Delta \phi} \mathrm{W} \frac{\partial}{\partial v}\left[\frac{1}{R}\right] d S
\end{gathered}
$$

where $\sigma=\sigma\left(\xi_{5}, t\right)$, doublet strength distribution

$$
\begin{gathered}
\text { on } S(\vec{\xi}, t)=0 . \\
\Delta \phi^{\mathrm{W}}=\Delta \phi^{\mathrm{W}}(\vec{\xi}, t), \text { doublet strength distribution } \\
\quad \text { on } W(\vec{\xi}, t)=0 . \\
\mathrm{R}=\mid \vec{u}=\text { surface normal on } S(\vec{\xi}, t)=0 \text { or } w(\vec{\xi}, t)=0 . \\
\text { and "source" polnt, } \vec{\xi} .
\end{gathered}
$$

The body and wake doublet. strength distributions, $\sigma$ and $\Delta \phi^{W}$,

respectively, must be determined through application of the boundary

conditions. It should be noted that the infinity condition is inherently satisfied by Eqn. 2.2.19.

The surface tangency condition requires that the normal relative velocity component between the fluid and solid surfaces vanish on the surfaces. This condition may be expressed as 


$$
\begin{aligned}
& \frac{\partial n}{\partial t}+\frac{\partial \phi}{\partial n}+\vec{U}_{\infty} \cdot \vec{n}=0 \\
& \text { on } S(\stackrel{t}{r}, t)
\end{aligned}
$$

Substitution of Eqn. 2.2.19 into Eqn. 2.2.20 yields

$$
\begin{gathered}
\frac{1}{2 \pi} \iint_{S} \sigma \frac{\partial^{2}}{\partial n \partial U}\left[\frac{1}{R}\right] d S=-\left\{\frac{\partial n}{\partial t}+\vec{U}_{\infty} \cdot \vec{n}\right. \\
\left.+\frac{1}{2 \pi} \iint_{W} \Delta \phi^{W} \frac{\partial^{2}}{\partial n \partial U}\left[\frac{1}{R}\right] d S\right\} \\
\text { on } S(\vec{r}, t)=0
\end{gathered}
$$

Eqns. 2.2.21 provides a singular Friedholm integral equation of the first kind for the unknown surface doublet strength distributions. Once it has been solved subject to the remaining boundary conditions, the potential at any point in the flow field may be determined by Eqn. 2.2.19. The solution is complicated, however, by the dependency upon the wake surface locations which are also unknown.

Consider the following approach to the solution of Eqns. 2.2.21:

1) At $t=0$, let the body be started impulsively and the freestream velocity brought instantaneously to $\vec{U}_{\infty}$. For this instant, there is no wake and no contribution to the downwash on the body by the wake. A unique solution for the potential fleld may then be found through a simultaneous application of the surface tangency condition (Eqn. 2.2.21) and the Kutta condition.

2) Over the next infinitesimal time increment, assume that the resulting potential (and velocity) field are unchanged. As a result, the wake surfaces generated during that time increment 
may be predicted through the application of the Kutta and boundary layer separation conditions.

3) For the next time step, the integrals over the wake surfaces of Eqns. 2.2.21 are known and the equation may once again be solved with the Kutta condition for $\sigma$.

4) Again assuming the velocity field to remain constant over the t.ime increment, the new locations of the existing wake aurfnoes may be calculated through application of the dynamic and geometric free surface conditions. In addition, new wake surfaces are shed as before.

5) In this way, the solution proceeds in a step-by-step manner towards the steady state or periodic final result.

\subsubsection{Problem in Body Fixed Coordinates}

In the previous sections, a mathematical representation of the general motion of a finite number of rigid bodies through an ideal tluid was described. All expresslono were made with respert to an Inertial, fluid fixed reference frame, i.e., translating at $\vec{U}_{\infty}$.

We now assume that the body is rigid. As a result, the functional representations of the body surface is Independent of time when given with respect to a body fixed coordinate system. Th1s plus the fact that the guveruing Laplace equation is not explicttiy time dependent suggest that it would be advantageous to transform the problem to a body fixed coordinate system.

Consider the motion of a body through an ldeal fluld as shown in Fig. 2.2.5. We denote by $K_{0}$ or the subscript ' $O$ ', operations with respect to the fluid fixed frame. A $K$ or the absence of a subscript 


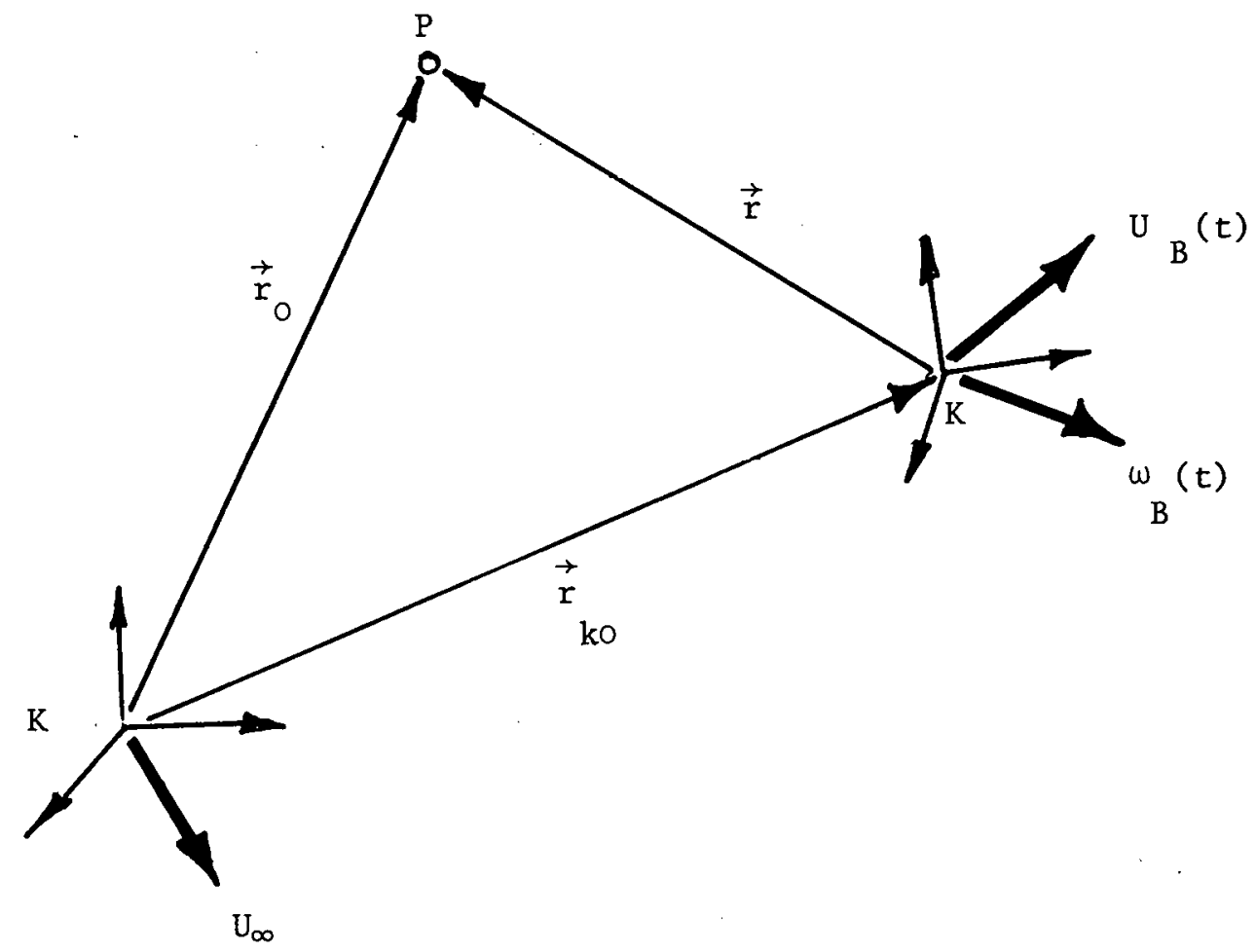

Figure 2.2.5 Inertial and Body Fixed Reference Frames 
indicates operations with respect to the body fixed frame. These coordinate systems are illustrated in Fig. 2.2.5. It should be noted that the translational velocity, $U_{\infty}$, of the $K_{0}$ frame and the translational and rotational velocities, $U_{B}$ and $\omega_{B}$, of the $K$ frame are taken with respect to an inertially fixed frame.

To establish the connection between the two coordinate systems, note that the position vectors for an arbitrary fluld fixed point $P$ for the two reference frames are related by

$$
\begin{aligned}
\overrightarrow{\mathbf{r}} & =\overrightarrow{\mathbf{r}}\left(\overrightarrow{\mathrm{r}}_{0}, t\right) \\
& =[\mathrm{T}]\left\{\overrightarrow{\mathrm{r}}_{0}-\vec{r}_{\mathrm{K}_{0}}(t)\right\}
\end{aligned}
$$

where $\stackrel{\vec{r}}{\mathrm{Ko}}_{\mathrm{K}}=\int_{0}^{t} \overrightarrow{\mathrm{U}}_{\mathrm{Ko}}(\tau) \mathrm{d} \tau+\overrightarrow{\mathrm{r}}_{\mathrm{Ko}}(0)$

and $[T]=$ coordinate transformation matrix, $i_{. e}$, for any vector $\vec{A}_{0}$, $\{\Lambda\}=[T]\left\{\Lambda_{0}\right\}$

Consider the disturbance potential field, $\phi$. Since the value of a scalar field is independent of the reference frame, we may write

$$
\phi_{n}\left(\vec{r}_{n}, t\right)=\phi(\vec{r}, t)
$$

Simflarly, for the pressure field and body surface function, it follows that

$$
\begin{aligned}
& P_{0}\left(\vec{r}_{v}, t\right) \equiv P(\vec{r}, t) \\
& S_{0}\left(\vec{r}_{0}, t\right)=S(\vec{r}) .
\end{aligned}
$$

In Eqn. 2.2.25, advantage has been taken of the fact that $S$ is independent of time with respect to the body fixed reference frame. 
Since the gradient operator defines a vector fleld, gradients from the $K_{0}$ and $K$ frames may be related by

$$
\{\nabla\}=[\mathrm{T}]\left\{\nabla_{\mathrm{o}}\right\}
$$

Then for the Laplacian,

$$
\nabla^{2}=\{\nabla\}^{\mathrm{T}}\{\nabla\}
$$

or

$$
\nabla^{2}=\left\{\nabla_{0}\right\}^{T}[T]^{T}[T]\left\{\nabla_{0}\right\}
$$

For the particular case of a transformation matrix, it may be shown that

$$
[\mathrm{T}]^{-1}=[\mathrm{T}]^{\mathrm{T}}
$$

so

$$
[\mathrm{T}]^{\mathrm{T}}[\mathrm{T}]=[\mathrm{I}]
$$

Eqn. 2.2.27 then becomes

$$
\nabla^{2}=\left\{\nabla_{0}\right\}^{T}\left\{\nabla_{0}\right\}
$$

or

$$
\nabla^{2}=\nabla_{0}^{2}
$$

To relate the time derivatives for the two reference frames, we write

$$
\frac{\partial}{\partial t_{0}}=\frac{\partial t}{\partial t_{0}} \frac{\partial}{\partial t}+\frac{\partial \vec{r}}{\partial t} \cdot \nabla
$$

But since time is unchanged by the transformation,

$$
\frac{\partial t}{\partial t_{0}}=1
$$

and

$$
\frac{\partial}{\partial t}=\frac{\partial}{\partial t}+\frac{\partial \vec{r}}{\partial t} \cdot \nabla
$$


The second term of the right hand side of Eqn. 2.2.29 is the contribution to the temporal variation due to the motion of the $K$ frame relative to the $K_{0}$ or fluid fixed frame. In a sense, it is like the convective contribution to a substantial derivative due to the fact that it represents the change of a property $Q(r)$ resulting from a variation of $r$ with respect to the fluid fixed reference frame.

Eqn. 2.2.29 may be rewritten as

$$
\frac{\partial}{\partial t_{0}}=\frac{\partial}{\partial t}+\left[\vec{U}_{\infty}-\vec{U}_{B}(t)-\vec{\omega}_{B} x \vec{r}\right] \cdot \nabla
$$

where the relative velocity between the $\mathrm{K}$ and $\mathrm{K}_{0}$ frames has been written as the difference of their velocity with respect to the inertially fixed frame.

Recall from the previous section that the governing equation for the potential flow model is

$$
\nabla_{0}^{2} \dot{\phi}_{0}\left(\vec{r}_{0}, t\right)=u
$$

where the subscripts ' $O$ ' Indicate operations with respect to the fluld fixed frame. Substitution of Eqns. 2.2.23 and 2.2.28 into Eqn. 2.2.31 ylelds

$$
\nabla^{2} \phi(\vec{r}, t)=0
$$

which is the governing equation for the body fixed problem.

The surface tangency boundary condition was written for the fluid fixed frame as 


$$
\frac{1}{\left|\nabla_{0} S_{0}\left(\vec{r}_{0}, t\right)\right|} \frac{\partial S_{0}\left(\vec{r}_{0}, t\right)}{\partial t}+\nabla_{0} \phi_{0}\left(\vec{r}_{0}, t\right) \cdot \frac{\nabla_{0} S_{0}\left(\vec{r}_{0}, t\right)}{\left|\nabla_{0} S_{0}\left(\vec{r}_{0}, t\right)\right|}=0
$$

on $s_{0}\left(\vec{r}_{0}, t\right)=0$.

Substitution of Eqns. 2.2.23, 2.2.25, and 2.2.30 into Eqn. 2.2.33 yields

$$
\begin{aligned}
\frac{1}{|\nabla S(\vec{r})|}\left\{\frac{\partial S(\vec{r})}{\partial t}\right. & \left.+\left[\vec{U}_{\infty}-\vec{U}_{B}(t)-\vec{w}_{B}(t) \times \vec{r}_{r}\right] \cdot \nabla S(\vec{r})\right\} \\
& +\nabla_{\phi} \cdot \frac{\nabla S(\vec{r})}{|\nabla S(\vec{r})|}=0
\end{aligned}
$$

or

$$
\frac{\partial \phi(\vec{r}, t)}{\partial n(\vec{r})}=-\left[\vec{U}_{\infty}-\vec{U}_{B}(t)-\vec{\omega}_{B}(t) \times \vec{r}-\frac{\partial \vec{n}(\vec{r})}{\partial t}\right] \text { on } S(\vec{r})=0
$$

where $n(\vec{r})=$ outward normal on $S(\vec{r})=0$ with respect to the body

$$
\text { fixed frame. }
$$

An expression for the pressure field in fluid fixed coordinates was given previously by

$$
P\left(\vec{r}_{0}, t\right)=P_{\infty}-\rho\left\{\frac{\partial \phi_{0}\left(\vec{r}_{0}, t\right)}{\partial t}+\frac{1}{2}\left[\nabla_{0} \phi_{0}\left(\vec{r}_{0}, t\right)\right]^{2}\right\} .
$$

This may be rewritten in body fixed coordinates as

$$
\begin{gathered}
P(\vec{r}, t)=P_{\infty}-\rho\left\{\frac{\partial \phi(\vec{r}, t)}{\partial t}+\left[\vec{U}_{\infty}-\vec{U}_{B}-\vec{w}_{B}(t) \times \vec{r}\right] \cdot \nabla \phi(\vec{r}, t)\right. \\
\left.+\frac{1}{2}\left[\nabla_{\phi}(\vec{r}, t)\right]^{2}\right\} .
\end{gathered}
$$




\subsubsection{Numerical Solution by the Collocation Method}

We now wish to develop a technique for solving Eqn. 2.2.34 with the aid of a digital computer. For this purpose, the blade and wake surfaces are descritized into $M_{B}$ and $M_{W}(t)$ elements as shown in Fig. 2.2.7. Over each surface element, the unknown doublet strength distribution is approximated with a uniform distribution of unknown magnitude. The centroids of the surface elements are identified as control points at which the surface tangency condition is satisfied exact1y.

In addition to the surface tangency condition, a Kutta condition must be applied at the trailing edge to uniquely specity the uniknown potential distribution (see Appendix A). The essential requirement of all forms of the Kutta condition is that the flow proceed smoothly from the tralling edge of the airfoll. Actual enforcement of the condition may be accomplished by specifying the direction of wake shedding, matching the upper and lower surface trailing edge pressures or by matching velocities if a steady flow. Whatever the method of application, the Kutta condition provides an additional boundary condition which serves to represent the essential consequence of viscous boundary layers in a real fluid flow.

Consider the case of an isolated body descritized into $M$ elements as shown in Fig, 2.2.7. We now have M surface tangency conditions plus the Kutta condition but only $M$ unknown doublet panel strengths, $\sigma_{i}$ so that the problem as stated is overspecifled. Elther an additional singularity of unknown strength must be added to the flow or the number of boundary conditions must be reduced. The latter approach has been followed in the present investigation. 


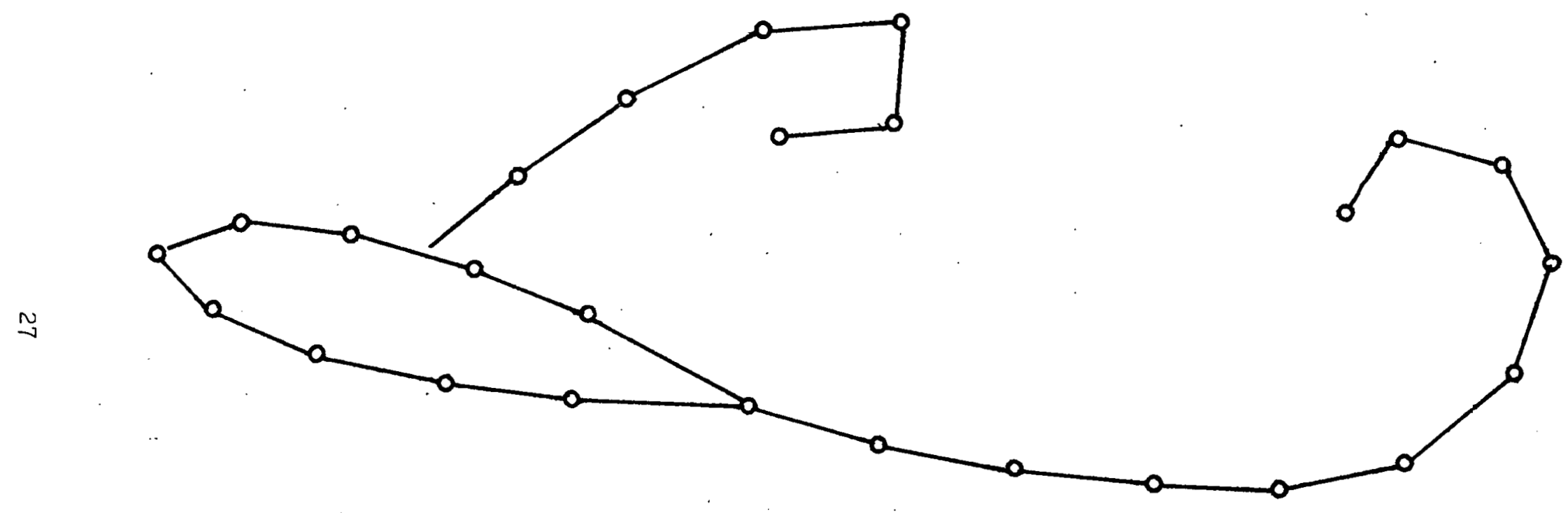

Figure 2.2.7 Finite Element Representation of Airfoil and Wake Surfaces 
Rather than applying surface tangency conditions on both the upper and lower surface elements at the trailing edge, the flow is required to be tangent to the trailing edge bisector as shown in Fig. 2.2.8. In this way, the Kutta condition is satisfied as well as approximate forms of the surface tangency conditions on the trailing edge elements. Therefore, the three boundary conditions at the trailing edge are replaced by a single one and the number of boundary conditions is $M-1$. A final condition on the unknown doubler strengths is found by aoouming that the potential jump across the trailing edge has equal contributions from the upper and lowcr clements, 1.e., $\Delta \phi_{\mathrm{TE}}=\sigma_{\mathrm{M}}{ }^{-\sigma_{1}}$ and $\sigma_{\mathrm{M}}=-\sigma_{1}=\Delta \phi_{\mathrm{TE}} / 2$.

With these approximations, the surface tangency condition of Eqn. 2.2.21 may be rewritten in matrix format as

$$
[A]\{\sigma\}=\{D\}-\left[A^{W}\right]\left\{\Delta \phi^{W}\right\}
$$

where $A_{i, j}$

- normal induced velnrity coefficient at the $1^{\text {th }}$ control point due to the $j^{\text {th }}$ source element

$$
\begin{aligned}
& =\frac{1}{2 \pi} \iint_{\delta S_{j}} \frac{\partial^{2}}{\partial n_{i} \partial u_{j}}\left[\frac{1}{R}\right] d s \\
R & =\left|\vec{r}_{i}-\vec{\xi}_{j}\right| \\
\vec{r}_{1} & =\text { position of the } i^{\text {th }} \text { cuuliol point } \\
\vec{\xi}_{j} & =\text { position nf the } j^{\text {th }} \text { source element } \\
\vec{n}_{1} & =\text { surface normal at the } i^{\text {th }} \text { control point } \\
\vec{v}_{j} & =\text { surface normal at the } j{ }^{\text {th }} \text { source element }
\end{aligned}
$$




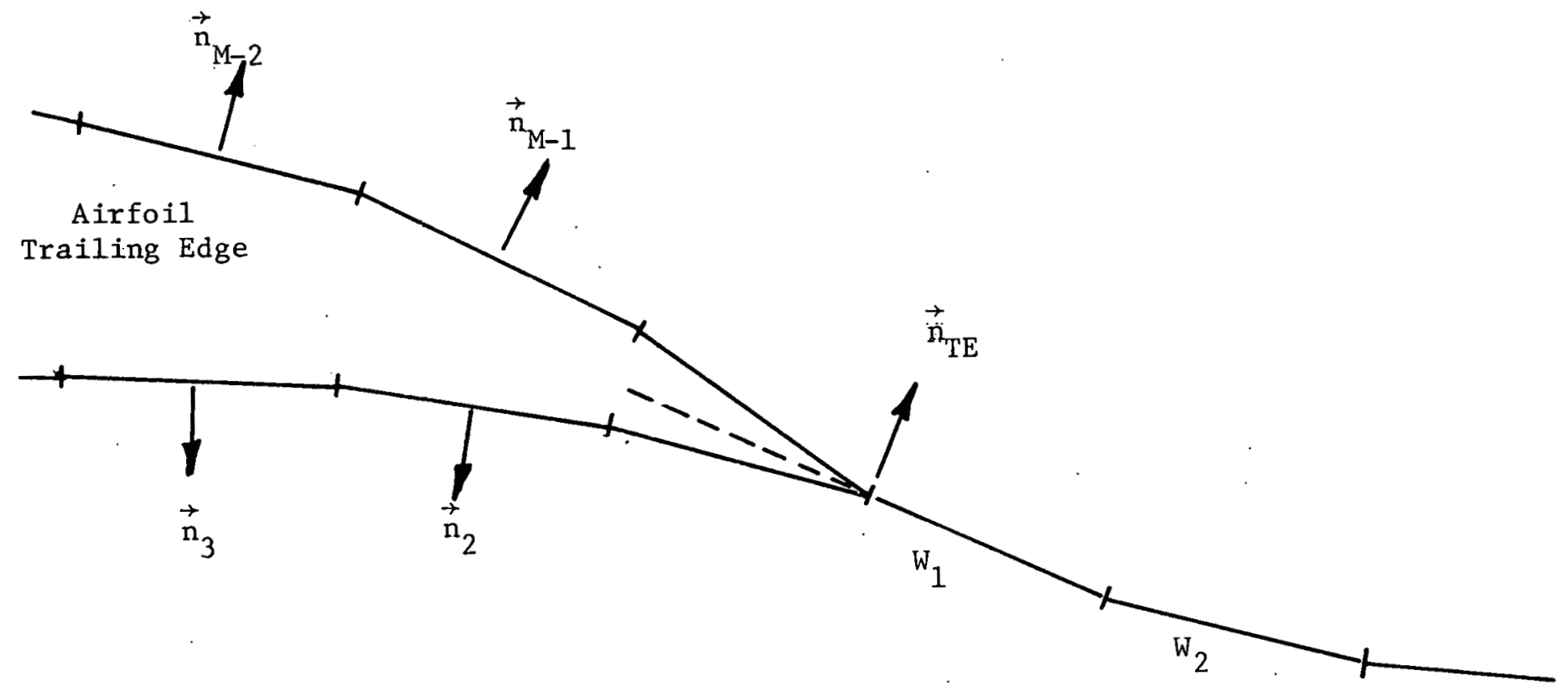

Figure 2.2.8 The Kutta Condition at the Trailing Edge 
and

$$
\begin{aligned}
\sigma_{j}= & \text { strength of the } j^{\text {th }} \text { doublet element } \\
D_{i}= & \text { normal downwash at the } i^{\text {th }} \text { control point } \\
& \text { due the freestream velocity and motion of } S \\
A_{i, j}^{W}= & \text { normal induced velocity coefficient at the } i^{\text {th }} \\
& \text { concrol polul due to the } j^{\text {th }} \text { wake source teiment } \\
\wedge \phi_{j}^{W}= & \text { strength of the } j^{\text {th }} \text { wake doublet element. }
\end{aligned}
$$

Recall that the body and freestream are started impulsively such that the wake doublet strengths, $\left\{\Delta \Phi^{w}\right\}$, are known for that instant and all later ones. Therefore, it is convenient to define

$$
\begin{aligned}
\{B\} & =\text { total downwash array } \\
& =\{D\}-\left[A^{W}\right]\left\{\Delta \phi^{W}\right\}
\end{aligned}
$$

so that Eqn. 2.2 .36 becomes

$$
[A]\{n\}=\{B\}
$$

We may then solve tho linear equation set. for the body surface doublet distribution, $\{\sigma\}$, i.e.,

$$
\{\sigma\}^{\prime}=[A]^{-1}\{R\}
$$

It should be noted that the coefficlent maliix, [A], does not change with time since the body has a fixed geometry.

Once the unknown doublet strengths have been determined, the potential for any $b$ points in the field may be found from a matrix expression of Eqn. 2.2.19,

$$
\left\{\phi_{b}\right\}=\left[C_{b}\right]\{\sigma\}+\left[C_{b}^{w}\right]\left\{\Delta \phi^{w}\right\}
$$




\subsubsection{Evaluation of the Influence Coefficients}

As noted in the previous section, the representation of a general doublet distribution on a surface element by a uniform distribution permits the definition of normal velocity and potential influence coefficlents. These were given by

$$
A_{1, j}=\frac{1}{2 \pi} \iint \delta s_{j} \frac{\partial^{2}}{\partial n_{i} \partial v_{j}}\left[\frac{1}{R}\right] d s
$$

and $C_{j} \equiv$ potential influence coefficient at $\stackrel{\vec{r}}{\mathrm{r}}_{\mathrm{b}}$ due to the $j^{\text {th }}$ source element

$$
C_{j}=\frac{1}{2 \pi} \iint \delta S_{j} \frac{\partial}{\partial \nu_{j}}\left[\frac{1}{R}\right] d S
$$

The direct evaluation of the integrals of Eqns. 2.2.40 and 2.2.41 may be avoided if we take advantage of the analogy between surface distribution of doublets and vortices. It may be shown (see Appendix D) that a general distribution of doublets may be represented by a distribution of vortices on the surface. The strength of the vortex sheet at any point is equal to the gradient of the doublet strength with vortices normal to that gradient. For the particular case of a surface element having a uniform doublet distribution, an equivalent representation is that of a vortex ring on the boundary of the element with strength equal to the element doublet strength. This is illustrated for a two-dimensional surface element in Fig. 2.2.9.

We may determine the influence coefficlents by evaluating the vortex equivalents of the doublet elements. Referring to Fig. 2.2.9, the potential influence coefficlent may be written as 


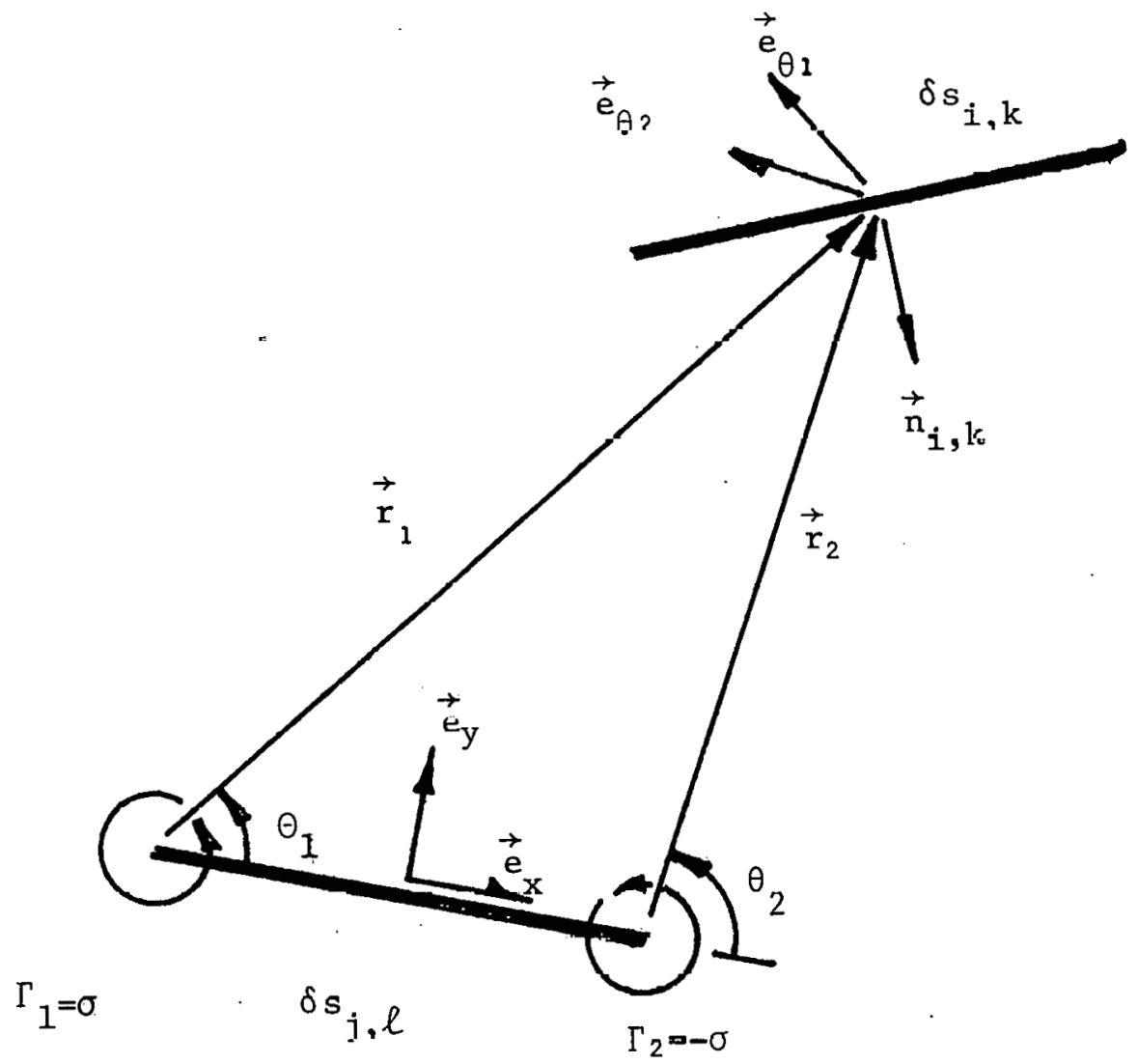

Flgure 2.2.9 Evalualion of Element Influcnec Cocfficients 


$$
\mathrm{C}_{j}=\frac{\theta_{1}}{2 \pi}-\frac{\theta_{2}}{2 \pi}
$$

$$
=\frac{1}{2 \pi}\left[\tan ^{-1}\left(\frac{\vec{r}_{1} \cdot \vec{e}_{y}}{\vec{r}_{1} \cdot \vec{e}}\right)-\tan ^{-1}\left(\frac{\overrightarrow{r_{2}} \cdot \vec{e}}{\vec{r}_{2} \cdot \vec{e}}\right)\right]
$$

For the normal velocity influence coefficient, we may write

$$
\begin{aligned}
A_{1, j} & =\left[\frac{1}{2 \pi} \frac{\vec{e}_{\theta_{1}}}{\left|\vec{r}_{1}\right|}-\frac{1}{2 \pi} \mid \frac{\vec{e}_{\theta_{2}}}{\left|\vec{r}_{2}\right|} \cdot \vec{n}_{i}\right. \\
& =\frac{1}{2 \pi}\left[\frac{\vec{e}_{z} \vec{r}_{1}}{r_{1}{ }^{2}}-\frac{\vec{e}_{z} \overrightarrow{x r}_{2}}{r_{2}{ }^{2}}\right] \cdot \vec{n}_{i}
\end{aligned}
$$

It should be noted that all of the vector quantities of Eqns. 2.2.42 and 2.2.43 are given with respect to the element coordinate system 111ustrated in Fig. 2.2.8.

\subsubsection{Calculation of Airloads}

In Appendix A, it is shown that the pressure at any point in an Irrotational, ideal flow may be found with the unsteady Bernoulli equation,

$$
P=P_{\infty}-\rho\left\{\frac{\partial \Phi}{\partial t}+\frac{1}{2}(\nabla \Phi)^{2}\right\}
$$

One should recall that Eqn. 2.2.44 is valid for a fluid fixed coordinate system. In Section 2.2.4, the equivalent expression for a body fixed coordinate system was written as

$$
P=P_{\infty}-\rho\left\{\frac{\partial \Phi}{\partial t}+\left(\vec{U}_{\infty}-\vec{U}_{B}-\vec{w} \times \vec{r}\right) \cdot \nabla \Phi+\frac{1}{2}(\nabla \Phi)^{2}\right\}
$$


Eqn. 2.2.45 may be rewritten in a more convenient form for numerical computation by expressing $\nabla \phi$ as

$$
\nabla \Phi=\nabla_{\mathbf{s}} \Phi+\frac{\partial \Phi}{\partial \mathrm{n}} \stackrel{\overrightarrow{\mathrm{e}}}{\mathrm{n}}_{\mathrm{n}}
$$

where $\nabla_{s} \equiv$ surface gradient. In addition, recall that the surface tangency condition was written in body fixed coordinates as

$$
\frac{\partial \Phi}{\partial \mathbf{n}}=-\left(\vec{U}_{\infty}-\vec{U}_{B}-\vec{w}_{B} \times \vec{r}\right) \cdot \vec{n}
$$

With Eqn. 2.2.46, Eqn. 2.2.45 may be expanded to yield

$$
\begin{aligned}
P=P_{\infty} & -\rho\left\{\frac{\partial \Phi}{\partial t}+\left(\vec{U}_{\infty}-\vec{U}_{B}-\vec{w}_{B} \times \vec{r}\right) \cdot \nabla_{s} \Phi\right. \\
& +\frac{\partial \Phi}{\partial n}\left(\vec{U}_{\infty}-\vec{U}_{B} \vec{w}_{B} \times \vec{r}\right) \cdot n \\
& \left.+\frac{1}{2}\left(\nabla_{s} \Phi\right)^{2}+\frac{1}{2}\left(\frac{\partial \Phi}{\partial n}\right)^{2}\right\}
\end{aligned}
$$

By substituting Eqn. 2.2.47 into Eqn. 2.2.48, we arrive at

$$
\begin{aligned}
& P \equiv P_{\infty}-\rho\left\{\frac{\partial \Phi}{\partial t}+\left(\vec{t}_{\infty}-\vec{t}_{H}-\overrightarrow{-11}_{K} \times \vec{r}\right) \cdot \nabla_{S} \Phi\right. \\
& -\frac{1}{2}\left[\left(\overrightarrow{I I}_{\infty}-\vec{U}_{B}+\overrightarrow{n I}_{B} \times \vec{r}\right) \cdot \vec{n}^{2}\right]^{2} \\
& \left.+\frac{1}{2}(\nabla s)^{2}\right\}
\end{aligned}
$$

Eqn, 2.2.49 provides the advantage of reducing the computation of the gradient of the discribution potential to the computation of 1 ts ourface gradient. With Eqn. 2.2.49, the airloads on the body surface may be calculated by integrating the pressure force vector components over the surface. 


\subsection{The Boundary Layer Mode1}

There were several general requirements which had to be met by the boundary layer model used in this work. The model had to be able to predict both laminar and turbulent portions of the boundary layer flow as we11 as the location of the transition region between them as indicated in FIg. 2.3.1. Unsteady effects had to be an intrinsic part of the formulation since they have been shown to contribute to stall delay in a signiflcant manner [see McCroskey and Phillippe (1975)] • The boundary layer model had to also be capable of making reasonably accurate predictions in the vicinity of separation so that appropriate separation criteria could be applied. In addition, calculation times had to be reasonable.

\subsubsection{Overview of Unsteady Boundary Layer Codes}

Several investigators have made extensive calculations using unsteady turbulent boundary layer codes in recent years, e.g., Nash, Carr, and Singleton (1975); Dwyer and McCroskey (1971); Telionis (1975); Daneshyar and Mugglestone (1978); and Lyrio, Ferziger, and K1ine (1981). Notable among the codes which have been developed are the several vartations of a differential boundary layer model due to Nash, et al. (1978). This model met most of the general requirements in that it predicts both laminar and turbulent flow, includes unsteady effects, and is capable of predicting flow in the vicinity of separation. In addition, the model has been applied to dynamic stall problems and has been shown to give good results according to McCroskey and Phillippe (1975). While the computational time required 


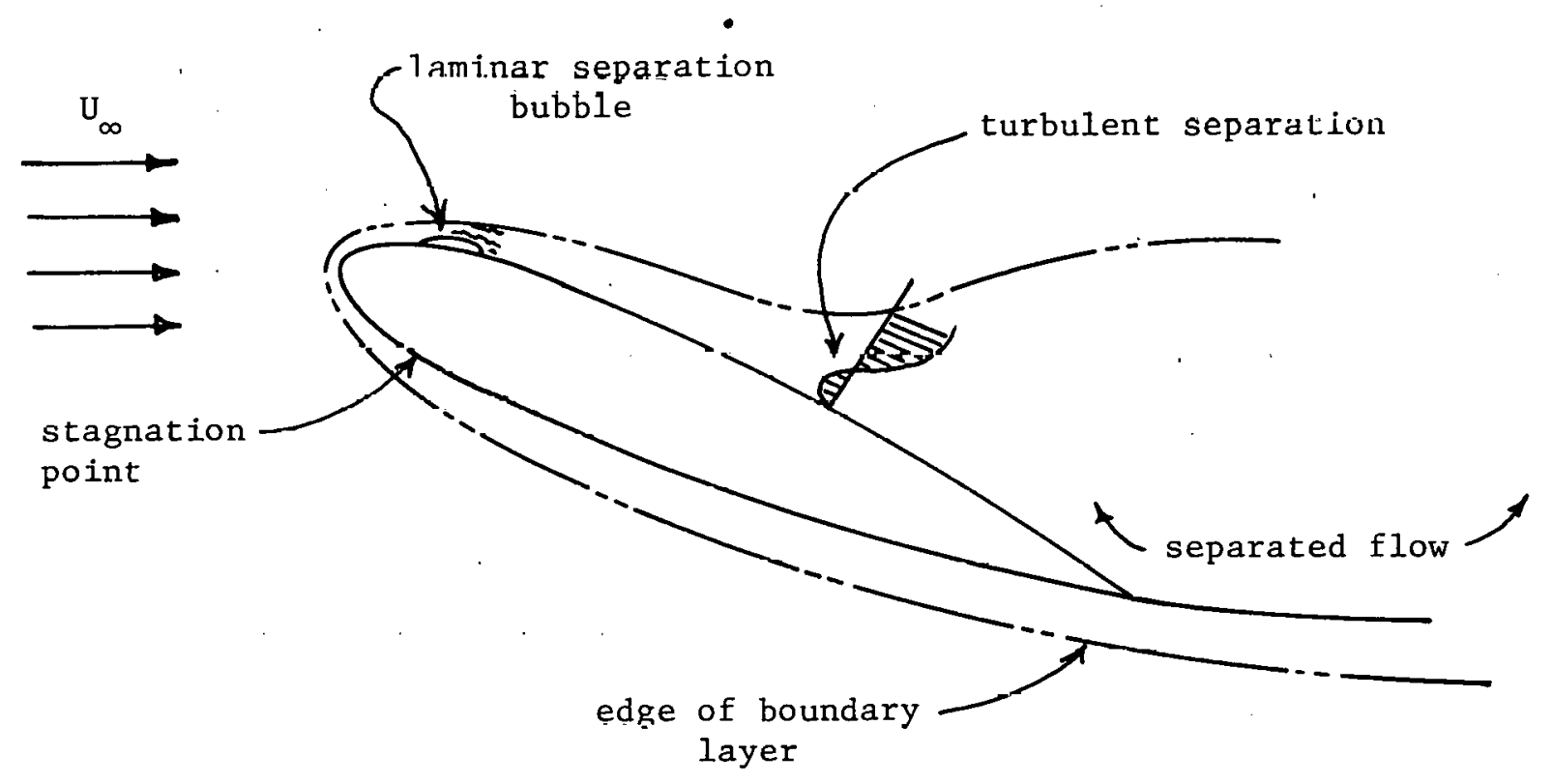

Fig. 2.3.1 Boundary Layer Characterlstics of an Airfoil at High Incidence 
for this method is not excessive in comparison with other differential boundary layer codes, it was felt that an integral technique might be more appropriate due to the large number of boundary layer calculations required.

Only very recently have integral techniques for unsteady turbulent boundary layers appeared in the 1iterature. The method due to Daneshyar and Mugglestone (1978) utilizes the unsteady momentum integral equation along with the entrainment equation and a skinfriction equation derived from the Coles (1956) velocity profile. Other assumptions and linearizations which are a part of this formulation restrict its use to cases of small amplitude periodic fluctuations of the freestream. Very recently, however, Lyrio, Ferziger, and Kline (1981) formulated and tested a similar integral technique which gives excellent results for the steady turbulent flows of Tillman, Herring and Norbury; Stratford, Samuel and Joubert [see Coles and Hirst (1968) for these four flows]; Kim (1980); Simpson and Strickland (1977); and Wieghardt [see KIm (1980)]. More Importantly, this method predicts the unsteady turbulent boundary layer data of Karlson (1959), and Ho!nevi1.1e, et al. (1979), and comparec well with the finite difference methods of McCroskey and Philippe (1975), and Singleton and Nash (1974) whlle being an order of magnitude faster.

\subsubsection{Present Boundary Layer Model}

The turbulent boundary layer analysis used in the present work is essentially that due to Lyrio et al. (1981). This method gives good results prior to actual separation of the boundary layer as evidenced in Figures 2.3.2 and 2.3.3. Predictions near separation as well as 

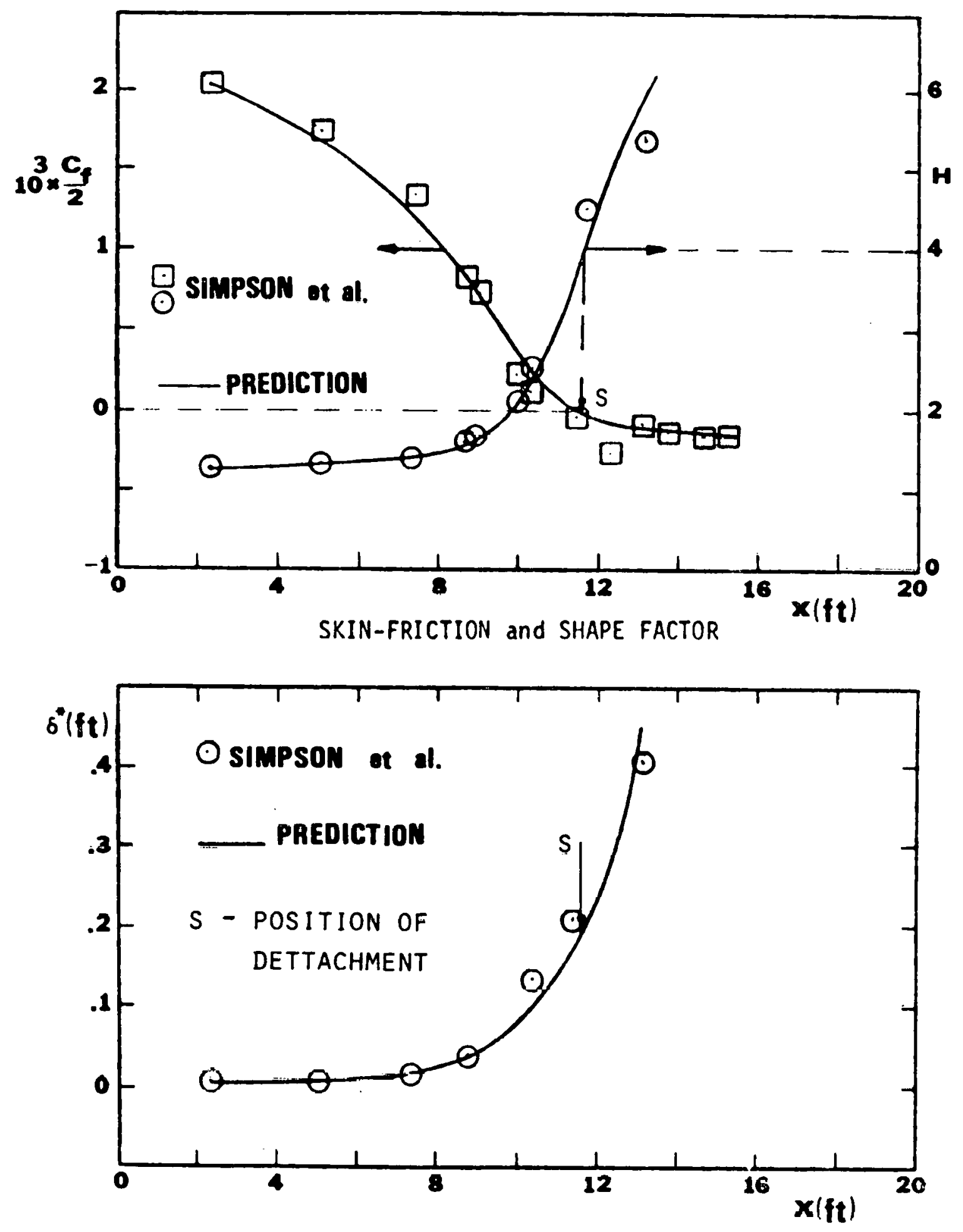

DISPLACEMENT THICKNESS

Fig. 2.3.2 Typical Predictive Capability of Lyrio et al. (1981) Boundary Layer Code for Steady Separating Flows 

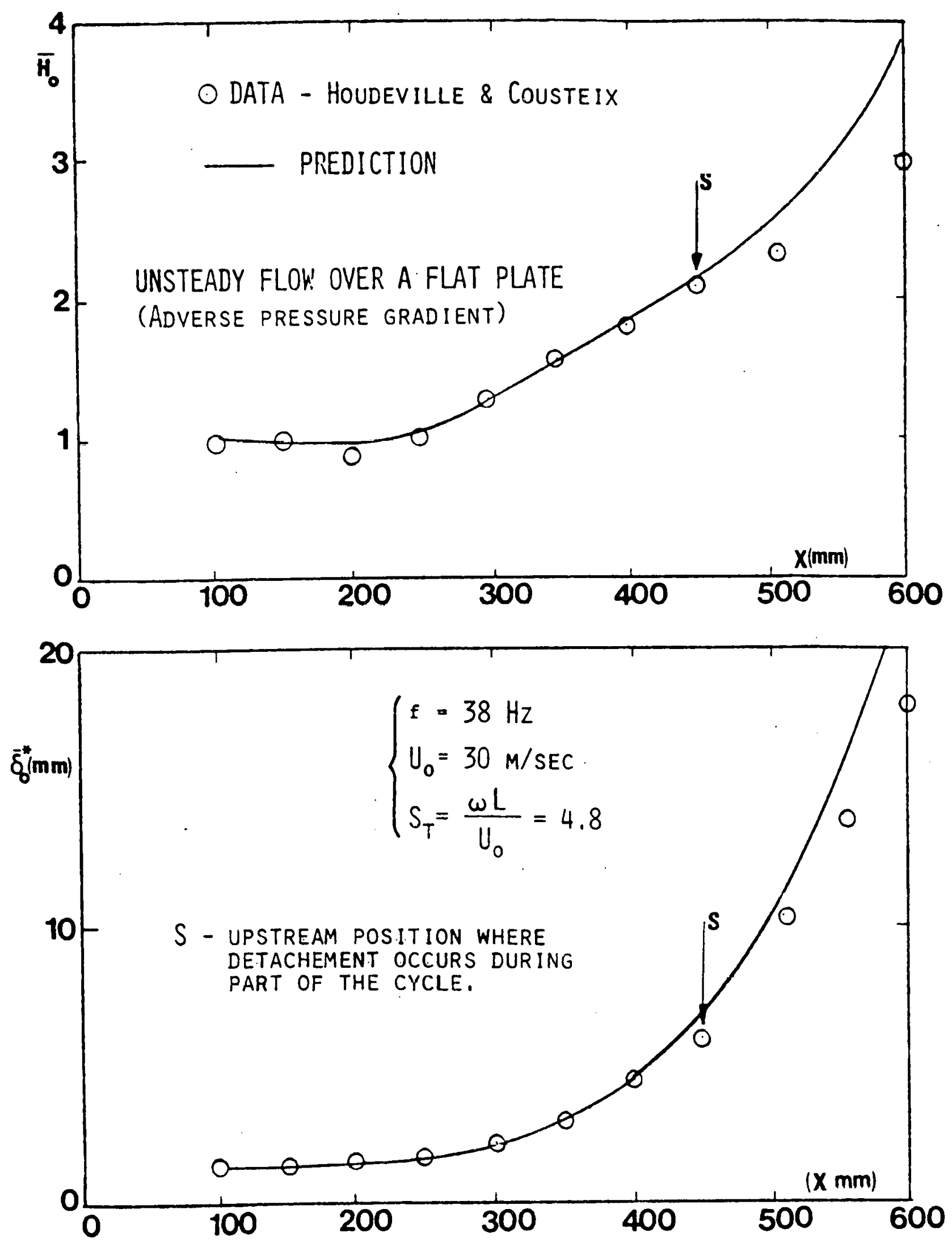

Fig. 2.3.3 Typical Fredictive Capability of Lyr1o et a1. (1981) Boundary Layer Code for Unsteady Separating Flows 
downstream of separation tend to be very sensitive to freestream veloc1ty gradients as evidenced by the present author's experfence with the analysis. The formulation for the laminar portion of the boundary layer is based upon an extension of Thwaltes method [see Cebecci and Bradshaw (1977)]. The major extension of Thwaites method is the inclusion of unsteady terms in the momentum integral equation.

The unsteady momentum integral equation which is valid for both laminar and turbulent flow can be written as

$$
\frac{1}{U_{e}^{2}} \frac{\partial}{\partial t}\left(U_{e} \delta^{*}\right)+\frac{\partial \dot{\theta}}{\partial x}+\frac{1}{U_{e}} \frac{\delta U_{e}}{\partial x}\left(2 \theta+\delta^{H}\right)=\frac{C_{f}}{2}
$$

where $U_{e}, \theta, \delta^{*}$ and $C_{f}$ are the freestream veloctty, momentum thickness, displacement thickness, and friction factor, respectively. For the laminar formulation, a pressure gradient parameter, $\lambda$, is defined as

$$
\lambda=\frac{\theta}{\widetilde{U}_{e}} R_{\theta}\left(\frac{\partial U}{\partial x}+\frac{e}{U_{e}} \frac{\partial U}{\partial t}\right)
$$

where $R_{\theta}$ is the Reynolds number based on $U_{e}$ and $\theta$. In order to obtain 2 solution th the laminar case a connection between $C_{f} / 2, \theta$, and $\delta^{*}$ must be made. The following correlations were obtalned based on wedge flow solutions which make this connection:

$$
\begin{aligned}
& \frac{C_{f}}{2}=\frac{1.91-4.13 \Lambda}{R e} \\
& H=(0.680-0.922 \Lambda)^{-1} \\
& \Lambda=0.325-0.130 \lambda \mathrm{H}^{?}
\end{aligned}
$$


Here, $\mathrm{H}$ is the usual shape parameter, $\delta * / \theta, \Lambda$ is the blockage factor $\delta^{*} / \delta$, and $\mathrm{Re}^{*}$ is the Reynolds number based on $\mathrm{U}_{\mathrm{e}}$ and $\delta^{*}$.

The turbulent boundary layer model of Lyrio, Ferziger, and K1lne utilizes the entrainment equation given by

$$
\frac{1}{U_{e}} \frac{\partial}{\partial x}\left[U_{e}\left(\delta-\delta^{*}\right)\right]=F
$$

The entrainment function $F$ is calculated from the following auxiliary equations

$$
\begin{gathered}
\frac{d F}{d x}=\frac{.025}{\delta}(F e-F) \\
F e=4.24 \operatorname{Ke}\left(\frac{\Lambda}{1-\Lambda}\right) \cdot 916 \\
\operatorname{Ke}=.013+.0038 e^{-\beta / 15} \\
\beta=\frac{\delta^{*}}{\tau_{w}} \frac{d P}{d x}
\end{gathered}
$$

where $t_{w}$ is the wall shearing stress and $d P / d x$ is the streamwise pressure gradient. Shape factor relationships are obtained from the following general velocity profile.

$$
\begin{aligned}
& \frac{U}{U_{e}}=1+V_{T} \ln \left(\frac{y}{\delta}\right)-V_{B} \cos ^{2}\left(\frac{\pi y}{2 \delta}\right) \\
& V_{T}=\frac{1}{.41} \sqrt{\frac{C_{f}}{2}}\left(\operatorname{sgn} C_{f} / 2\right) \\
& V_{B}=2\left(\Lambda-V_{T}\right) \\
& \frac{C_{f}}{2}=\frac{\tau_{W}}{\rho U_{e}^{2}}
\end{aligned}
$$


where $U$ is the velocity in the boundary layer at a distance $y$ from the wall and $\rho$ is the fluid density. The skin friction law is given by

$$
\frac{C_{f}}{2}=.051|1-2 \Lambda|^{1.732}\left(\frac{\stackrel{*}{R}}{\Lambda}\right)^{-.268} \operatorname{sgn}(1-2 \Lambda)
$$

\subsubsection{Transition and Separation}

Transition of the laminar boundary layer (found near the forward stagnation point of the airfoll) to a turbulent boundary layer can be triggered in one of several ways. In the first case, a separation bubble can be formed near the leadlug edge which conoloto of a laminar separation and turbulent transition with subsequent reattachment. In some cases the shear layer may not reattach. In the present model the streamwise extent of the separation bubble is assumed to be small and a turbulent boundary layer is assumed to begin at the point of laminar separation given by a value of $H \geqslant 4.0$. A model for the separation bubble has been proposed and tested by Roberts (1980) and Arena and Mueller (1980) and should eventually be included in the boundary layer code. In the absence of a laminar separation bublle, transition may oscur hy virtile of laminar instabilities which can be predicted using the criteria due to Cebecci and Smith (1974)

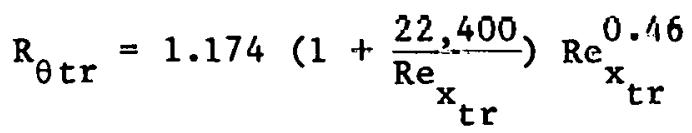

Here $k_{\theta}$ and $K_{e} e_{x}$ are Reynolds numbers based on the momentum thlckuess and distance from the forward stagnation point, respectively. As pointed out by McCroskey (1975), this latter type of instability occurs only at high Reynolds numbers and low angles of attack. 
Separation criterla assoclated with the unsteady boundary layer is more complicated than for the steady case in which it is generally assumed that separation occurs at a position of zero wall shear stress. For example, it is evident that no separation occurs from a flat plate oscillating parallel to its own surface in an otherwise still fluid even though the wall shear stress goes to zero twice during each cycle. Several Investigators have examined the Moore (1958)-Rott (1956)-Sears (1956) model and have concluded that if properly interpreted, it ylelds an unsteady boundary layer separation criteria. Basically, the model requires that the shear stress must vanish at some point within the boundary layer and, in addition, the velocity relative to a coordinate frame moving with the separation point must vanish at the same location. This criteria is also consistent with the findings of Nash and Patel (1975) in that they conclude that separation will occur if and only if the typical reversed flow velocities (which their model calculates) exceed the rate of penetration of the reversed flow into the oncoming boundary layer. An alternate separation criteria is based on monftoring any rapid increase in the various boundary parameters such as the displacement thickness $\delta^{*}$ or the $v$ component of velocity normal to the surface. Nash and Patel (1975), in fact, use a crude criteria to enable them to locate the approximate location of separation by noting the region where $\delta * / C>0.1$ where $C$ is the alrfoll chord length. In the work of Lyr1o, Ferzlger, and Kline the "fully developed" separation point is found to occur when $\Lambda=0.5$. The "intermittent" separation point occurs prior to the "fully developed" separation point. The Intermittent acparation point occurs when 


$$
H \geqslant \frac{2-\Lambda}{1-\Lambda}
$$

according to the Sandborn (1961) correlation. the intermittent separation point is much more reliably predicted than the fully developed separation point and was thus used as the point of introduction of the nascent vortex in the present work. The exact point in the separation region at which the nascent vortex should be introduced for optimum results is presently unknown. Further study regarding this detall should be undertaken.

\section{3 .4 Numcrical Solution of Boundary Layer Equatinns}

Numerical solutions are obtained in the present model using an explicit finfte difference formulation of Eqn. 2.3.1. For the laminar formulation equations $2.3 .1,2.3 .2$, and 2.3 .3 can be cast in the following form:

$$
\text { A } \frac{\partial \delta^{*}}{\partial x}-B
$$

This equation is integrated over an interval $\Delta \mathrm{x}$ using a fourth order Runge-Kutta method to yleld the variation of $s^{*}$ as a function of $x$ uL a given time step. Time derivatives in the coefficient $\dot{B}$ are obtained from backward differences while derivatives with respect to $x$ are obtained from forward differences. These derivatives are held constant over the integration interval $\Delta x$. The integration interval $\Delta x$ is further subdivided into at least elght sublatervals. For the turbulent formulation using equations $2.3 .1,2.3 .4,2.3 .5,2.3 .6$, and 2.3.7 a pair of simultaneous equations results which can be symbolized as: 


$$
\left[\begin{array}{ll}
A_{11} & A_{12} \\
A_{21} & A_{22}
\end{array}\right]\left\{\begin{array}{l}
\frac{\partial \delta^{*}}{\partial x} \\
\frac{\partial \Lambda}{\partial x}
\end{array}\right\}=\left\{\begin{array}{c}
B_{1} \\
B_{2}
\end{array}\right\}
$$

A fourth order Runge-Kutta method is used to yield simultaneous values of $\delta^{*}$ and $\Lambda$ as a function of $x$.

In the work completed to date the time dependent terms in the code have been suppressed such that the solution obtained is of a quasi-steady nature. 


\section{CALCULATION RESULTS}

Contained in the following sections are calculation results illustrating the current capability of DYNA2 to predict steady and unsteady, attached flows and steady, separated flows over two-dimenslonal airfoils. Possible enhancements to the model which would improve the accuracy of those calculations are outlined.

\subsection{Steady, Attached Flows}

Figures 3.1 .1 through 3.1 .4 are representative of the capability of DYNA2 to predict the pressure distributions on two-dimensional bodies resulting from steady flows without boundary layer separation. The calculation results presented are for the cases of a cylinder and typical turbulent and laminar afrfolis. The model does an excellent job for the cases of the cylinder and turbulent alrfoll but is less successful for the laminar atrioll.

Figures 3.1 .3 and 3.1 .4 illustrate calculation results for the same laminar airfoll at ldentical angles of attack. The only difference in the Lwu calculations is a slight repositioning of the doublet panels near the nose. The sensitivity of the calculations to this modification is a consequence of the use of flat, uniform strength doublet panels as the basic surface modeling elements. Since only one degree of freedom (the doublet panel etrength) is allowed for each element by this representation, there can be only one boundary condition enforced on each element. The cholce made for DYNA2 is a surface tangency condition applied at the centrold of the elements. A higher order doublet strength distribution on the panels would allow extra degrees of freedom which could be utilized to enforce 


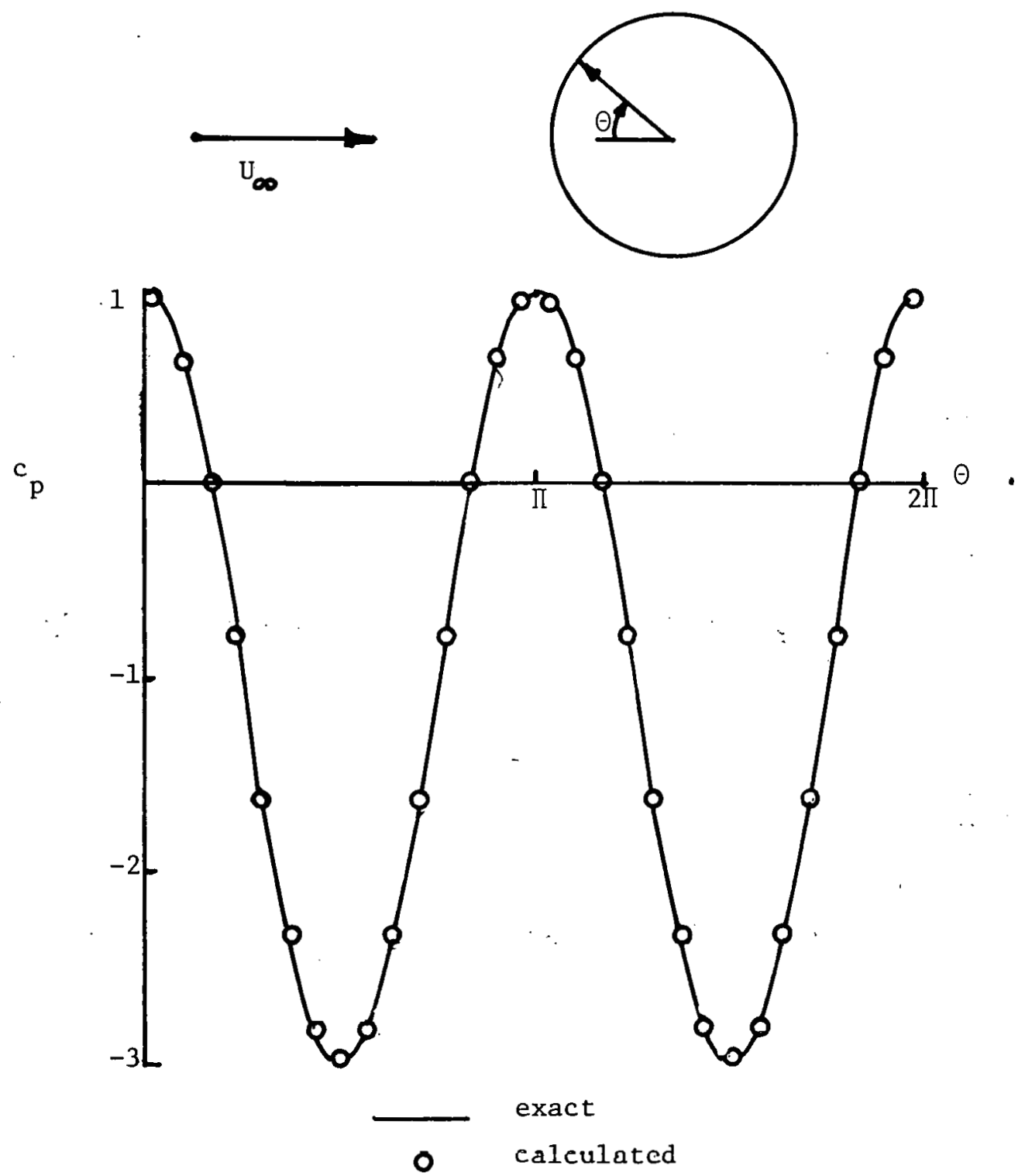

Figure 3.1.1 Pressure Distribution on a

Two-Dimensional Cylinder 


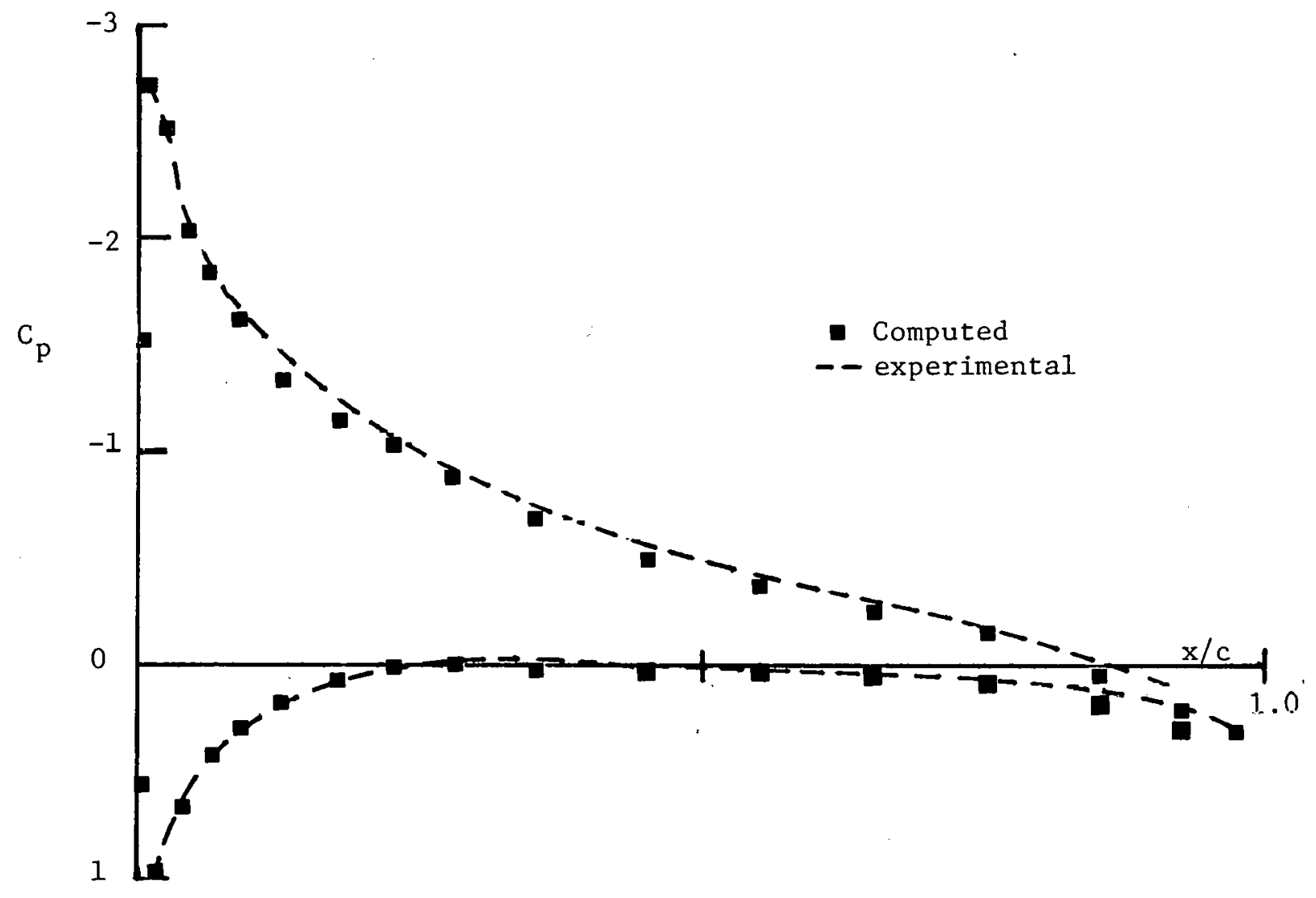

Figure 3.1.2 Pressure Distribution on a NACA 0075 Airfoil at $6^{\circ}$ Angle of Atrack 


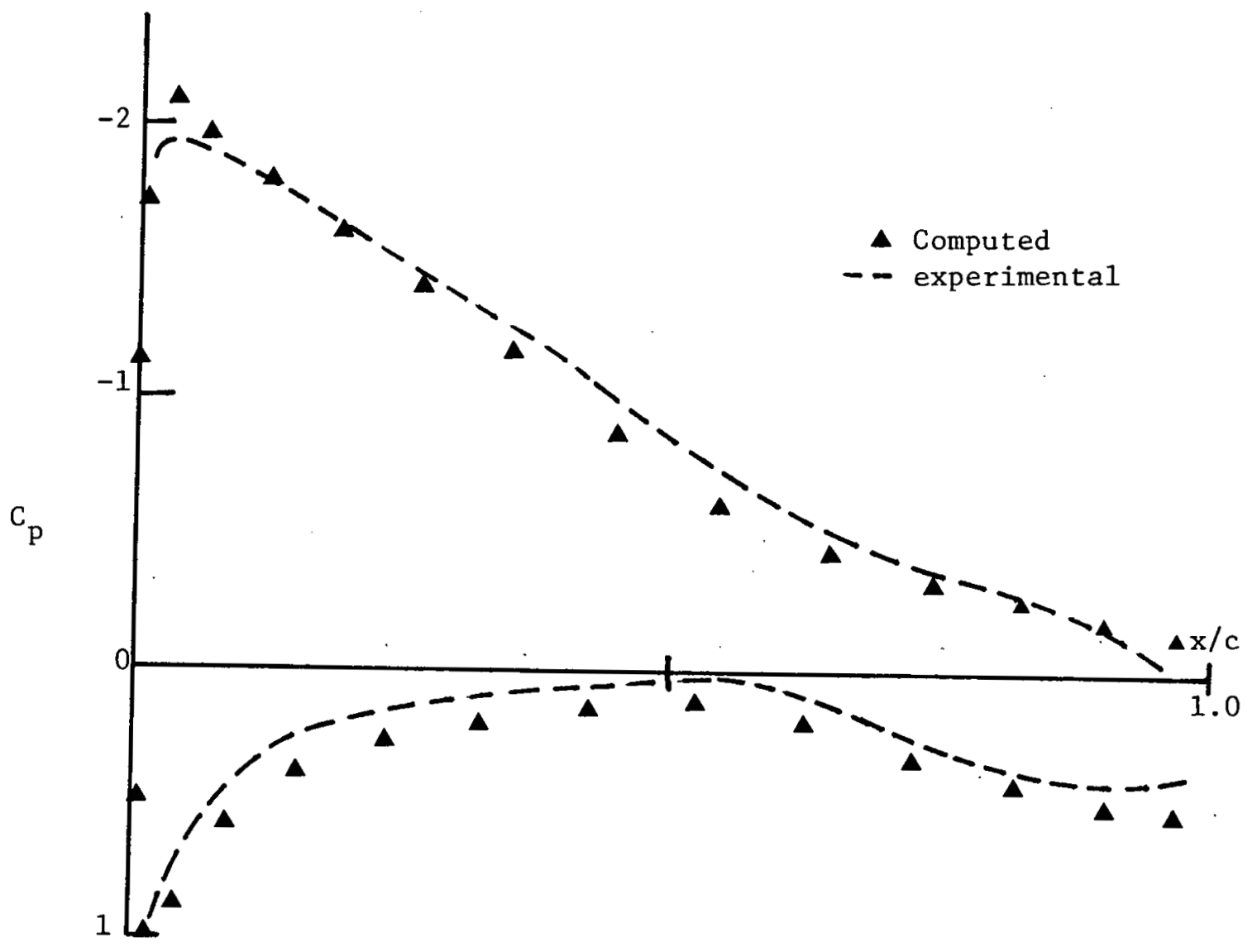

Figure 3.1.3 Pressure Distribution on a Laminar Airfoil at $6.1^{\circ}$ Angle of Attack 


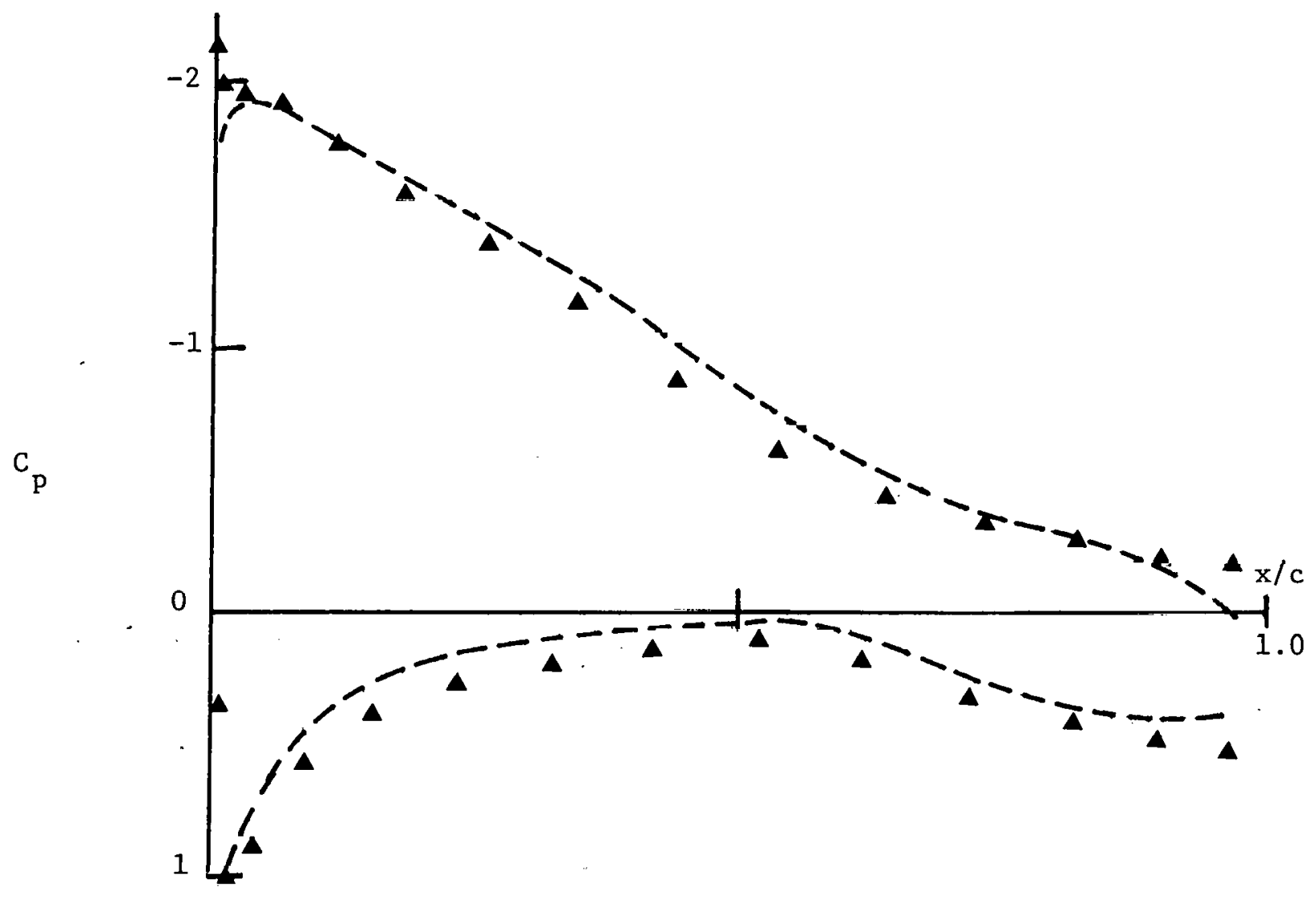

Figure 3.1.4 Same as Figure 3.1.3 Except W1th S11ghtly Modified Leading Edge Modeling 
additional boundary conditions. These might include multiple surface tangency conditions on individual elements and a requirement for continufty of the surface fluid velocity between adjacent panels.

The use of planar surface elements causes the centrolds of the elements to actually be below the true curved alrfoil surface in most cases. For turbulent airfoils and the majority of the surface of laminar airfolls, this presents no problem since the deviation will be small if the curvature is small. However, difficulties can arise near the leading edge of laminar afrfoils where the radius of curvature is typically small and the rate of change of fluid velocity with respect to a surface coordinate is large. Many, very small elements are required to adequately model regions such as this and there is little tolerance in their positioning. The situation could be improved by utilizing curved doublet panels which would more closely follow the true airfoll surface.

One disadvantage of using curved, multiple degree of freedom doublet panels as the basic surface modeling element is that the corresponding influence coefficlents can not be evaluated in closed form. Their calculation through a numerical integration scheme would increase the computation thiue requirments significantly as compared to the planar, uniform strength elements. However, on the basis of the current calculations, it appears that the more complex elements are necessary to achleve a satisfactory degree of flexibility and reliability in calculations for any arbitrary alrfoll which one might wish to consider.

\subsection{Steady, Attached Flows}

Figures 3.2.1 through 3.2.4 are typical of calculations for nonseparated flows over airfolls involved in unsteady motions. Figure 3.2 .1 


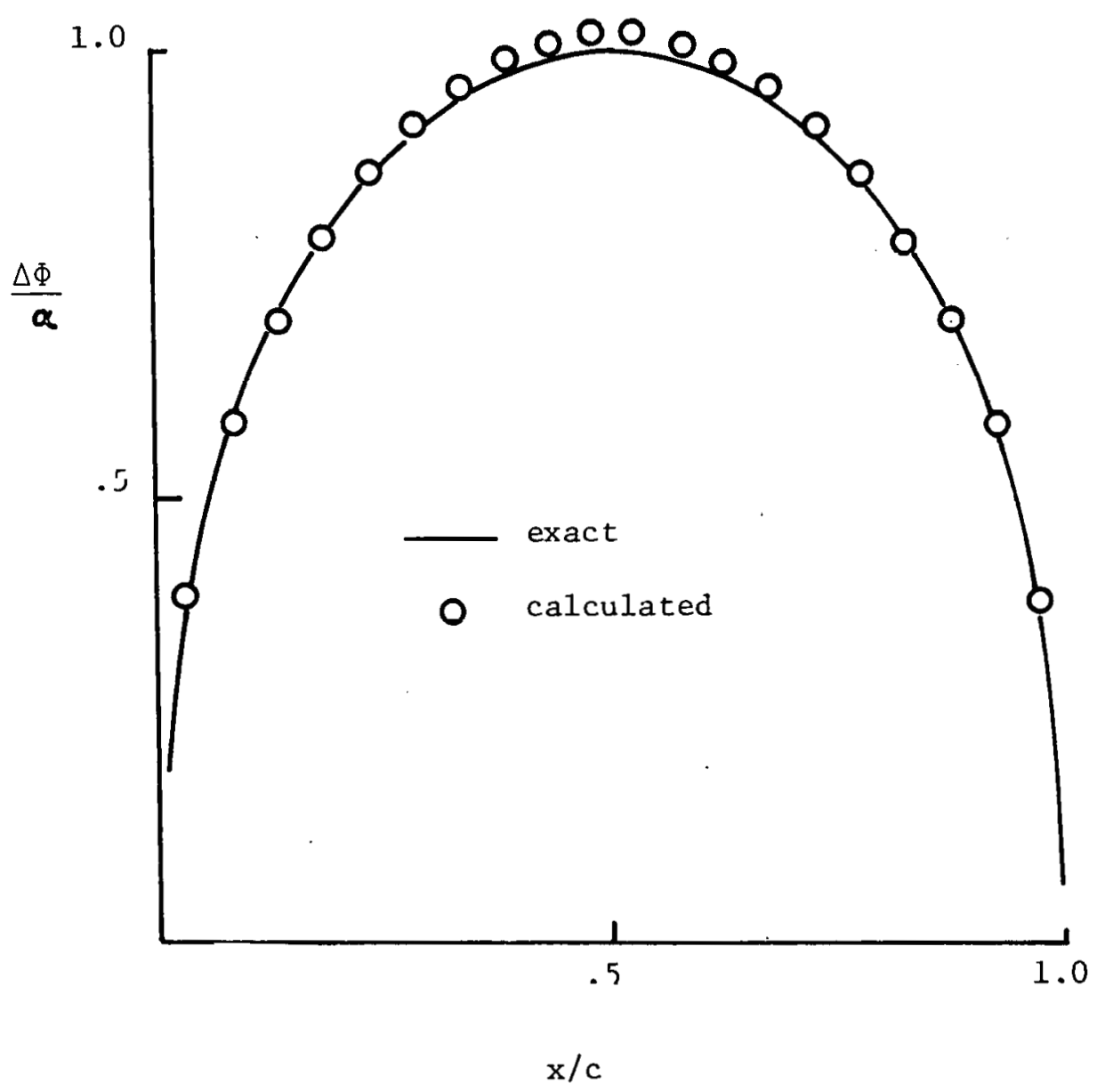

Figure 3.2.1 Potential Jump Distribution on an Impulsively started Flat Plate 


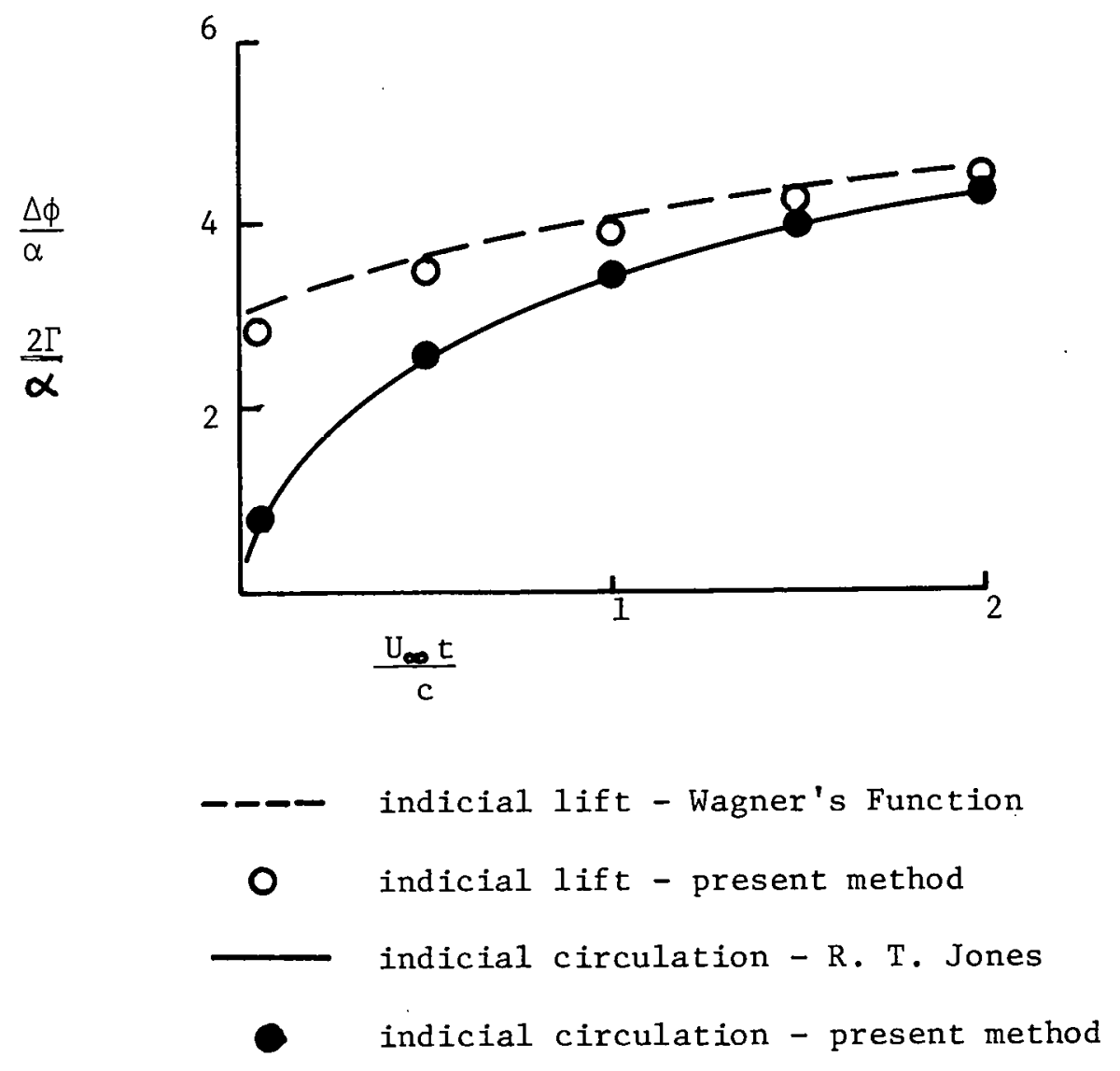

Figure 3.2.2 Impulsively Started Flat Plate Airfoil 


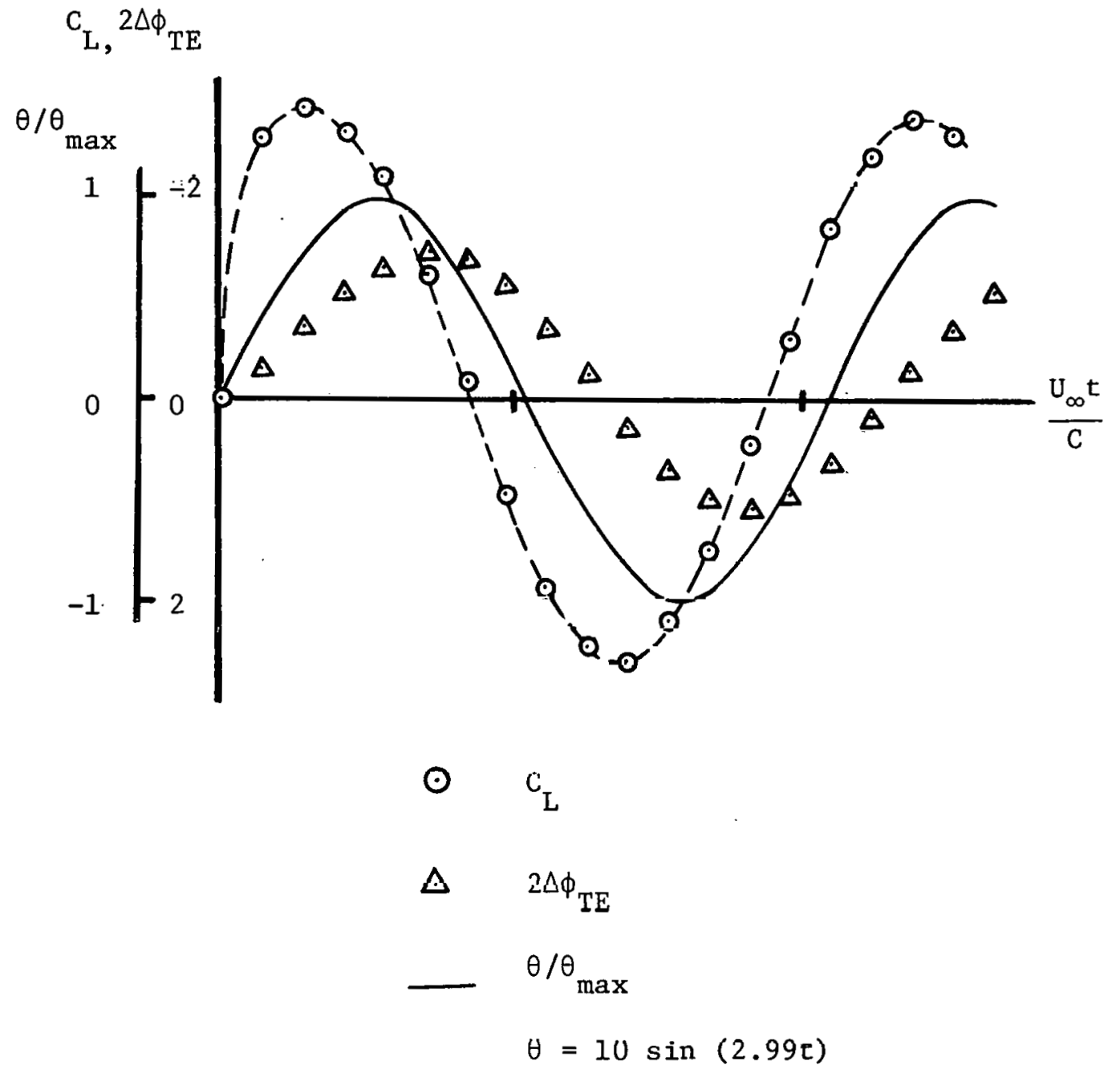

Figure 3.2.3 Variation of Lift and Circulation on an Oscillating NACA 0015 Airfoil 


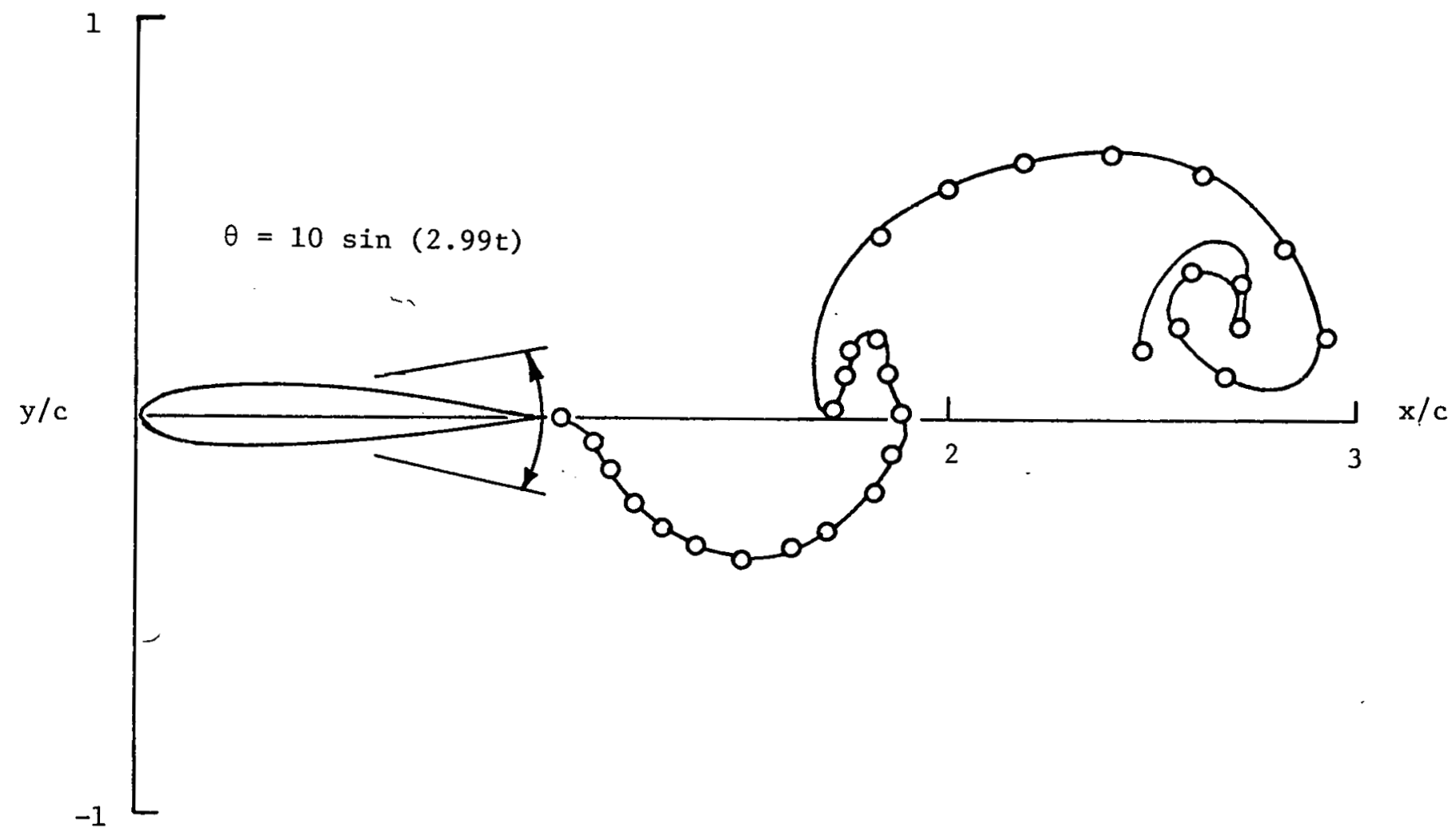

3.2.4 Wake Geometry Behind an Oscillating NACA 0015 Airfoil 
presents a comparison between the computed and exact potential jump distributions over an impulsively started flat plate alrfoll at the starting instant. Figure 3.2.2 illustrates the subsequent development of the circulation and lift.

Figures 3.2 .3 and 3.2 .4 present the results of calculations for a NACA 0015 airfoil oscillating in pitch. From Figure 3.2 .3 , it is noted that the maximum lift leads the maximum angle of attack due to the apparent mass effects. The sectional circulation, however, lags behind the angle of attack as a result of the downwash produced by the vorticity in the wake. The wake geometry produced by the oscillating airfoll motion is depicted in Figure 3.2.4. It is felt that the prediction of realistic wake geometries such as this will be an essential element in the eventual calculation of the unsteady airloads on airfoils in dynamic stall.

\subsection{Quasi-Steady, Separated Flows}

Figures 3.3.1 through 3.3.3 illustrate the comparison between computed and experimentally determined lift and drag curves for a NACA 0015 airfoil at three Reynolds numbers. The boundary layer separation points for these calculations were predicted on the basis of pressure distributions for steady, nonseparated flows at equivalent angles of attack. The potential and boundary layer calculations were not coupled in a step-by-step solution procedure. The resulting varlations of separation pulul lucation with respect to angle of attack are illustrated in Figure 3.3.4.

From the figures, it is noted that the lift curves are predicted with reasonable accuracy for the $\mathrm{R}_{e}=.665 * 10^{6}$ and $1.27 * 10^{6}$ cases and less well for the $R_{e}=.043 * 10^{6}$ case. The loss of accuracy there is most probably a consequence of the utilization of boundary layer correlations 


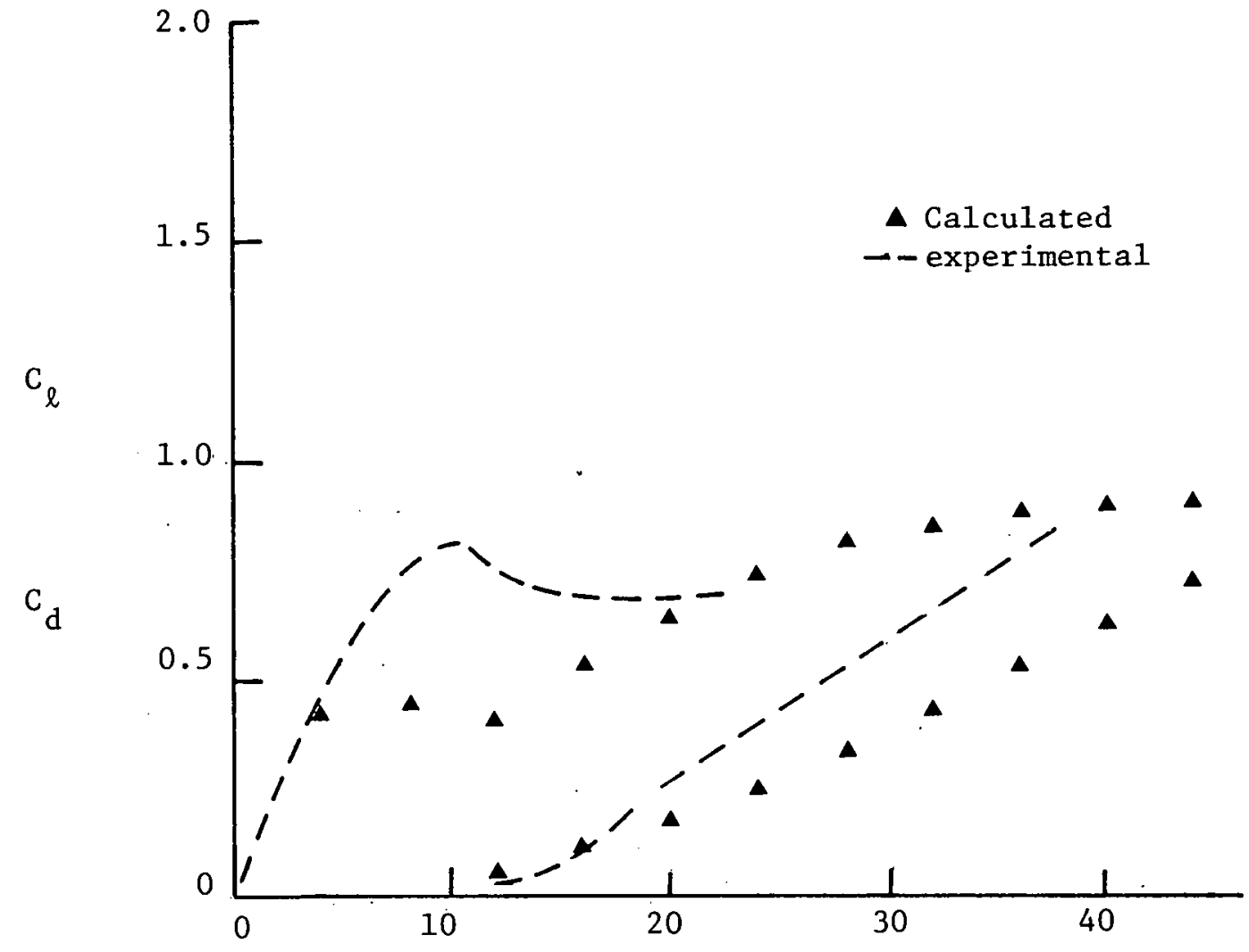

Figure 3.3.1 Variation of Lift and Drag Coefficients for a NACA 0015 at $\operatorname{Re}=42,900$ 


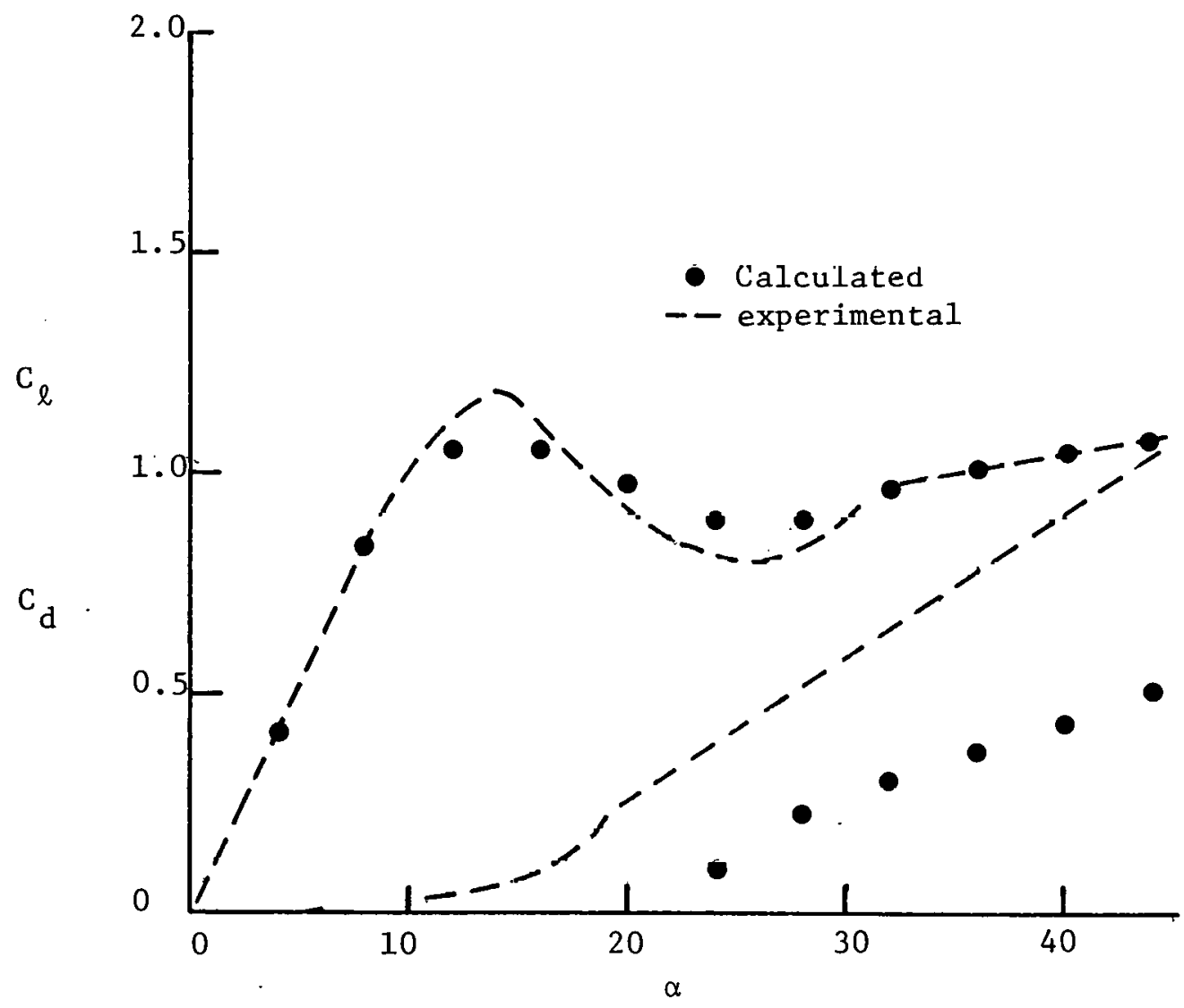

Figure 3.3.2 Variation of L1f 2 and Dray Cuefficients for a NACA 0015 at $\operatorname{Re}=655,000$ 


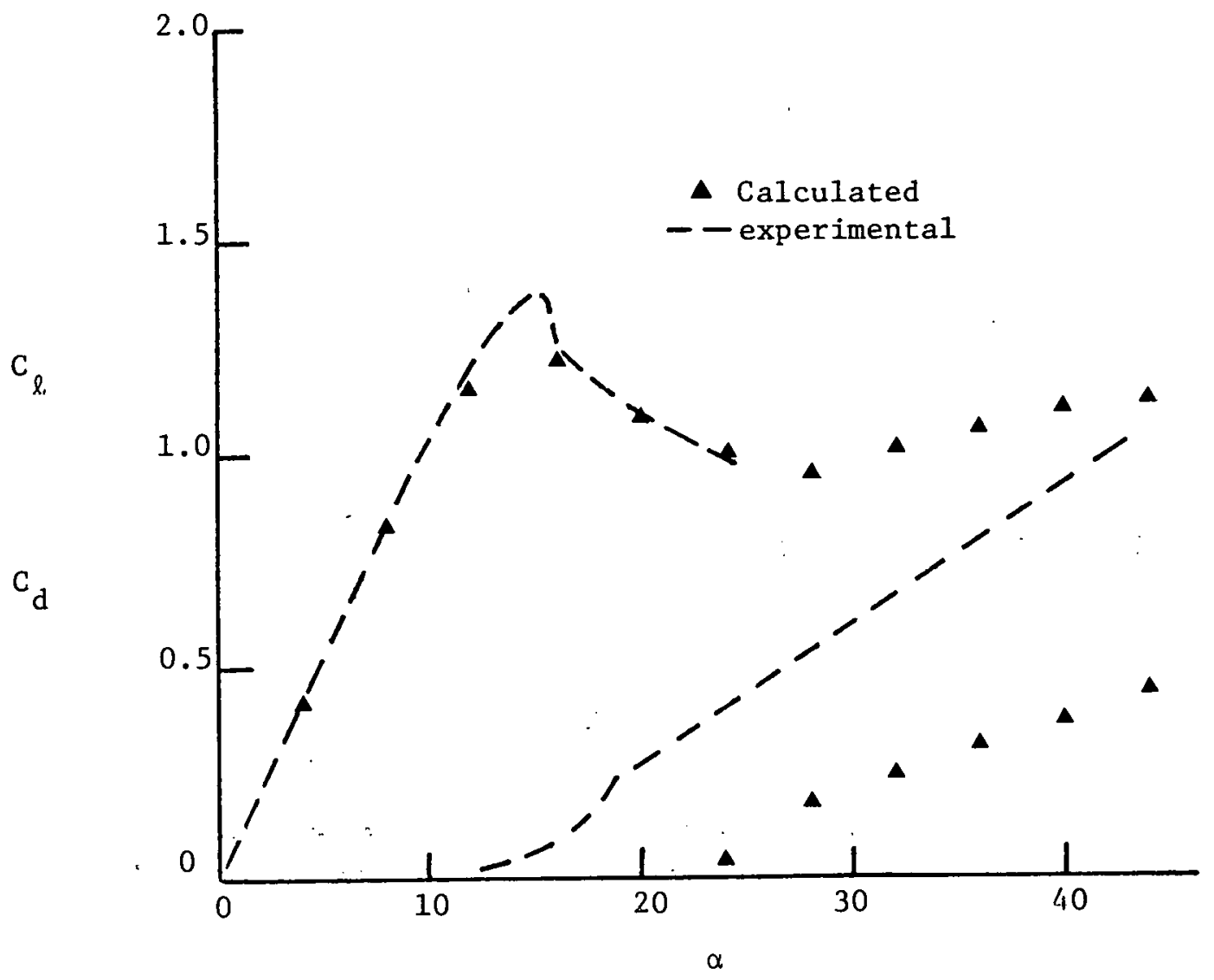

Figure 3.3.3 Variation of Lift and Drag Coefficients for a NACA 0015 Airfoil at $\operatorname{Re}=1,270,000$ 


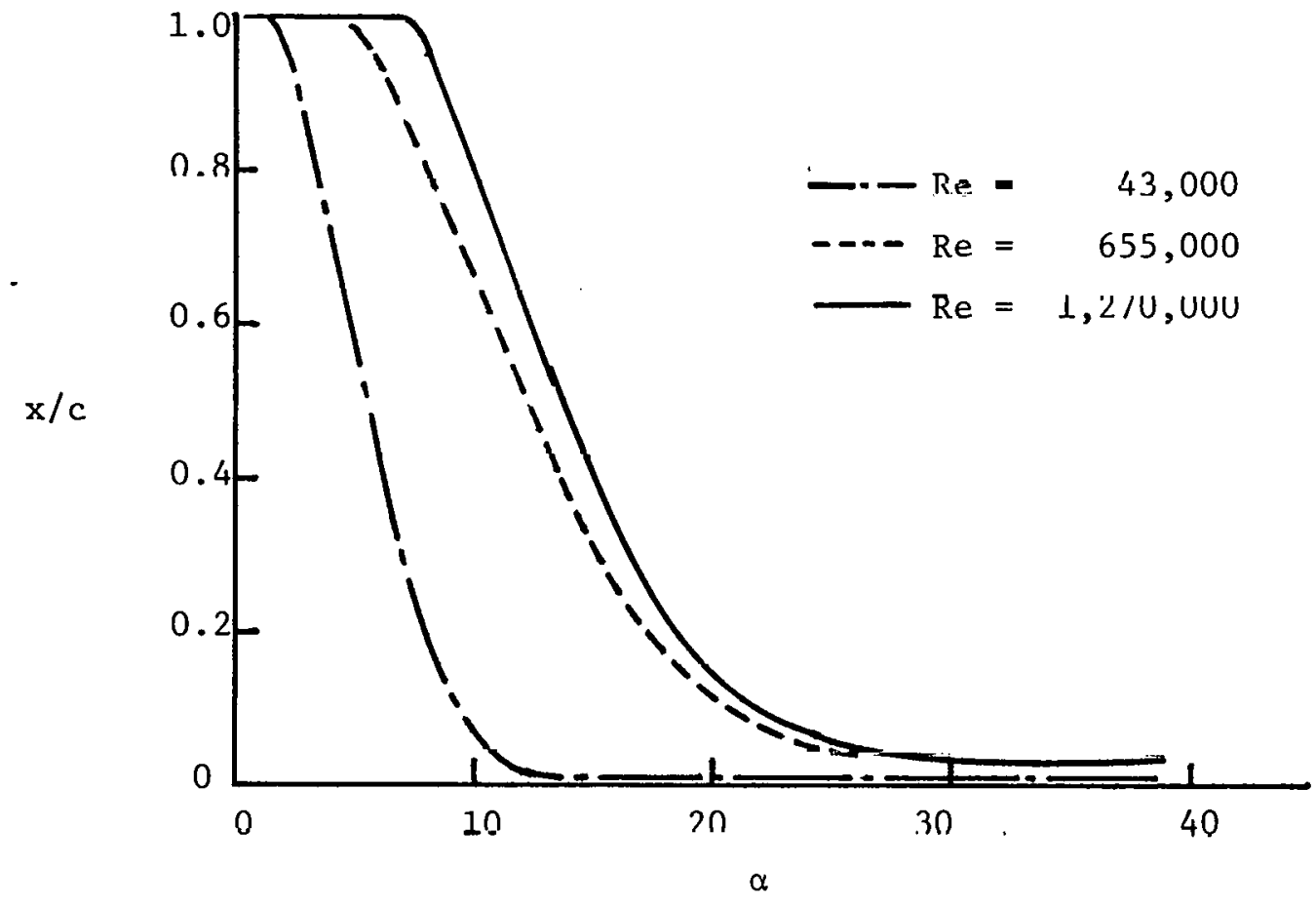

Figure 3.3.4 Variation of Boundary Layer Separation Point Location for a NACA 0015 Airfoil 
at $R_{e}$ below their range of applicability. It may also be noted that the drag is underestimated at all three Reynolds numbers.

As was described in the previous chapter, a reduction factor is utilized in determining the rate of vorticity shedding at the boundary layer separation point. Since the value of $\partial \phi / \sigma t$ and pressure in the separated region are directly related to the shedding rate, the reduction factor strongly affects the calculated airloads. The reduction factor for the 11lustrated calculations was chosen such that the best prediction of lift at post-stall angles of attack was achieved. The resulting value was 0.5 . Unfortunately, this reduction factor value did not yleld acceptable accuracy for the drag coefficients.

It is expected that there exists a unique combination of the reduction factor and separation point location that will yield satisfactory predictions of both lift and drag. Figure 3.3.5 1llustrates a slight modification of the separation point versus angle of attack relationship for the $R_{e}=.655 * 10^{6}$ case. When used with a reduction factor of 0.55 instead of 0.5 , the calculated lift and drag are significantly improved as illustrated in Figure 3.3.6. A modification of separation point location such as that In Figure 3.3 .5 is likely to occur when the potential and boundary layer routines are directly coupled. Figure 3.3.7 illustrates the calculated separated flow pressure distribution for $\alpha=24^{\circ}, \mathrm{R}_{e}=.655 * 10^{6}$. The difference between this distribution and the nonseparated distribution which was used in the boundary layer calculations is apparent.

In recent attempts to couple the viscid and inviscid calculations on a step-by-step basis, unrealistically erratic movement of the boundary layer separation point was predicted. This was a result of attempting to ut1lize 


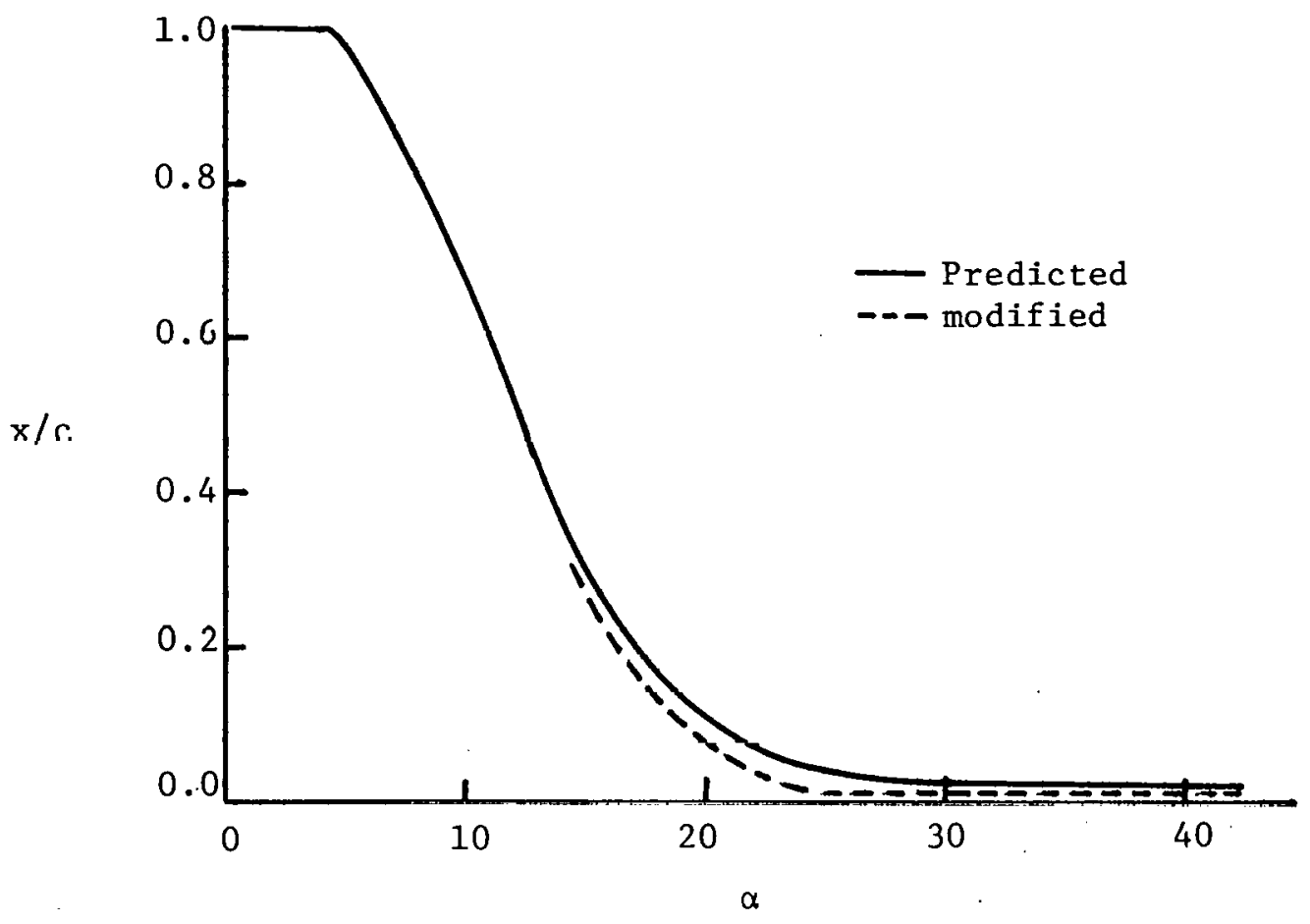

Figure 3.3.5 Alternate Variation of Boundary Iayer Separation Point Losation for a NACA (i)ili Airfnil. at $\mathrm{ke}=655,000$ 


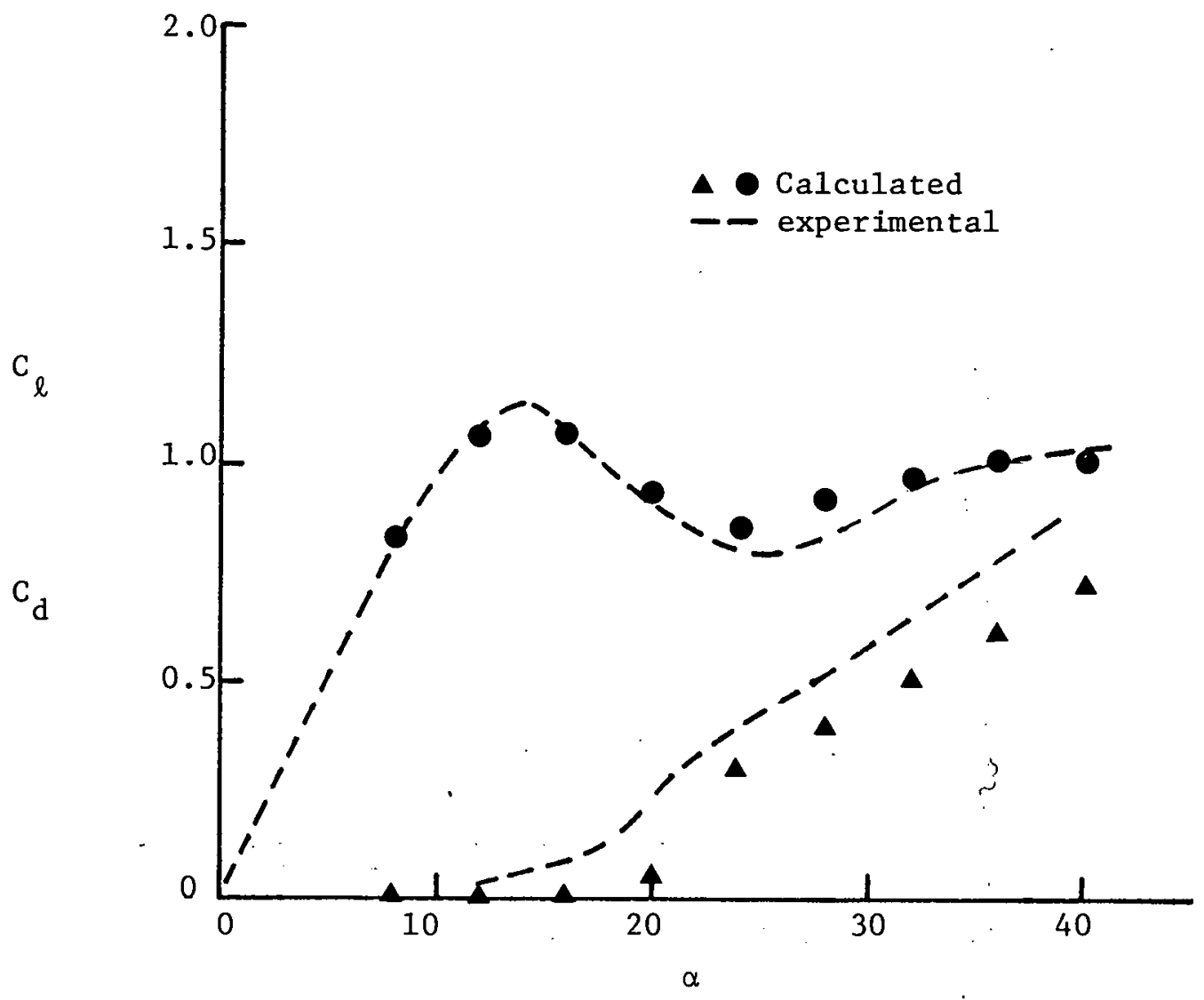

Figure 3.3.6 Variation of Lift and Drag Coefficients for a NACA 0015 Airfoil at $\operatorname{Re}=655,000$ 


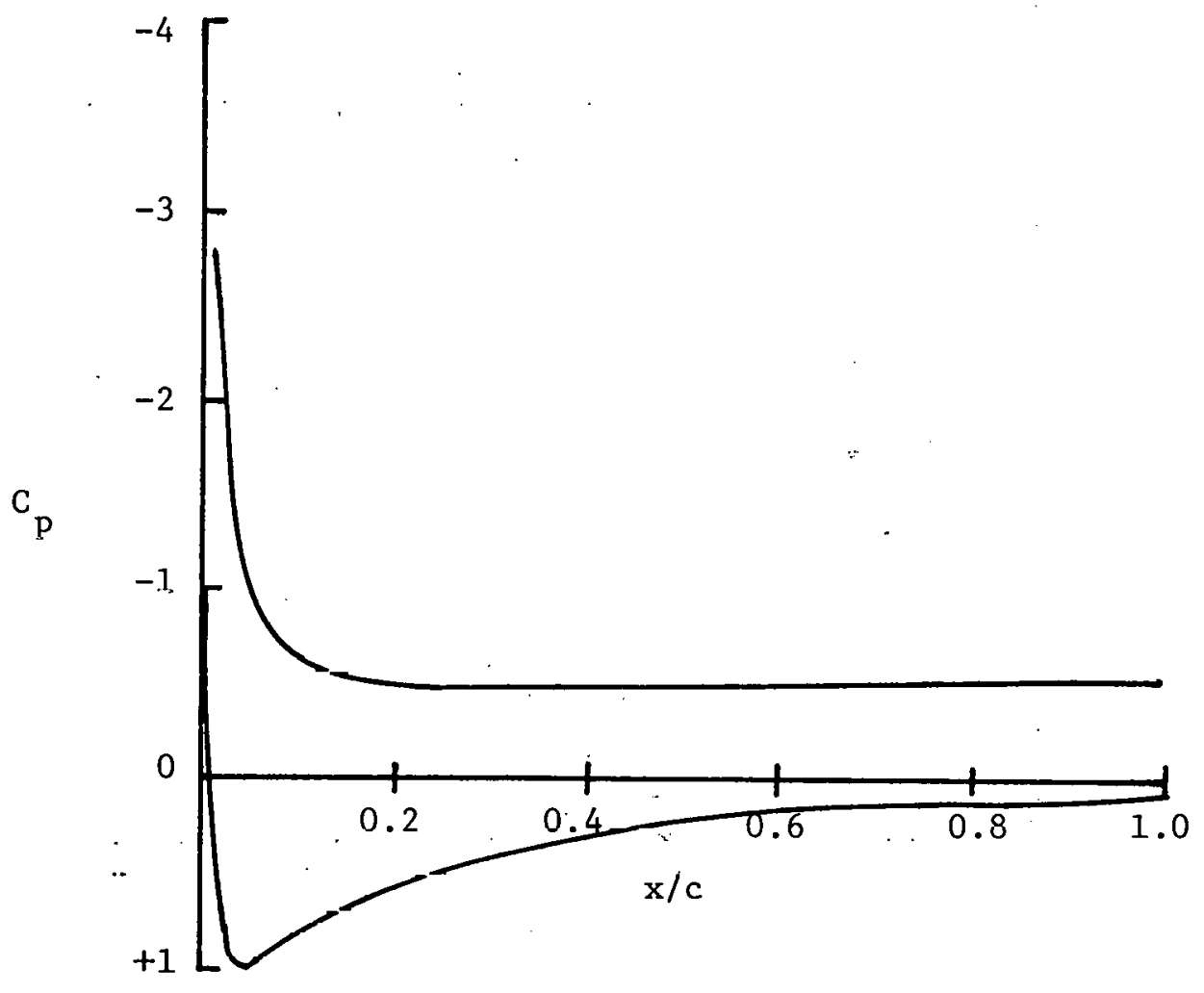

Figture 3.3.7 Calculated Pressure Distribution on a NACA nก15 Airfoil at $24^{\circ}$ angle of Attack. and $\operatorname{Re}=655,000$ 
an incomplete and inaccurate modeling of the viscid/inviscid interactions In the immediate vicinity of the separation point. In DYNA2, the separation process is represented by a surface of potential discontinuity emanating from the boundary layer separation point. This wake surface Influences. the pressure distribution and, hence, has a feedback effect on the location of the boundary layer separation point. Therefore, the precise manner with which the wake surface is generated has a dramatic effect on the boundary layer calculations and the airload predictions as a whole. Additional development of DYNA2 in this area is needed.

Figure 3.3.8 illustrates a calculated wake geometry behind an airfoll with boundary layer separation. The geometry is qualitatively as would be expected. 


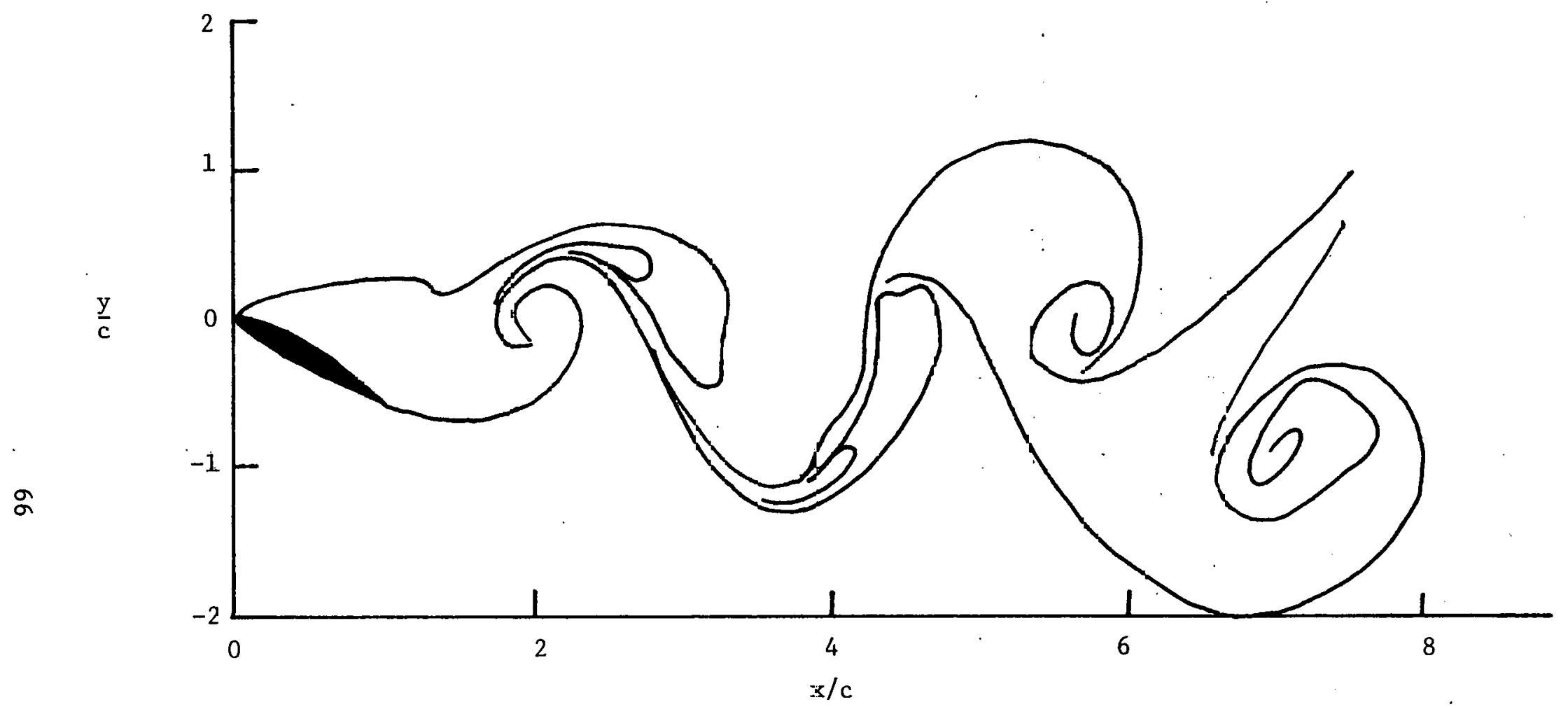

Figure 3.3.8 Computed Wake Geometry Behind a NACA 0015 Airfoil at an Angle of Attaci. Equal to $30^{\circ}$ 


\section{DYNAMIC STALL EXPERIMENT}

\subsection{Objectives}

The original motivation for pursuing an experimental investigation in parallel with the analytical study was to provide a means of verifying the model DYNA2 which is described in the previous sections. Unfortunately the present status of DYNA2 does not allow direct comparison since DYNA2 has not progressed to the point where the Darrieus flight path can be simi1ated. Eventually, however, DYNA2 will yleld predictions of Darrieus turbine aerodynamic characteristics for which there are currently no avallable experimental counterparts. While it is true that there is a large amount of avallable experimental data for oscillating airfolls, there is also a need for unsteady aerodynamic data specific to Darrieus turbines. The experimental work described in the following sections represents a significant beginning effort almed at alleviating that need.

The experiments which were conducted were designed to determine the characteristics of the unsteady blade loadings and dynamic stall phenomena as they occur on Darrieus turbines. Simllar experiments on afrfoils oscillating in pitch have revealed that the oscillation amplitude and reduced frequency of the oscillations are key parameters in determining the significance of the unsteady aerodynamic effects (see Martin, et al., 1974 and Cebecci and Smith, 1974). In the present work the tip-to-wind speed ratio is indicative of the oscillation amplitude and will be varied over a limited range. The chord to radius ratio is indicative of the oscillation frequency and will be fixed a a value of $C / R=0.25$. 


\subsection{General Test Setup}

The general test setup is described by Strickland (1980) in detall and will be described only briefly herein. In general, a straight-one-bladed rotor with a NACA 0015 airfoil was built and operated in a water tow tank with a depth of 1.25 meters, a width of 5 meters, and a length of 10 meters. Some testing was also done using a SANDIA 0015/47 airfoil. The rotor blades extended to within 15 centimeters of the tank bottom. This simple rotor appeared to be adequate for validating the major features of the analytical model. The use of water as a working fluid greatly facilitates the ability to make relatively low frequency measurements while working at appropriate blade Reynolds numbers. In addition, blade forces and pressures are more eas1ly measured. An airfoll chord length of 15.24 $\mathrm{cm}$ and a rotor tip speed of $45.7 \mathrm{~cm} / \mathrm{sec}$ were chosen to yield a blade Reynolds number of 67,000 . Three towing speeds of $18.3 \mathrm{~cm} / \mathrm{sec}, 9.1 \mathrm{~cm} / \mathrm{sec}$, and $6.1 \mathrm{~cm} / \mathrm{sec}$ were chosen to yleld tip-to-wind speed ratios of $2.5,5.0$, and 7.5 , respectively. The rotor diameter was chosen to be 1.22 meters, thus giving a chord to radius value $(C / R)$ of 0.25 . Blade attachment was at mid chord in all cases.

Data were acquired using the Mechanical Engineering Department HP9835A desktop computer coupled to a multichannel HP6940B analog to digftal converter and a HP7225A plotter. The system is capable of acquiring analog signals from an experiment at rates in excess of $1 \mathrm{KHz}$, which was quite adequate in light of the rotor rotational speed of $0.12 \mathrm{~Hz}$. The synchronization of the rotor position for various runs was extremely important. The rotor has a transducer (Waters Mfg. Analyzer APT 55) mounted on the main shaft, which allowed the rotor angular position to be monitored and recorded along with whatever other parameter was being measured. 
Calfbration and input data for each run were stored on magnetic tape cartridges which are compatible with the HP9835A. Each cartridge is capable of storing $256 \mathrm{~K}$ Bytes of information or about $128 \mathrm{~K}$ data points on 42 files.

\subsection{Pressure Measurements}

Pressure measurements were made on both sides of the NACA 0015 airfoll at five locations. The pressure ports were located at $x / c$ values of $0.017,0.042,0.100,0.360$, and 0.810 at a unfform depth of about $30 \mathrm{~cm}$ below the water surface. The pressure ports were connected to diaphram pressure transducers (Validyne DP45-16) via copper tubing which was Inserted through the hollow cores of the blade. The diaphram pressure transducers were connected to a Validyne demodulator unft (CD 18) which converted the pressure signal into an analog voltage sultable for introduction into the HP data acquisition system. A schematic of the arrangement is shown in Fig. 4.3.1.

The pressure measurements were made on one side of the airfoll and then the other. The inner side denotes the surface closest to the axis of rotation while the outer side denotes the side farthest from the axis of rotation. At least five repetitive runs were made to check the repeatability of the data.

Typical pressure data are shown in Fig. 4.3.2 for the inner and outer surfaces at $x / c=0.10$ as a function of rotor position. Multiples of 360 degrees correspond to the nose of the foll pointing directly into the flow. These data are the average of five runs and differ very little from each Individual run. 


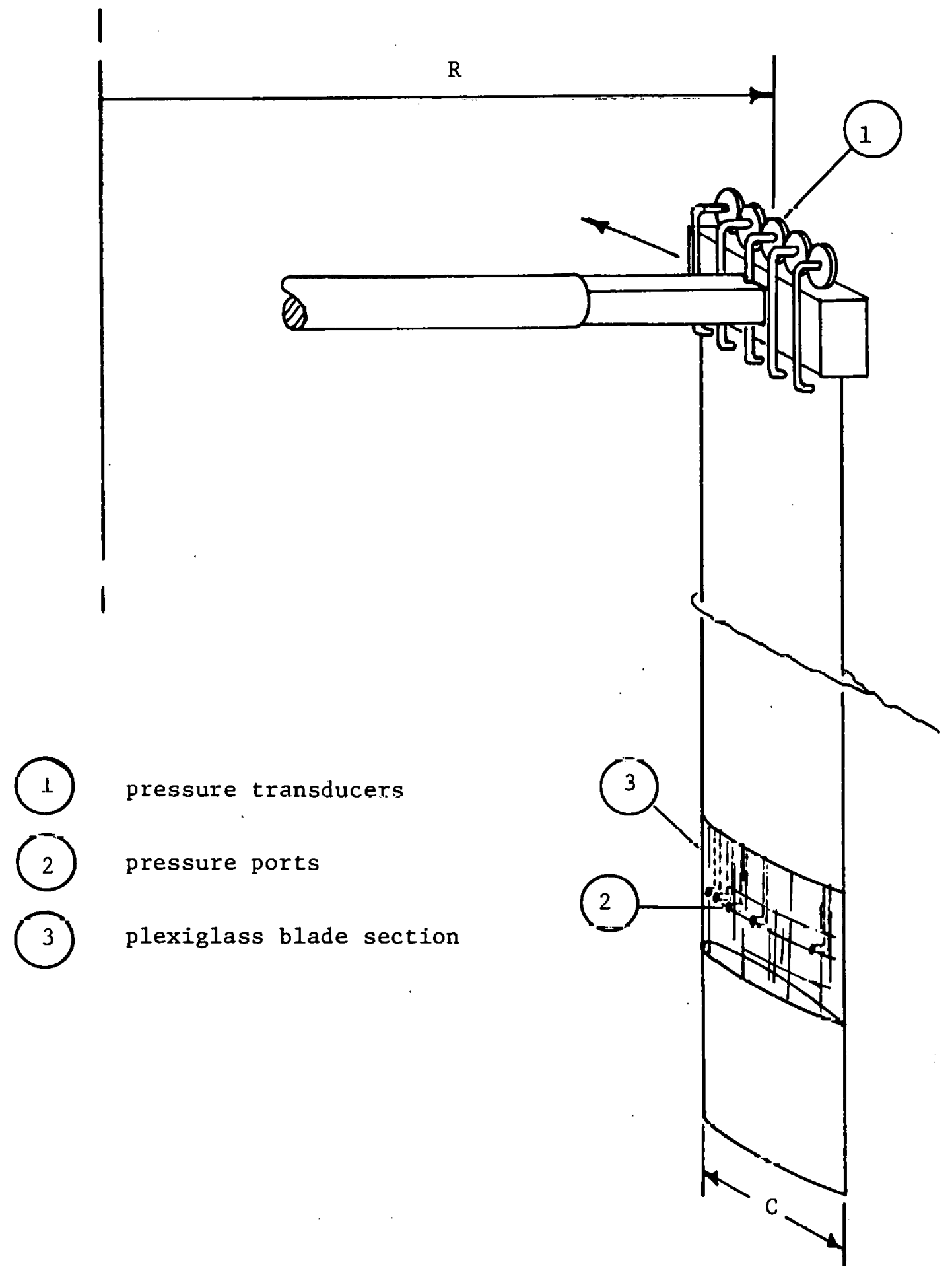

Fig. 4.3.1 Arrangement of Pressure Taps and Transducers 

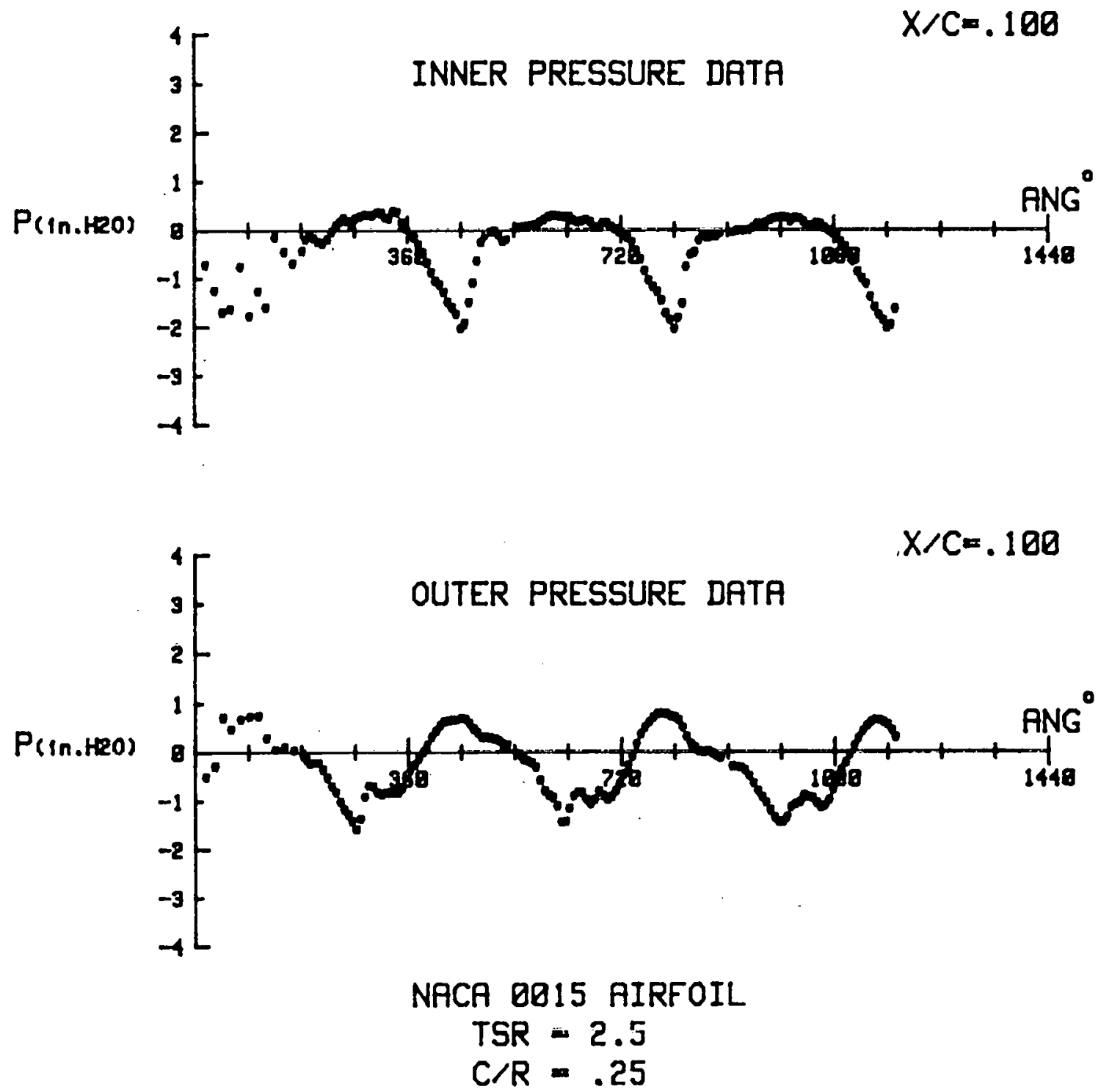
Fig. 4.3.2 Typical Pressure Data as a Function of Rotor Blade
Azimuthal Angle 
Typical pressure coefficient distributions for the airfoll at two different rotor angles are shown plotted in Fig. 4.3.3. The pressure coefficient in this case is defined by

where

$$
C_{p}=\frac{P-P_{\infty}}{1 / 2 \rho U_{b}^{2}}
$$

$$
\overrightarrow{\mathrm{U}}_{\mathrm{b}}=\overrightarrow{\mathrm{U}}_{\mathrm{T}}+\overrightarrow{\mathrm{U}}_{\infty}
$$

Here the velocities $U_{T}$ and $U_{\infty}$ are the blade tangential speed and the carriage speed (wind speed far from the rotor), respectively. The pressure $P_{\infty}$ is the static pressure at the depth below the surface corresponding to the pressure taps. A set of $C_{p}$ curves taken during the second revolution are given in Appendix E for tip-to-wind speed ratios of 2.5, 5.1, and 7.6.

The normal and tangential forces can be obtained by integrating the $C_{p}$ curves. Since only ten data points are available, the results may contain a reasonable amount of error. The integration was carried out using a second order polynomial fit to three data points in the subregion of integration. The results for the normal and tangential forces are given in Fig. 4.3.4 and Fig. 4.3.5 respectively. The nondimensional forces $\mathrm{F}_{\mathrm{n}}^{+}$and $\mathrm{F}_{\mathrm{t}}^{+}$ are defined by

$$
\begin{aligned}
& \mathrm{F}_{\mathrm{n}}^{+}=\frac{\mathrm{F}_{\mathrm{n}}^{\prime}}{1 / 2 \rho \mathrm{CU}_{\infty}^{2}} \\
& \mathrm{~F}_{\mathrm{t}}^{+}=\frac{\vec{T}_{\mathrm{t}}^{\prime}}{1 / 2 \rho \mathrm{CU}_{\infty}^{2}}
\end{aligned}
$$

where $F_{n}^{\prime}$ and $F_{t}^{\prime}$ are the normal and tangential forces per untt blade length. 


\subsection{Strain Gage Measurements}

Measurements of normal and tangential forces using strain gage instrumentation were performed for three tip-to-windspeed ratios of $2.5,5.1$, and 7.6. Five repetitive runs were made to determine the repeatability of the data. Tests were performed on both the NACA 0015 and the Sandia $0015 / 47$.

The experimental arrangement for obtaining strain gauge data is shown 1n Fig. 4.4.1. As indicated in Fig. 4.4.1 the two forces were measured using strain gages located on a support mounted at the mid chord. Each bridge was arranged so that it was only sensitive to the desired force.

The instrumentation consisted of elght 350 ohm strain gages making up the two Wheatstone bridge configurations, a 15 vdc Calex power supply, a Calex model 176 amplifier, and a Krohnite model 3343 low pass filter. A 15 vdc signal was applied across the bridge. As the bridge was strained the voltage caused by the unbalanced bridge was measured. This output voltage was amplified approximately 1000 times to increase the signal level into the desired voltage range. The output signal from the bridge was passed through a filter to low pass the signal below a cutoff frequency of $0.6 \mathrm{~Hz}$. This was necessary in order to eliminate extraneous mechanical notse at about $2 \mathrm{~Hz}$. Finally, the signal was introduced into the data acquisition system.

The output voltages from the strain gage bridges $E_{1}$ and $E_{2}$ are related to the blade forces by

$$
\begin{aligned}
& F_{n}=C_{1} E_{1} \\
& F_{t}=C_{2} E_{2}
\end{aligned}
$$

where $C_{1}$ and $C_{2}$ are calibration constants. It should be noted that $E_{1}$ includes the effect of the centrifugal force given by

$$
\mathrm{F}_{\mathrm{c}}=\mathrm{mR} \omega^{2}
$$



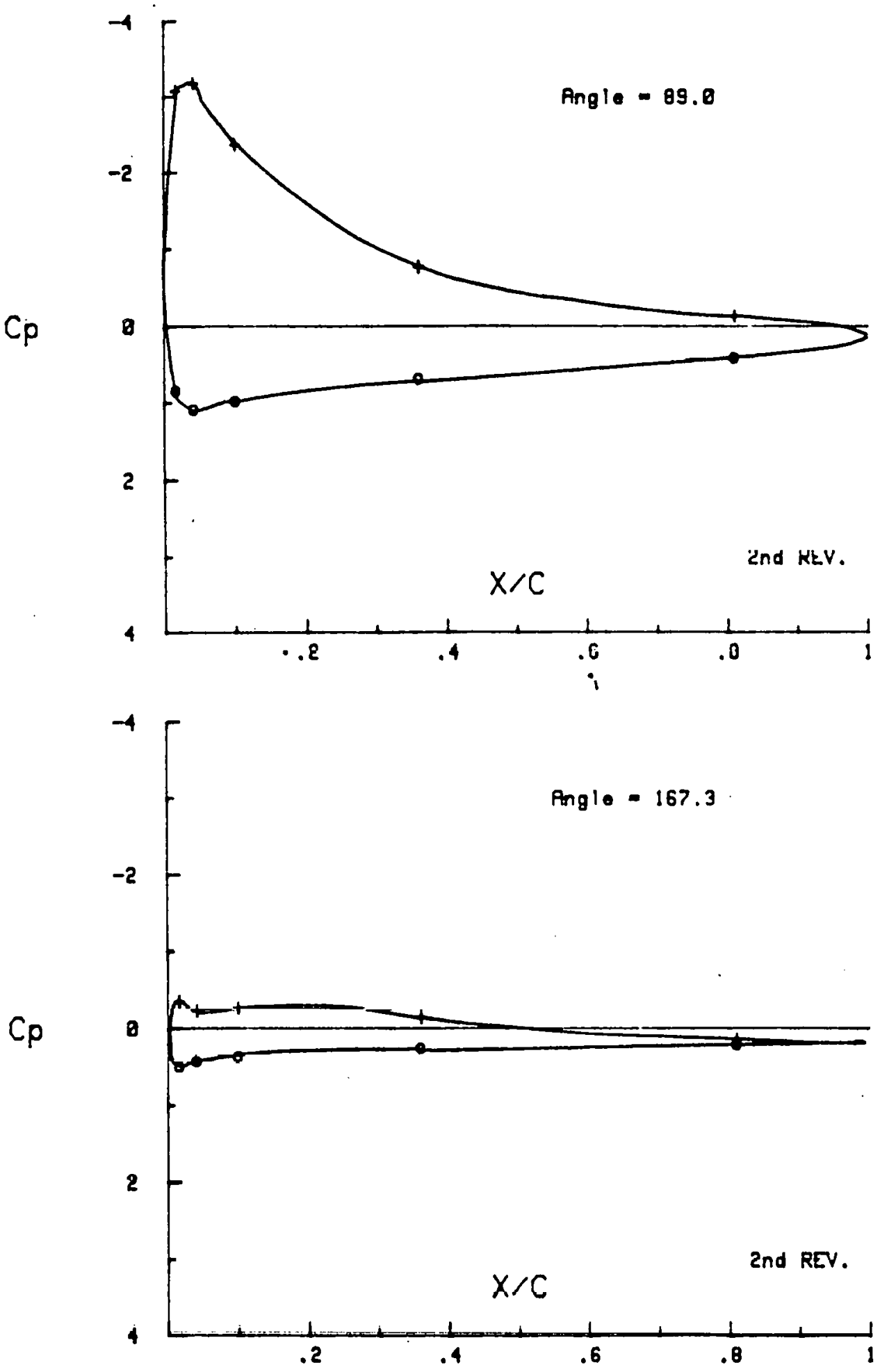

Fig. 4.3.3 Typical Pressure Coefficients

(TSR $=2.5, C / R=0.25, \mathrm{NB}=1$, NACA0015, Outer Surface 0, Inner Surface + ) 

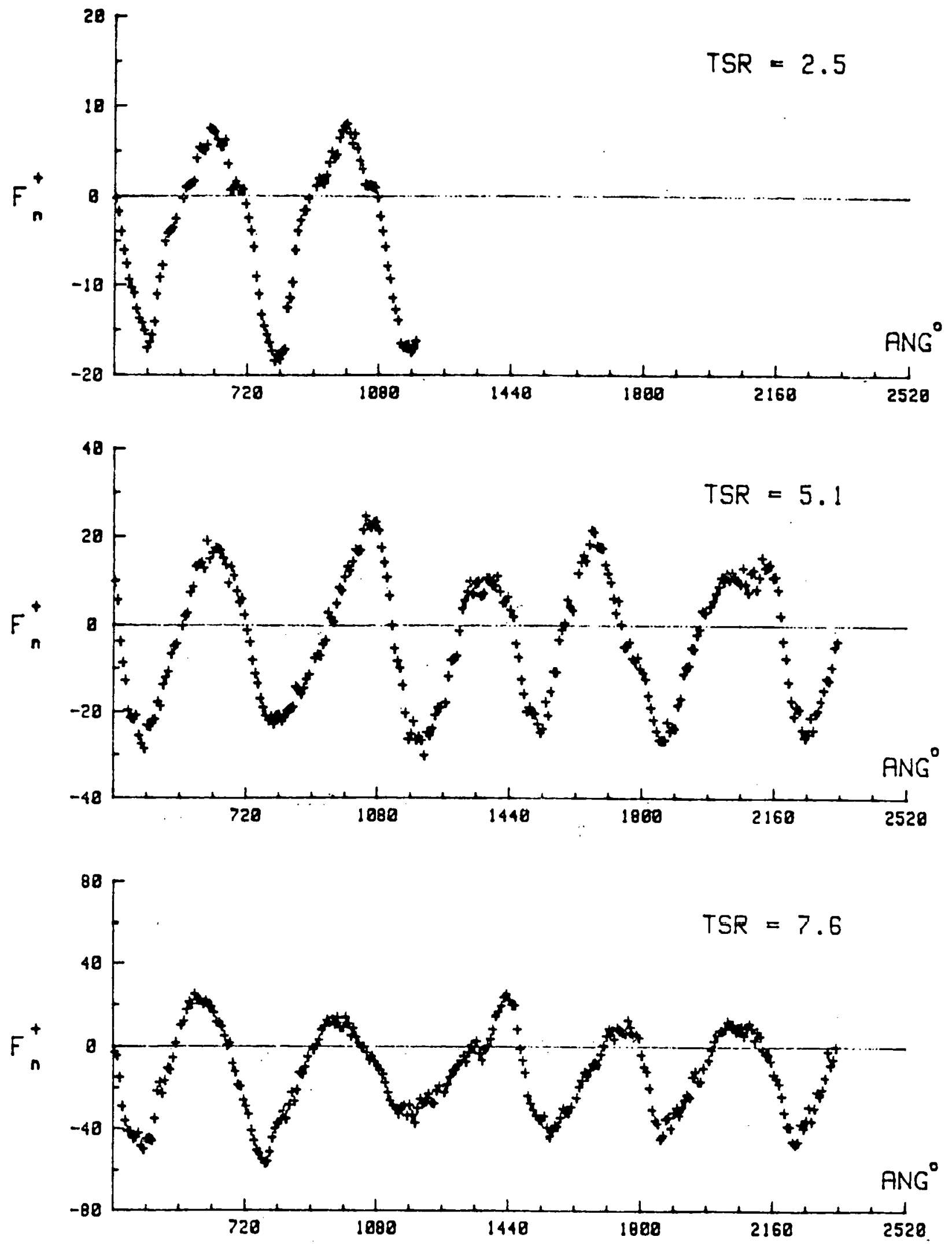

Fig. 4.3.4 Normal Pressure Force Data for NACA 0015 Rotor 

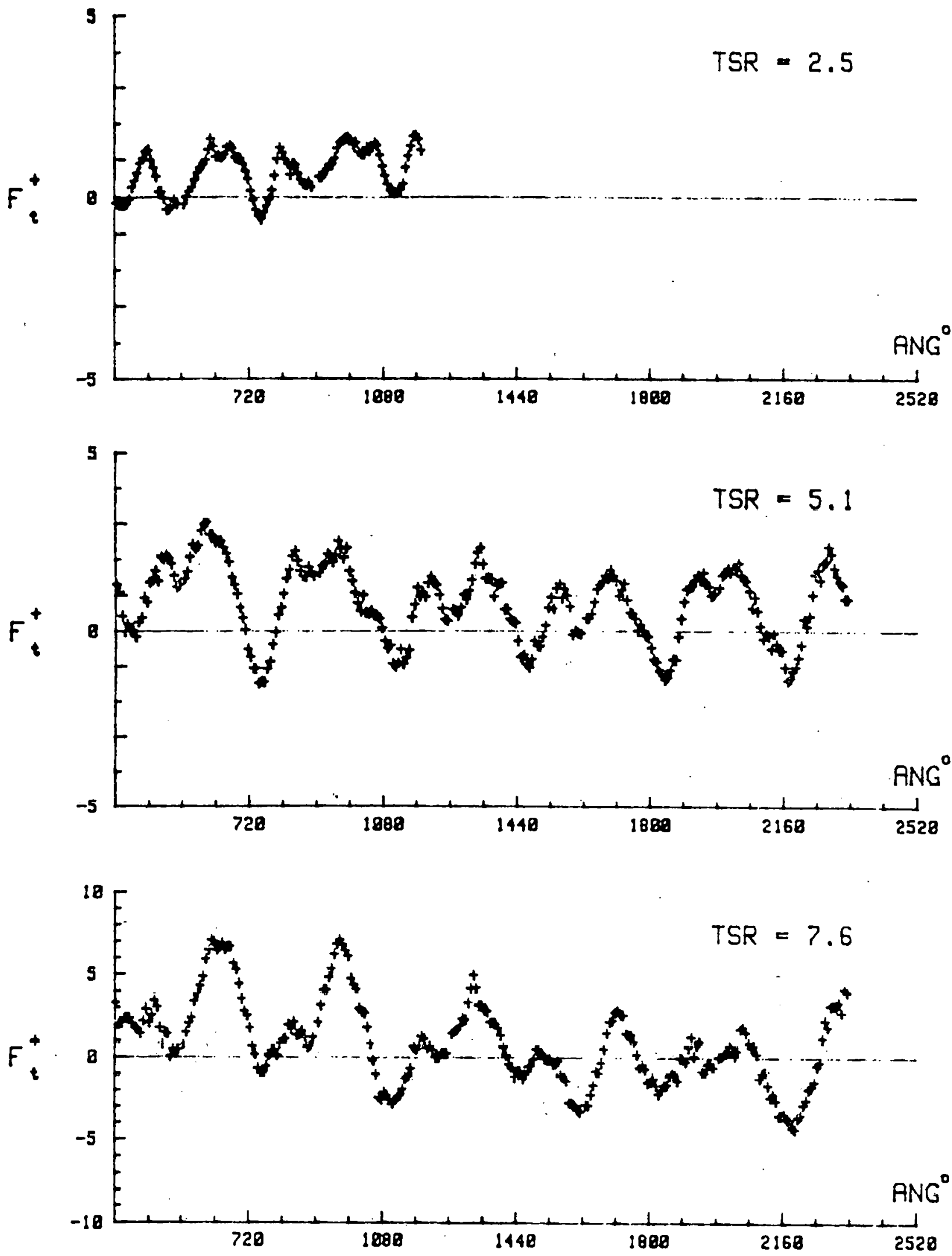

Fig. 4.3.5 Tangential Pressure Force Data for NACA 0015 Rotor 


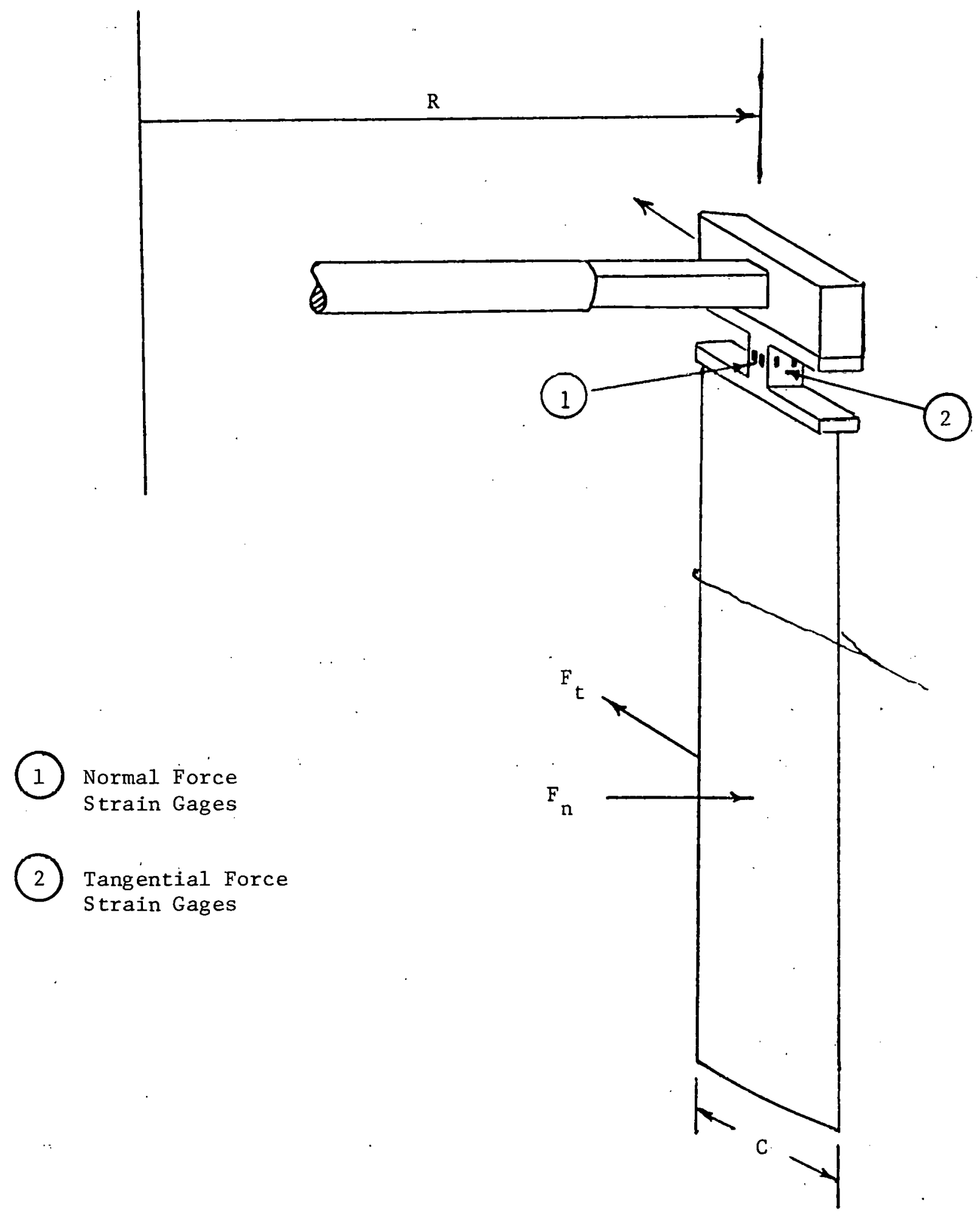

Fig. 4.4.1 Strain Gage Instrumentation 
where $\mathrm{m}$ is the mass of the blade. While this effect is relatively small it was nevertheless subtracted from the equation for $F_{n} \cdot$ All cases were run at the same angular speed (tip speed) and thus $F_{c}$ was constant for all cases. Additionally, the strain gage data were corrected for finite aspect ratio effects; 1.e. Induced drag and 11ft. The effective aspect ratio of the blade was approximately 10.5 and therefore these corrections tend to be small. Details of this correction are given by Graham (1982).

Strain gauge data for normal and tangential forces on the NACA 0015 rotor are given In Figures 4.4 .2 and 4.4.3. Data for the Sandia 0015/47 rotor are shown superimposed on the NACA 0015 data in Figures 4.4 .4 and 4.4.5.

\subsection{Discussion of Results}

The experimental results which were obtained as a part of this study cannot be compared to results from the DYNA2 mode1 at the present time. Some comparisons between the VDART2 model by Strickland (1981(a) and $1981(b))$ can be made along with comparisons between the integrated pressure force data and the strain gage data. In addition, the instantaneous pressure data can be used to yleld some information on the progression of stall on the airfoll.

\section{5 .1 Blade Forces}

Typical non-dimensional blade forces are shown in Figures $4.5 .1,4.5 .2$ and 4.5.3. These forces are defined by Equations 4.3.2. A positive normal force acts radially outward while a positive tangential force acts in the direction of motion of the alrfoll. Blade forces obtained from integrated pressure measurements, strain gage measurements, and analysis are plotted in these figures. 

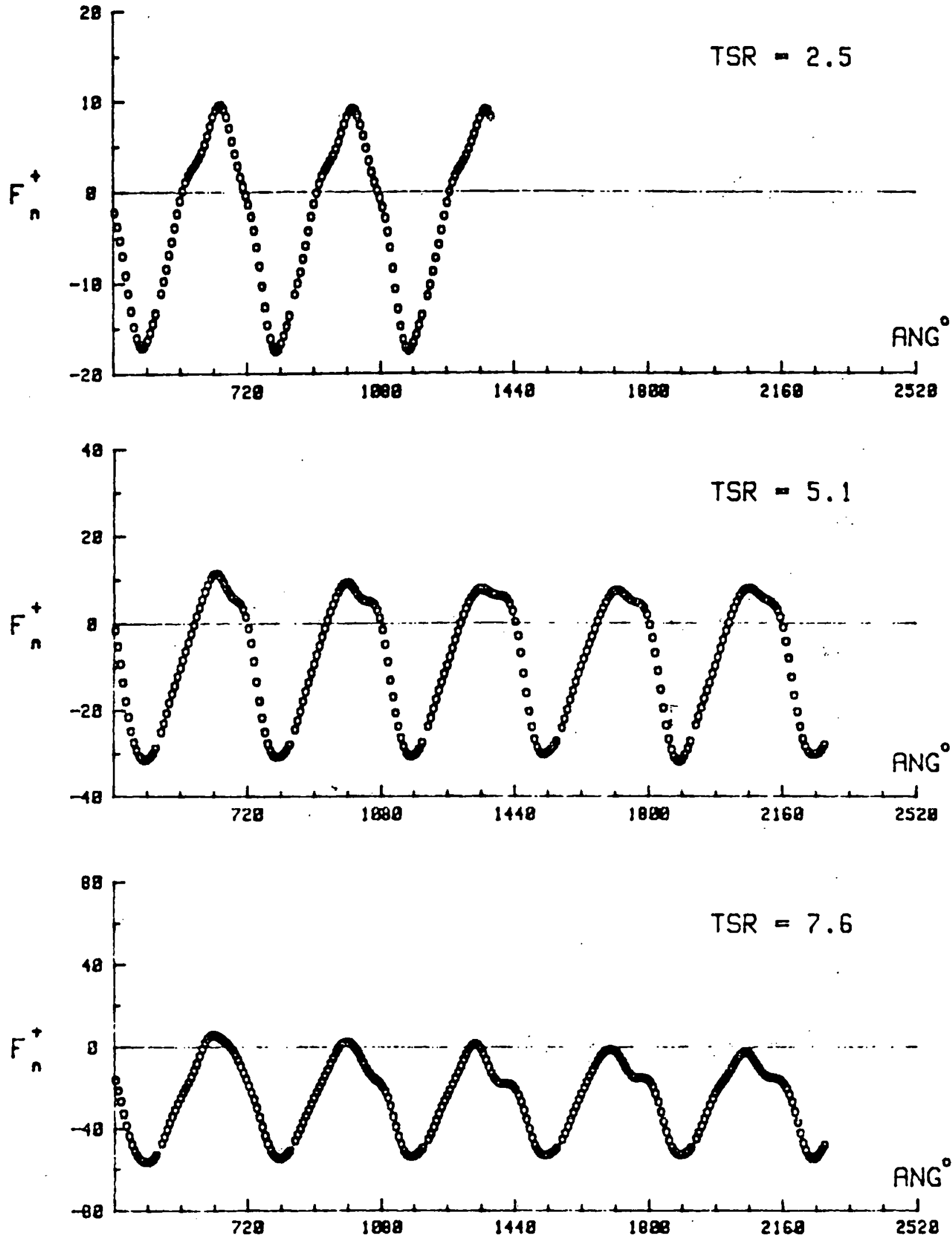

Fig. 4.4.2 Normal Strain Gage Force Data from NACA 0015 Rotor 

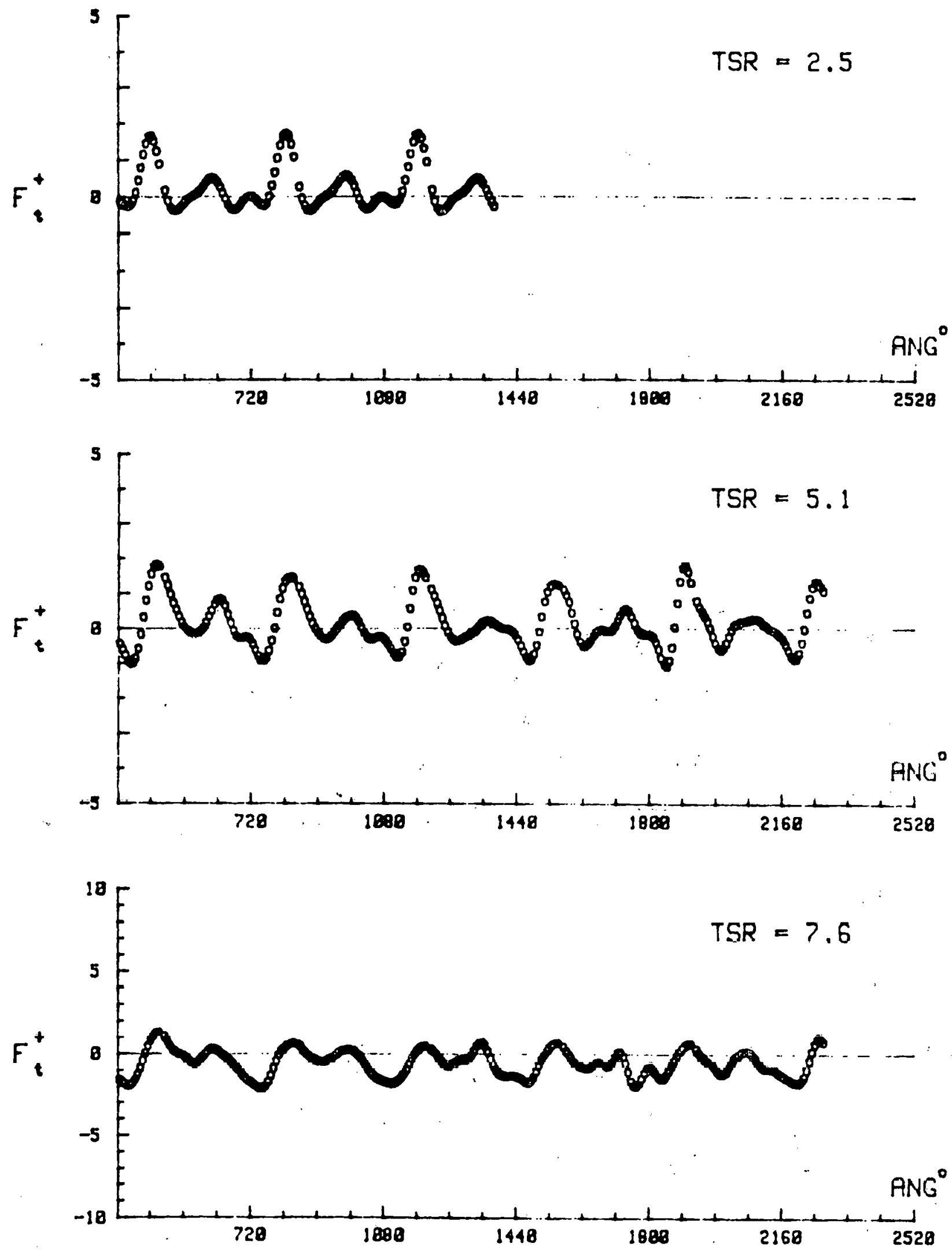

Fig. 4.4.3 Tangential Strain Gage Data from NACA 0015 Rotor 

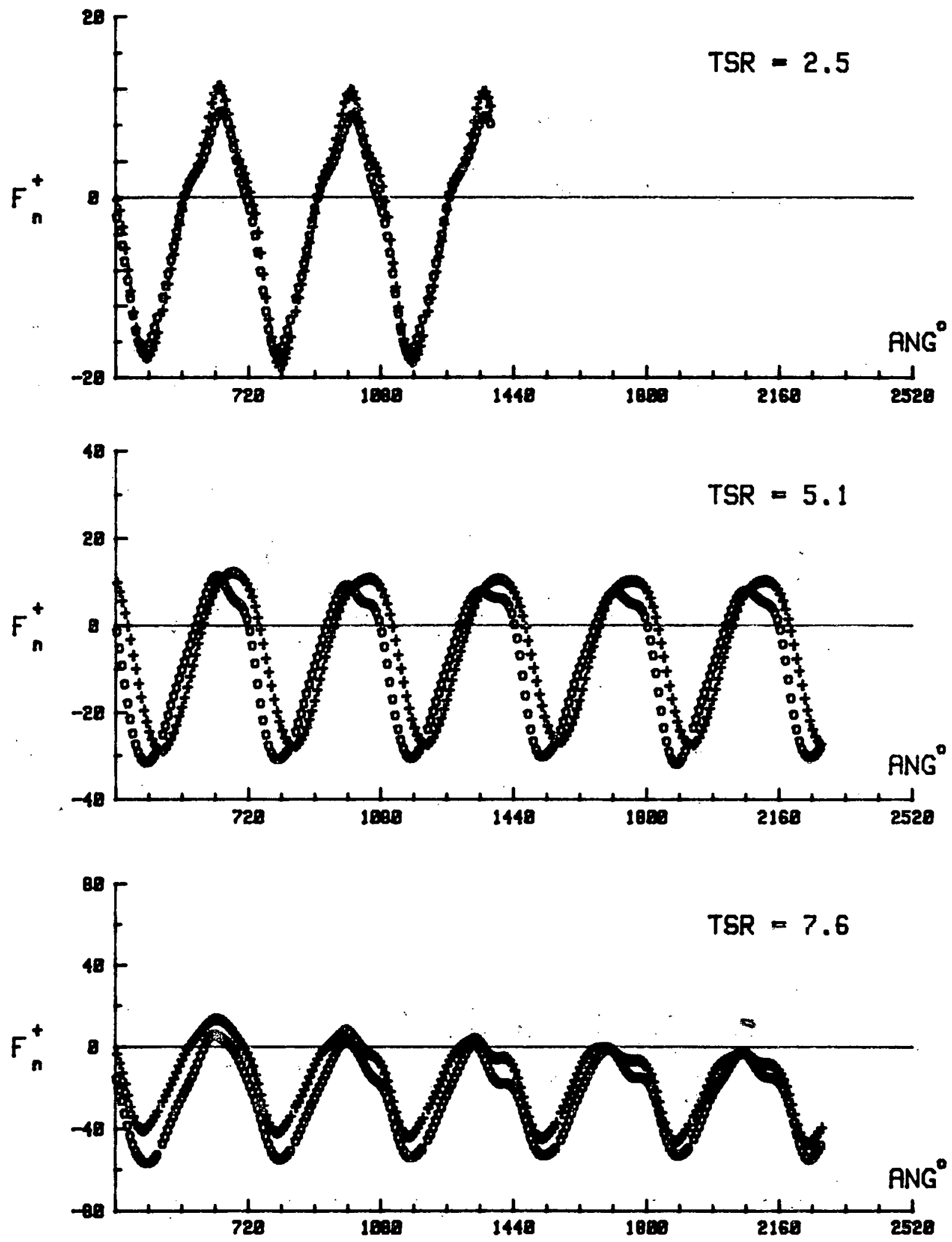

F'i.g. 4.4.4 NACA 0015 and SANDIA 0015/47 strain Gage Normal Force Data 

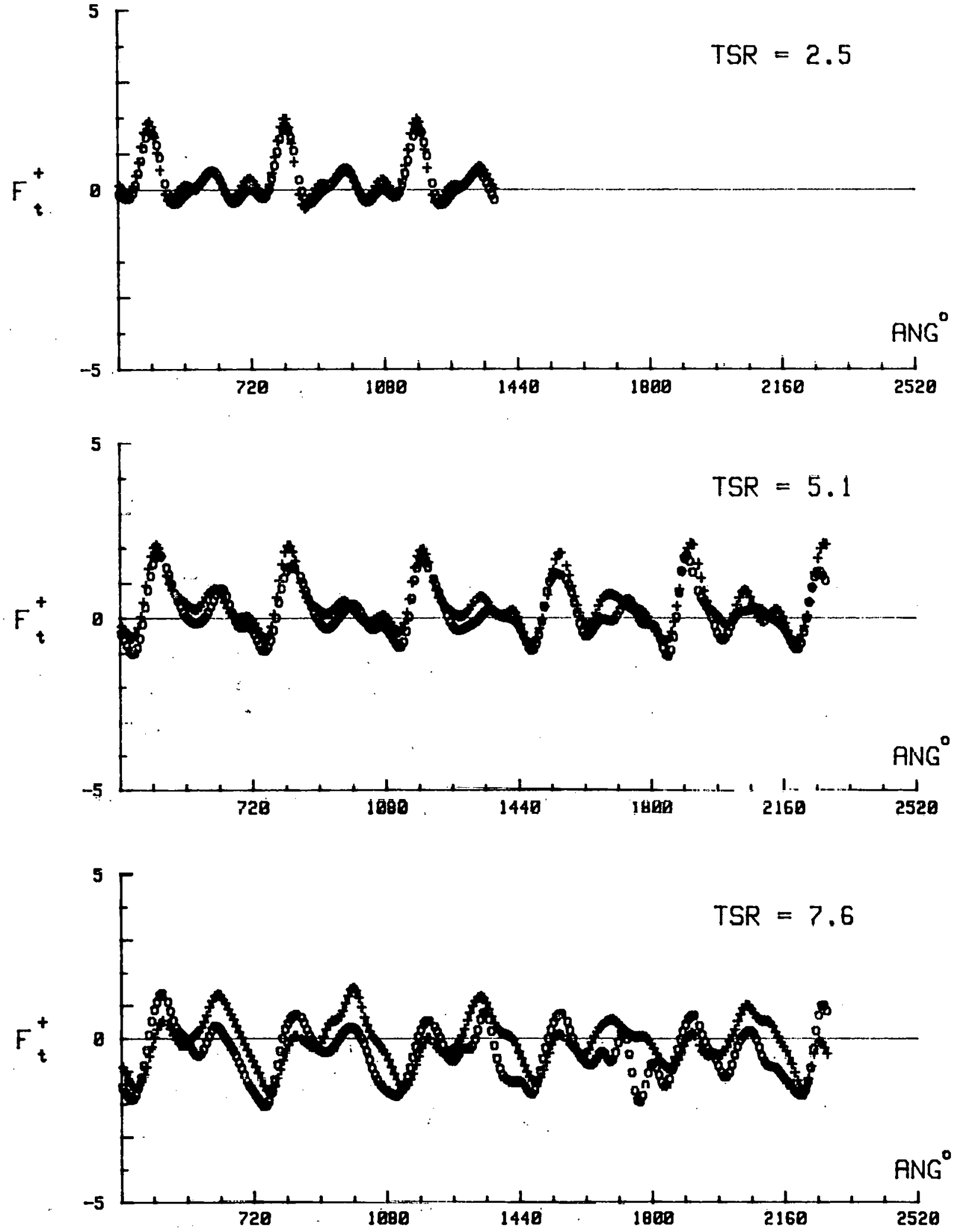

Fig. 4.4.5 NACA 0015 and SANDIA 0015/47 Strain Gage Tangential Force Data 

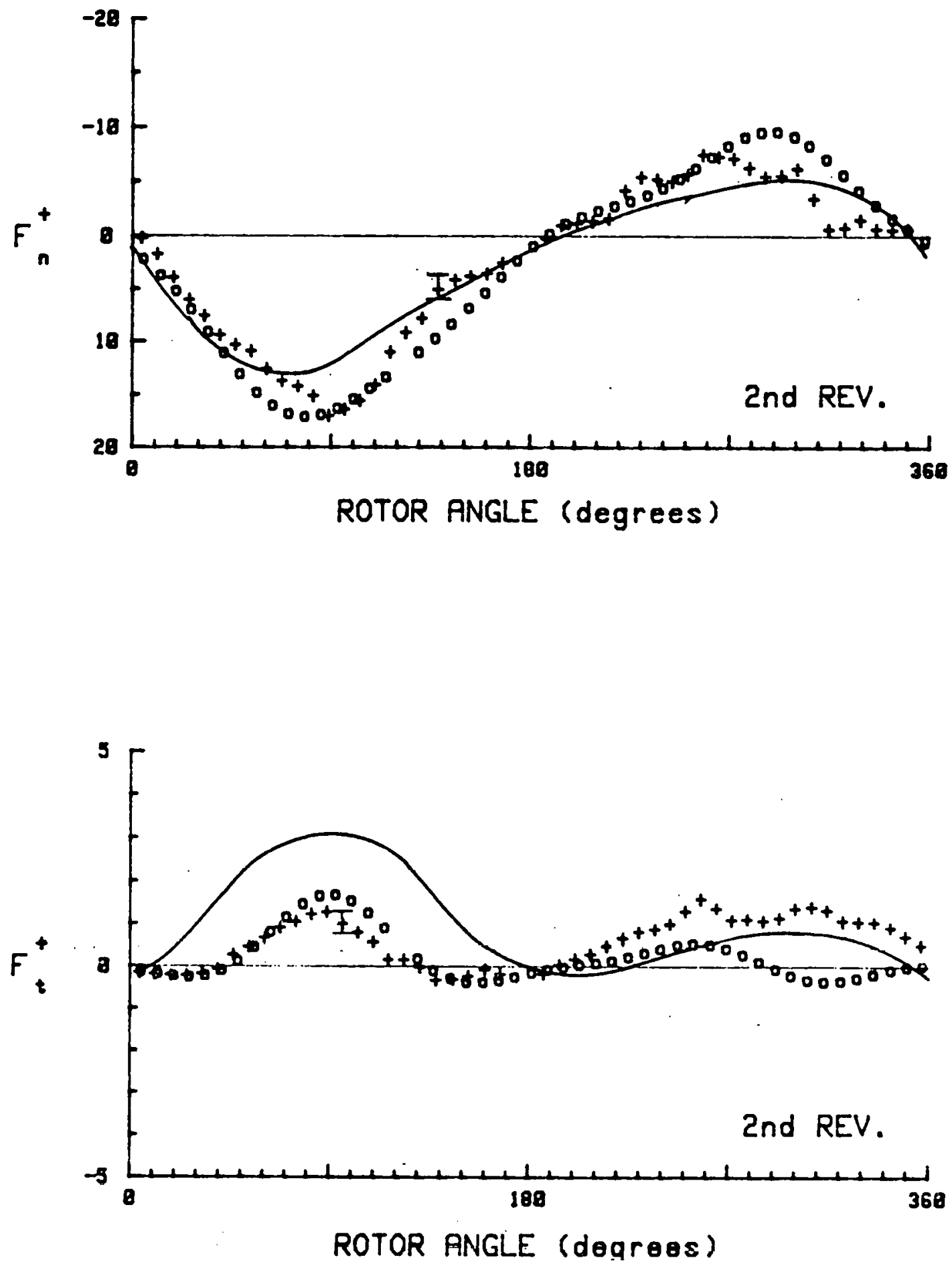

Fig. 4.5.1 Non-Dimensional. Blade Forces

(TSR $=2.5, C / R=0.25, N B=1$, Pressure Data + , Strain Gage 0, VDART2 -) 

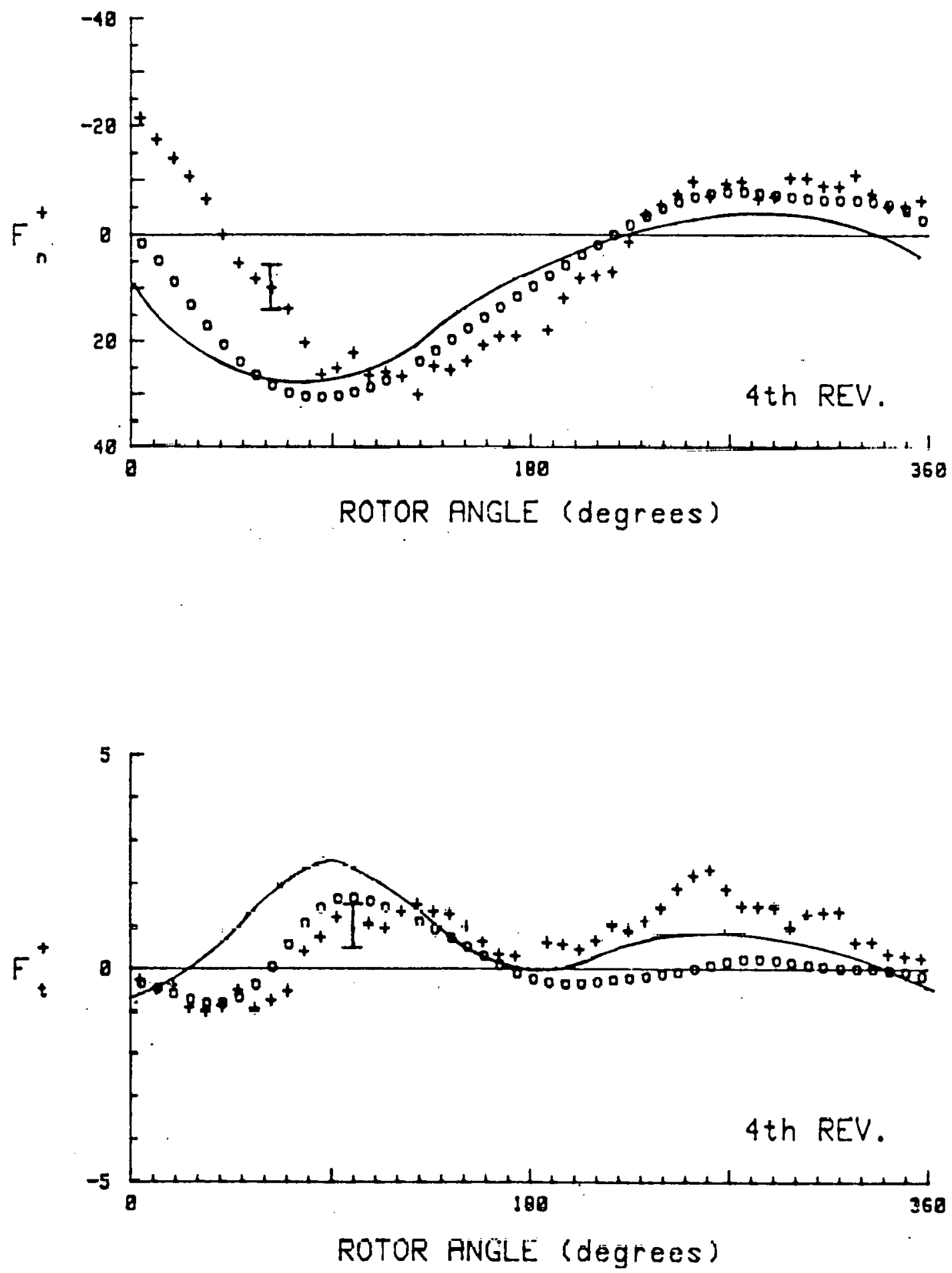

Fig. 4.5.2 Non-Dimenstonal Blade Forces

$(\mathrm{TSR}=5.1, \mathrm{C} / \mathrm{R}=0.25, \mathrm{NB}=1$, Pressure Data + , Strain Gage 0, VDART2 -) 

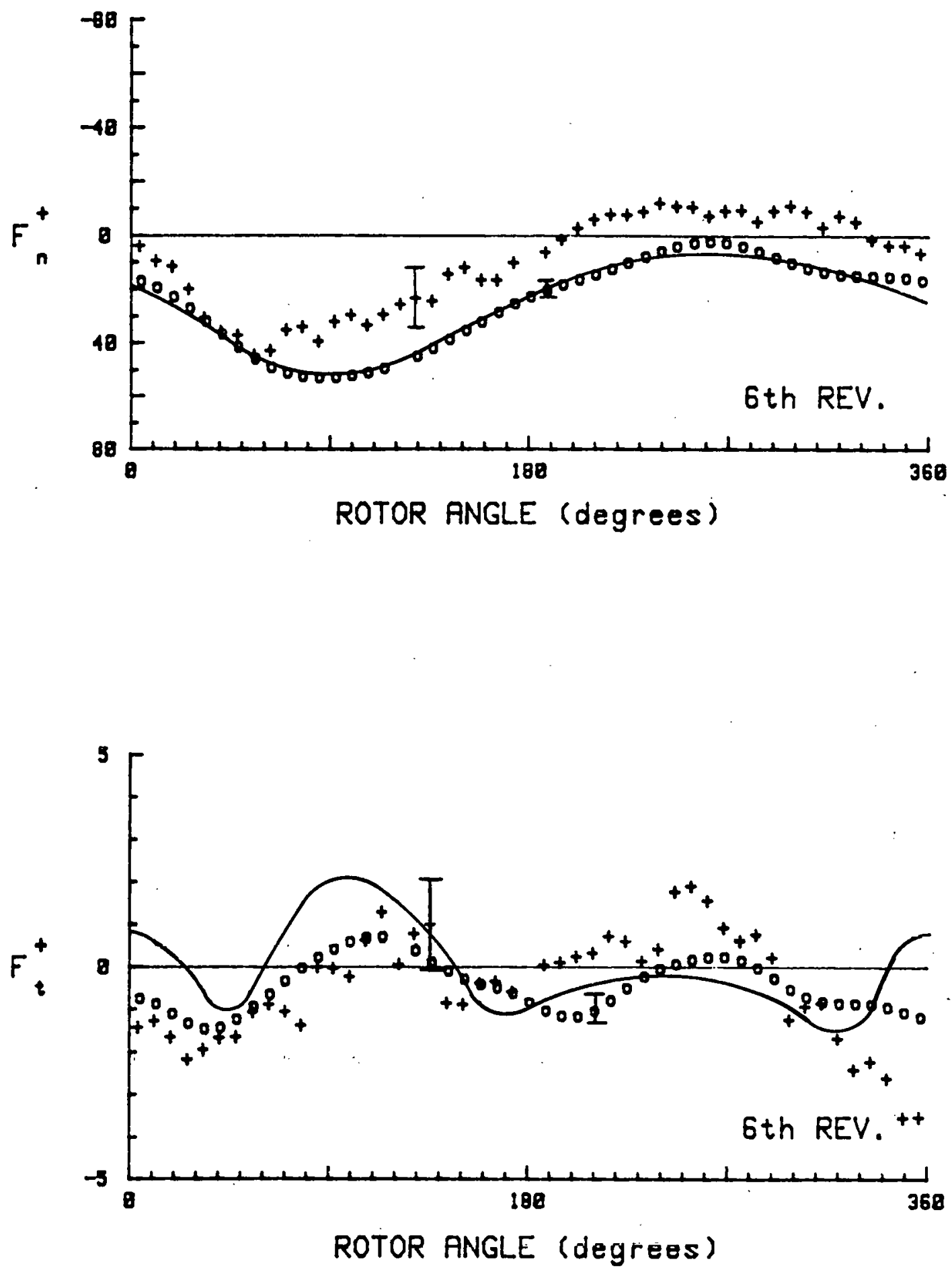

Fig. 4.5.3 Non-Dimensional Blade Forces

$(\mathrm{TSR}=7.6, \mathrm{C} / \mathrm{R}=0.25, \mathrm{NB}=1$, Pressure Data + , Strain Gage 0, VDART2 -) 
Fair agreement exists for the normal force $\left(\mathrm{F}_{n}^{+}\right)$results between the two experimental sets of data and the VDART2 analysis. The agreement between the strain gage data and the analysis is typical of that noted previously by Strickland $(1981(\mathrm{a}))$ for two bladed rotors with $\mathrm{C} / \mathrm{R}=0.15$.

The tangential forces $\left(\mathrm{F}_{t}^{+}\right)$are an order of magnitude smaller than the normal forces and thus tend to be more difficult to measure. The agreement between the integrated pressure measurements and the strain gage measurements is seen to be reasonably good in the upstream region between $0^{\circ}$ and $180^{\circ}$. The integrated pressure measurements typically tend to produce much larger values of $\mathrm{F}_{t}^{+}$than do the strain gage measurements in the region of $180^{\circ}$ to $360^{\circ}$. The reason for this is presently not well understood. The VDART2 analytical predictions when compared with the strain gage data are again typical of those noted by Strickland $(1981(\mathrm{a}))$. The peak value of $\mathrm{F}_{t}^{+}$ predicted in the upstream region for a tip-to-windspeed ratio of 2.5 tends to be high in comparison to the strain gage measurements. This discrepancy can be traced to an inadequate dynamic stall model in the VDART2 analysis. Several things can be sald with regard to the accuracy of the various force data. The strain gage mèsurements appear to yleld llie best results In that the data are smooth and vary in a continuous fashion from one cycle to the next. This is readily seen, for instance, by comparing Figures 4.3.4 and 4.3.5 with 4.4 .2 abd 4.4.3. The strain gage data are also quantitatively similar to strain gage data taken previously by Strickland (1982(a)) for slightly different cases. The strain gage data are subject to error due to aspect ratio corrections which were only approximately made in the present work. In addition, some error may have been introduced due to minor tangential accelerations of the blade caused by play in the drive train. These sources of error are not considered in the indicated error bars. The integrated pressure data, on the other hand, is subject to errors associated with local static pressure variations due to small 
vertical displacements of the blade as it moves through the water. This change in reference pressure affects the normal force since the blade was run once in each direction through the tow tank to obtain pressures on both sides of the airfoll. The numerical integration procedure used as well as the number of pressure taps can also significantly affect the integrated pressure results. In the present work the number of pressure taps may not have been adequate. Another source of error which can be significant at high tip to windspeed ratios is blade toe-in or toe-out. A toe-in or out of 5 degrees will, for instance, change the magnitude of $F_{t}$ by about 2.5 at a tip to windspeed ratio of 7.6 .

In summary, the integrated pressure measurements do not yield sufficiently accurate tangential force data and should be improved by eliminating some of the sources of experimental error. The normal forces obtained from integrated pressure measurements are, on the other hand, of fair quality.

\subsubsection{Pressure Data}

In this section the pressure data are analyzed to extract information concerning the phenomenon of dynamic stall. The data indicate that dynamic stall occurs primarily at the lowest tip-to-windspeed ratio of 2.5 although some stall may occur at the tip-to-windspeed ratio of 5.1 The data shown in Figures 4.5.4-4.5.10 obtained at a tip-to-windspeed ratio of 2.5 during the second revolution of the NACA 0015 blade. The data presented in this section are the result of a single run to avold any averaging of the pressure pulse moving across the airfoll. The nondimensional pressure coefficient is defined by equation 4.3 .1 . 

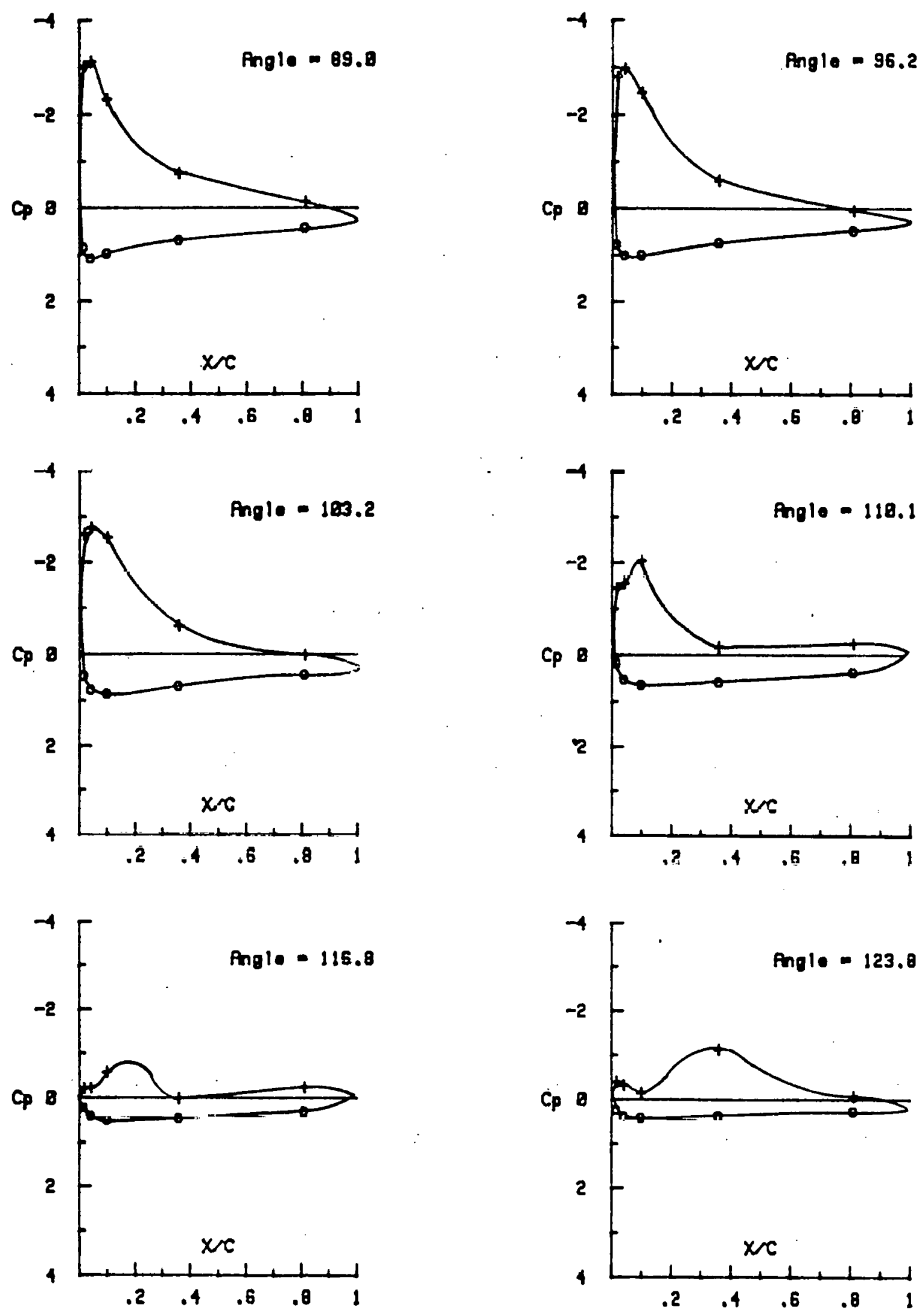

Fig. 4.5.4 Effect of Rotor Angle on Pressure Coefficient $(\mathrm{TSR}=2.5, \mathrm{C} / \mathrm{R}=0.25, \mathrm{~N}=1$, Outer Surface 0 , Inner Surface + ) 

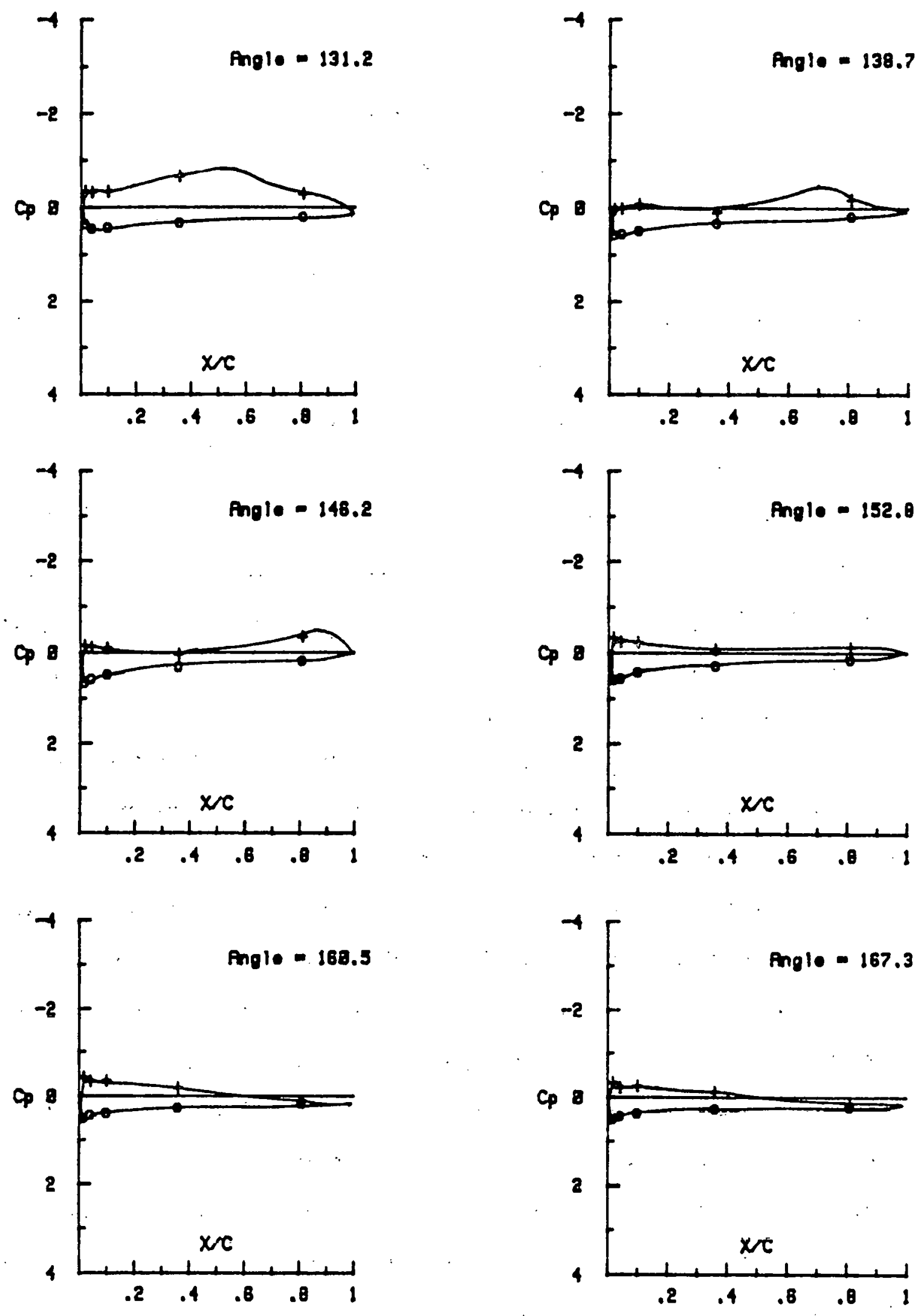

Fig. 4.5.5 Effect of Rotor Angle on Pressure Coefficient $(\mathrm{TSR}=2.5, \mathrm{C} / \mathrm{R}=0.25, \mathrm{~N}=1$, Outer Surface 0 , Inner Surface +) 
As seen in Figure 4.5 .4 , at a rotor angle of $110.1^{\circ}$ there is a flattening of the $C_{p}$ curve over the aft inner. surface of the airfoil indicating separation. Simultaneously the suction peak begins to decrease in magnitude. At a rotor angle of $116.8^{\circ}$ the angle of attack is approximately $23^{\circ}$ and the airfoll appears to be fully stalled over the inner surface. In the vicinity of this rotor angle it is believed that a vortex is shed at the nose and begins moving along the surface of the airfoll. At a rotor angle of $123.8^{\circ}$ this vortex appears to be paseing through the vicinity of $\mathrm{x} / \mathrm{C}=$ 0.36 and has significantly affected the pressure on the surface of the airfoll at this location. The alrfoll remains in a stalled condition until a rotor angle of approximately $150^{\circ}$. At this point the flow reattached as Indicated by the slight suction over the nose of the alrfoll as shown in Figure 4.5 .5 at a rotor angle of $152.8^{\circ}$.

Figures 4.5 .6 and 4.5 .7 show the $C_{p}$ curves at rotor angular positions where the outer surface of the alrfoll is experiencing stull.

Separation of the flow over the aft portion of the airfoil appears to begin at a rotor angle of $264.9^{\circ}$. At a rotor angle of $271.9^{\circ}$ the angle of attack is approximately $-23^{\circ}$ and the blade is fully stalled over the outer surface. As in the case of the Inner surface stall described above, a vortex is shed at the nose of the airfoil and begins moving along the surface. The effect of this vortex on the pressure tap located at $x / C-0.36$ may be seen at a rotor angle of $279.4^{\circ}$. The flow appears to have near 1y reattached at a rotor angle of $300.4^{\circ}$. However, the $C_{p}$ value at the $x / C$ location of 0.81 indicates a strong pressure disturbance probably due to the vortex shed at the onset of stall.

Figures 4.5.8 - 4.5.10 show the effect of angle of attack on the pressure coefficlent. The bars indicate suspected regions of stall. In. 

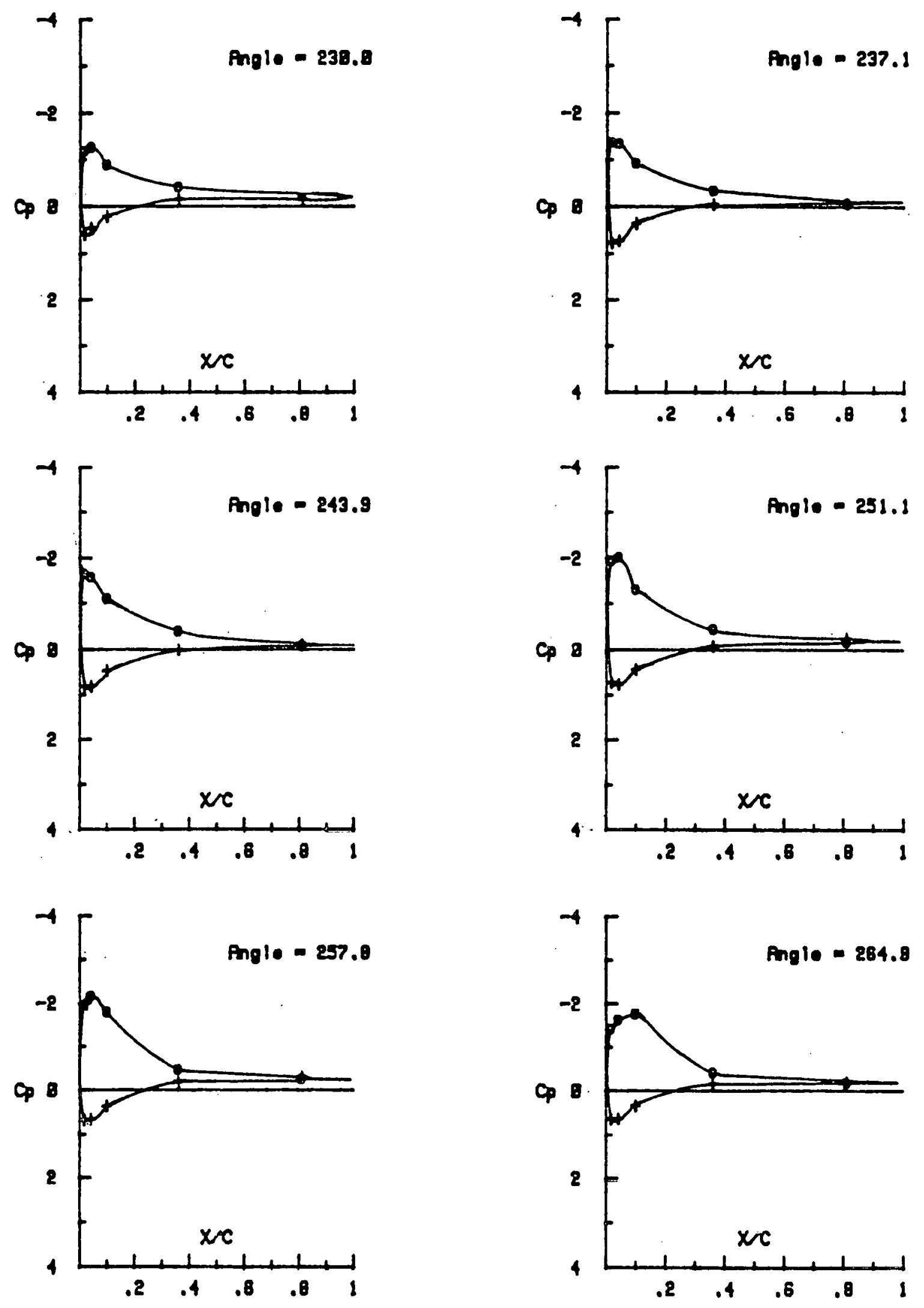

Fig. 4.5.6 Effect of Rotor Angle on Pressure Coefficient (TSR $=2.5, C / R=0.25, N=1$, Outer Surface 0 , Inner Surface + ) 

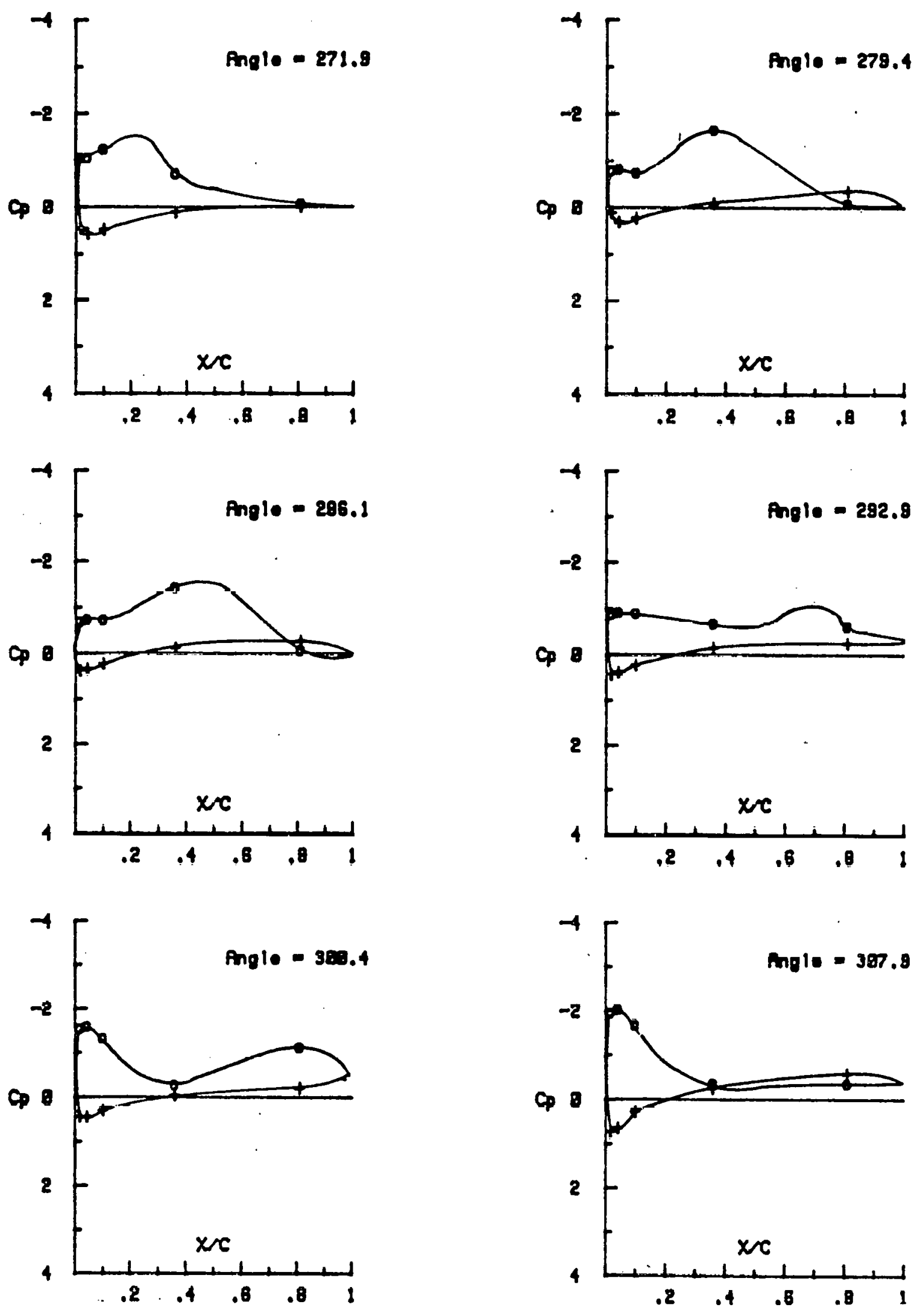

Fig. 4.5.7 Effict of Rotor Angle on Pressure Coefficient $(\mathrm{TSR}=2.5, \mathrm{C} / \mathrm{R}=0.25, \mathrm{~N}=1$, Outer Surface 0 , Inner Surface + ) 

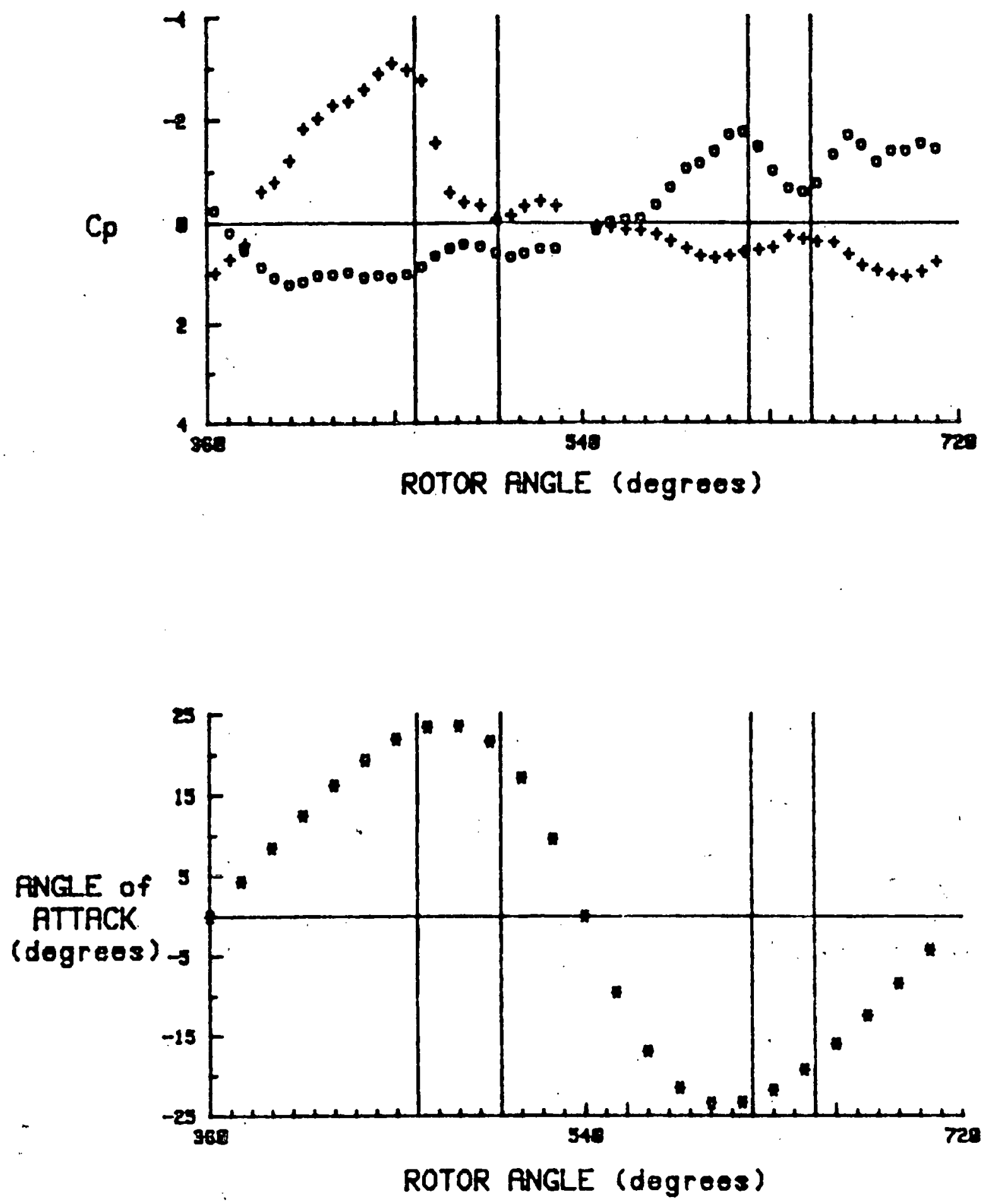

Fig. 4.5.8 Effect of Angle of Attack on the Pressure Coefficient for the Nose Region $(0.017<\mathrm{X} / \mathrm{C}<0.1)$ $(\mathrm{TSR}=2.5, \mathrm{C} / \mathrm{R}=0.25, \mathrm{NB}=1$, Outer surface 0 , Tnner Surface +) 

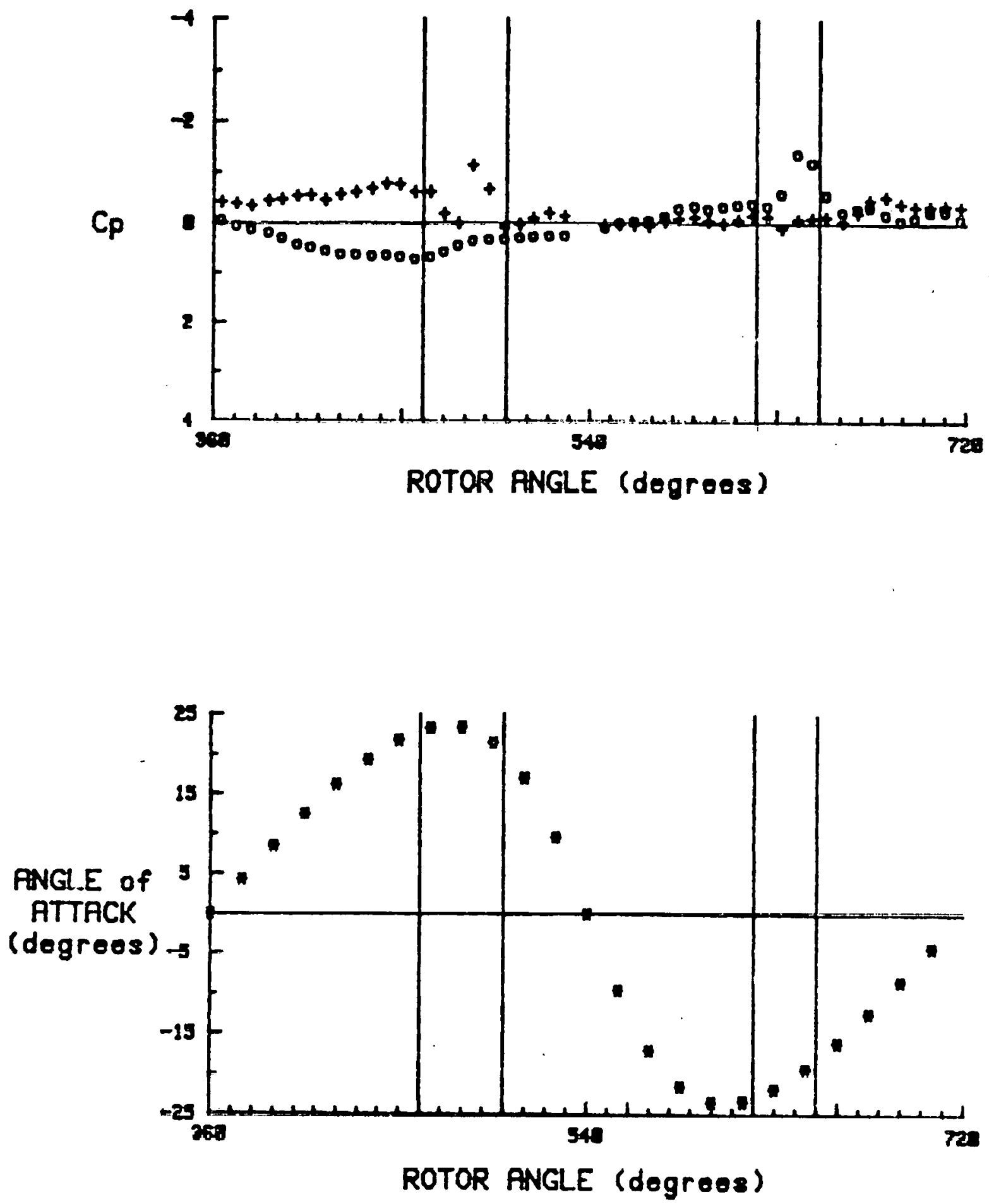

Fig. 4.5.9 Effect of Angle of Attack on the Pressure Coefficient for $\mathrm{X} / \mathrm{C}=0.36$

(TSR $=2.5, C / R=0.25, \mathrm{NB}=1$, Outer Surface 0 , Inner Surface + ) 

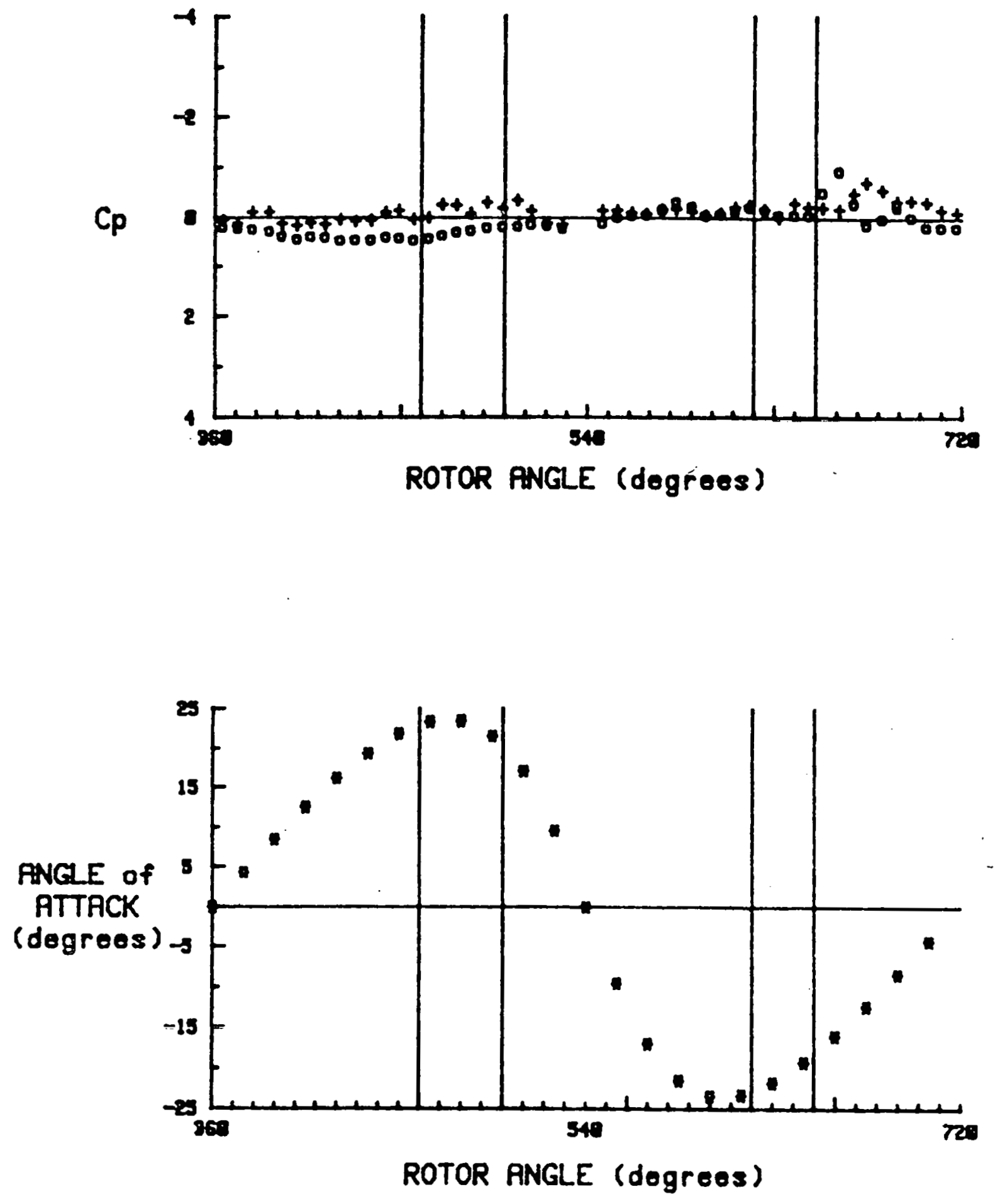

Fig. 4.5.10 Effect of Angle of Attack on the Pressure Coefficient for $\mathrm{X} / \mathrm{C}=0.81$ $(\operatorname{TSR}=2.5, C / R=0.25, N B=1$, Outer Surface 0 , Inner Surface +) 
Figure 4.5.8 the maximum value of the pressure coefficient over the first three pressure taps is plotted versus the rotor position. The $C_{p}$ values on the inner surface increase with increasing angle of attack until dynamic stall occurs. At this point, a dramatic loss of suction is observed until the flow reattaches. This effect is also observed in the region of outer surface stall. Figure 4.5 .9 shows the effect of dynamic stall on the pressure coeffictent at $x / C=0.36$. The abrupt 1ncrease in the magnitudc nf the pressure coeffictent in the regions of stall indicates passage of a vortex through this vicinity. This phenomenon is observed in both regions nf stall.

A comparison of Figures 4.5 .9 and 4.5.10 indicates a time delay between the effect of the moving, vortex on the pressure coefficient at $x / C$ locations of 0.36 and 0.81 . The motion of this vortex can also be seen in figures 4.5.4 through 4.5.7. From these figures it can be seen that the vortex moves with a nearly constant velocity tuwaid the trailing cdgc of the airfoil at about $30 \%$ of the airfoll speed (rotor-tip-speed) or in other words the airfo1l moves about 3.5 chord lengths wh1le the vortex moves from the nose to the trailing edge. 


\section{CONCLUSIONS and RECOMMENDATTUNS}

In this chapter, a summary of the work performed is presented followed by a list of conclusions. Also, recommendations for future work are discussed.

\subsection{Summary of the Analytical Study}

The long-term goal for the present investigation is the development of a numerical model of dynamic stall as it occurs on Darrieus turbines. The current project has produced a numerical model theoretically capable of accomplishing this. The model is capable of predicting, with reasonable accuracy, steady and unsteady flows over alfolls with attached boundary layers and quasi-steady, separated flows.

The principal difficulty yet to be overcome before calculations of unsteady, separated flows can be made is the coupling of the viscid and inviscid calculations on a step-by-step basis. DYNA2 must incorporate this feature before any attempt can be made to simulate a dynamically stalled airfoil.

of lesser importance is the issue of the bastc surface modeling element. If higher order curved panels are utilized, then significantly higher computation costs will be incurred. W1thout their adoption, the DYNA2 w111 lack the rellability and generality needed of an aerodynamic design tool. It is suggested that the current planar elements be preserved during the . continued development of DYNA2. The incorporation of advanced pane1 elements can be made during the final refinements. 


\subsection{Summary of the Experimental Investigation}

The primary purpose of the investigation was to obtain instantaneous pressure distributions which are characteristic of the Darrieus turbine. In addition, measurements of transient aerodynamic blade forces were made using strain gage instrumentation.

From the measurements, it is apparent that dynamic stall is prevalent at a tip-to-windspeed ratio of 2.5 . No stall was observed at tip-towindspeed racios of 5.1 aud 7.6 .

At the lip-to-windspeed ratio of 2.5 , dynamic stall norurs over the Inner surfaces at rotor angles of approximately $100^{\circ}<\theta<140^{\circ}$ corresponding to angles of attack of $20^{\circ}<\alpha<23^{\circ}$. Dynamic stall over the outer surface occurs at rotor angles of approximately $265^{\circ}<\theta<290^{\circ}$ corresponding to angles of attack of $18^{\circ}<\alpha<23^{\circ}$.

Boundary layer separation appears to proceed from the trailing edge to the leading edge. This is accompanied by the shedding of a vortex at the nose and the subsequent movement of the vortex over the stalled surface. The vortex moves toward the trafling edge at about one-half the alrfoll 8 peed.

For future experimental studies, it is recommended that the number of pressure taps be doubled and that additional probes be located on the opposite side of the airfoll. 


\section{REFERENCES}

Arena, A. V., Mueller, T., 1980; "Laminar Separation, Transition, and Turbulent Reattachment Near the Leading Edge of Airfoils," AIAA Journa1, 18(7), pp. 747-753.

Ashley, H.; "Machine Computation of Aerodynamic Loads in Linear and Nonlinear Situations," Fluid Dynamics Research Lab. Rept. 66-5, AFOSR 66-1440.

Cebecc1, T. and Smith, A. M. 0., 1974; Analysis of Turbulent Boundary Layers, Academic Press.

Cebecc1, T. and Bradshaw, P., 1977; Momentum Transfer in Boundary Layers, McGraw-Hill.

Clements, R. R. and Mau11, D. J., 1971; "The Representation of Sheets of Vorticity by Discrete Vortices," Prog. In Aerospace Sclences, 16, p. 129 .

Coles, D., 1956; "The Law of the Wake in the Turbulent Boundary Layer," JFM, 1, pp. 191-226.

Coles, D. and Hirst, E. A., 1969; Computation of Turbulent Boundary Layers, 1968 AFOSR-RFP, Vo1. 2, Stanford University.

Daneshyar, H. and Mugglestone, P. R., 1978; "An Integra1 Method for Calculating the Development of a Turbulent Boundary Layer with a Fluctuating Mainstream," Iranian J. of Sclence and Technology, 7, pp. 17-35.

Djojodihardjo, R. H. and Widaal, S. E., 1969; "A Numerical Method for the Calculation of Nonlinear, Unsteady Lifting Potential Flow Problems," AIAA J., 7, pp. 2001-2009.

Dwyer, H. A. and McCroskey, W. J., 1971; "Crossflow and Unsteady Boundary Layer Effects on Rotating Blades," AIAA J., 9, pp. 1498-1505.

Fanucc1, J. B. and Walters, R. E., 1976; "Innovat1ve W1nd Mach1nes: The Theoretical Performance of a Vertical Axis Wind Turbine, "Proceedings - Vertical Axis Wind Turbine Technology Workshop, Sandia Laboratory Report SAND76-5586.

Gormont, R. E., 1973; "A Mathematica1 Mode1 of Unsteady Aerodynamics and Radial Flow for Application to Hellcopter Rotors," USAAMRDL Technical Report 72-67.

Graham, G. M., 1982; "Measurement of Instantaneous Pressure Distributions and Blade Forces on an Alrfoil Undergoing Cycloldal Mntion," M.S. Thesis, Texas Tech Universicy. 
Green, I. E., Weeks, D. J., and Brooman, J. W. F., 1973; ARC R\&M, 3791 .

Ham, N. D., 1968; "Aerodynamic Loading on a Two-Dimensional Airfoil During Dynamic Sta11," AIAA J., 6, p. 1927-1934.

Head, M. R., and Patel, V. C., 1969; "Improved Entrainment Method for Calculating Turbulent Boundary Layer Development," ARC R\&M 3643.

Houdeville, R. and Couste1x, J., 1979, "Premiers Resultats D'une Etude sur les Couches Limites Turbulentes en Encoulement Pulse Avec Gradient de Pression Moyen Defavorable," 15E Collogue D;Aerodynamique, AAAF NT-79-05, 1978. Translated into English in NASA TN-75799, 1979.

Kadlec, E. G., 1980; "Overview of Current and Future Design Characteristics (of VAWT's), Proceedings - VAWT Design Technology Seminar for Industry, Sandia Laboratory Rept. SAND80-0984.

Karamchet1, K., 1966; Principles of Idea1-Fluld Aerodynamics, John Wdley and Sons.

Karlson, S. K. F., 1959; "An Unsteady Turbulent Boundary Layer," J. Fluid Mechanics, 5(4).

Kim, J., Kline, S. J., and Johnston, J. P., 1980; "Investigation of Separation and Reattachment of a Turbulent Shear Layer: Flow Over a Backward-Facing Step," Rpt. MD-37, Thermosciences Div., Mech. Engrg. Dept., Stanford University, 1978. See also TASME, J. Fluids Engrg., 102, pp. 392-308, 1980.

K1.imas, P. C., 1980; "Vertica1 Axis Wind Turbine Aerodynamic Performance Prediction Methods," Proceedings = VAWT Design Technology Seminar for Industry, Sandia Laboratory Rept. SAND80-0984.

Klimas, P. C., 1980; "Possible Aerodynamic Improvements for Future VAWT Systems," Proceedings = VAWT Design Technology Seminar for Industry, Sandia Laboratory Reporrt SAND80-0984.

Kraus, W., 1978; "Panel Methods in Aerodynamics," Numerical Methods in Fluid Dynamics, McGraw-Hi11, Editors Wirz and Smolderen, pp. $237-297$.

Lyrio, A. A.jFcroigor, J. H., and k.line, S. I., 1981; "An Tntegral Method for the Computation of Steady and Unsteady Turbulent Boundary Layer Flows, Including the Transitory Stall Regime in Diffusers," Stanford University Report PD-23.

Martin, J. M., Empey, R. W., McCroskey, W. J., and Caradonna, F. S., 1974; "An Experimental Analysis of Dynamic Stall on an Oscillating Airfoil," J. Am. Helicopter Soc., 19, pp. 26-32.

McCroskey, W. J. and Phillippe, J. J., 1975; "Unsteady Viscous Flow on Oscillating Airfoils," AIAA J., 13, pp. 71-79. 
Moore, F. K., 1958; "On the Separation of the Unsteady Boundary Layer," Boundary Layer Research, Gortler, H., (Ed.), Springer-Verlag, Berr11n, Pp. 296-310.

Myring, D. F., 1970; RAE Tech Report 70147.

Nash, J. F. and Pate1, V. C., 1969; "Improved Entrainment Method for Calculating Turbulent Boundary Layer Development," NASA CR-2546.

Nash, J. F., Carr L. W., and Singleton, R. E., 1975; Unsteady Turbulent Boundary Layers in Two-Dimensional Incompressible Flow," AIAA J., 13, pp. 176-183.

Nash, J. F., Carr, L. W., and Singleton, R. E., 1975; Unsteady Turbulent Boundary Layers in Two-Dimensional Incompressible Flow," AIAA J., 13, pp. 167-173.

Nash, J. F. and Scruggs, R. M., 1978; "Unsteady Boundary Layers with Reversal and Separation," Proceedings = Unsteady Aerodynamics, AGARD $\mathrm{CP}-227$, pp. 18.1-18.12.

Newman, B. G., 1951; "Some Contributions to the Study of the Turbulent Boundary Layer Near Separation," Austr. Dept. Supply Rept. ACA-53.

Oler, J. W., 1976; "An Investigation of a Numerical Method for the Exact Calculation of Unsteady Airloads Associated with Wing Interaction Problems," M.S. Thesis, University. of Texas - Austin.

Roberts, W. B., 1980; "Calculation of Laminar Separation Bubbles and Their Effect on Airfoll Performance," AIAA Journal, 18(1), pp. 25-31.

Rott, N., 1956; "Unsteady Flow in the Vicinity of a Stagnation Point," Quart. J. of App1. Math., 13, pp. 444-451.

Sandborn, V. A. and K1ine, S. J., 1961; "Flow Models in Boundary Layer Stall Inception," Journal of Basic Engineering, Trans. ASME, pp. 317-327.

Sarpkaya, T. and Schoaff, R. L., 1979; "Inviscid Model of Two-Dimensional Vortex Shedding by a Circular Cylinder, " AIAA J., $17(11)$, pp. 1193-1200.

Sears, W. R., 1956; "Some Recent Developments in Airfoll Theory," 23, pp. 490-499.

Simpson, R. L., Strickland, J. H., and Barr, P. W., 1977; "Features of a Separating Turbulent Boundary Layer in the Vicinity of Separation," JFM, 79, pp. 553-594.

Singleton, R. E. and Nash, J. F., 1974; "Method for Calculating Unsteady Turbulent Boundary Layers in Two- and Three-Dimensional Flows," AIAA Journal, 12(5), pp. 590-595. 
Smith, A. M. O. and Hess, J. L., 1962; "Calculation of the Nonlifting Potential Flow About Arbitrary Three-Dimensional Bodies," Douglas Rept. No. E.S. 40622 .

Strickland, J. H., 1976; "Aerodynamics of the Darrieus Turbine," Proceedings $=$ Vertical Axis Turbine Technology Workshop, Sandia. Laboratory Rept. SAND76-5586.

Strickland, J. H., Webster, B. T., and Nguyen, T., 1979; "A Vortex Model of the Darrieus Turbine: An Analytical and Experimental Study," J. Fluids Engineering, 101(12), pp. 500-505.

Strickland, J. H., 1981; "Vortex Model of the Darrieus Turbine: An Analytical and Experimental Study," Sandia Laboratory Report

SAND81-7017, 165 pages.

Strickland, J. H., Smith T., and Sun, K., 19̆8̌l; "A Usèr's Mảnual for the Vertical Axis Wind Turbine Code VDART2," Sandia Laboratory Report SAND81-7U39, 50 pages.

Summa, J. M., 1976; "Potential Flow About Impulsively Started Rotors," J. Aircraft, 13, pp. 317-319.

Telionis, D. P., 1975; "Calculations of Time-Dependent Boundary

Layers," Unsteady Aerodynamics, R. B. Kinney (Ed.), Vol. 1, p. 155.

Wilson, R. E., 1978; "Vortex Sheet Analysis of the Giromill," J. Fluids Englneering, 100(3), pp. 340-342.

Woudward, F. A., 1973; "An Improved Method for the Aerodynamic Analysis of Wing-Body-Tall Configurations in Subsonic and Supersonic Flow," NASA Cr-2228. 
7. APPENDICES 


\section{APPENDIX A \\ FUNDAMENTALS OF POTENTIAL AERODYNAMICS}

As an aid to understanding the unsteady aerodynamic solution method described in the preceeding chapters, a brief introduction to the fundamentals of low speed aerodynamic theory will now be given. Particular attention will be directed to the somewhat subtle aspects of lifting potential flow theory. Most of the theoretical relationships will be stated without proof. Their physical significance will be emphasized, however, and references given where the derivations and additional discussion may be found.

\section{A.1 The Governing Equations}

We are concerned with finding an Eulerian or "field" description of the motion of an ideal fluid through which one or more bodies move. An Ideal fluid is defined as one that is inviscid, homogeneous, and incompressible. These assumptions are compatible with the physical realities of low speed aerodynamics.

The objective is to determine a set of governing equations which specify the relationship between the unknown vector velocity and scalar pressure fields. With these relationships and properly specifled initial and boundary conditions, the unknown flelds may be decermined. The basis for these equations are the natural laws of conservation of mass and momentum.

Newton's second law of motion states that at any instant, the rate of change of momentum of any mechanical system is equal to the force acting on it at that instant. This requirement is described by 
Euler's equation (see Karamcheti, pp. 175-190);

$$
\frac{\mathrm{D}}{\mathrm{Dt}}(\rho \overrightarrow{\mathrm{U}})=\rho \overrightarrow{\mathrm{F}}-\nabla \mathrm{P}
$$

or

$$
\rho\left\{\frac{\partial \vec{U}}{\partial t}+[\vec{U} \cdot \nabla] \vec{U}\right\}=\rho \vec{F}-\nabla P
$$

mass $\mathrm{x}$ acceleration $=\begin{aligned} & \text { body } \\ & \text { force }\end{aligned}+\begin{aligned} & \text { pressure } \\ & \text { force }\end{aligned}$.

Conservation of mass requires that the mass of an infinitesimal fluid element be constant. Since the fluid is incompressible, the mass per unit volume is a constant and it is sufficient to require that the velocity field not diverge. This is expressed by

$$
\nabla \cdot \vec{U}=0
$$

The vector conservation of momentum requirement provides three nonlinear equations, while the continuity equation yields a linear fourth. The simultaneous solution of these is sufficient to determine the unknown velocity components and pressure. The actual determination of that solution is quite difficult in most cases, however, due to the nonlinearity of the momentum equations.

\section{A. 2 Irrotationa1 Motion}

The Helmholtz theory of vortex motion states that in the motion of an ideal fluid through an irrotational force field, the material rate of change of the circulation around any fluid surface element is zero. This is expressed as (Karamcheti, p. 239)

$$
\frac{\mathrm{D}}{\mathrm{D} t} \oint_{\mathrm{C}_{\mathrm{S}}} \overrightarrow{\mathrm{U}} \cdot \overrightarrow{\mathrm{d} s}=\frac{\mathrm{D}}{\mathrm{D} t} \Gamma_{\mathrm{C}_{\mathrm{S}}}=0 .
$$


Kelvin's circulation theorem provides a slightly stronger statement of the same concept; in the motion of an ideal fluid through an irrotational force field, the circulation around a closed fluid curve remains constant for all time. This theorem is expressed as (Karamchet1, p. 242)

$$
\frac{\mathrm{D}}{\mathrm{Dt}} \oint_{\mathrm{C}} \overrightarrow{\mathrm{U}} \cdot \overrightarrow{\mathrm{d} s}=\frac{\mathrm{D}}{\mathrm{Dt}} \Gamma_{\mathrm{C}}=0
$$

Application of Stokes theorem on rotation to the Kelvin-Helmholtz circulation theorem ylelds a significant result. Through purely kinematical arguments, Stokes demonstrated that for any vector field in a region, $R$, the vorticity flux through an open surface contained in $R$ is equal to the circulation around the closed curve which bounds the open surface. This is stated as (Karamchet1, p. 132)

$$
\iint_{S} \vec{n} \cdot \overrightarrow{r_{0}} d S=\int_{S} \vec{U} \cdot d \vec{s}
$$

where

$$
\vec{\zeta}=\nabla \times \vec{U}
$$

Combining this with the Kelvin-Helmholtz theorem yields

$$
\frac{D \Gamma}{D t}=\frac{D}{D t} \iint_{S} \vec{n} \cdot \vec{\zeta} d S=0
$$

Therefore, one concludes that for an Ideal fluid in an Irrotational force fleld, the vorticity flux through any fluid surface element is a constant. Furthermore, if that vorticity flux is zero for any instant, it must be zero for all times. This is the reason for 
generally assuming that ideal fluid motion is started from a state of rest or uniform motion in which case, $\vec{\zeta}=0$ over the entire region occupied by the fluid. Since the vorticity is equal to twice the angular rotation rate of the fluid, it is said that the motion must be rotation free or irrotational for all times after the starting instant.

The requirement of irrotationality for all times is a necessary and sufficient condition to guarantee the existance of a velocity potential, i.e.,

$$
\vec{\zeta}=\nabla \times \vec{U}=0
$$

therefore,

$$
\overrightarrow{\mathrm{U}}=\nabla \Phi
$$

The existence of a velocity potential has several important consequences with respect to the governing equations.

Euler's equation was given previously as

$$
\rho\left\{\frac{\partial \vec{U}}{\partial t}+[\vec{U} \cdot \nabla] \vec{U}\right\}=\rho \vec{F}-\nabla P
$$

This may be rewritten as

$$
\frac{\partial \vec{U}}{\partial t}+\nabla\left(\frac{U}{2}\right)^{2}-\vec{U} \times[\nabla \times \vec{U}]=\vec{F}-\nabla\left(\frac{p}{\rho}\right) .
$$

Assuming an irrotational motion and force fleld yields

$$
\frac{\partial}{\partial t} \nabla \Phi+\nabla\left[\frac{\nabla \Phi \cdot \nabla \Phi}{2}\right]=\nabla \Omega-\nabla\left(\frac{\rho}{\rho}\right)
$$

or 


$$
\nabla\left\{\frac{\partial \Phi}{\partial t}+\frac{(\nabla \Phi)^{2}}{2}-\Omega+\frac{P}{\rho}\right\}=0
$$

where

$$
\nabla \Phi=\vec{U} \text { and } \nabla \Omega=\vec{F}
$$

After integration of the last expression, one arrives at

$$
\frac{\partial \Phi}{\partial t}+\frac{1}{2}(\nabla \Phi)^{2}-\Sigma+\frac{P}{\rho}=E(t)
$$

which is the unsteady Bernoulli's equation.

The conservation of mass requirement was previously given as

$$
\nabla \cdot \vec{U}=0
$$

Substitution of the velocity potential yields

$$
v^{2} \dot{\Phi}=u
$$

which is the familiar Laplace equation.

Prior to the accumption of irrotationality and, thereby, the existence of a velocity potential, there were four equarions for the unknown vector velocity and scalar pressure tlelds. Now, there are just two equations for the scalar velocity potential and pressure flelds. Furthermore, the governing equation is now the linear Laplace equation. Since it is lincor, complicated colutione for toy be constructed from the superposition of elementary ones. Once $\Phi$ is determined, the pressure field may be found from Bernoulli's equation.

\section{A.3 The Circulation Theory of Lift}

Consider the irrotational flow of an ideal fluid past a two-dimen- 
sional body. Since the body is two-dimensional, the flow field is said to be doubly connected and the possibility exists for a finite circulation around a circuit which includes the body.

d'Alembert considered the case in which the circulation is specified to be zero and came to the surprising conclusion that the net resistance forces of the fluid on the body are zero. This is commonly known as d'Alembert's paradox.

Kutta and Joukowski independently considered the case of nonzero circulation and found that a finite lift force was produced. The result is known as the Kutta-Joukowski theorem of $11 \mathrm{ft}$ and is stated as: If there is a circulation of magnitude $\Gamma$ around the cylinder and if the undisturbed velocity at infinity has the magnitude $U_{\infty}$, then a lift exists with a magnitude of $\rho U_{\infty} \Gamma$ per unit span.

The requirement of a net circulation for the generation of lift complicates the potential flow problem. From topology considerations (Karamcheti, pp. 252-263), it may be shown that a finite circulation can only exist for an irreducible circuit in a multiply connected region. A consequence of the multiple connectivity is that the potential field is not unique unless the circulations around the irreducible circuits are specified. Additional relationships based upon physical experience must be found to determine the circulation strengths, since the previously applied natural laws are of no help. In addition, consideration must be given to the Kelvin-Helmholtz theorem and how the circulation was established in the first place. A.4 Two-Dimensional Airfoil Theory 
If the flow field around an airfoil having a sharp trailing edge is calculated assuming zero circulation, a streamline pattern similar to the one shown in Figure A.1 results. As a consequence of the surface gradient discontinuity at the trailing edge, a velocity singularity exists at that point. Physical experience has demonstrated that an infinite velocity cannot exist in any real fluid with a finite viscosity, Realization of this deficiency in the 1 deal fluid representation led Kutta and Joukowski to propose that the circulation about the airfoil be such that the flow from the tralling edge be smooth and finite. This is typically known as the Kutta condition. It provides the additional relationship which is necessary to uniquely specify the circulation strength and potential field. Now consider a two-dimensional airfoll at rest in an ideal fluid. Since the fluid velocity is everywhere zero, the circulation around the airfoil is zero. At the initial instant, $t=0$, the fluid is Instantaneously brought into motion such that at infinity, $U=U_{\infty}$. The Kutta condition requires that the fluid flow smoothly off of the trailing edge and, consequently, that there be a finite circulation around the alrfoll. The Kelvin-Helmholtz theorem, however, requires that the circulation about any closed curve in the fluid be zero. The simultaneous satisfaction of these apparently contradictory conditions is accomplished by the generation of a trailing wakc which formo the starting vortex illustrated in Figure A.2.

For any incremental time interval, $\delta t$, the vorticity shed from the trailing edge into the wake is equal to the negative of the change in "bound vorticity" or equivalently, circulation strength about the air- 


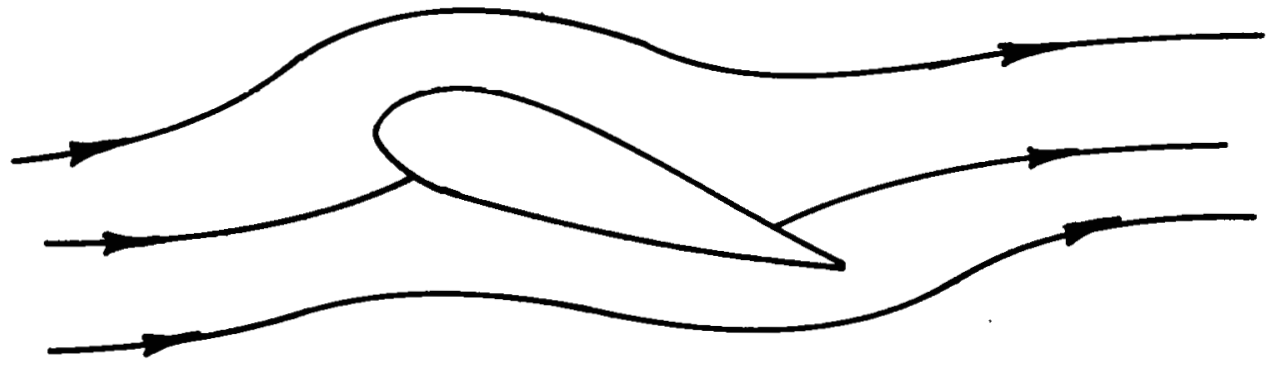

Figure A.1 Flow Field About an Airfoil

With Zero Circulation. 


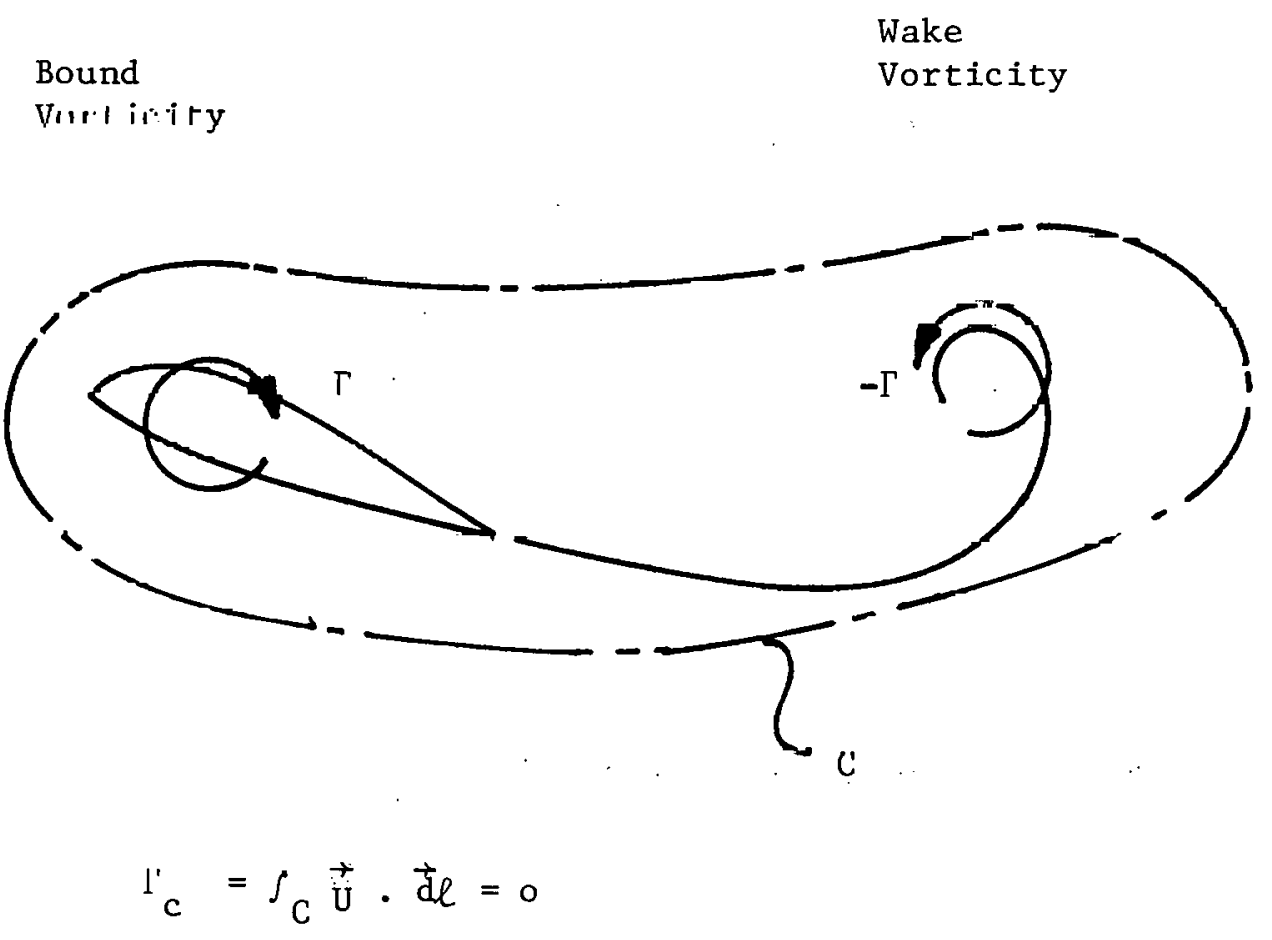

Figurc A.2 Flow About an AirFoil which Satisfies the Kutta Condition 
foil during that time interval. Referring to Figure A.2, this can be expressed mathematically as

$$
\delta \Gamma_{w}=-\frac{d \Gamma_{b}}{d t} \delta t
$$

Therefore, the net circulation around the wake is always the exact negative of the circulation around the airfoil, i.e.,

$$
\Gamma_{w}=\int_{0}^{T}-\frac{d_{b}}{d t} d t=-\Gamma_{b} .
$$

The wake surface is a sheet of potential discontinuity and, consequently, a boundary in the flow field. All closed fluid circuits of the Kelvin-Helmholtz type must include the entire lifting system of airfoll plus the trailing wake as shown in Figure A.2. The net circulation around these circuits, $\Gamma_{\mathrm{K}-\mathrm{H}}$, is zero for the starting instant and all later times.

\section{A.5 Three-Dimensional Airfoil Theory}

A similar situation exists for finite wings as illustrated in Figure A.3. Before the starting instant, the fluid is at rest and the circulation strengths about all closed fluid curves in the region are zero. After the starting instant, a unique circulation strength of the bound vortex is determined by the Kutta condition at the trafling edge. Since it is not possible for a vortex tube to end in a fluid, away from the boundaries, two tip vortices must be shed. Downstream, the tip vortices are jolned to the starting vortex. For the simplified flow situation depicted in Figure A.3,

$$
\left|\Gamma_{T}\right|=\left|\Gamma_{b}\right|=\left|\Gamma_{W}\right|
$$




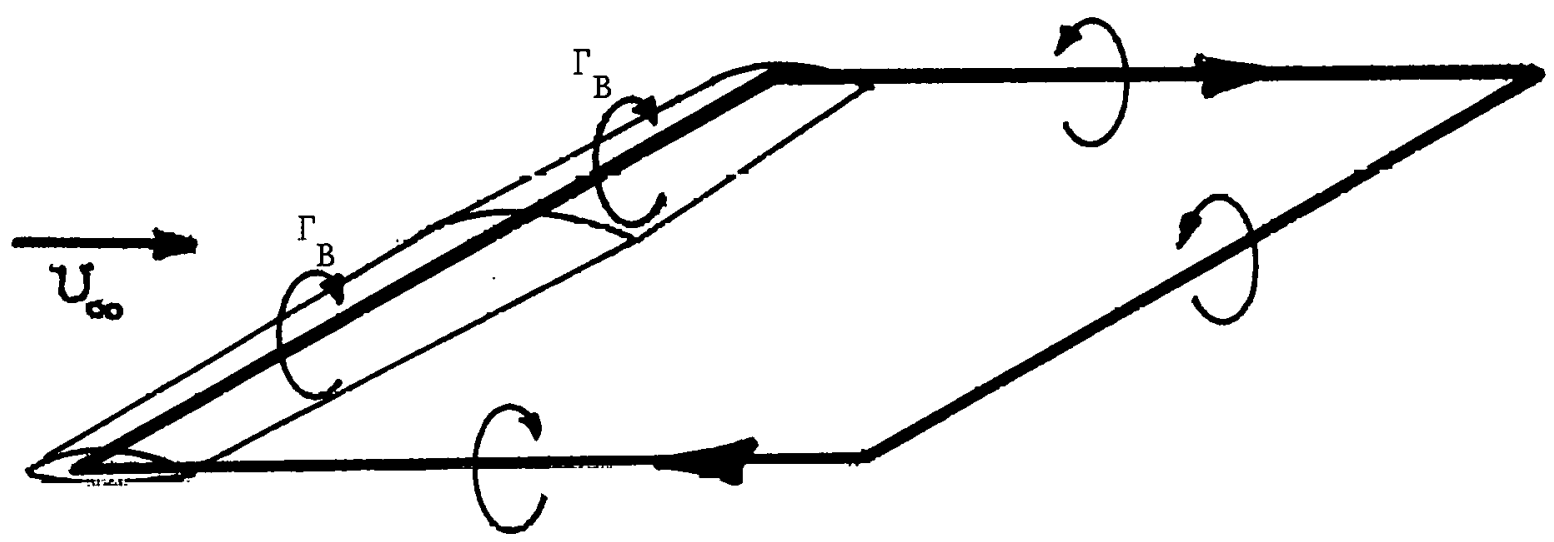

Figure A.3 Vortex System for a Finite Wing 
Consequently, any closed circuit about the complete lifting system will have zero circulation. This is sufficient to satisfy the Kelvin-Helmholtz theorem. 


\section{APPENDIX B}

AN INTEGRAL SOLUTION TO LAPLACE'S EQUATION

In this appendix, it will be shown that any solution to Laplace's equation may be expressed in terms of integrals of potential doublets and sources distributed over the boundaries of the solution domain. We will begin in the first section with a derivation of Green's theorem and in the following section demonstrate its application for the determination of the desired integral solution to Laplace's equation.

\section{B.1 Green's Theorem}

Gauss' divergence theorem expresses the equivalence of the divergence of a vector field within an enclosed volume with the flux of that vector field across the boundary surface. The mathematical statement of the theorem is

$$
\iiint_{V} \nabla \cdot \vec{A} d V=\oiint_{S} \vec{A} \cdot \vec{n} d S
$$

where $\vec{A}=$ any vector field

$$
\begin{aligned}
& V=\text { enclosed volume } \\
& \mathrm{S}=\text { bounding surface } \\
& \overrightarrow{\mathbf{n}}=\text { outwardly directed surface normal. }
\end{aligned}
$$

lior the vectur Field, $\vec{A}$, we may choose

$$
\overrightarrow{\mathrm{A}}=\phi \nabla \phi^{\prime}
$$

where $\phi$ and ' $\phi$ ' are continuous functions having finite first and second derivatives within the volume, V. Substitution for $\vec{A}$ in Equation B. 1 
yields

$$
\iiint_{V} \nabla \cdot \phi \nabla \phi^{\prime} \mathrm{dV}=\oiint_{S} \phi \nabla \phi^{\prime} \cdot \vec{n} \mathrm{dS}
$$

Equation B. 2 may be expanded to give

$$
\iiint_{V}\left[\nabla \phi \cdot \nabla \phi^{\prime}+\phi \nabla^{2} \phi^{\prime}\right] d V=\oiint_{S} \phi \frac{\partial \phi}{\partial n} d S
$$

which is known as Green's theorem in the first form.

We may alternately choose for $A$ in Equation B.l

$$
\overrightarrow{\mathrm{A}}=\phi^{\prime} \nabla \phi-\phi \nabla \phi^{\prime}
$$

Performing the substitution yields

$$
\iiint_{V} \nabla\left(\phi^{\prime} \nabla \phi-\phi \nabla \phi^{\prime}\right) \mathrm{dV}=\oiint_{S}\left(\phi^{\prime} \nabla \phi-\phi \nabla \phi^{\prime}\right) \cdot \overrightarrow{\mathrm{n}} \mathrm{dS}
$$

or

$$
\iiint_{V}\left(\phi^{\prime} \nabla^{2} \phi-\phi \nabla^{2} \phi^{\prime}\right) d V=\oiint_{S}\left(\phi^{\prime} \frac{\partial \phi}{\partial n}-\phi \frac{\partial \phi}{\partial n}\right) d S .
$$

Equation B.5 is known as Green's theorem in the second form.

\section{B.2 Green's Theorem for Irrotational Acyclic Flow}

We now wish to consider the motion of a body having surface $S$ in an ideal fluid with an outer boundary surface $\Sigma$. It is assumed that the fluid motion was started from a state of rest or uniform motion so that it is irrotational for all times. We further assume that the flow is acyclic (i.e., $\Gamma=0$ about all closed fluid curves) so that a single-valued solution, $\phi(\vec{r}, t)$, to Laplace's equation must exist.

Recalling Green's theorem in the second form, Equation B.5, let

$$
\phi=\phi(\vec{r}, \mathrm{t}) \text { where } \nabla^{2} \phi=0
$$


and

$$
\phi^{\prime}=\frac{1}{R} \quad \text { where } \quad R=|\vec{r}-\vec{\zeta}|
$$

Here we note that $R$ is simply the distance between a fixed point, $P$, located by $\vec{r}$ and any point in the volume, $v$. Since $\nabla^{2} \phi^{\prime}=0$ except at $\vec{\zeta}=\vec{r}$, an infinitesimal spherical boundary surface, $\sigma$, is placed about $P$ as shown in Figure B.1, thereby excluding it from the volume, v.

Under these conditions, Equation B.5 becomes

$$
0=I_{1}+I_{2}+I_{3}
$$

where

$$
\begin{aligned}
& I_{1}=\oiint_{S}\left[\frac{\partial \phi}{\partial n} \frac{1}{R}-\phi \frac{\partial}{\partial n}\left(\frac{I}{R}\right)\right] d S \\
& I_{2}=\oiint_{\Sigma}\left[\frac{\partial \phi}{\partial n} \frac{1}{R}-\phi \frac{\partial}{\partial n}\left(\frac{1}{R}\right)\right] d S \\
& I_{3}=\oiint_{\sigma}\left[\frac{\partial \phi}{\partial n} \frac{1}{R}-\phi \frac{\partial}{\partial n}\left(\frac{1}{R}\right)\right] d S
\end{aligned}
$$

To determine the potential, $\phi(\vec{r}, t)$, due to the motion of $s$, we consider the limits of $I_{2}$ and $I_{3}$ as $\sum \rightarrow \infty$ and $\sigma \rightarrow 0$, respectively. Equation B.6b may be shown to go to zero as $\Sigma+\infty$, since the fluid at infinity is at rest or in uniform motion and $\phi$ and $\frac{\partial \phi}{\partial n}$ must vanish there. Now consider Equation B.6c. Noting that since $\sigma$ is a spheri- 


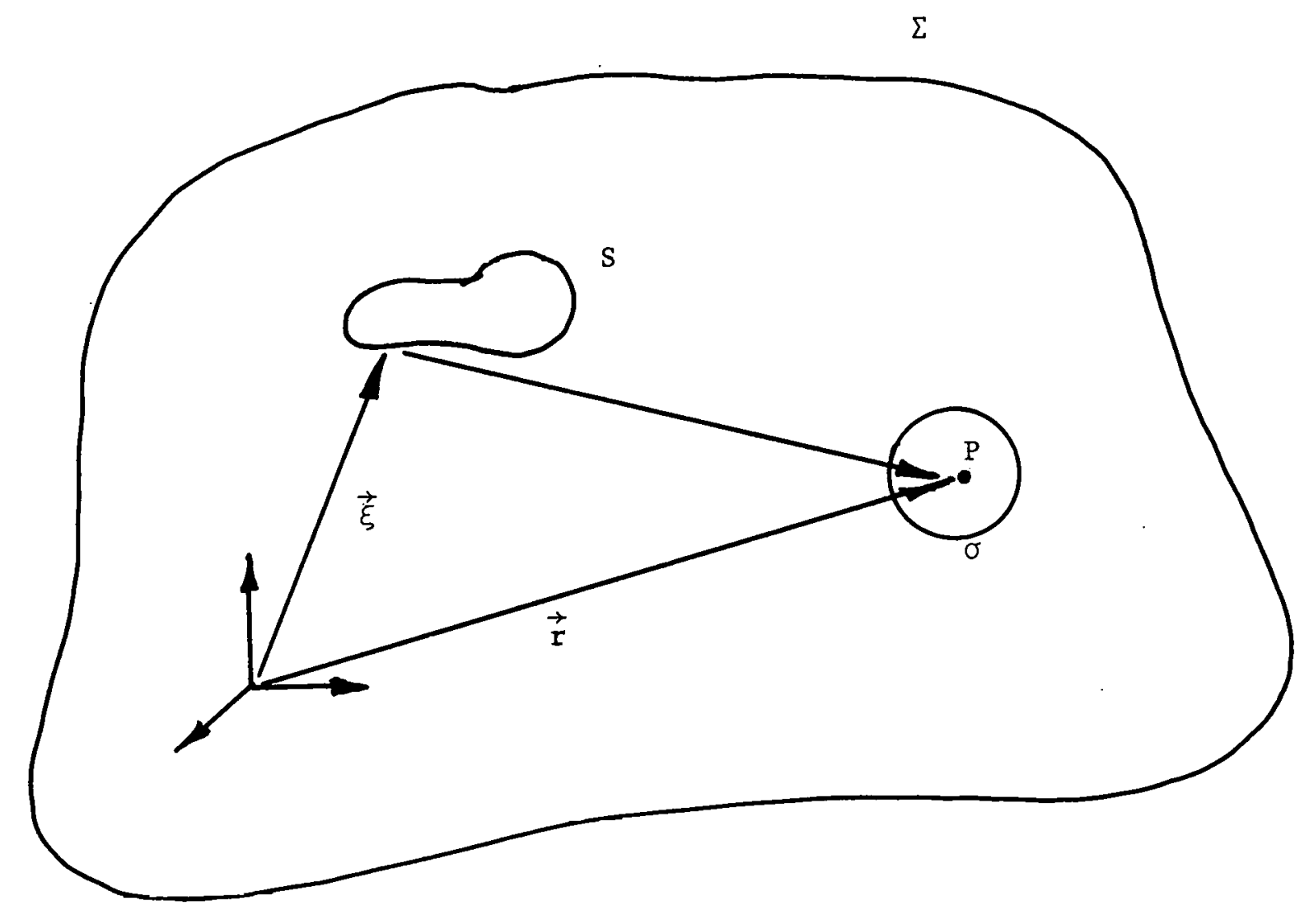

Figure B.1 Intergration Regions for Equation B.6 
cal surface, then $R=\varepsilon$ and $\frac{\partial}{\partial n}=\frac{\partial}{\partial \varepsilon}$, and the equation way be rewritten as

$$
I_{3}=\lim _{\varepsilon \rightarrow 0} \iint_{\sigma}\left[\frac{\partial \phi}{\partial \varepsilon} \frac{1}{\varepsilon}-\phi \frac{\partial}{\partial \varepsilon}\left(\frac{1}{\varepsilon}\right)\right] \varepsilon^{2} \mathrm{~d} \Omega
$$

where $\mathrm{d} s i=$ differential solld angle. By the mean value theorem of integration, it is possible to determine mean surface values of $\phi$ and $\frac{\partial \phi}{\partial n}$ such that Equation B.7 becomec

$$
I_{3}=\left.\lim _{\varepsilon \rightarrow 0} \frac{\partial \phi}{\partial \varepsilon}\right|_{\text {mean }} \oiint_{\sigma} \frac{\varepsilon^{2}}{\varepsilon} \text { li } \lim _{\varepsilon \rightarrow 0} \oint_{\text {mean }} \oiint_{\sigma} \frac{\varepsilon^{2}}{\varepsilon^{2}} d \Omega
$$

or

$$
I_{3}=4 \pi \phi(\vec{r})
$$

From the preceding conclusions, Equation B. 6 becomes.

$$
\phi(\vec{r})=\frac{1}{4 \pi}\left\{-\oiint_{E} \frac{\partial \phi}{\partial n} \frac{1}{R} d S+\oiint_{S} \phi \frac{\partial}{\partial n}\left(\frac{1}{R}\right) d S\right\}
$$

which is the desired integral solution to Laplace's equation. $\Lambda$ notable feature of Equation B.9 is that the potential at any given point in the fluid flow field is determined by the potential and its normal derivative on the bounding surface. In addition, the terms $\frac{1}{4 \pi K}$ and $\frac{\partial}{\partial \mathrm{n}}\left[\frac{1}{4 \pi \mathrm{K}}\right]$ may be recognized as potential sources and doublest, reopoctively.

Although the previous derivacton was given for a threediuensional flow fleld, equivalent relations for a two-dimensional fleld may be determined also. In this case, Gauss' divergence theorem relates surface and line integrals rather than volume and surface integrals. The derivation of Green's theorem proceeds in exactly the same way. The 
result is given by

$$
\iint_{S}\left(\phi^{\prime} \nabla^{2} \phi-\phi \nabla^{2} \phi^{\prime}\right) \mathrm{dS}=\oint_{\mathrm{C}}\left(\phi^{\prime} \frac{\partial \phi}{\partial \mathrm{n}}-\phi \frac{\partial \phi^{\prime}}{\partial \mathrm{n}}\right) \mathrm{d} \ell .
$$

We allow $\phi$ to be the solution of $\nabla^{2} \phi=0$ and choose $\phi=\ln (\mathrm{R})$ where $R=|\vec{r}-\vec{\zeta}|$ as before. Substitution into Equation B. 10 yields

$$
0=\oint_{\sigma+\sum+S}\left[\ln R \frac{\partial \phi}{\partial \mathrm{n}}-\phi \frac{\partial}{\partial \mathrm{n}}(\ln R)\right] \mathrm{d} \ell .
$$

If we take the limits as $\Sigma \rightarrow \infty$ and $\sigma \rightarrow 0$, the two-dimensional equivalent

of Equation $B .9$ results and is given by

$$
\phi(\vec{r})=-\oint_{C}\left[\frac{\ell n R}{2 \pi} \frac{\partial \phi}{\partial n}+\frac{\phi}{2 \pi} \frac{\partial}{\partial n}(\ln R)\right] d l .
$$

For this case, the surfaces are represented by two-dimensional sources and doublets. 
Consider an interior and exterior flow separated by a bounding surface, $S$, as shown in Figure C.l. Let $\phi_{E}(\vec{r}, t)$ be the solution of $\nabla^{2} \phi E$ for the exterior flow and $\phi_{I}(\vec{r}, t)$ be the solution for the interior flow.

Recall that Green's theorem was given by Equation B.5 as

$$
\iiint_{V}\left(\phi^{\prime} \nabla^{2} \phi-\phi \nabla^{2} \phi^{\prime}\right) d V=\oiint_{S}\left(\phi^{\prime} \frac{\partial \phi}{\partial n}-\phi \frac{\partial \phi^{\prime}}{\partial \mathrm{u}}\right) d S,
$$

For the exterior flow, we choose $\phi=\phi_{\mathrm{E}}$ and $\phi_{\mathrm{E}}=\frac{1}{\mathrm{R}}$ where $\mathrm{k}=\left|\overrightarrow{\mathrm{r}}-\overrightarrow{\mathrm{r}}_{\mathrm{E}}\right|$. Now $\nabla^{2} \phi_{\mathrm{E}}=0$ everywhere except at $\vec{\zeta}=\overrightarrow{\mathrm{r}} \cdot$. Consequently, by surrounding the point $P$ at $\vec{r}$ with a spherical boundary and taking the limit as the sphere is reduced to $P$, we find that (as shown in Appendix B)

$$
\phi(\vec{r}, t)=\frac{-1}{4 \pi} \oiint_{S}\left[\frac{\partial \phi_{E}}{\partial n_{E}} \frac{1}{R}-\phi_{E} \frac{\partial}{\partial n}\left(\frac{1}{R}\right)\right] d S .
$$

For the interior flow, $\phi=\phi I$, and again, $\phi I=\frac{1}{R}$. Since we are only concerned with the polnts $P$ exterior to $S, R=\left|\vec{r}-\vec{\zeta}_{1}\right|$ cannot be zero and $\nabla^{2} \phi_{I}=0$ for a11 $\vec{\zeta}_{\mathrm{T}}$. Equation $\mathrm{C} .1$ for the interlor problem may be written as

$$
0=\frac{-1}{4 \pi} \oiint_{S}\left[\frac{\partial \phi_{I}}{\partial n_{I}} \frac{1}{R}-\phi_{\underline{I}} \frac{\partial}{\partial n_{I}}\left(\frac{I}{R}\right)\right] d S
$$

Adding the exterior and interior solutions, Equations C.2 and C.3, respectively, yields

$$
\begin{aligned}
\phi(\vec{r}, t) & \left.=\frac{-1}{4 \pi} \oiint_{S}\left(\frac{\partial \phi_{E}}{\partial \underline{n}_{E}}+\frac{\partial \phi_{I}}{\partial n_{1}}\right) \frac{1}{R}\right] d S \\
& +\frac{1}{4 \pi} \oiint_{S}\left[\phi_{E} \frac{\partial}{\partial n_{E}}\left(\frac{1}{R}\right)+\phi_{I} \frac{\partial}{\partial n_{I}}\left(\frac{1}{R}\right)\right] d S
\end{aligned}
$$


Noting that $\frac{\partial}{\partial n_{E}}=-\frac{\partial}{\partial n_{I}}$, Equation $C .4$ may be rewritten as

$$
\begin{aligned}
\phi(\vec{r}, t) & =\frac{-1}{4 \pi} \iint_{S}\left[\left(\frac{\partial \phi_{E}}{\partial n_{E}}-\frac{\partial \phi_{I}}{\partial n_{E}}\right) \frac{1}{R}\right] d S \\
& +\frac{1}{4 \pi} \iint_{S}\left[\left(\phi_{E}-\phi_{I}\right) \frac{\partial}{\partial n_{E}}\left(\frac{1}{R}\right)\right] d S .
\end{aligned}
$$

From Equation C.5, it is noted that the external potential field is again determined by surface distributions of potential sources and doublets. In this case, however, it is clear that the strength distributions of the singularities is dependent on the interfor flow. Suppose that the interior flow is such that $\phi_{\mathrm{I}}=\phi_{\mathrm{E}}$ on $\mathrm{S}$, thereby requiring a continuous tangential velocity across $S$, with the possibility of a discontinuous normal velocity left open. Equation C.5 reduces to

$$
\phi(\vec{r}, t)=\frac{-1}{4 \pi} \iint_{S}\left[\left(\frac{\partial \phi_{E}}{\partial n_{E}}-\frac{\partial \phi_{I}}{\partial n_{E}}\right) \frac{1}{R}\right] d S
$$

illustrating that the potential field may be represented by a surface distribution of sources.

Suppose that $\frac{\partial \phi_{E}}{\partial n_{E}}=\frac{\partial \phi_{I}}{\partial n_{E}}$ so tlial the normal velocity across $S$ is continuous while the tangential velocity may be discontinuous. Equation C. 5 reduces to

$$
\phi(\vec{r}, t)=\frac{1}{4 \pi} \iint_{S}\left[\left(\phi_{E}-\phi_{I}\right) \frac{\partial}{\partial n_{E}}\left(\frac{1}{R}\right)\right] d S
$$

which provides an expression for $\phi(\vec{r}, t)$ with a surface distribution of doublets.

We may generalize the preceding discussion by stating that a solu- 
tion to Laplace's equation may be uniquely represented by surface distributions of

1) sources only

2) doublets only

3) sources and doublets on separated portions of the surface

4) linearly related distributions of sources and doublets.

As a final comment, it is noted that doublet distribulions are

requlfed on lifting eurfaces, since only they allow the possibllity of discontinuous tangential velucities and finite circulations. 


\section{APPENDIX D}

EQUIVALENT DOUBLET AND VORTEX SURFACE DISTRIBUTIONS

The objective for this appendix is to demonstrate the equivalence of surface distributions of potential doublets and vortices. This will be accomplished by first deriving the expression for the potential of a three-dimensional doublet. The velocity field induced by a vortex segment will then be derived, and from that the velocity potential induced by a ring vortex will be determined. It will then be clear that the potential field due to a distribution of uniform strength doublets over an open surface is equivalent to that induced by a line vortex around the boundary of the surface with strength equal to that of the doublets. Finally, the general conclusion will be drawn that any surface distribution of doublets may be represented by a distribution of vortices oriented normal to the gradient of doublet strength and having strengths equal to that gradient.

\section{D.1 The Potential Doublet}

A potential doublet may be formed by taking the limit as a potential source and sink are brought together where the axis of the doublet is along the line connecting source and sink, centers as shown in Figure D.1. This is expressed by

$$
\begin{aligned}
& \left.\underset{\text { doublet }}{\phi(\vec{r})}=\underset{\delta s \rightarrow 0}{\lim _{\text {source }}^{\phi(\vec{r})}}+\phi(\vec{r}+\delta \vec{s})\right] \\
& =\lim _{\delta s \rightarrow 0}[\underset{\text { source }}{\phi(\vec{r})}-\phi(\vec{r}+\delta \vec{s})]
\end{aligned}
$$

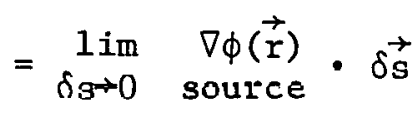




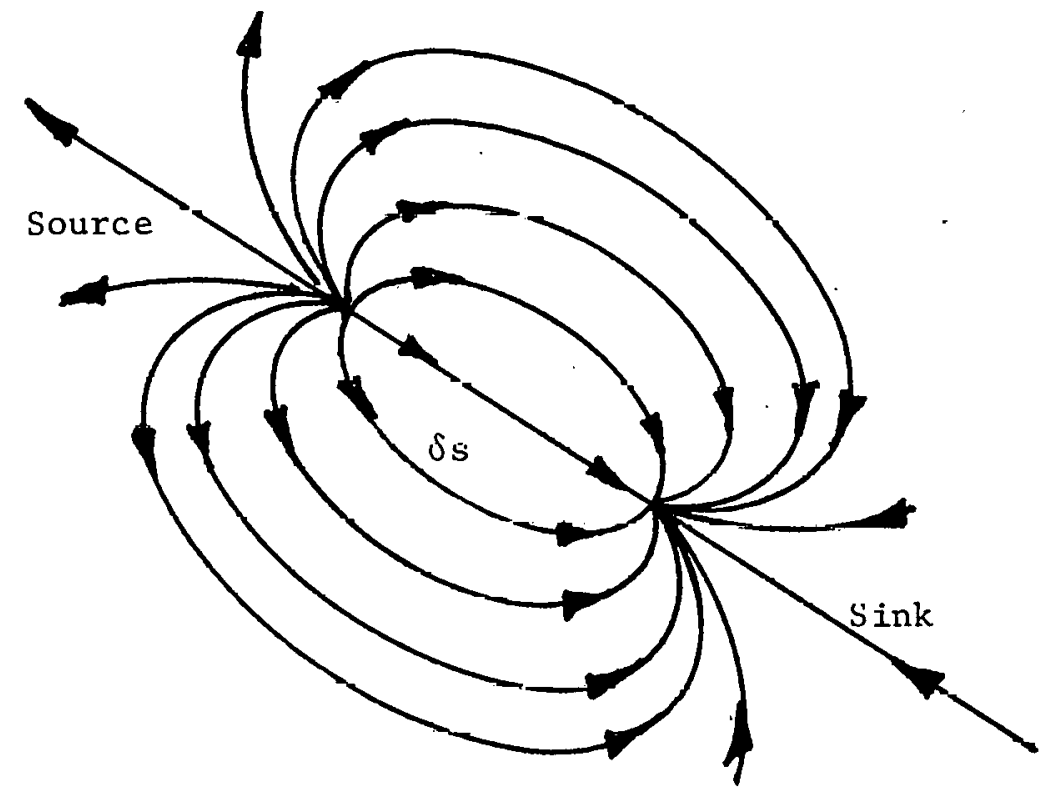

Figure D.1 Dnıhlet. as a Combined Source and Sink Flow 


$$
\begin{aligned}
& =\underset{\text { source }}{\nabla \phi(\vec{r})} \cdot \vec{e}_{s} \\
& =\frac{\mu}{4 \pi} \nabla\left(\frac{1}{R}\right) \cdot \vec{e}_{s}
\end{aligned}
$$

where

$$
R=|\vec{r}-\vec{\zeta}|
$$

From Equation D. 1, the potential field due to a surface distribution of doublets is given by

$$
\phi(\vec{r})=\iint_{S} \frac{\mu}{4 \pi} \nabla\left(\frac{1}{R}\right) \cdot \vec{n} d S
$$

where $\overrightarrow{\mathrm{n}}=$ the surface normal.

D.2 Velocity Field Induced by a Vortex Segment

For an ideal fluid, the incompressibility requirement may be stated as

$$
\nabla \cdot \vec{U}=0
$$

Since for all vector fields, $A$, it ie truc that

$$
\nabla \cdot(\nabla \times \vec{A})=0
$$

we may choose

$$
\overrightarrow{\mathrm{U}}=\nabla \times \overrightarrow{\mathrm{A}}
$$

Given $U_{1}$, the vorticity fleld is given by

$$
\vec{\Omega}=\nabla \times \nabla \times \vec{A}
$$


or

$$
\vec{\Omega}=\nabla(\nabla \cdot \vec{A})-\nabla^{2} \vec{A}
$$

Since $\vec{A}$, to this point, is indeterminate to the extent of the gradient of a vector, we may further stipulate that

$$
\nabla \cdot \vec{A}=0
$$

With Equation D.5, Equation $D .4$ may be rewritten as

$$
\nabla^{2} \vec{A}=-\vec{\Omega}
$$

This is Poisson's equation for $\vec{A}$. By solving for $\vec{A}$, It will then be possible to determine the velocity field utilizing Equation D. 3.

Consider the solution of the vector relationship, Equation D.6, which has the following component. equations:

$$
\begin{aligned}
& \nabla^{2} A_{x}=-3 i_{x} \\
& \nabla^{2} \Lambda_{y}=-5 i_{y} \\
& \nabla^{2} A_{z}=-\Omega_{z}
\end{aligned}
$$

It is sufficient to determine the solution of C.7a over a.11 space, keeping in mind that $\bar{\delta}$ and $\vec{A}$ must vanish at inf1nity.

Recall that Green's theorem was given by Equation B.5 as

$$
\iiint_{V}\left(\phi^{\prime} \nabla^{2} \psi-\phi^{2} \nabla^{\prime}\right) d V=\iint_{S}\left(\phi^{\prime} \frac{\partial \phi}{\partial \boldsymbol{I}}-\frac{\partial \phi^{\prime}}{\partial \mathrm{n}}\right) \mathrm{dS}
$$

For $\phi$ and $\phi^{\prime}$, choose

$$
\begin{aligned}
\phi & =A_{x} \\
\phi^{\prime} & =1 / R
\end{aligned}
$$


where

$$
R=\left|\vec{r}-\vec{r}_{1}\right|
$$

Therefore,

$$
\begin{aligned}
\nabla \phi & =\nabla A_{x} \\
\nabla^{2} & =\nabla^{2} A_{x} \\
\nabla^{2} \phi & =-\Omega_{x} \\
\nabla \phi^{\prime} & =\vec{R} / R^{3} \\
\nabla \phi^{\prime} & =0 \text { for } \overrightarrow{\mathrm{r}} \neq \overrightarrow{\mathrm{r}}_{1} \\
& =\infty \text { for } \overrightarrow{\mathrm{r}}=\overrightarrow{\mathrm{r}}_{1}
\end{aligned}
$$

Substitution of Equations D.9 - D.11 into Equation D.8 yields

$$
\begin{aligned}
& \iiint_{V} \frac{\Omega x}{R} d V=-\sum^{1 i m} \iint_{\Sigma}\left(A_{x} \nabla\left(\frac{1}{R}\right)+\frac{\nabla A}{R}\right) \cdot \vec{n} d S \\
& -\lim _{\sigma \rightarrow \infty} \iint_{\sigma}\left(A_{x} \nabla\left(\frac{1}{R}\right)+\frac{\nabla A_{x}}{R} \cdot \vec{n} d S\right.
\end{aligned}
$$

where $\Sigma=$ outer boundary

$$
\sigma=\text { spherical shell about singular point at } \overrightarrow{\mathbf{r}}_{1}=\overrightarrow{\mathrm{r}} \text {. }
$$

The surfaces $\Sigma$ and $\sigma$, as well as the vectors $\vec{r}, \vec{r}_{1}$ and $\vec{R}$ are illustrated in Figure D.2. 


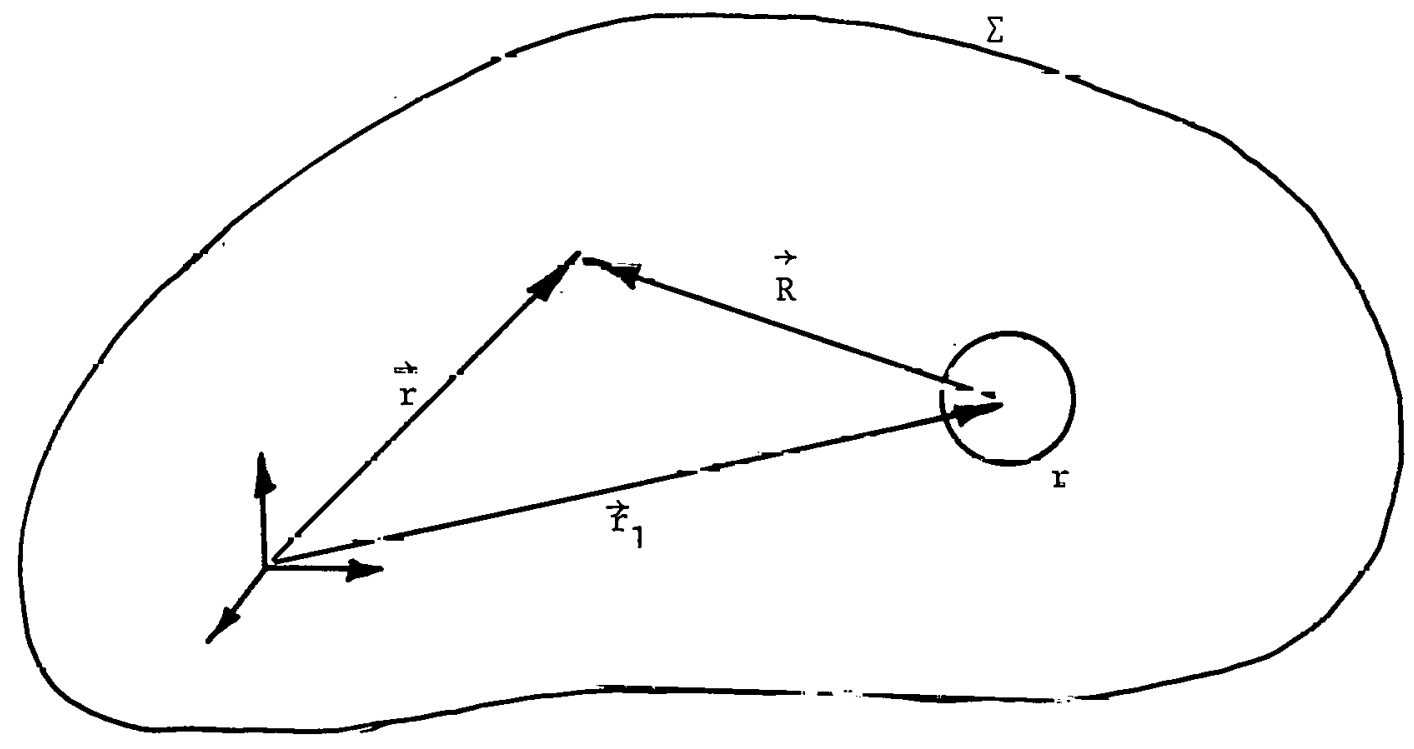

Figure D.2 Integration Rég1ons fui Equation D.12 
If we consider the first integral of the right hand side of Equation D. 12 and allow $\Sigma$ to be a spherical surface which spreads to Infinity in the limit, it follows thal

$$
\left.0=\lim _{\mathrm{R} \rightarrow \infty} \iint_{\Sigma}\left(\frac{-\mathrm{A}}{\mathrm{R}^{2}}\right)+\frac{1}{\mathrm{R}} \frac{\sigma \mathrm{A}}{\sigma \mathrm{R}}\right) \mathrm{R}^{2} \mathrm{~d} \Omega
$$

where $\mathrm{d} \Omega=$ differential solfd angle and it is assumed that

$$
\lim _{\mathrm{R} \rightarrow \infty} \frac{\mathrm{R}}{\sigma \mathrm{A}_{\mathrm{x}} / \sigma \mathrm{R}}=0
$$

For the second integral over $\sigma$,

$$
\begin{gathered}
I_{2}=\lim _{R \rightarrow 0} \iint_{\sigma}\left(\frac{-A}{R^{2}}+\frac{1}{R} \frac{\sigma A}{\sigma R}\right) R^{2} d \Omega \\
I_{2}=\lim _{R \rightarrow 0}\left(-\left.A_{x}\right|_{\text {mean }} \iint_{\sigma} \mathrm{d} \Omega\right. \\
+\left.\frac{\sigma A}{\sigma R}\right|_{\text {mean }} \iint_{\sigma} \mathrm{d} \Omega \\
I_{2}=-4 \pi A_{x}\left(\vec{r}_{1}\right)
\end{gathered}
$$

Substitution of Equations D.13 and D.14 into Equation D.12 yields

$$
A_{X}(\vec{r})=\frac{1}{4 \pi} \quad \iiint_{V} \frac{\Omega_{X}\left(\vec{r}_{1}\right)}{R} d V
$$

When the equivalent solutions for Equation $\mathrm{D.} 7 \mathrm{~b}$ and $\mathrm{c}$ are determined, we may write

$$
\vec{A}(\vec{r})=\frac{1}{4} \cdot \iiint_{V} \frac{\vec{\Omega}\left(\vec{r}_{1}\right)}{R} d V
$$

Now from Equations D. 3 and D.16, we may determine the velocity 
field resulting in the vorticity distribution, $\overrightarrow{\vec{k}}(\overrightarrow{\mathrm{r}})$;

$$
\vec{U}=\nabla_{r} \times \quad A \vec{A}=\frac{1}{4}\left(\nabla_{r} \times \iiint_{V} \frac{\vec{S}\left(\vec{r}_{1}\right)}{R} d V\right.
$$

We now wish to determine the velocity field induced by an infinitesimal vortex filament with strength $\Gamma$ as shown in Figure D.3. From Equation D.16, the incremental contribution to $\vec{A}$ from the filament may be written as

$$
\begin{aligned}
\delta \vec{A}(\vec{r}) & =\frac{1}{4 \pi} \frac{\vec{\Omega}\left(\vec{r}_{1}\right)}{R} d V \\
& -\frac{1}{4 \pi} \cdot \frac{\vec{\Omega}\left(\overrightarrow{\vec{r}}_{1}\right)}{R} \overrightarrow{\mathrm{n}} \mathrm{dS} \cdot d \overrightarrow{\mathrm{D}} .
\end{aligned}
$$

We may write

$$
\mathrm{d} \vec{l}=\frac{\vec{\Omega}}{\Omega} \mathrm{d} l
$$

and

$$
\vec{\Omega} \cdot \vec{n} \text { dS }=\Gamma
$$

so that Equation D. 18 may be rewritten as

$$
\delta \vec{A}(\vec{r})=\frac{\Gamma}{4 \pi} \frac{d \vec{\ell}}{R}
$$

Consèquencly, Llie Llikiémental velocity from tho vortex filament is

$$
\begin{aligned}
\delta \vec{U}(\vec{r}) & =\frac{\Gamma}{\pi} \times \frac{d \vec{l}}{R} \\
& =\frac{\Gamma}{4 \pi} d \vec{l} \times \nabla_{r}\left(\frac{1}{R}\right)
\end{aligned}
$$

where $\vec{d} l$ and $\vec{r}_{1}$ are fixed and $\nabla_{\mathrm{r}}$ indicates that the curl is to be 


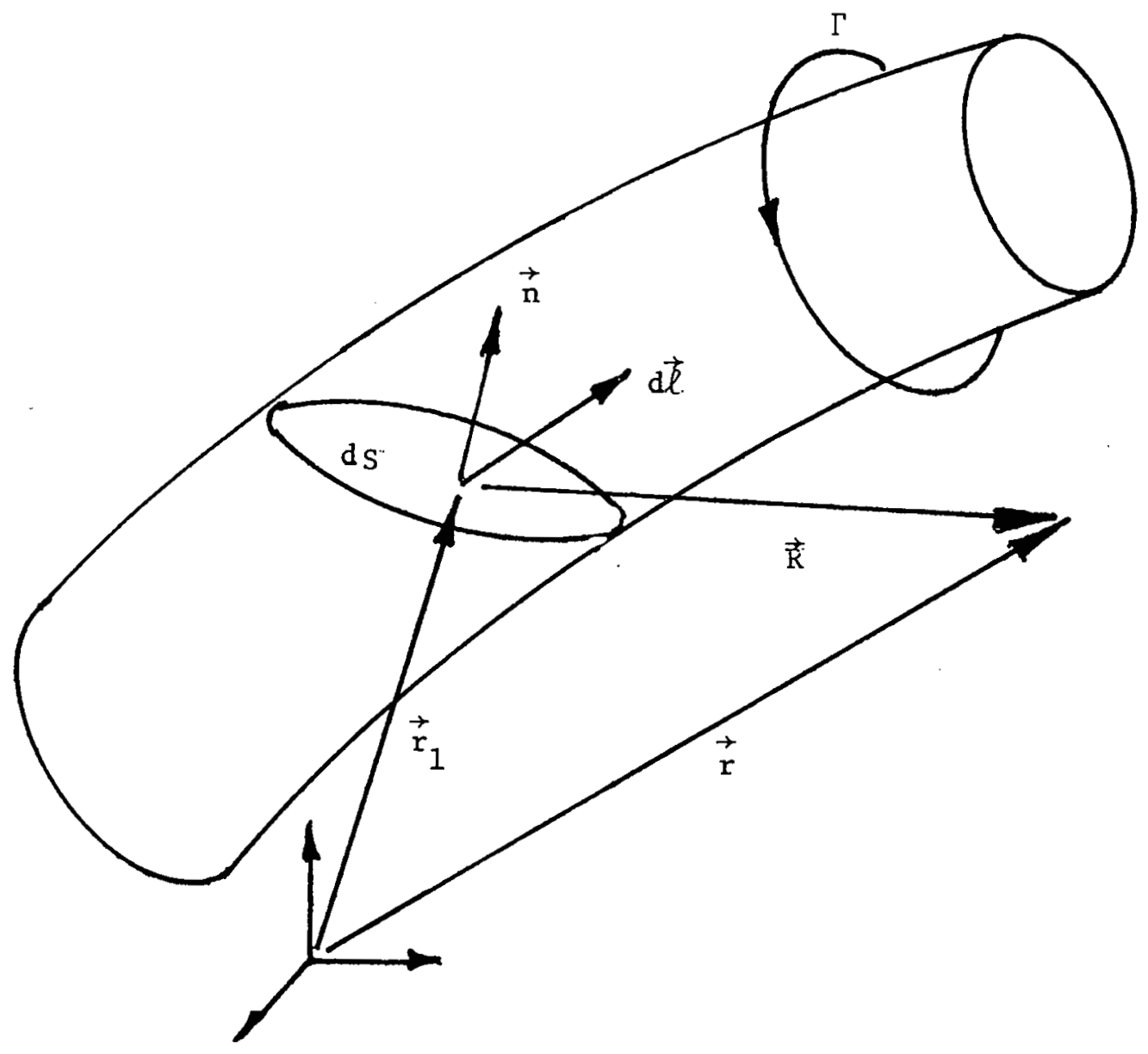

Figure D.3 Veloc1ty Induced by a Vortex Line 
taken with respect to the coordinates of the point $\vec{r}$.

Finally, the velocity induced by a vortex segment is

$$
\overrightarrow{\mathrm{U}}(\overrightarrow{\mathrm{r}})=\frac{\Gamma}{4 \|^{\prime}} \int_{\mathrm{C}} \mathrm{d} \overrightarrow{\mathrm{l}} \times \nabla\left(\frac{1}{\mathrm{R}}\right)
$$

which is the well known Biot-Savart law.

\section{3 Velocity Field of a Vortex Ring}

We would now like to determine the velocity field induced by a vortex ring. Equation D.21 may be written in component form for a closed vortex ring as

$$
\begin{aligned}
& U_{x}(\vec{r})=\frac{\Gamma}{4 \pi} \int_{c}\left[\frac{\sigma}{\sigma z}\left(\frac{1}{R}\right) d l_{y}-\frac{\sigma}{\sigma y}\left(\frac{1}{R}\right) d l_{z}\right] \\
& V_{y}(\vec{r})=\frac{\Gamma}{4 \pi} \int_{c}\left[\frac{\sigma}{\sigma x}\left(\frac{1}{R}\right) d l_{z}-\frac{\sigma}{\sigma z}\left(\frac{1}{R}\right) d l_{x}\right] \\
& U_{z}(\vec{r})=\frac{\Gamma}{4 \pi} \int_{c}\left[\frac{\sigma}{\sigma y}\left(\frac{1}{R}\right) d l_{x}-\frac{\sigma}{\sigma x}\left(\frac{1}{R}\right) d l_{y}\right]
\end{aligned}
$$

By Stokes' theorem, Equation D.22a may be rewritten ao

$$
\mathrm{U}_{\mathrm{x}}(\overrightarrow{\mathrm{r}})=\int_{\mathrm{S}} \vec{\zeta} \cdot \mathrm{d} \vec{\ell}=\iint_{S}(\nabla \times \vec{\zeta}) \cdot \overrightarrow{\mathrm{n}} \mathrm{d} S
$$

where

$$
\vec{\zeta}=\frac{\Gamma}{4 ! 1}\left[\frac{\sigma}{\sigma_{z}}\left(\frac{1}{R}\right) \vec{e}_{y}-\frac{\sigma}{\sigma_{y}}\left(\frac{1}{R}\right) \vec{e}_{z}\right]
$$

By expanding Equation D.23, we tind thà

$$
\begin{aligned}
U_{x}(\vec{r}) & =\frac{\Gamma}{4 \pi} \iint_{S}\left[\left(-\frac{\sigma^{2}}{\sigma y^{2}}-\frac{\sigma^{2}}{\sigma z^{2}}\right) \frac{1}{R} e_{x}+\frac{\sigma^{2}}{\sigma x \sigma y}\left(\frac{1}{R}\right) \vec{e}_{y}\right. \\
& \left.+\frac{\sigma^{2}}{\sigma x \sigma z}\left(\frac{1}{R}\right) \vec{e}_{z}\right] \cdot \vec{n} d S \\
& =\frac{\Gamma}{4 \pi} \iint_{S} \nabla\left(\frac{\sigma}{\sigma x} \frac{1}{R}\right) \cdot \vec{n} d S
\end{aligned}
$$


Similarly, for the other two component equations;

$$
\begin{aligned}
& U_{y}(\vec{r})=\frac{\Gamma}{4 \pi} \iint_{S} \nabla\left(\frac{\sigma}{\sigma y} \frac{1}{R}\right) \cdot \vec{n} d S \\
& U_{z}(\vec{r})=\frac{\Gamma}{4 \pi} \iint_{S} \nabla\left(\frac{\sigma}{\sigma z} \frac{1}{R}\right) \cdot \vec{n} d S
\end{aligned}
$$

We conclude that

$$
\overrightarrow{\mathrm{U}}(\overrightarrow{\mathrm{r}})=\frac{\Gamma}{4 \pi} \iint_{\mathrm{S}} \nabla\left[\nabla\left(\frac{1}{\mathrm{R}}\right) \cdot \overrightarrow{\mathrm{n}}\right] \mathrm{dS}
$$

and

$$
\phi(\vec{r})=\frac{\Gamma}{4 \pi} \iint_{S} \nabla\left(\frac{1}{R}\right) \cdot \vec{n} \mathrm{dS}
$$

\section{D.4 Equivalency of Doublet and Vortex Distributions}

To summarize, in Section D.1, the potential due to an isolated doublet was derived. Following that, the potential due to a surface distribution of doublets was given by Equation D.2. For the special cases of distribution of uniform strength doublets, the potential becomes

$$
\phi(\vec{r})=\frac{\mu}{4 \pi} \iint_{S} \nabla\left(\frac{1}{R}\right) \cdot \vec{n} d S
$$

where $n=u(s)$, the surface normal and

$$
R=|\vec{r}-\vec{\xi}|
$$


In sections D. 2 and D. 3 , the velocity potential due to a vortex ring was determined by deriving the velocity field due to an arbitrary distribution of vorticity and specializing it for the case of an isolated vortex filament. The resulting potential field of a vortex ring given by Equation D.2 and is repeated here;

$$
\phi(\vec{r})=\frac{\Gamma}{i \pi l} \iint_{S} \nabla\left(\frac{1}{R}\right) \cdot \vec{n} \mathrm{dS}
$$

where $\mathrm{S}$ is any open surface bounded by the vortex ring.

Comparison of fiquations D.27 and D, 28 revealo that a vortex ring of strength $\Gamma$ is equivalent to a distribution of doublets with strengths $\mu=\Gamma$ over any arbitrar1ly shaped surface bounded by the vortex ring. If two constant strength doublet surfaces share a coinmon boundary, as shown in Figure D.4, it follows that the potential for that segment of the boundary is equivalent to a vortex segment with a strength equal to the difference of the doublet strengths. This idea may be carried further by envisioning any surface as being made up of infinitesimal areas of constant strength doublets. In the limit, as the areas are reduced to points, the equivalent vortex representation would be a vortex sheet with the filaments directed normal to the gradient of doublet strength and filament clrculations equal to the gradient. 


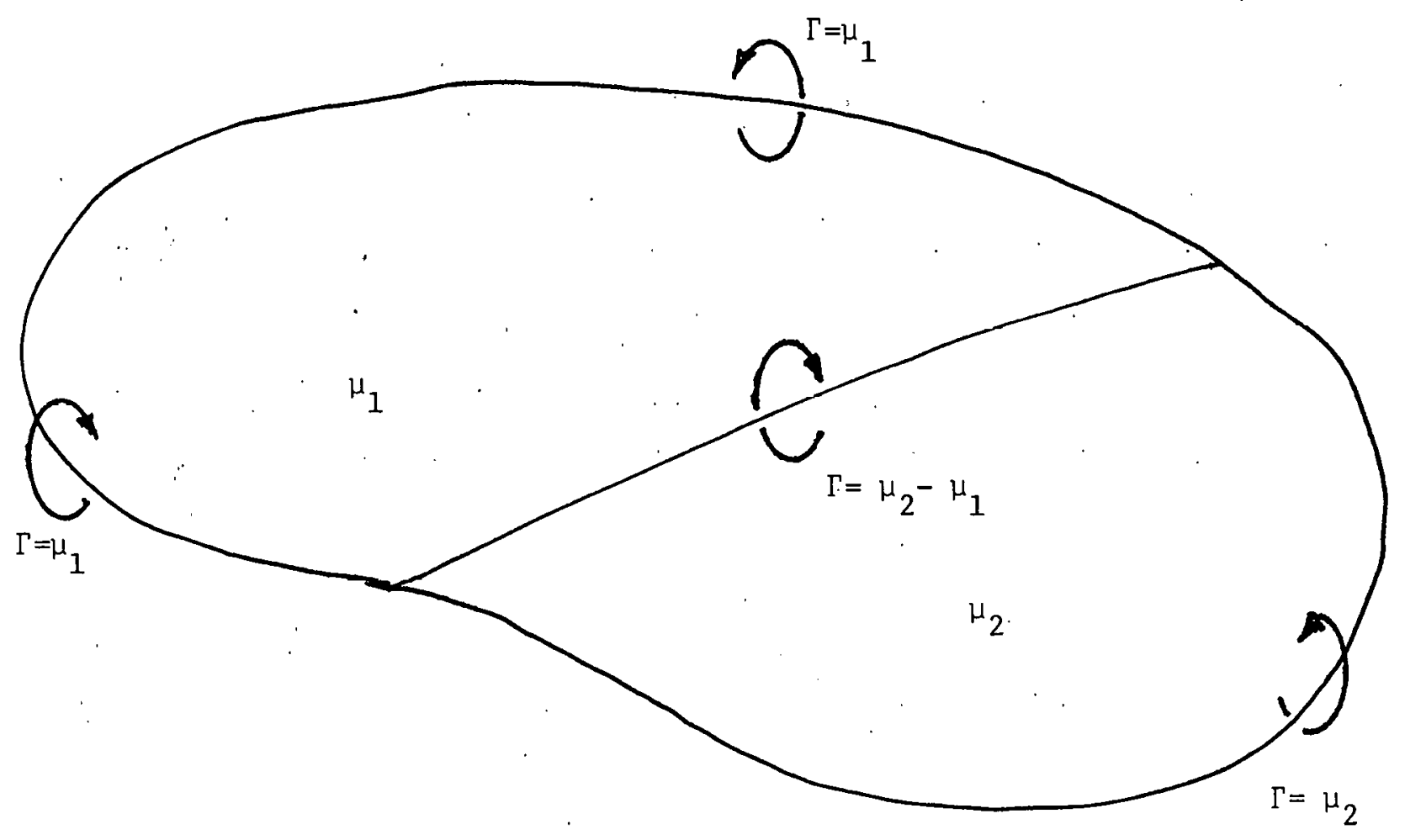

Figure D.4 Vortex Representation of Uniform Strength Doublet Panels 
APPENDIX E

EXPERIMENTAL PRESSURE DATA

In this appendix typical plots of pressure data are given. These plots consist of pressure coefficient curves at selected blade positions in the second revolution of the rotor. Data for the three tip-to-windspeed ratios of $2.5,5.1$, and 7.6 are given In Figures E.1, E.2, and E.3 respectively. 

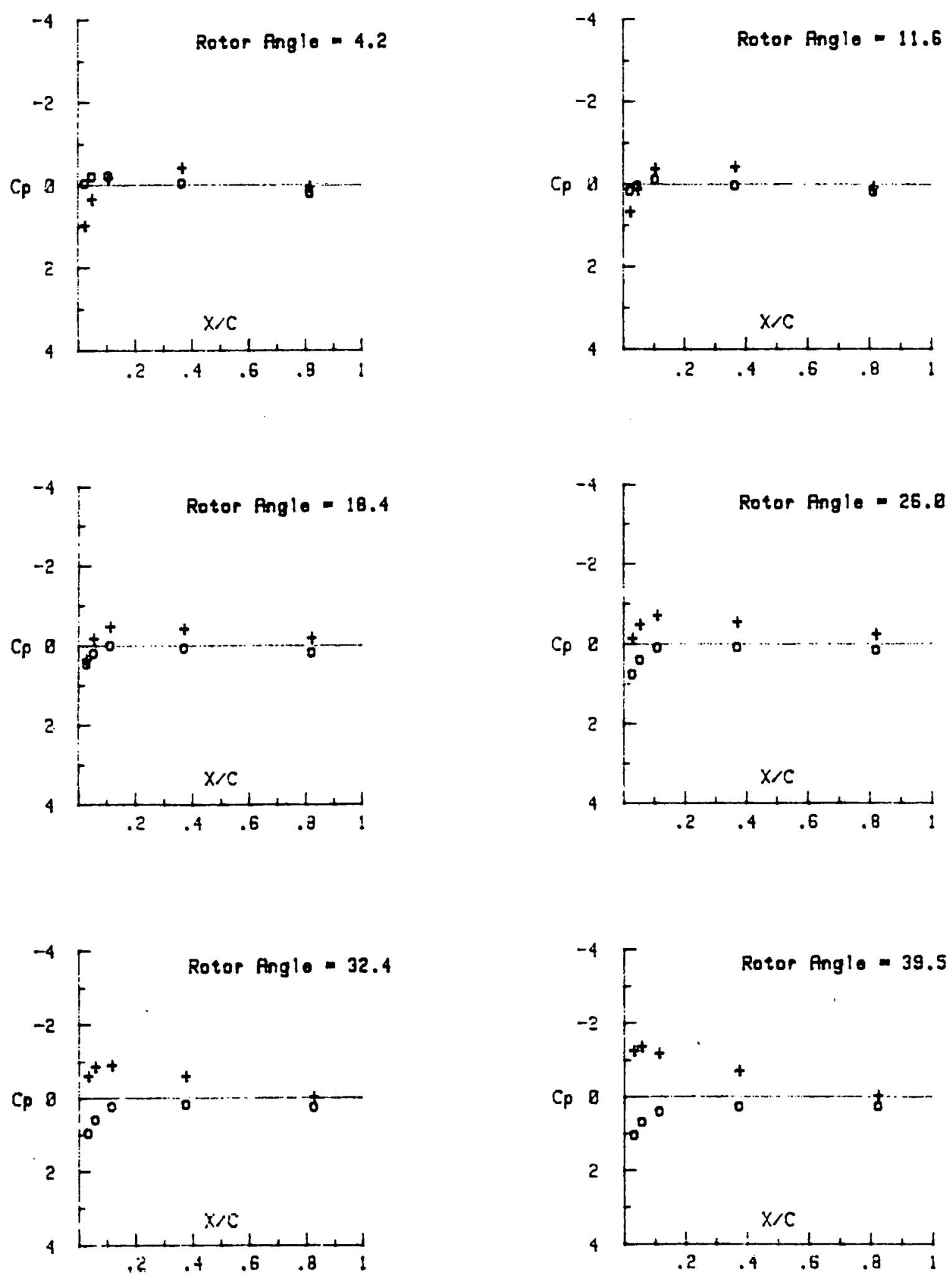

Figure E.1: Effect of Rotor Angle on $C_{p}$ Profiles $T S R=2.5, C / R=0.25, N B=1$, Outer Surface $-\circ$, Inner Surface - + 

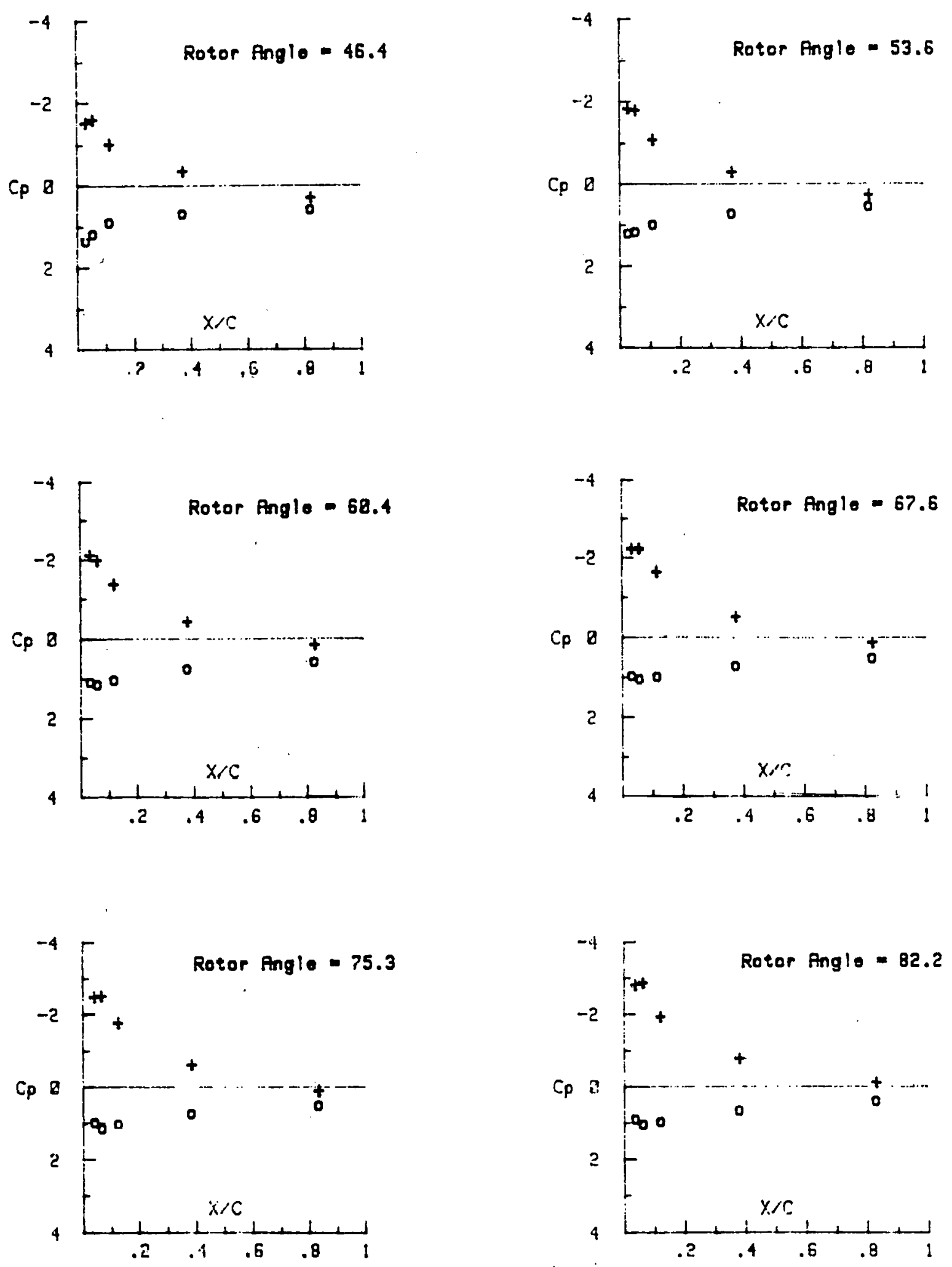

Figure E.1: Continued 

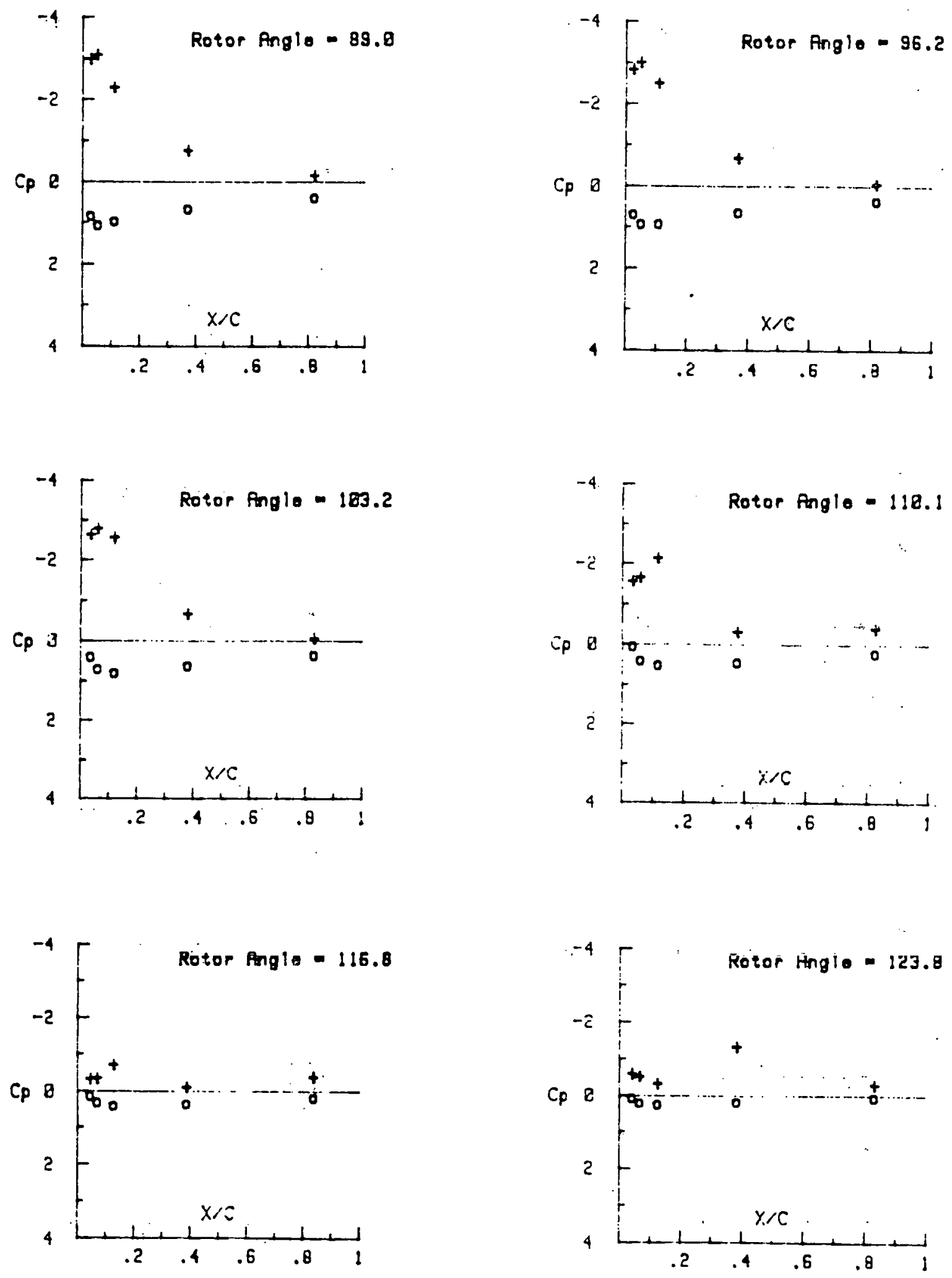

Figure E.1: Continued 

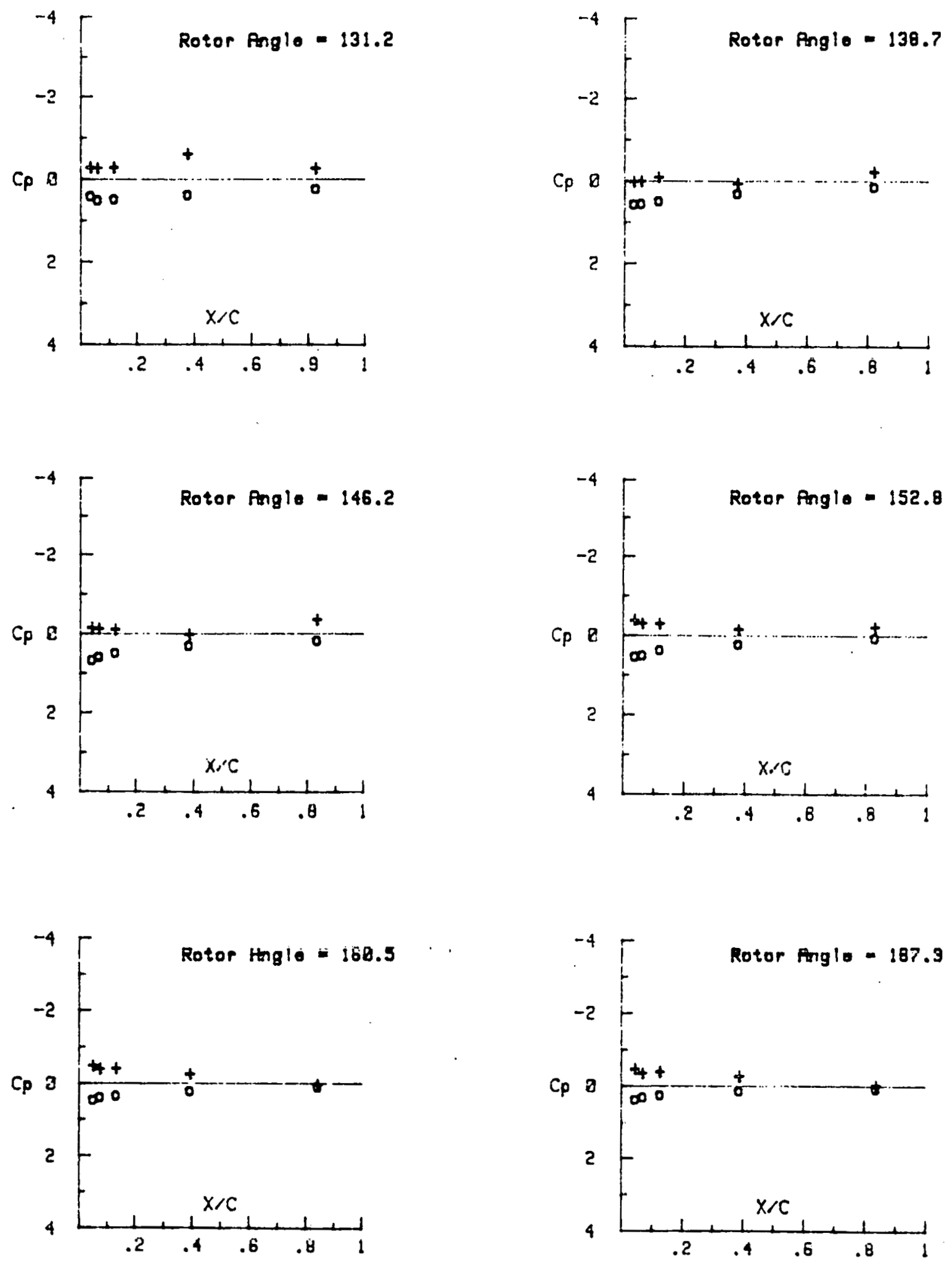

Figure E.1: Continued 

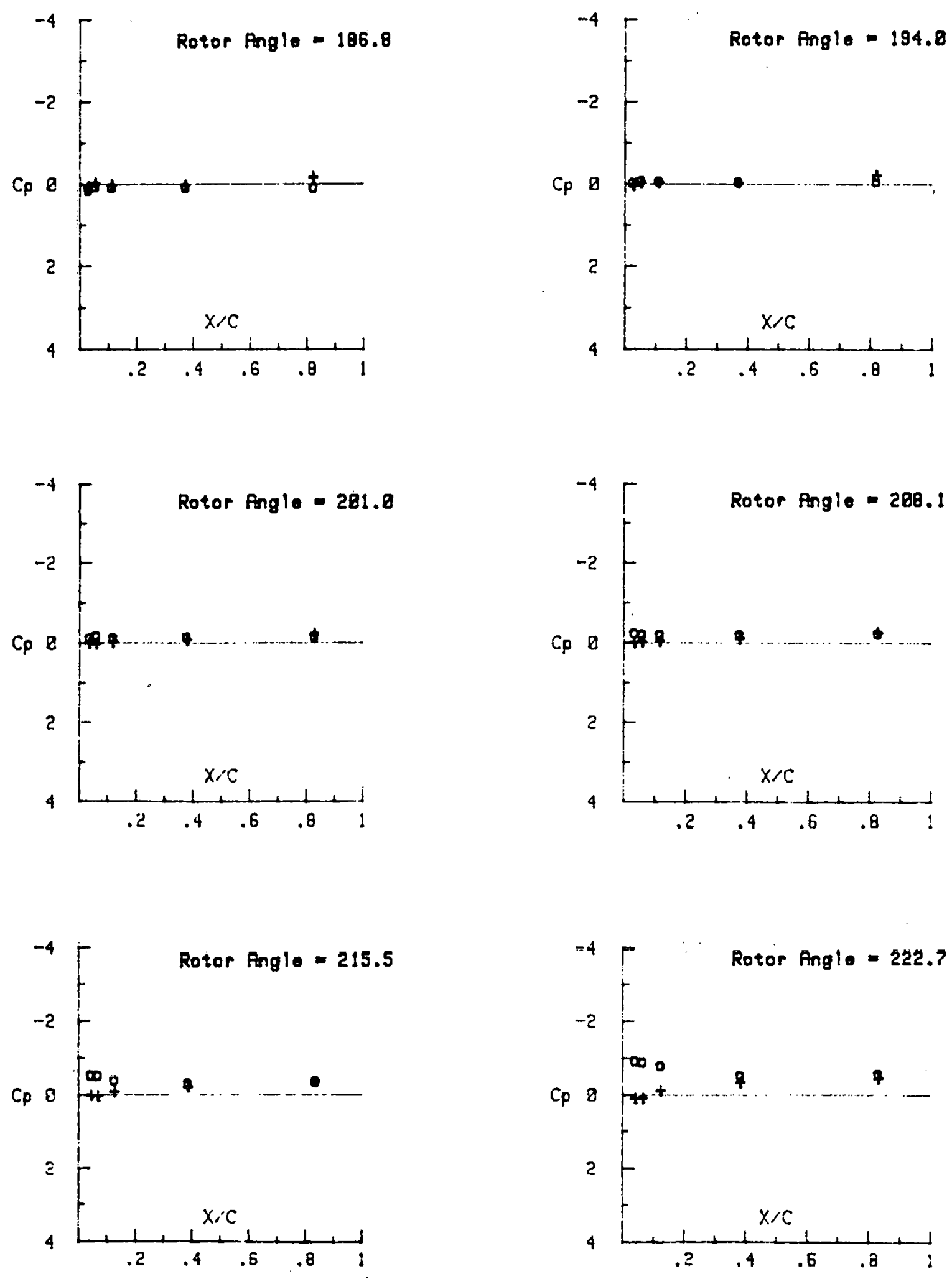

Figure E.1: Continued 

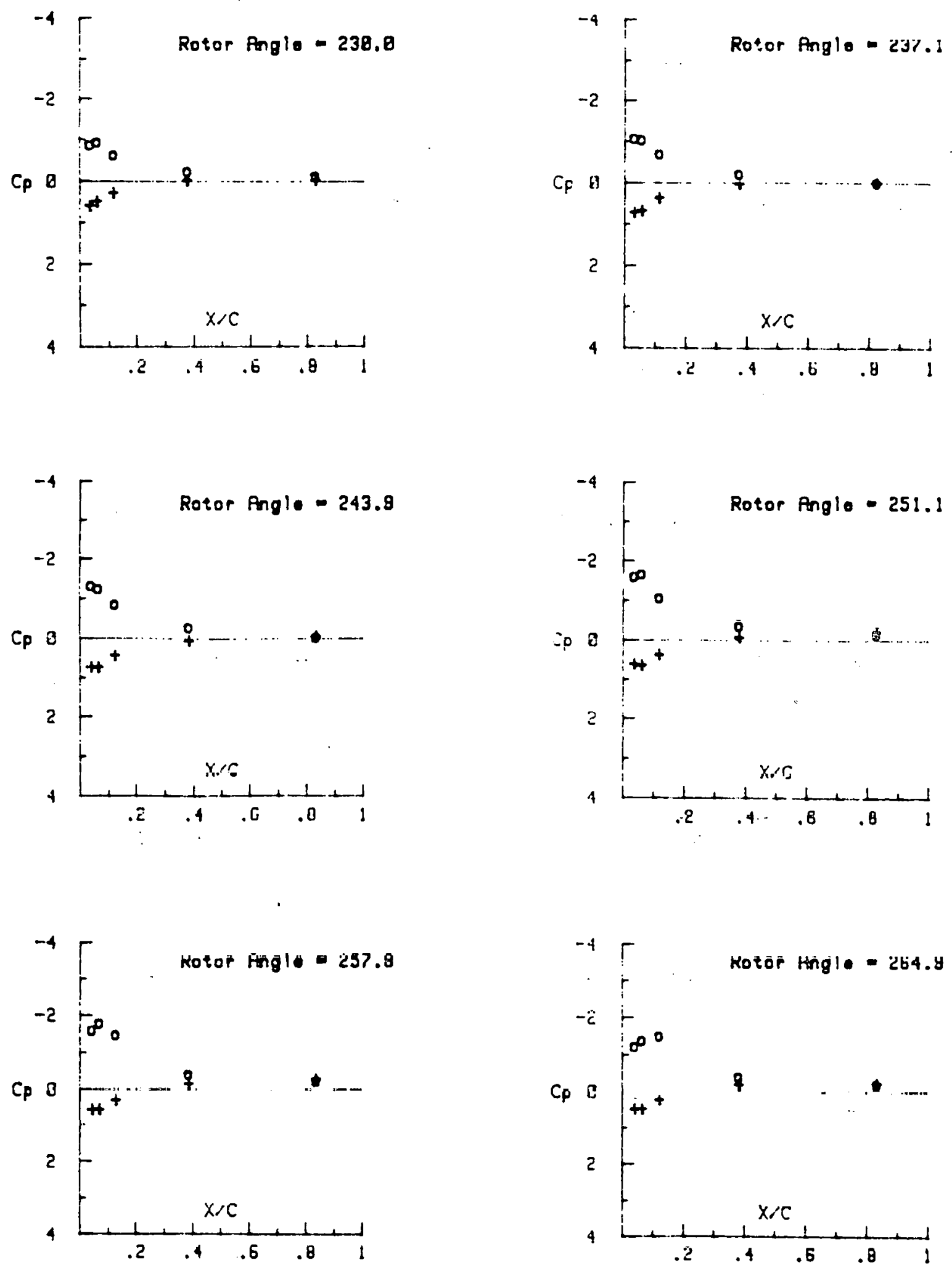

Figure E.1: Continued 

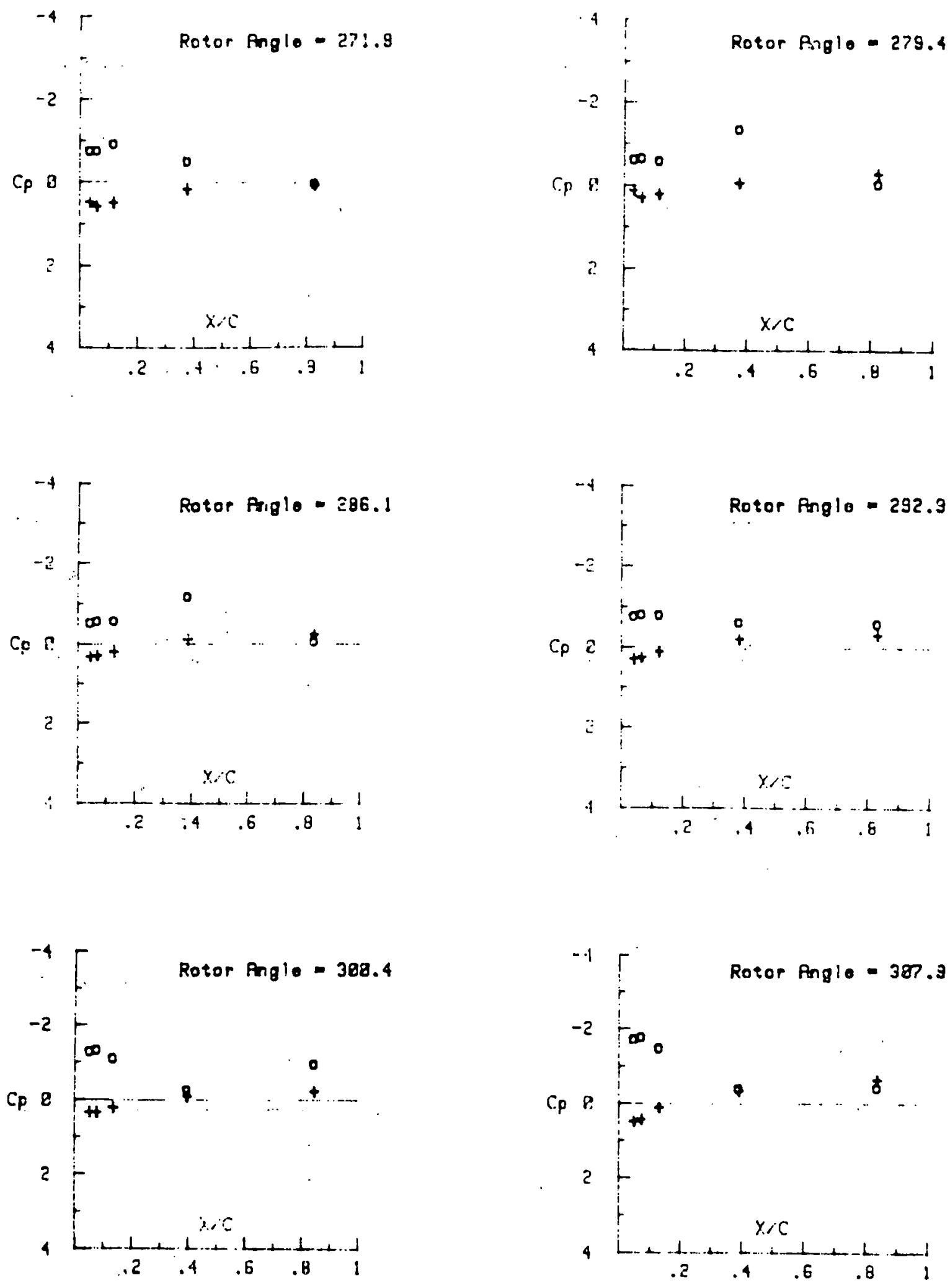

Figure E.1: Continued 

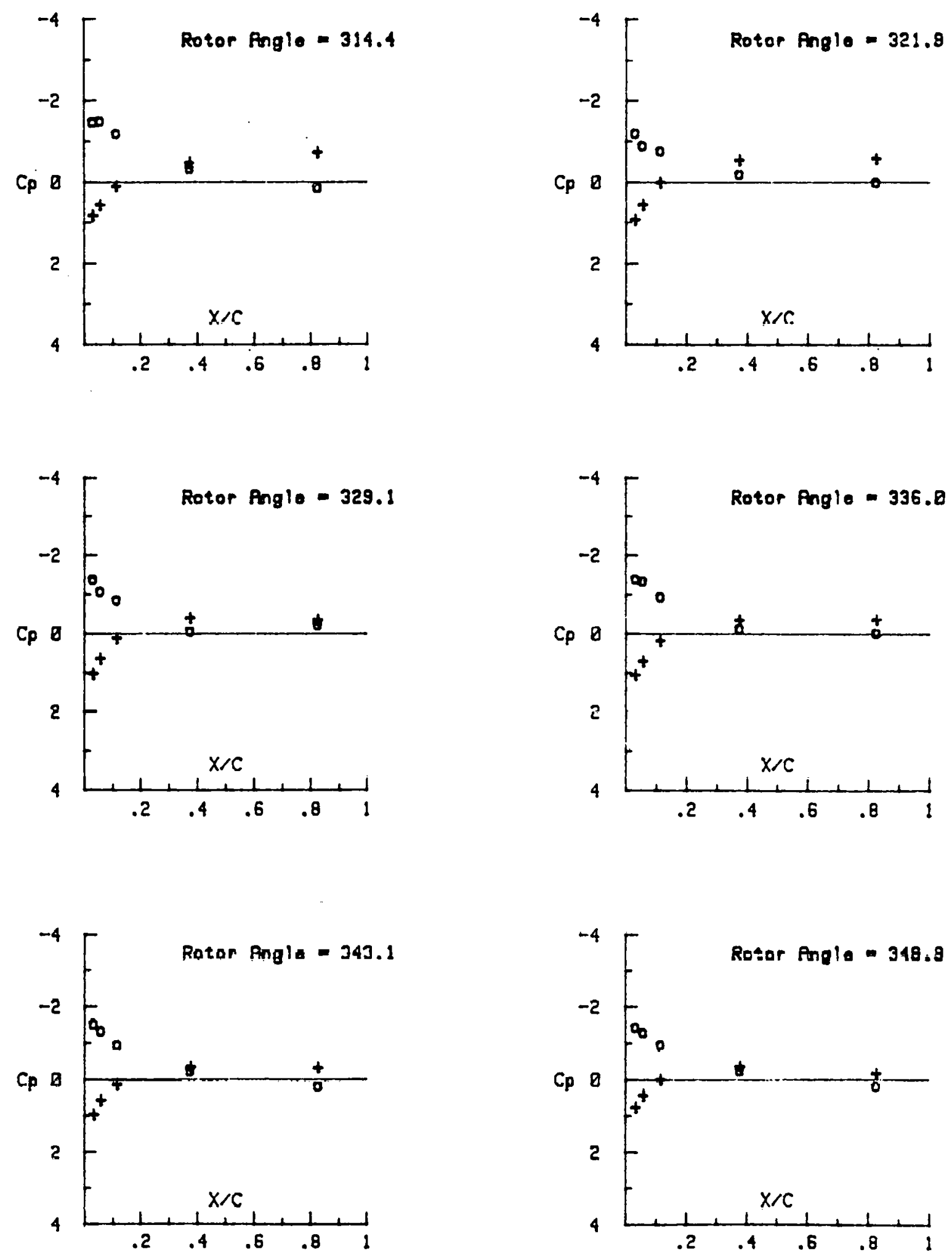

Figure E.1: Continued 

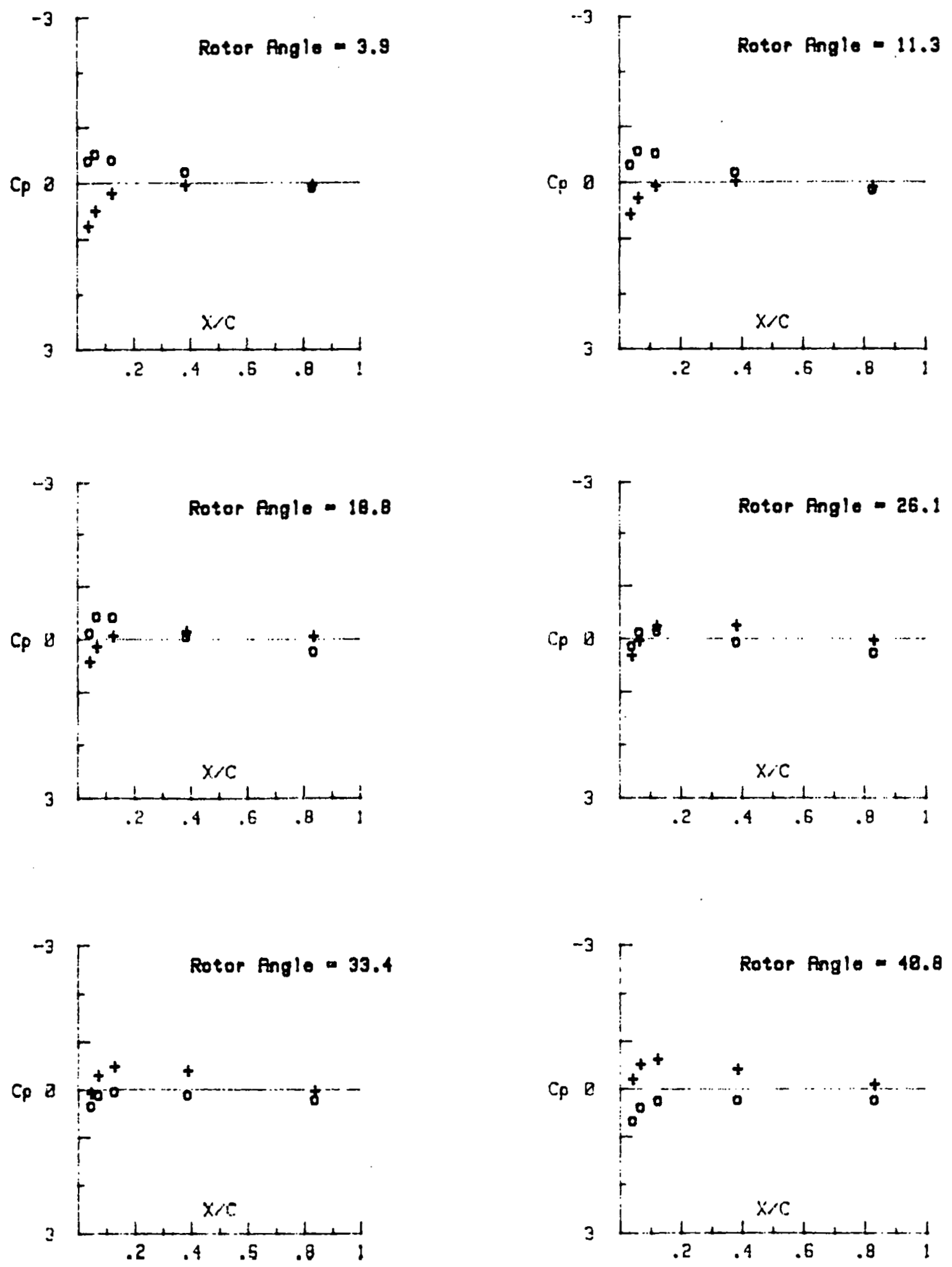

Fig. E.2: Effect of Rotor Angle on $C_{p}$ Profiles

$\left(T S R=5.1, C / R=0.25, \mathrm{NB}^{\mathrm{p}}=1\right.$, Outcr Surface 0 , Inner Surface + ) 

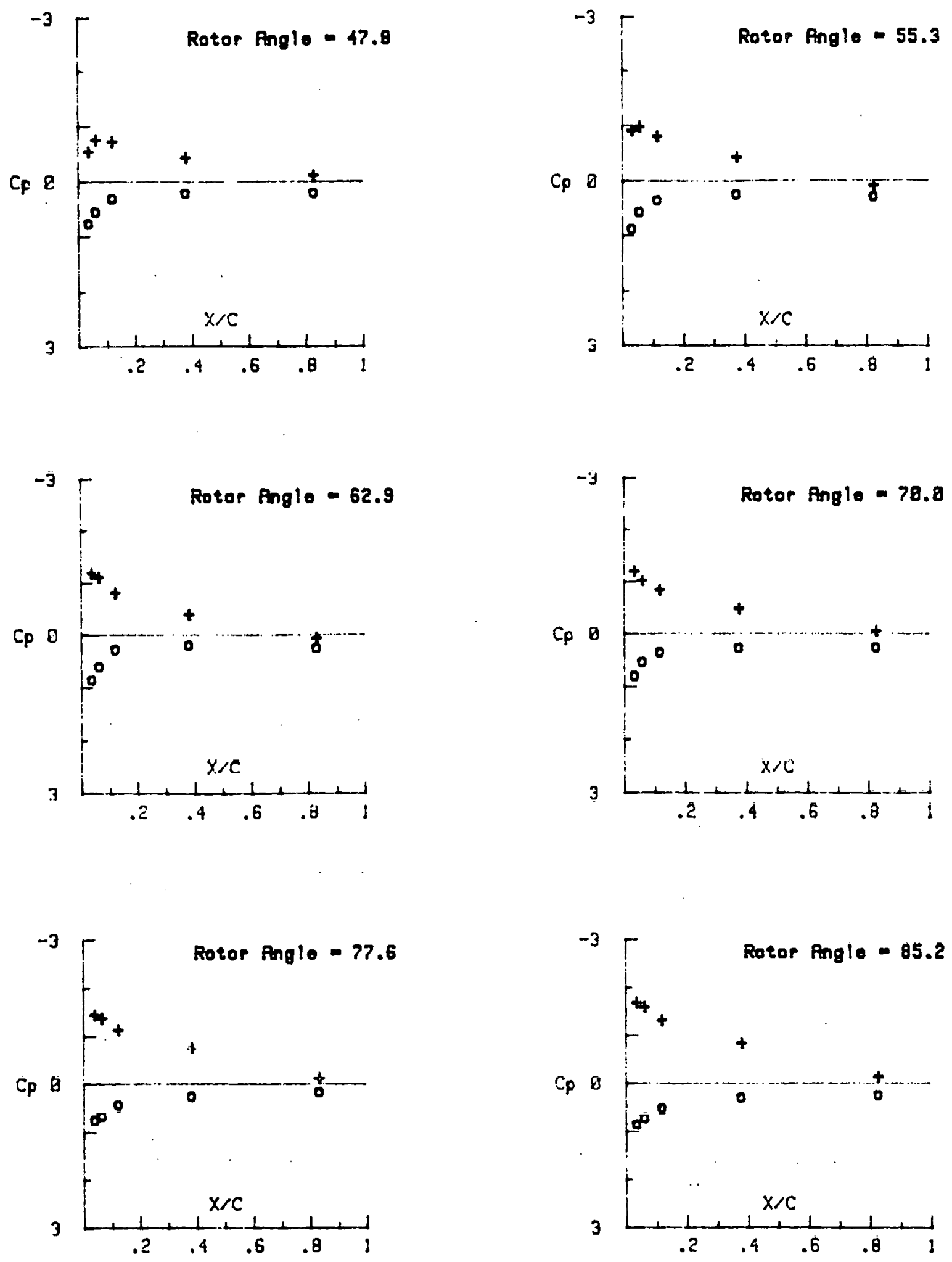

Figure E.2: Continued 

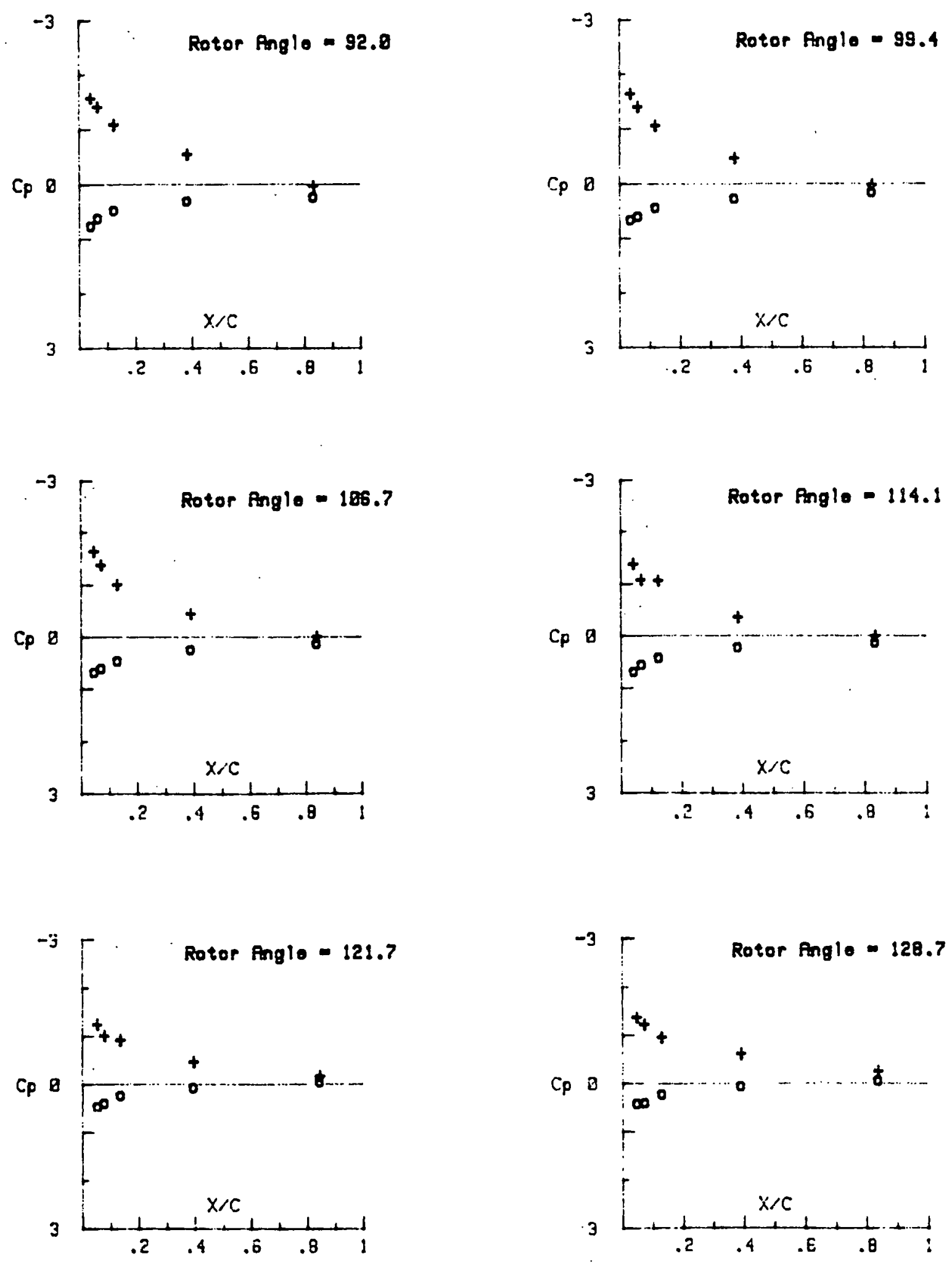

Figure E.2: Continued 

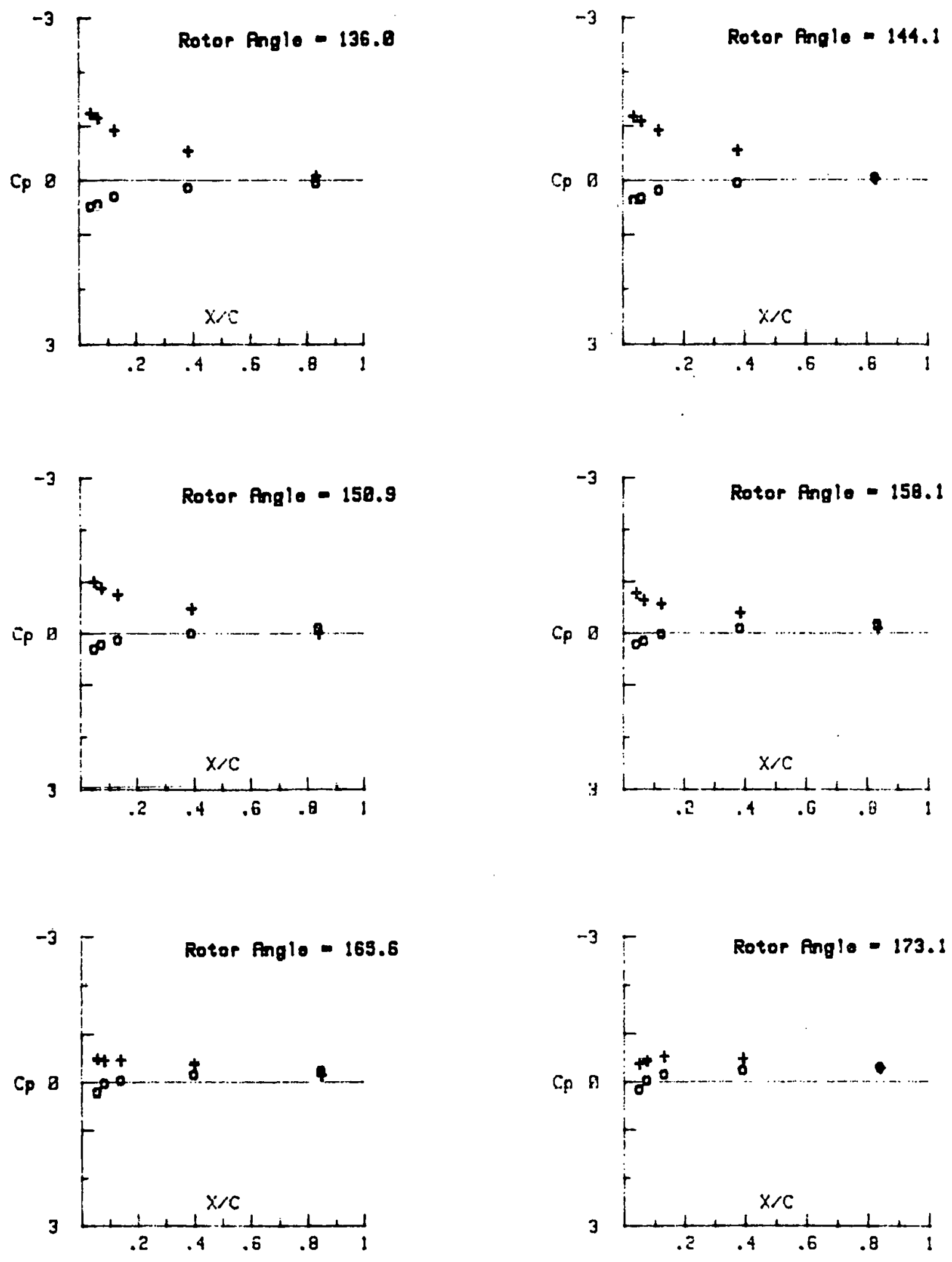

Figure E.2: Continued 

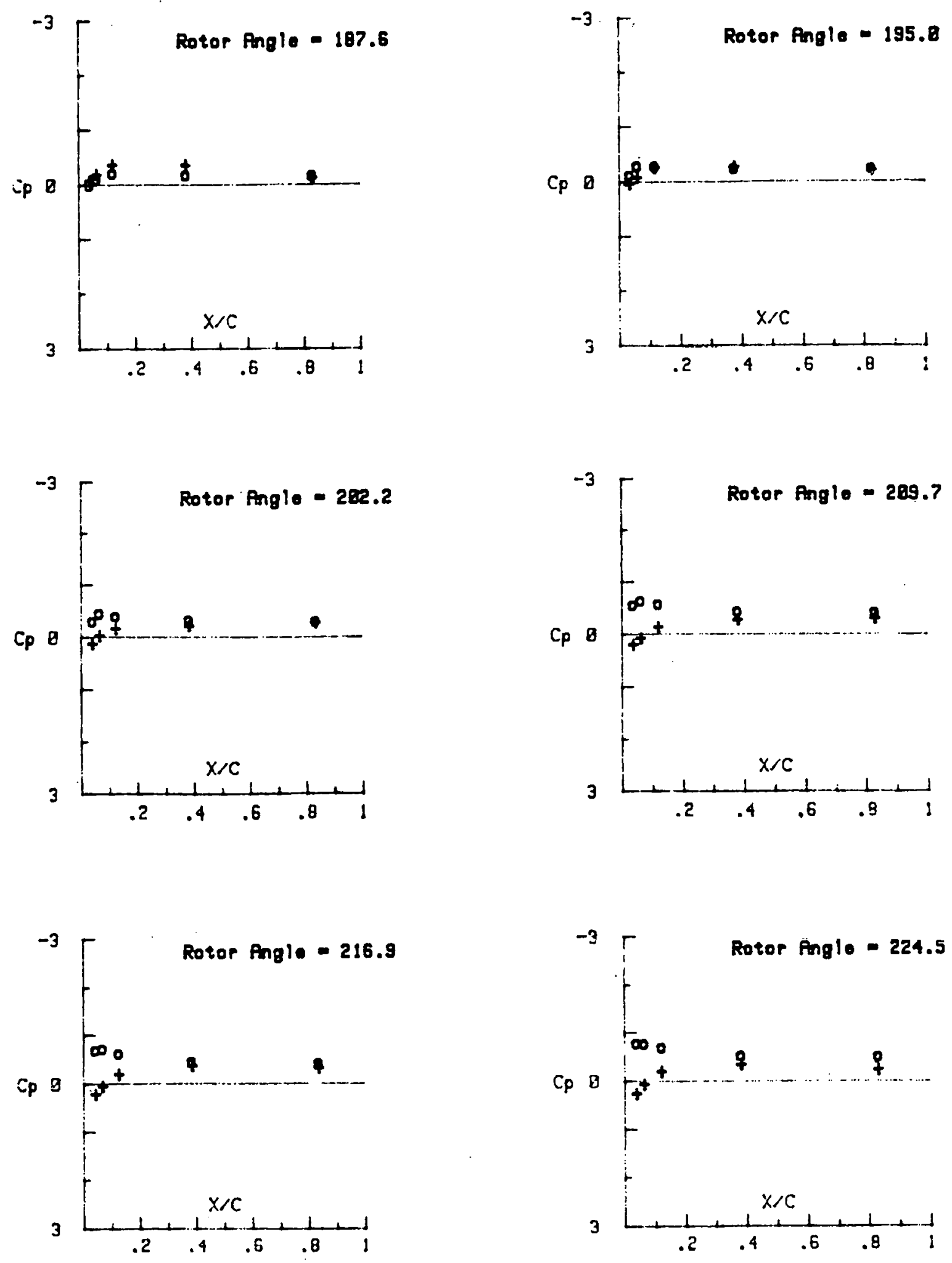

Figure E.2: Continued 

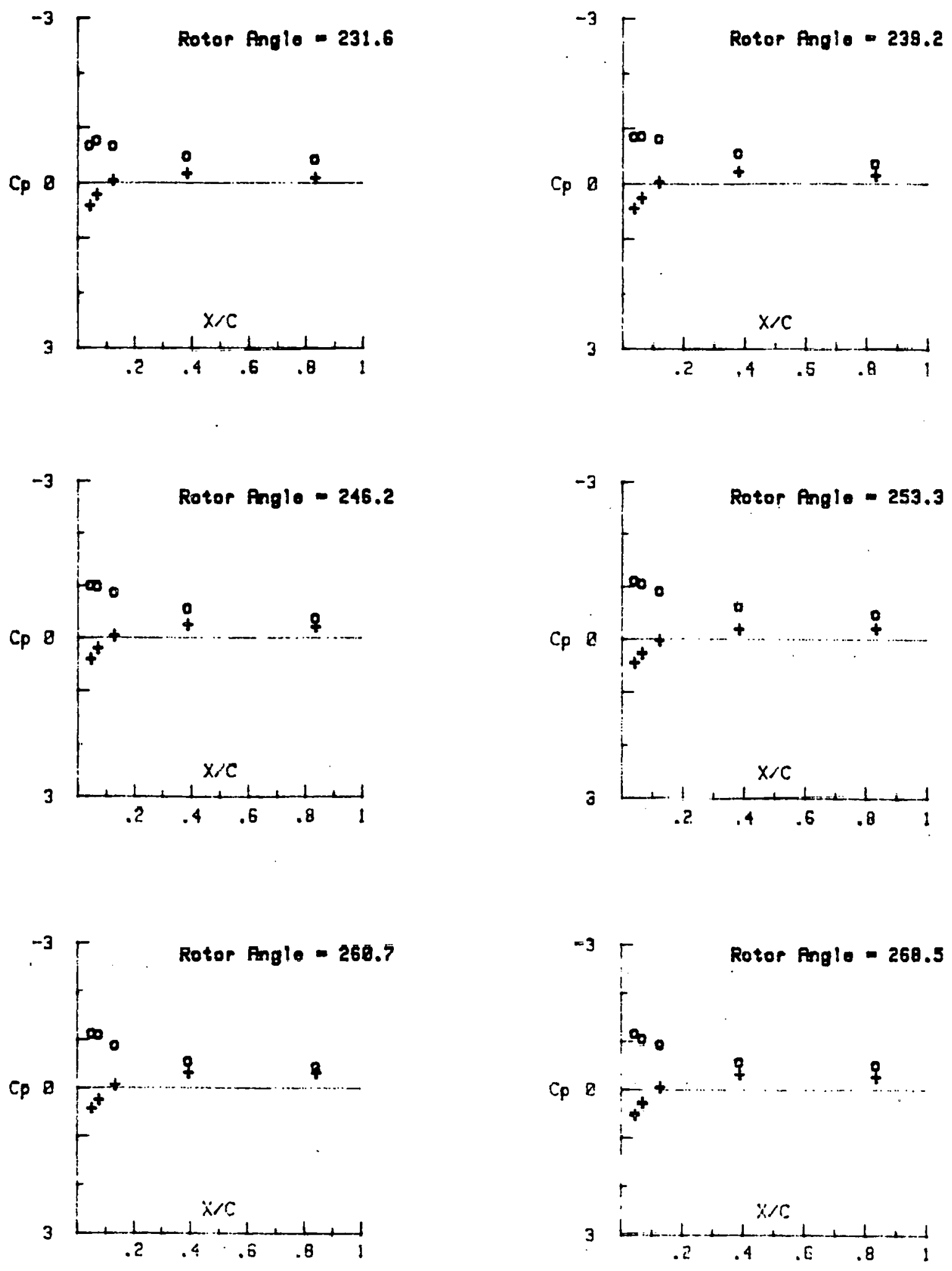

Figure E.2: Continued 

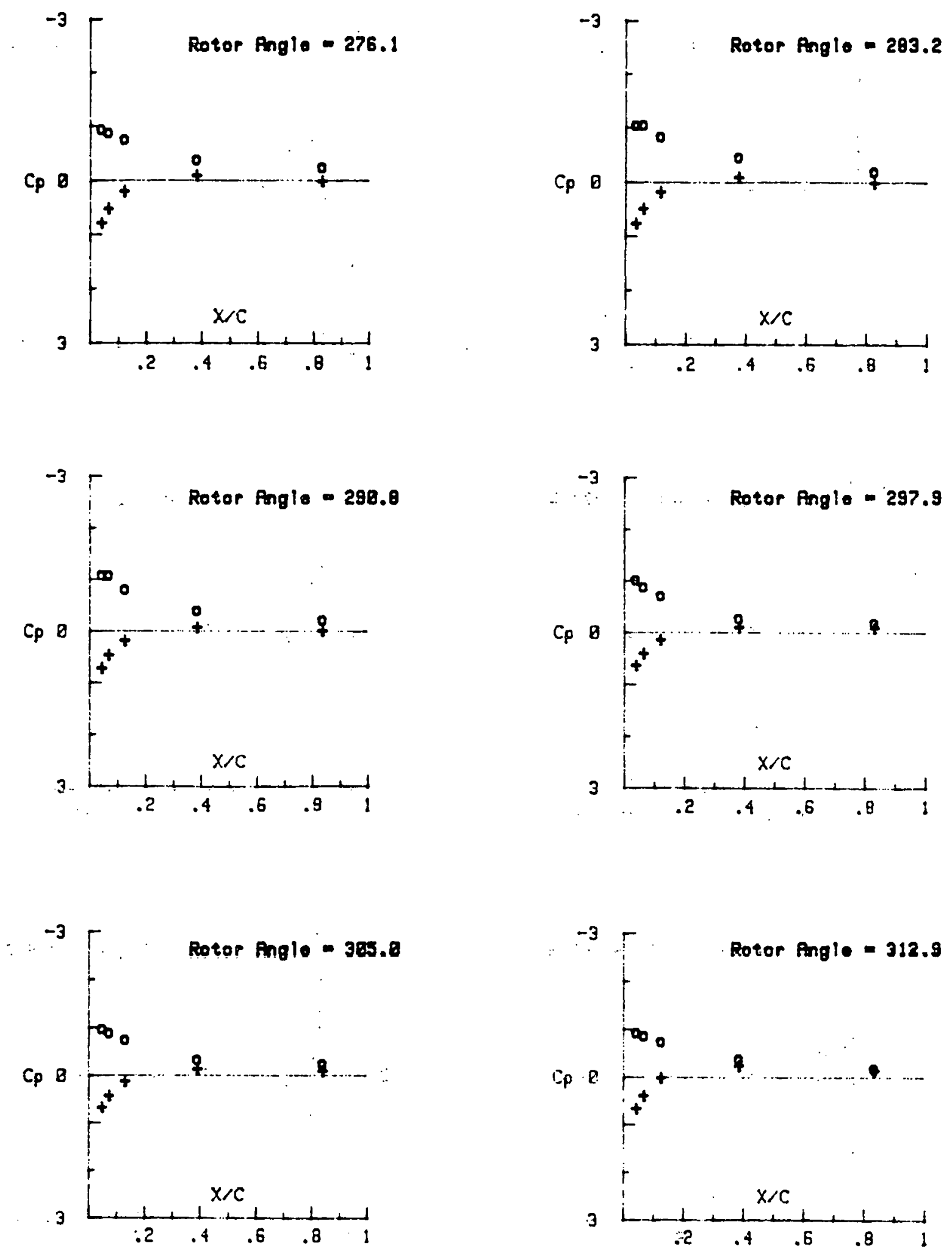

Figure E.2: Continued 

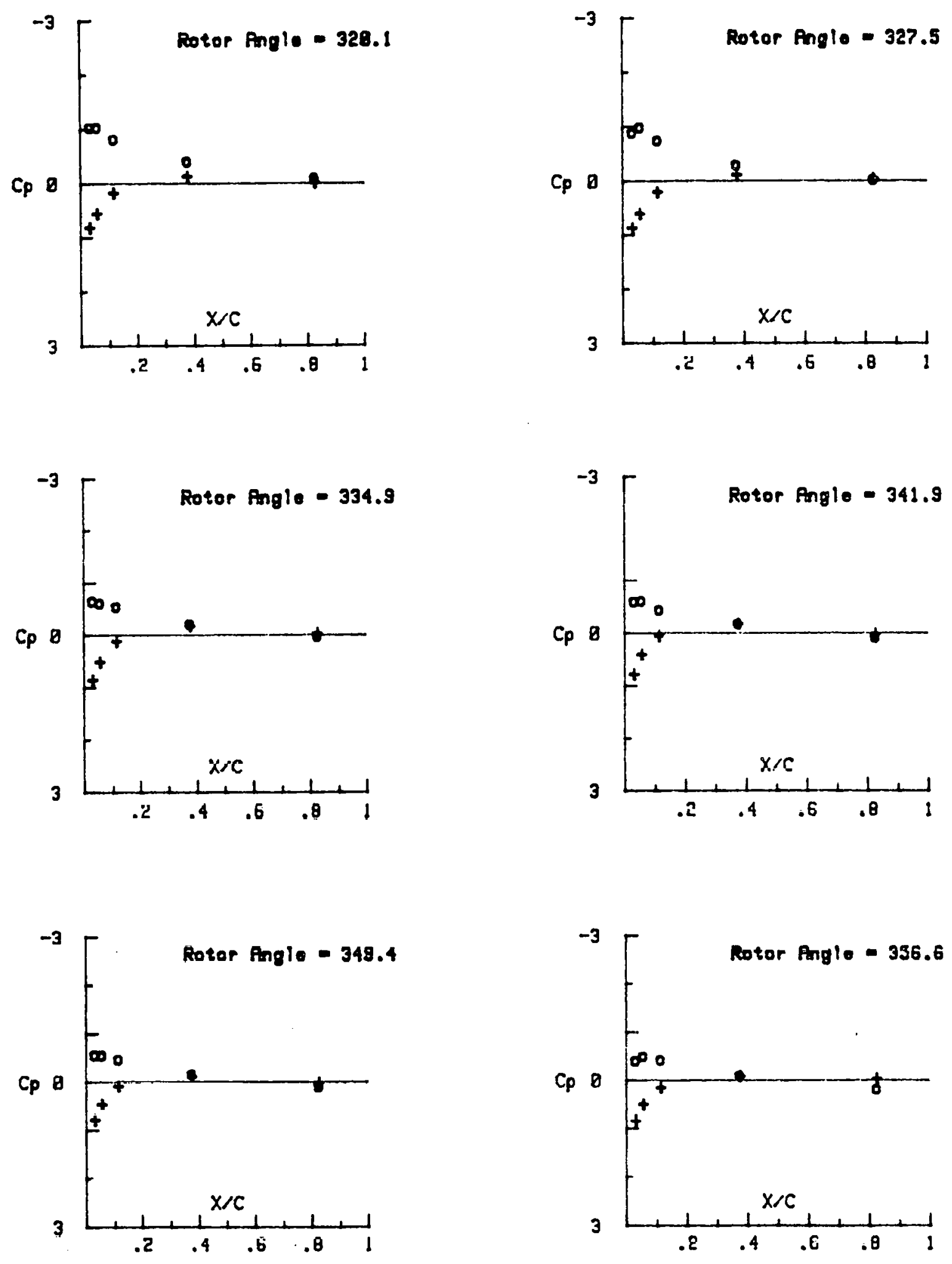

Figure E.2: Continued 

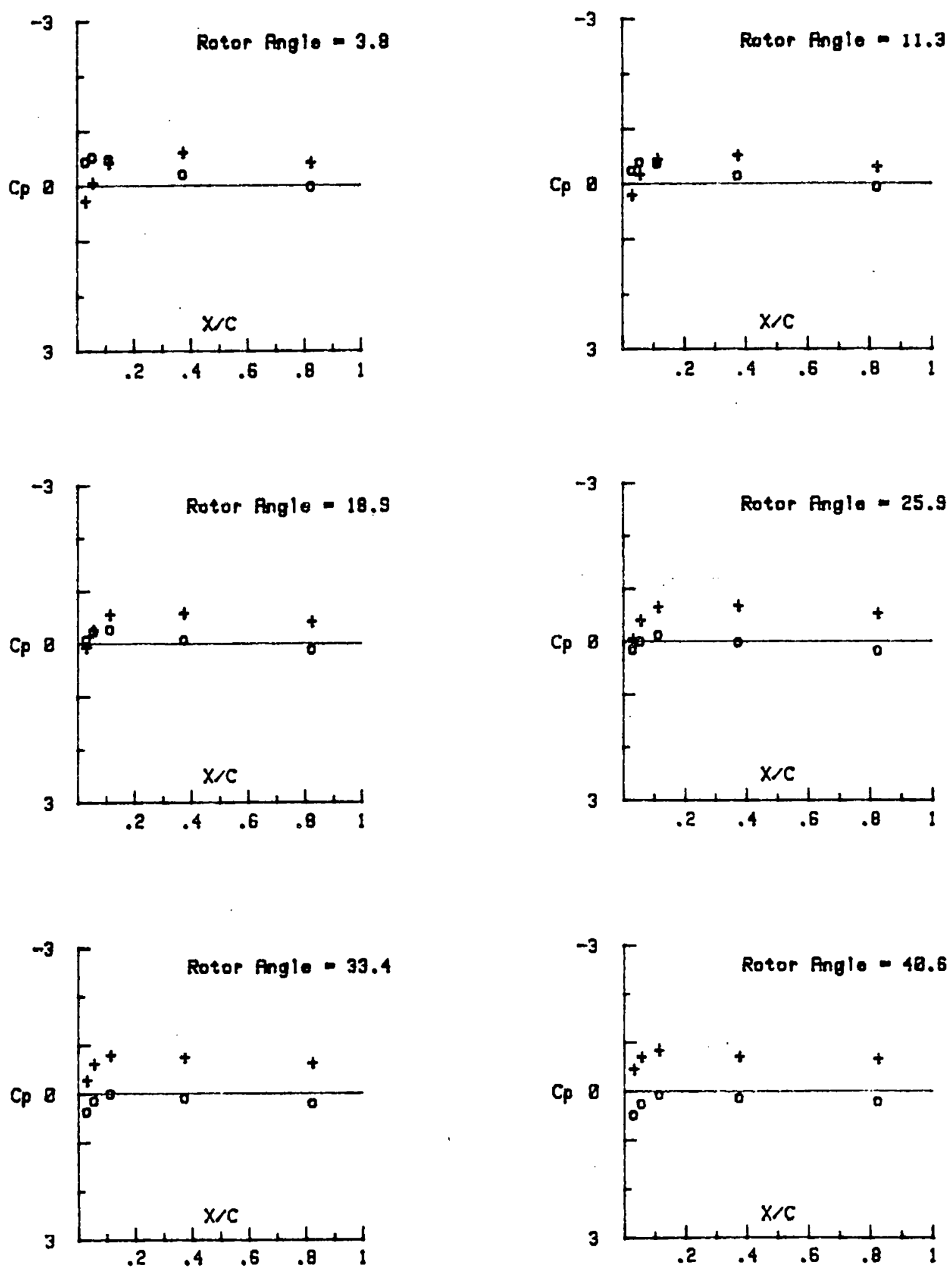

Fig. E.3: Effect of Rotor Angle on C Profiles

$\left(\mathrm{TSR}=7.6, \mathrm{C} / \mathrm{R}=0.25, \mathrm{NB}^{\mathrm{P}}=1\right.$, Outer Surface 0 , Inner Surface + ) 

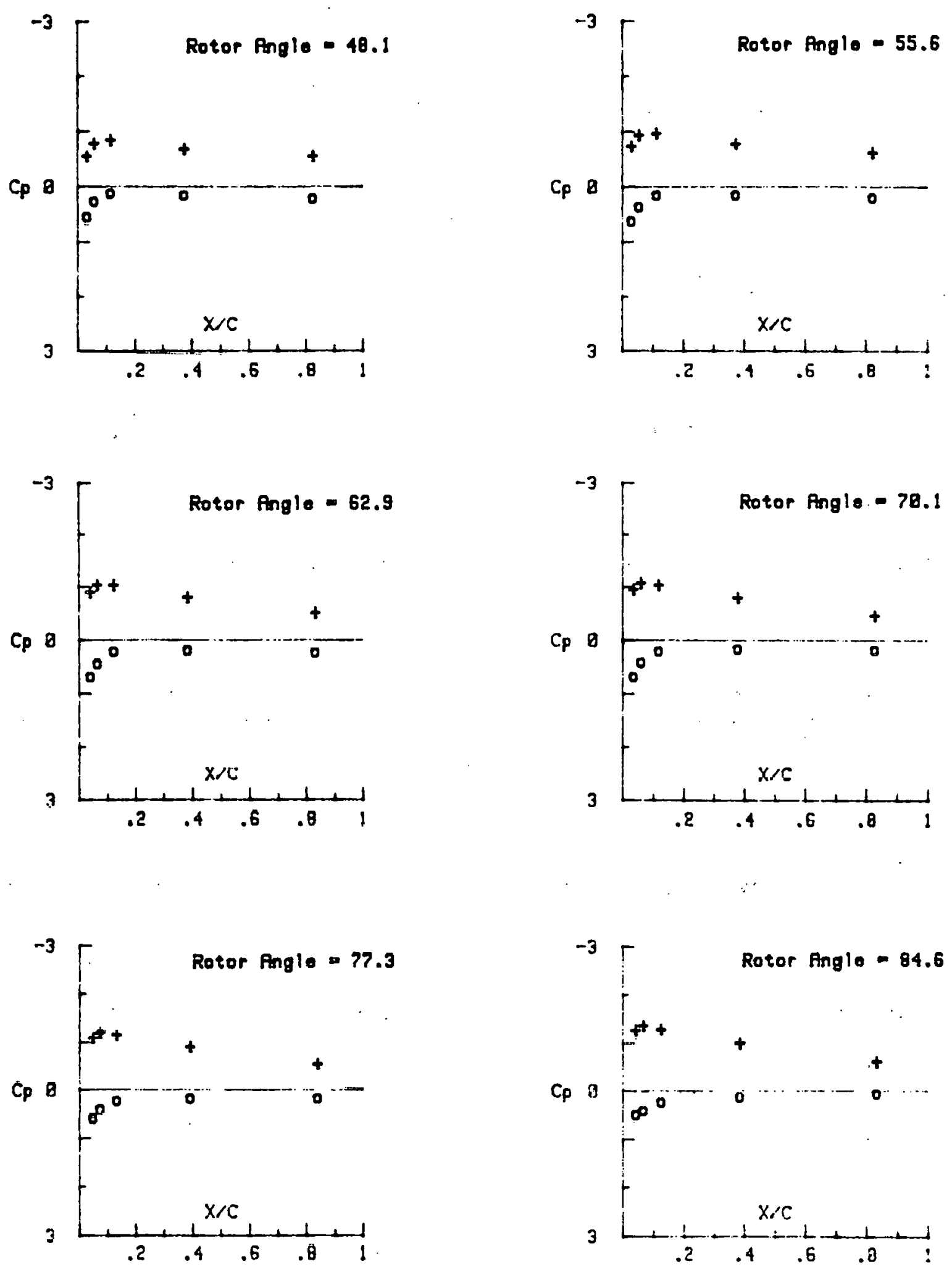

Figure E.3: Continued 

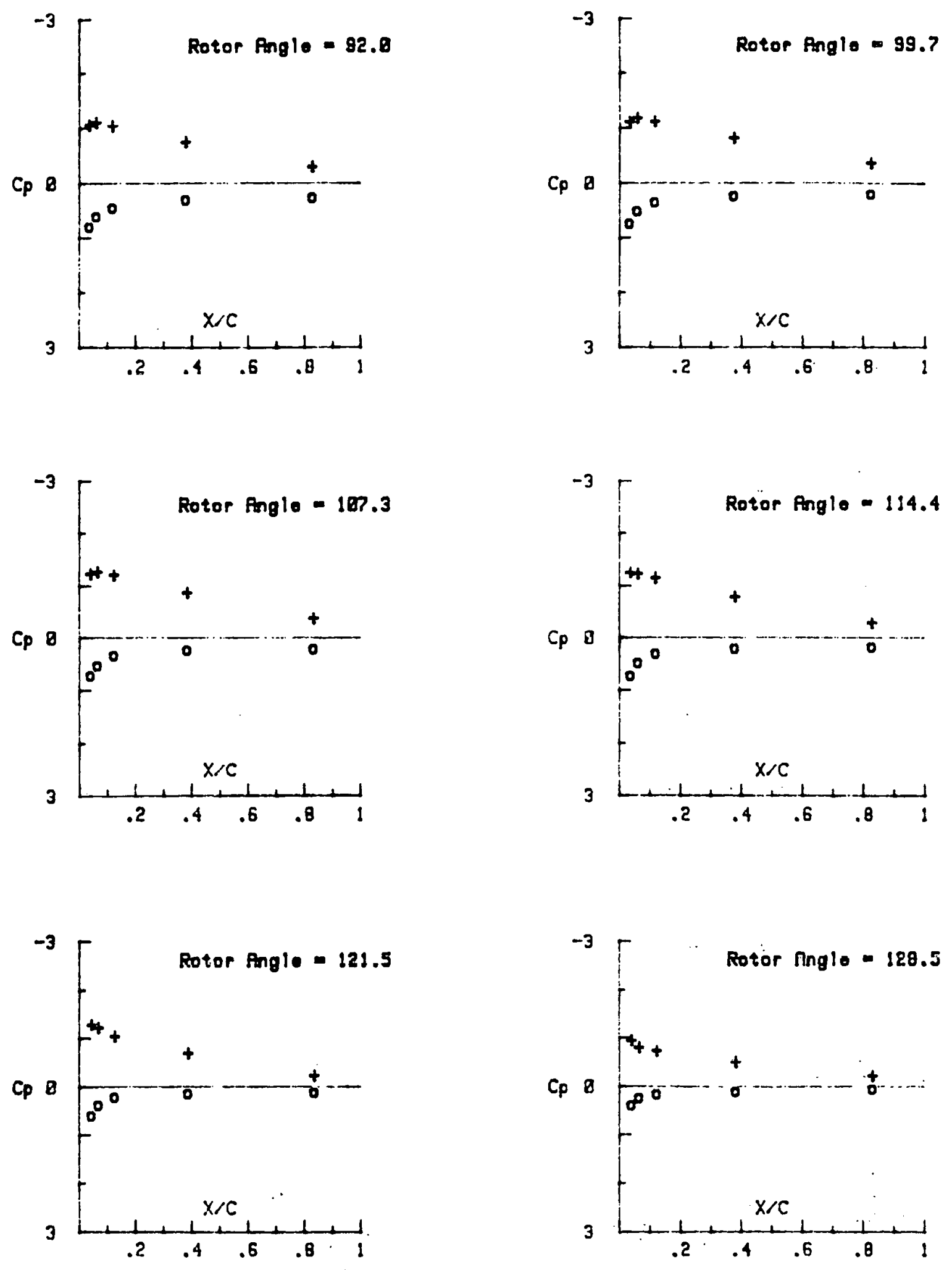

Figure E.3: Continued 

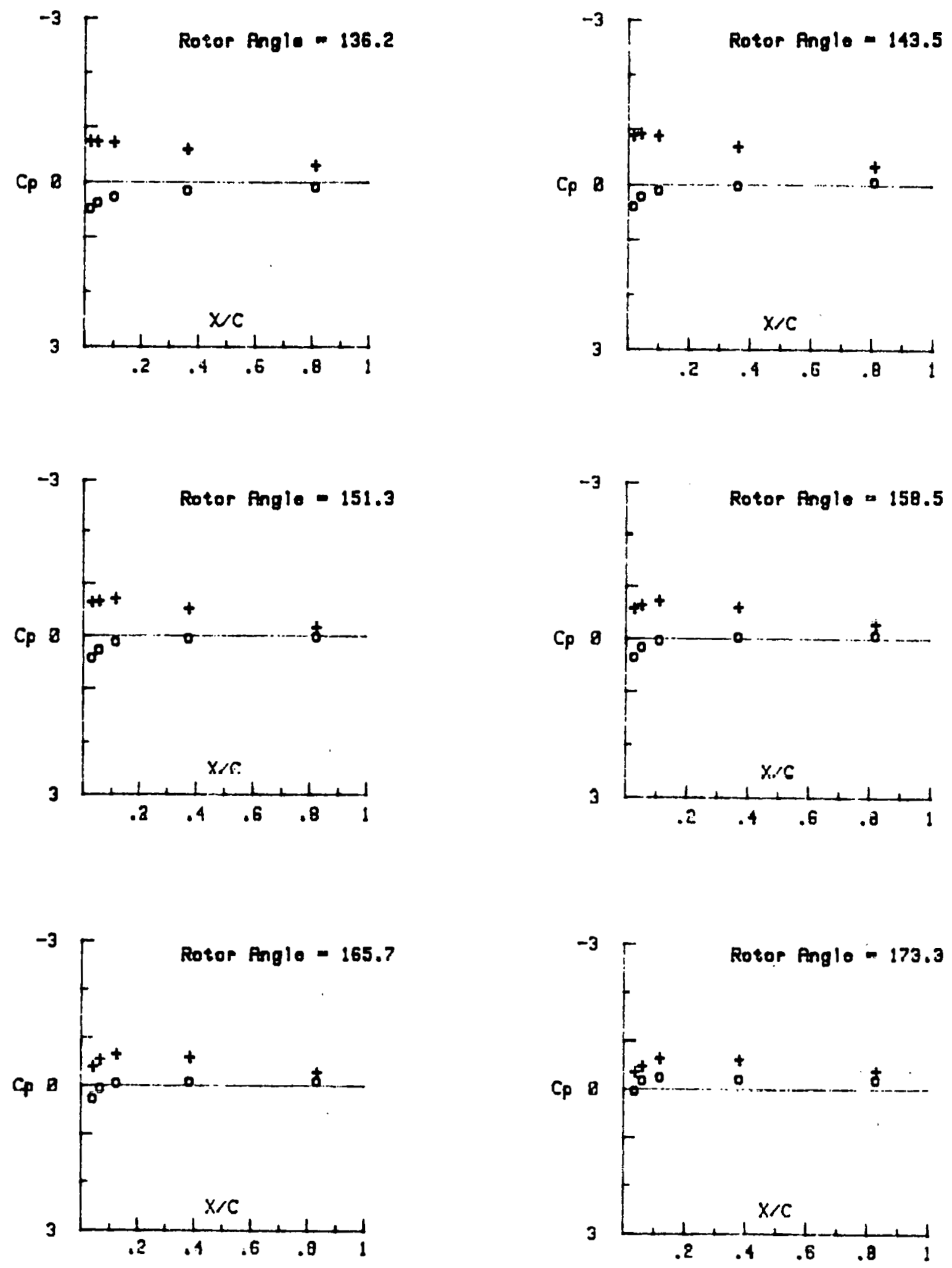

Figure E.3: Continued 

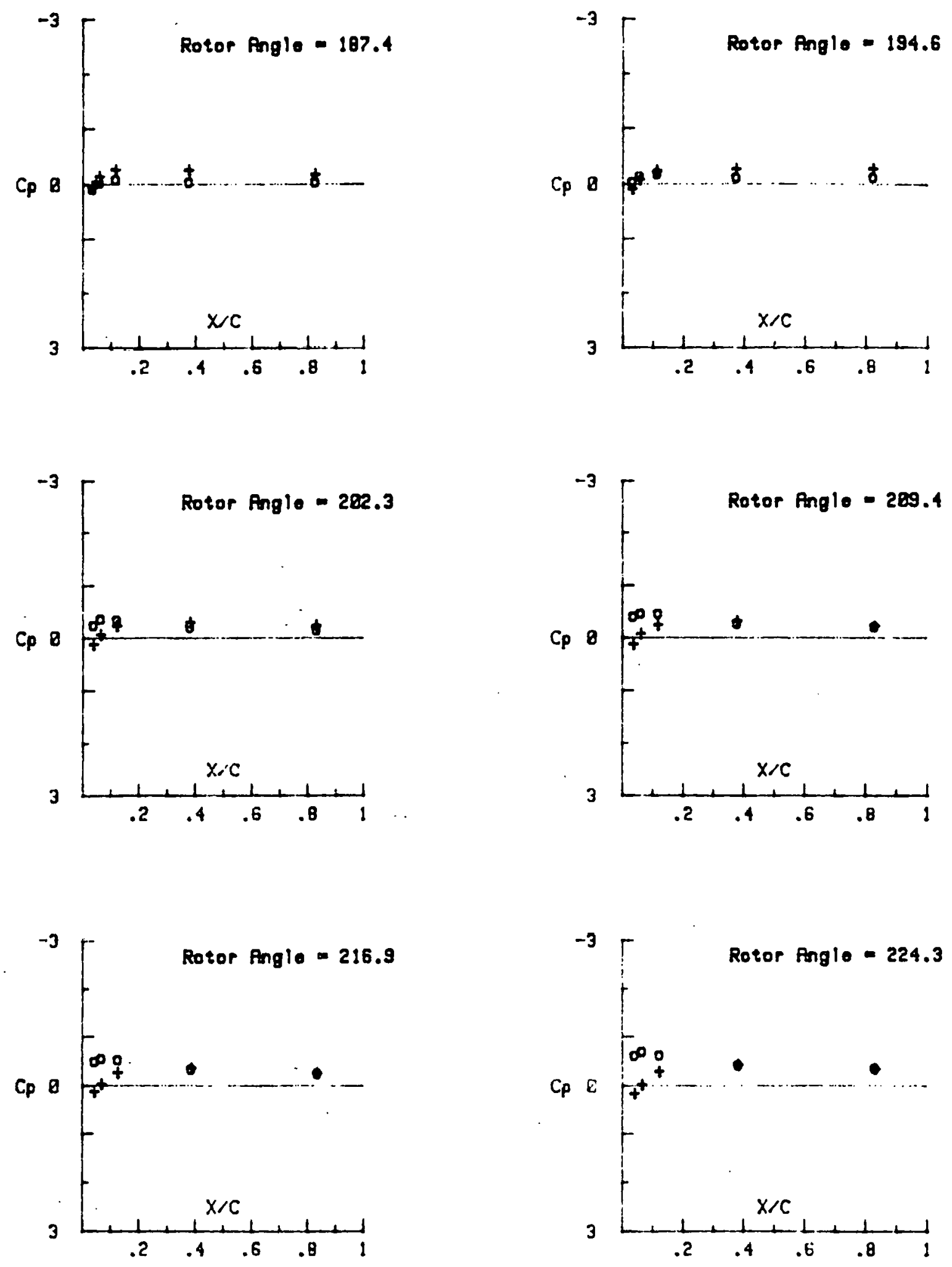

Figure E.3 : Continued 

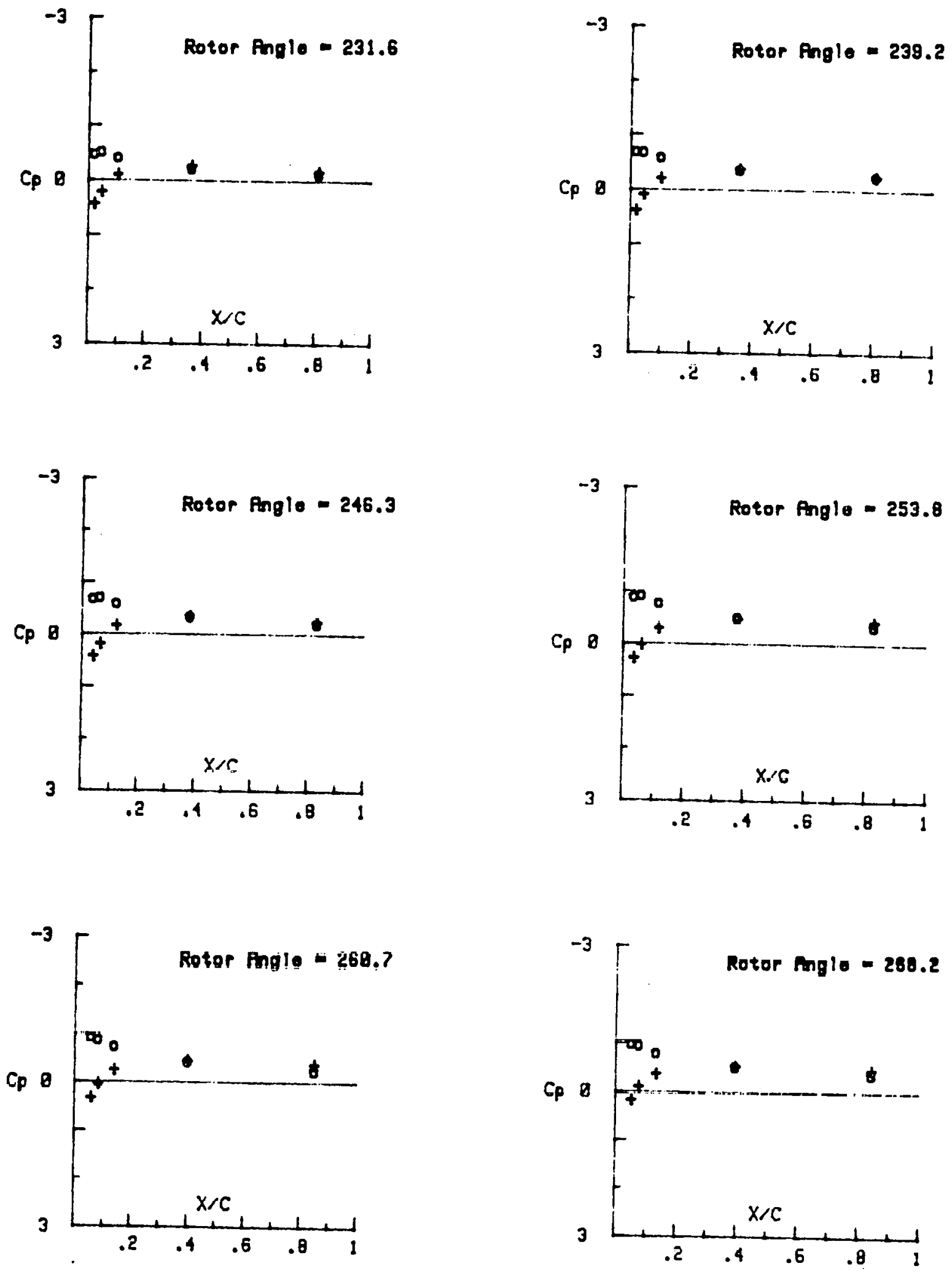

Figure E.3: Continued 

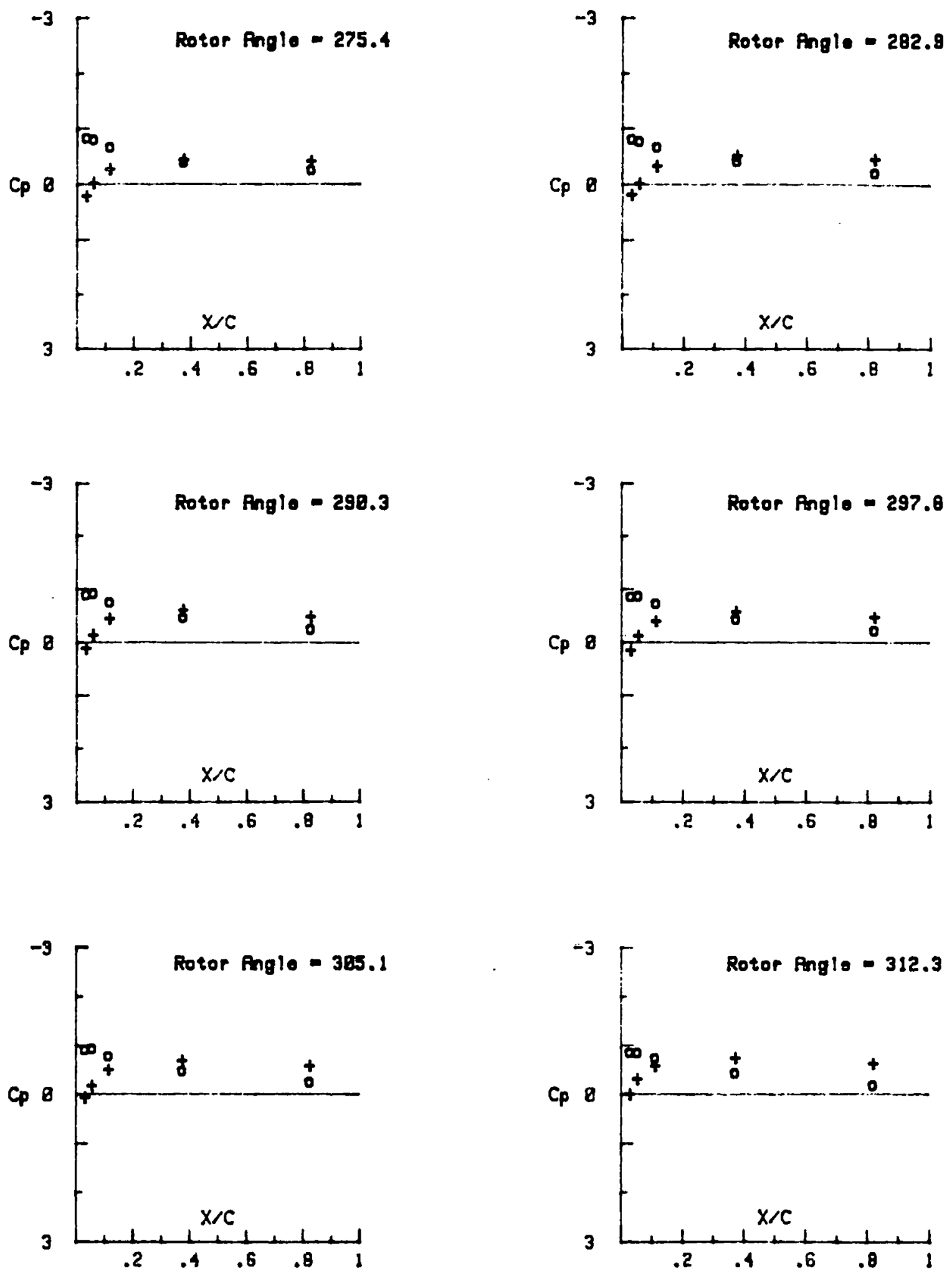

Figure E.3: Continued 

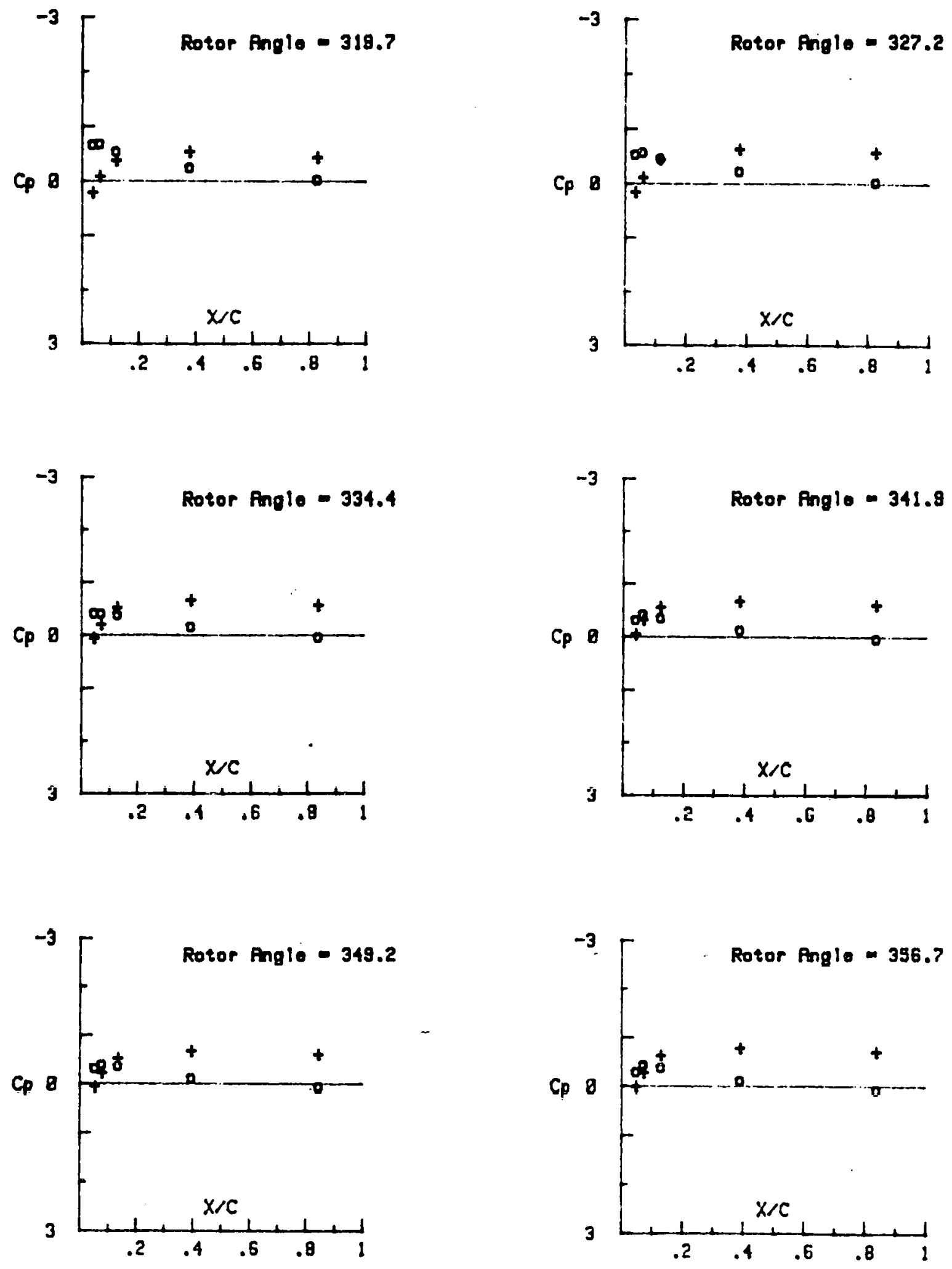

Figure E.3: Continued 


\section{DISTRIBUTION :}

Aero Engineering Department (2)

Wichita State University

Wichita, KS 67208

Attn: M. Snyder

W. Wentz

Alcoa Laboratories (4)

Alcoa Technical Center

Aluminum Company of America

Alcoa Center, PA 15069

Attn: D. K. Ai

J. T. Huang

J. R. Jombock

J. L. Prohaska

American Wind Energy Association

1609 Connecticut Avenue NW

washington, DC 20009

E. E. Anderson

South Dakota School of Mines and Technology

Department of Mechanical Engineering

Rapid City, SD 57701

Holt Ashley

Stanford University

Department of Aeronautics and

Astronautics Mechanical Engineering

stanford, CA 94305

Kevin Austin

Consolidated Edison Company of New York, Inc.

4 Irving Place

New York, NY 10003

Dr. P. J. Baum

Institute of Geophysics

and Planetary Physics

University of California

Riverside, CA 92521

F. K. Bechtel

Washington State University

Department of Electrical Engineering

College of Engineering

Pullman, WA 99163 
M. E. Beecher

Arizona State University

University Library

Tempe, AZ 85281

K. Bergey

University of Oklahoma

Aero Engineering Department

Norman, OK 73069

R. R. Berky

Alco Energy, Inc.

8002 Lee Blvd.

Leawood, KS 66208

Steve Blake

Wind Energy Systems

Route 1, Box 93-n

Uskaloosa, kS 66066

N. Ristler

Bonneville Power Administration

P.O. Box 3621

Portland, OR 97225

R. Camerero

Faculty of Applied Science

University of Sherbrooke

Sherbrooke, Quebec

CANADA JIK 2RI

Professor V. A. L. Chasteau

Schoul of Engineering

University of Auckland

Private Bag

Auckland, NEW ZEALAND

Dr. R. H. Clark

USDA, Agricultural Research Service

Southwest Great Plains Research Center

Bushland, TX 79012

Dr. D. E. Cromock

Mechanical and Aerospace Engineering Department

University of Massachusetts

Amherst, MA 01003

Gale B. Curtis

Curtis Associates

3089 Oro Blanco Drive

Colorado Springs, CO 80917 
DOE/ALO (2)

Albuquerque, NM 87115

Attn: G. P. Tennyson

DOE Headquarters/Wind Energy Technology Division (20)

1000 Independence Avenue

washington, DC 20585

Attn: L. V. Divone

P. Goldman

C. W. Dodd

School of Engineering

Southern Illinois University

Carbondale, IL 62901

D. D. Doer $r$

Kaiser Aluminum and Chemical Sales, Inc.

6177 Sunol Blud.

P.O. Box 877

Pleasonton, CA 94566

Dominion Aluminum Fabricating, Ltd. (2)

3570 Hawkestone Road

Mississauga, Ontario

CANADA L5C 2V8

Attn: L. Schienhein

C. Wood

J. B. Dragt

Nederlands Energy Research Foundation (E.C.N.)

Physics Department

Westerduinweg 3 patten ( $n h$ )

THE NETHERLANDS

Alfred J. Eggers, Jr.

Chairman of the Board

RANN, Inc.

260 Sheridan Ave., Ste. 414

Palo Alto, CA 94306

D. W. Dunham

Commonwealth Electric Co.

Box 368

Vineyard Haven, MA 02568

F. R. Eldriage, Jr.

The Mitre Corporation

1820 Dolley Madison Blvd.

McLean, VA 22102 
Electric Power Research Institute 3412 Hillview Avenue

Pajo Alto, CA 94304

Attn: E. Demeo

F. Goodman

S. Kohan

R. G. Ferreira

The Resources Agency

Department of Water Resources

Energy Division

14169 th street

P.O. Box 388

Sacramento, CA 95802

D. R. Finley

New England Geosystems

P.O. Box 128

East Derry, NH 03041

Flowind Corporation

2141468 th Avenue South

Kent, WA 98031

Attn: S. Tremoulet

I. E. Vas

J. D. Fock, Jr.

Department of Aerospace Engineering Sciences

University of Colorado

Boulder, CO 80309

G. A. Fontana (2)

Burns \& Roe, Inc.

800 Kinderkamack Roda

Oradell, NJ 07649

Dr. L. C. Frederick

Public Service Company of New Hampshire

1000 Elm Street

Manehestcr, NH 03105

H. Gerardin

Mechanical Engineering Department

Faculty of Sciences and Engineering

Universite Laval-Quebec

CANADA GIK 7P4

E. Gilmore

Amarillo College

Amarillo, TX 79100 
R. T. Griffiths

University College of Swansea

Department of Mechanical Engineering

Singleton Park

Swansea SA2 8PP

UNITED KINGDOM

Professor G. Greqorek

Ohio State University

Aeronautical and Astronautical Department

2070 Neil Avenue

Columbus, $\mathrm{OH} \quad 43210$

A. A. Hagman

Kaiser Aluminum and Chemical Sales, Inc.

14200 Cottage Grove Avenue

Dolton, IL 60419

Professor N. D. Ham

Massachusetts Institute of Technology

77 Massachusetts Avenue

Cambridge, MA 02139

Capt. J. L. Hanson

United States Air Force

Energy Technology Liaison Office

NGD

DOE/ALO

Alhisquerque, NM 87115

C. F. Harris

Wind Engineering Corporation

Airport Industrial Area

Box 5936

Lubbock, TX 79415

W. L. Harris

Aero/Astro Department

Massachusetts Institute of. Technology

Cimbridge, MA 02139

Terry Healy (2)

Rockwell International

Rocky Flats Plant

P.O. Box 464

Golden, CO 80401

Helion, Inc.

Box 445

Brownsuille, CA 95919

Dist- 5 


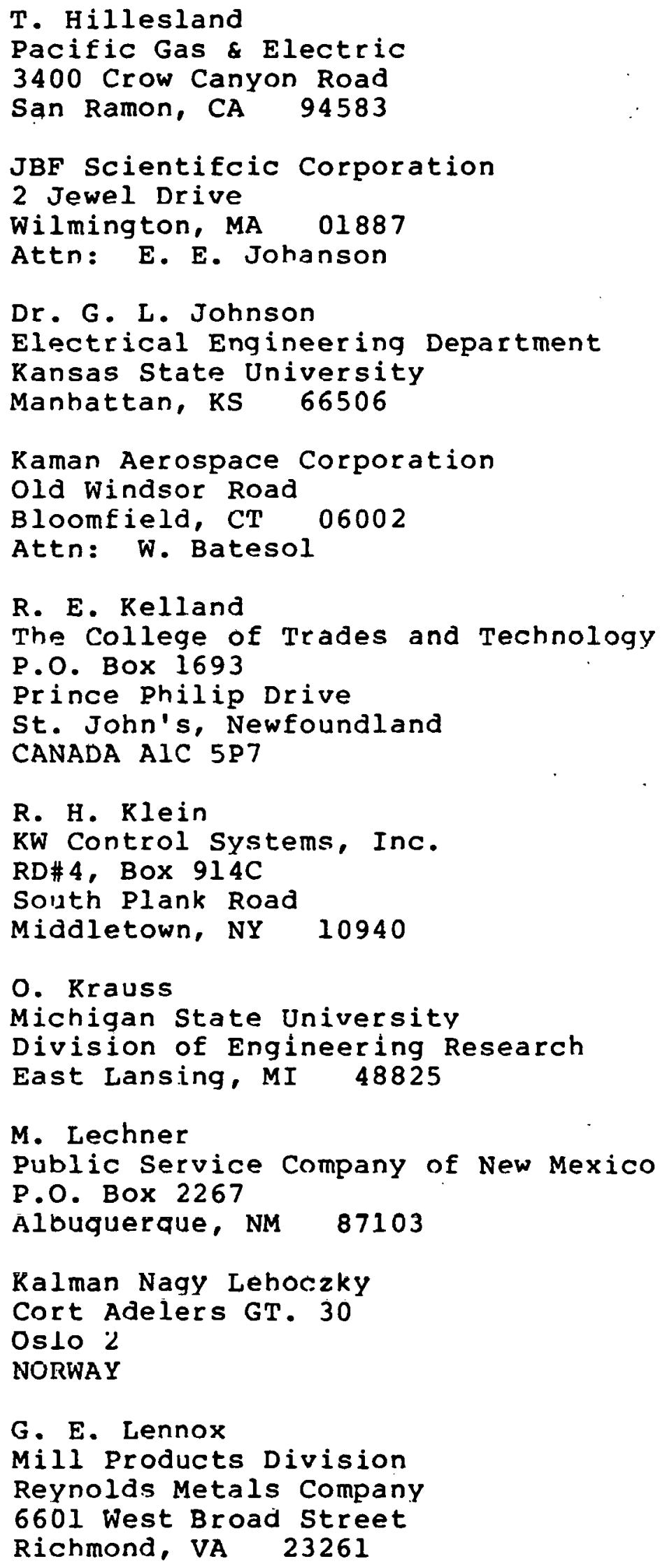


J. Lerner

State Energy Commission

Research and Development Division

1111 Howe Avenue

Sacramento, CA 95825

L. Liljidahi

Building 005, Room 304

Barc-West

Beltsville, MD 20705

P. B. S. Lissaman

Aeroenvironment, Inc.

660 South Arroyo Parkway

Pasadena, CA 91105

Olle Ljungstrom

FFA, The Aeronautical Research Institute Box 11021

S-16111 Bromma

SWEDEN

Beatrice de Saint Louvent

Establissement d'Etudes et de Recherches Metenrologigues

77. Rue de Serves

92106 Boulogne-Billancourt Cedex

FRANCE

Ernel L. Luther

PRC Energy Analysis Co.

7600 Old Springhouse Road

McLean, VA 22101

E. L. Markowski

Motorola, Inc.

G.E.D.

Mail Drop 1429

8201 E. McDowell Road

P.O. Box 1417

Scottsdale, AZ 85252

Frank Matanzo

Dardalen Associates

15110 Frederick Road

Woodbine, MD 21797 
H. S. Matsuda

Composite Materials Laboratory

Pioneering R\&D Laboratories

Toray Industries, Inc.

Sonoyama, Otsu, Shiga

JAPAN 520

J. R. McConnell

Tumac Industries, Inc.

650 Ford street

Colorado Springs, co 80915

R. N. Meroney

Colorado State University

Department of Civil Engineering

Fort Collins, Co 80521

G. N. Monzion

Department of ticonomic planning and Development

Barrett Building

Cheyenne, WY $\mathbf{8 2 0 0 2}$

Napier College of Commerce and Technology

Tutor Librarian, Technology Faculty

Colinton Road

Edinburgh, EHIO 5DT

ENGLAND

NASA Lewis Research Center (4)

21000 Brookpark Road

Cleveland, OII $\mathbf{4 4 1 3 5}$

Attn: D. Baldwin

J. Savino

A. A. Nedd

The Power Company, Inc.

P.O. Box 221

Genesee Depot, WI 53217

V. Nelson

West Texas State University

Department of Physics

P.O. Box 248

Canyon, TX 79016

Leander Nichols

Natural Power, Inc.

New Boston, NH 03070 
Roger O'Hara

Energy Times

909 NE $43 \mathrm{rd}$

Suite 308

Seattle, WA 98105

Oklahoma state University

Stillwater, OK 76074

Attn: D. K. McLaughlin

ME Department

Oregon State University

Corvallis, OR 97331

Attn: R. W. Thresher

ME Department

R. E. Wilson

ME Department

Alcir de Faro Orlando

Pontificia Universidade Catolica-PUC/Rj

Mechanical Engineering Department

R. Marques de S. Vicente 225

Rio de Janeiro, BRAZIL

Ion Paraschivoiu

IREQ

1800 montee ste-Julie

Varennes, Quebec

CANADA JOL $2 \mathrm{PO}$

G. D. Park

Gates Learjet

Mid-Continent Airport

P.O. Box 7707

Wichita, KS 67277

R. A. Parmalee

Northwestern University

Department of Civil Engineering

Evanston, IL 60201

Troels Friis pedersen

Riso National Laboratory

Postbox 49

DK-4000 Roskilde

DENMARK

Helge Petersen

Riso National Laboratory

DK-4000 Roskilde

DENMARK 
Wilson Prichett, III

National Rural Electric Cooperative Association

1800 Massachusetts Avenue NW

Washington, DC 20036

R. R. Rasmussen

Utah Power and Light Co.

51 East Main Street

P.O. Box 277

American Fork, UT 84003

T. W. Reddoch

Department of Electrical Engineering

The University of Tennessee

Knoxville, TN 37916

R. G. Richards

Atlantic wind Test site

P.O. Box 189

Tignish P.E.I.

CANADA COB 2BO

A. Robb

Memorial University of Newfoundland

Faculty of Engineering and Applied Sciences

St. John's Newfoundland

CANADA AIC 557

J. R. Rodriguez

Solarwind Energy Corporation

1163 Pomona Road

Unit $A$

Corona, CA 91720

Dr. - Tng. Hans Ruscheweyh

Institut fur Leichbau

Technische Hochschule Aachen

wullnerstrasse 7

GERMANY

R. K. St. Auhin

Aerolite, Inc.

550 Russells Mille Road

South Dartmouth, MA 02748

R. L. Soheffler

Southern California Edison

$R \& D$ Department

Room 497

P.O. BoX 800

Rosemead, CA 91770 
Gwen Schreiner

Librarian

National Atomic Museum

Albuquerque, NM 87185

Arnan Seginer

Professor of Aerodynamics

I'echnion-Israel Institute of Technology

Department of Aeronautical Engineering

Haifa, ISRAEL

Dr. Horst Selzer

Dipl.-Phys.

Wehrtechnik und Energieforschung

ERNO-Raumfahrttechnik GmbH

Hunefeldstr. 1-5

Postfach $10 \quad 5909$

2800 Bremen 1

GERMANY

H. Sevier

Rocket and Space Division

Bristol Aerospace Ltd.

P.O. Box 874

Winnipeg, Manitoba

CANADA R3C $2 S 4$

P. N. Shankar

Aerodynamics Division

National Aeronautical Laboratory

Bangalore 560017

INDIA

David Sharpe

Kingston Polytecnic

Canbury Park Road

Kingston, Surrey

UNITED KINGDOM

Kent Smith

Instituto Technologico Costa Rico

Apartado 159 Cartago

COSTA RICA

L. H. Soderholm

Iowa State University

Agricultural Engineering, Room 213

Ames, IA 50010 
G. Stacey

The University of Reading

Department of Engineering

Whiteknights, Reading, RG6 2AY

ENGLAND

Bent Sorenson

Roskilde University Center

Energy Group, Bldg. 17.2

IMFUFA

P.O. BOX 260

DK-400 Roskilde

DENMARK

D. T. Stjernholm

Morey/Stjernholm and Associates

1050 Magnolia street

Colorado Springs, cu 80907

G. W. Stricker

130 Merchant St. \#1104

Honolulu, HI 96813

C. J. Swet

Route 4

Box 358

Mt. Airy, MD 21771

John Taylor

National Research Council

ASEB

2101 Constitution Avenue

Washington, DC 20418

R. J. Templin (3)

Low Speed Aerodynamics Section

NRC-National Aeronautical Establishment

Ottawa 7, Ontario

CANADA KLA OR6

Texas Tech University (3)

P.O. Box 4389

Lubbock, TX 79409

Attn: J.W. Oler ME Department

j. Striokland

ME Department 
J. M. Turner

Terrestrial Energy Technology Program office

Energy Conversion Branch

Aerospace Power Division

Aero Propulsion Laboratory

Department of the Air Force

Air Force Wright Aeronautical Laboratories (AFSC)

Wright-Patterson Air Force Base, OH 45433

United Engineers and Constructors, Inc.

Advanced Engineering Department

30 South 17th street

Philadelphia, PA 19101

Attn: A. J. Karalis

University of New Mexico

New Mexico Engineering Research Institute

Campus P.O. Box 25

Albuquerque, NM

87131

Attn: G. G. Leigh

Jan Vacek

Eolienne experimentale

C.P. 279, Cap-aux-Meules

Iles de la Madeleine, Quebec

CANADA

P. N. Vosburgh

Forecast Industries, Inc.

3500A Indian School Road NE

Albuquerque, NM 87106

Otto de Vries

National Aerospace Lahoratory

Anthony Fokkerweg 2

Ams terdam 1017

THE NETHERLANDS

R. Walters

West Virginia University

Department of Aero Engineering

1062 Kountz Avenue

Morgantown, WV 26505

G. R. Watson

The Energy Center

Pennine House

4 Osborne Terrace

Newcastle upon Tyne NE2 INE

UNITED KINGDOM 
Tom watson

Canadian Standards Association

178 Rexdale Blvd.

Rexdale, Ontario

CANADA M9W IR3

R. G. Watts

Tulane University

Department of Mechanical Engineering

New Orleans, LA 70018

Gary Wayne

Department of Mechanical and Aerospace Engineering Upson Hall

Cornell University

Ithaca, NY 14853

Larry Wendell

Battolle-Parific Northwest Laboratory

P.O. Box 999

Richland, WA 99352

T. Wentink, Jr.

University of Alaska

Geophysical Institute

Fairbanks, AK 99701

D. Westlind

Central Lincoln People's Utility District

2129 North Coast Highway

Newport, OR 97365-1795

West Texas State University

Government Depository Lihrary

Number 613

Canyon, TX 79015

Wind Energy Report

Box 14

102 S. Village Avenue

Rockville Centre, NY 11571

Attn: Farrell smith seiler

Wind Power Digest

P. O. Box 700

Bascom, OH 44809

Attn: Michael Evans

Wind Program Manager

Wisconsin Division of State Energy

8th Floor

101 South Webster street

Madison, WI 53702 


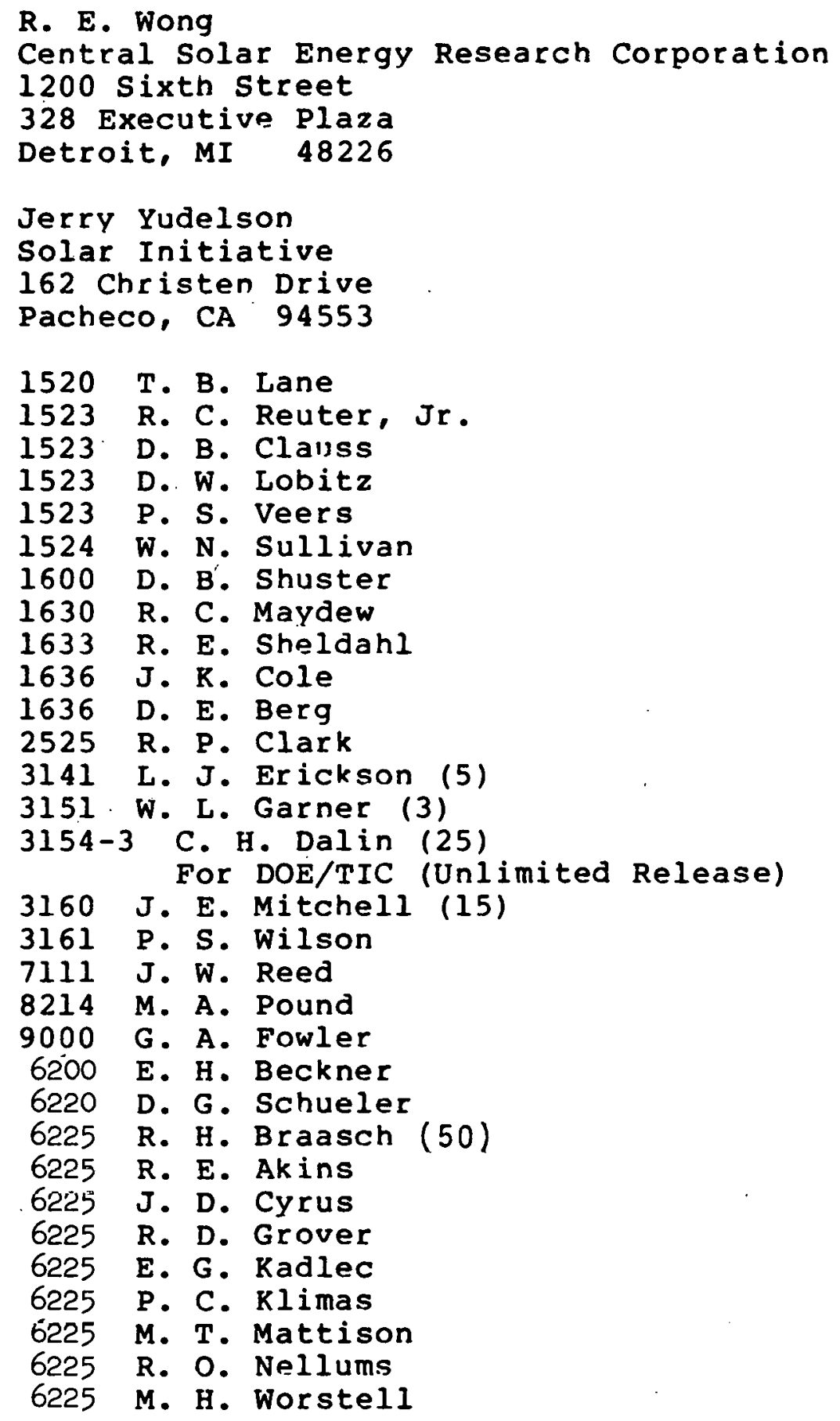

